Zweiter Beratungsgegenstand:

\title{
Soziale Gleichheit: Voraussetzung oder Aufgabe der Verfassung?
}

1. Bericht von Professorin Dr. Ulrike Davy, Bielefeld

Die Menschen werden kleiner sein, wenn wir gegangen sind. Theodor Kramer, 1943

Inhalt

Seite

I. Einleitung . . . . . . . . . . . . . . . . . . 124

II. Warum Gleichheit? . . . . . . . . . . . . . . 124

III. Grundgesetz und soziale Gleichheit . . . . . . . . . . . 130

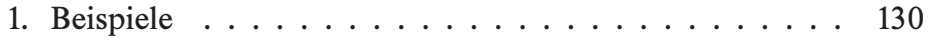

2. Gesundheit und Ungleichheit . . . . . . . . . . . 137

a) Gleichheitsrecht . . . . . . . . . . 137

b) Menschenwürde .................. . . . . . 140

3. Alterssicherung und Ungleichheit .......... . . . 145

4. Grundsicherung und Ungleichheit . . . . . . . . . . 147

a) Streit um Bedarfe . . . . . . . . . . . . . 147

b) Multiple Exklusion . . . . . . . . . . . . . . . 149

IV. Europäisierung der Gleichheit . . . . . . . . . . . 151

1. Angleichung der Lebens- und Arbeitsbedingungen . . . 151

2. Unionsbürgerschaft: Recht auf soziale Teilhabe . . . . . 153

3. Mehr soziale Gleichheit . . . . . . . . . . . . 155

V. Globale soziale Ungleichheit: Verantwortung für die Welt . . 158

1. Neue Semantiken . . . . . . . . . . . . . 158

Ich danke Patrick Blömeke, Martin Diewald, Bettina Heintz, Hartmut Krauß, Lutz Leisering, John Veit-Wilson, Ulrich Wenner, Ewald Wiederin und Hans F. Zacher für wichtige Hinweise und willkommene Ermunterung. Benjamin Davy danke ich für viel mehr als das (תודה). Daniela Krause hat mir mit viel Geduld die Welt der SOEP-Daten erschlossen. Mein Dank für mühevolle redaktionelle Hilfe geht an Hacer Bolat, Bernadette Goesmann, Annika Halbmeier, Tina Hapke, Christiane Hastaedt und Charlotte Strauch. 
2. Neue Rechtsnormen? . . . . . . . . . . . . . . . . 163

3. Pflicht zur Beseitigung globaler sozialer Ungleichheit . . 168

VI. Zusammenfassung . . . . . . . . . . . . . . . . 170 


\section{Einleitung}

Die Umsetzung der Agenda 2010 - sie steht für tiefe Einschnitte in die soziale Sicherheit - ist von einem nachhaltigen Diskurs über soziale Gerechtigkeit begleitet. Der Diskurs beschäftigt die Medien, die Politik und die Wissenschaft, allen voran die Soziologie, die darüber nachdenkt, wie die Neuausrichtung der Sozialstaatlichkeit zu deuten ist. ${ }^{1}$ Jedenfalls in den Medien und in der Politik ist soziale Ungleichheit ein wiederkehrender Bezugspunkt.2 Die Rede ist von Spaltungslinien in der Gesellschaft und sozialen Verwerfungen. Politisch brisant ist der Diskurs, weil parallel dazu eine politische Partei entstanden ist, die über beträchtliches Stimmenpotential verfügt. Die Linkspartei sieht ihre Hauptaufgabe darin, Gleichheit mit Freiheit zu verbinden. ${ }^{3}$

Die angesprochenen Phänomene bleiben so vage, dass ich mit einer allgemeinen Frage beginne: Was ist soziale Gleichheit, weshalb ist sie bedeutsam und wo könnten rechtliche Überlegungen ansetzen?

\section{Warum Gleichheit?}

Die klarsten Antworten auf die Frage nach der Bedeutung von Gleichheit gibt die politische Philosophie. Egalitarians begründen die Relevanz der Gleichheitsidee mit einem Hinweis auf den Konsens der Menschen in der original position oder der Schiffbrüchigen auf einer unbewohnten Insel: In dieser Situation würde Gleichheit grundsätzlich zum Maßstab für die Verteilung von Ressourcen gemacht werden. ${ }^{4}$

\footnotetext{
1 Vgl. die Beiträge in: St. Leibfried/U. Wagschal (Hrsg.) Der deutsche Sozialstaat. Bilanzen - Reformen - Perspektiven, 2000; D. Döring Die Zukunft der Alterssicherung, 2002; D. Döring Sozialstaat, 2004; aus internationaler Perspektive: P. Pierson (Hrsg.) The New Politics of the Welfare State, 2001; G. Esping-Andersen/D. Gallie/J. Myles Why we need a New Welfare State, 2002.

2 Vgl. z.B. H. Prantl Das letzte Gefecht der Volksparteien, 28. 6. 2008, www.sueddeutsche.de; Kleine Anfrage der Fraktion DIE LINKE v. 9. 8. 2006, BT-Drs. 16/2388; Antrag der Fraktion Bündnis 90/Die Grünen v. 14. 11. 2007, BT-Drs. 16/7136; Antrag der Fraktion DIE LINKE v. 12. 12. 2007, BT-Drs. 16/7471.

3 Programmatische Eckpunkte v. 24. und 25. 3. 2007, unter: www.die-linke.de/ partei/dokumente.

${ }^{4}$ Die Rawlsianische Gerechtigkeitstheorie ist konzipiert für eine demokratische $\mathrm{Ge}$ sellschaft (democratic society). In einer demokratischen Gesellschaft betrachten Bürger sich als frei und gleich ( . Rawls Justice as Fairness. A Restatement, 2001, § 2.3: ,as free and equal persons"; vgl. auch J. Rawls A Theory of Justice, 1971, § 77). An diesem Ausgangspunkt hat Gleichheit keinen Bezug zu Ressourcen. Gleichheit bezieht sich vielmehr auf die Gleichheit von Menschen als Menschen (as persons): Den Verhandlungen
} 
Libertarians dagegen beziehen Gleichheit auf ein bestimmtes - eben gleiches - Bündel an Freiheitsrechten. ${ }^{5}$ Die ungleiche Verteilung von Ressourcen interessiert nicht: Ungleichheiten seien nichts weiter als das Ergebnis freier Einzelentscheidungen. ${ }^{6}$

Freilich behaupten selbst egalitarians nicht, dass Ungleichheiten stets ungerecht seien. Ungleichheiten sind bloß rechtfertigungsbedürftig: Unproblematisch scheint Ungleichheit dann, wenn die Unterschiede auf unterschiedliche Neigungen oder unterschiedlichen Einsatz zurückzuführen sind. ${ }^{7}$ Dasselbe gilt, wenn Menschen ihre Talente vergeuden, ${ }^{8}$ unterschiedlich risikobereit oder unterschiedlich vom „Glück“ gesegnet sind. ${ }^{9}$ Denn persönliche Verdienste sollten belohnt werden, Anreizsysteme seien notwendig. Schwierigkeiten bereiten Unterschiede in der Ressourcenverteilung, die auf unterschiedliche Begabungen zurückzuführen sind, auf die Geburt in eine soziale Schicht oder an einem be-

in der original position liegt die Annahme zugrunde, daß Menschen über ein gleiches $\mathrm{Maß}$ an jenen moralischen Fähigkeiten (moral powers) verfügen, die sie zur Zusammenarbeit in einer demokratischen Gesellschaft benötigen. Freie und gleiche Menschen nun würden sich - so nimmt Rawls an - in den Verhandlungen in der original position mit Blick auf ,primary goods“ (Freiheiten, Chancen, Einkommen und Vermögen, Grundlagen für die Selbstachtung) auf ein Verteilungsprinzip verständigen, das prinzipielle Gleichverteilung ins Auge faßt (Rawls Theory, § 11, 62). Ausgangspunkt der Dworkinschen Gerechtigkeitstheorie ist ein Schiffbruch ( $R$. Dworkin What is Equality? Part 2: Equality of Resources, Philosophy and Public Affairs 10/4 [1981] 283): Mehrere Schiffbrüchige gelangen auf eine unbewohnte, aber reichlich mit Ressourcen ausgestattete Insel. Das Ergebnis ihrer Verhandlungen ist nach Dworkin's Meinung: „These immigrants accept the principle that no one is antecedently entitled to any of these resources, but that they shall instead be divided equally among them" (ebd. 285).

${ }^{5}$ So zu Recht der Hinweis bei $A$. Sen Inequality Reexamined, 1992 (reprint 1995) 13. Die libertäre Position wird v.a. repräsentiert durch R. Nozick Anarchy, State, and Utopia, 1974 (reprint 1992).

${ }^{6}$ R. Nozick (Fn. 5) 149 f: „In a free society, diverse persons control different resources, and new holdings arise out of the voluntary exchanges and actions of persons“. Diese Verteilung sei auch gerecht, solange sie das Ergebnis bestimmter Regeln über den Erwerb sei. Dann nämlich seien die Individuen dazu berechtigt, die Dinge zu besitzen, die sie besitzen (entitlement theory, ebd. $150 \mathrm{ff}$.). Welche Regeln genau ,justice in acquisition“ und ,justice in transfer“ verbürgen, legt Nozick allerdings nicht offen (ebd. 153: „I shall not attempt that task here").

7 J. Rawls Restatement (Fn. 4) § 21.1; R. Dworkin (Fn. 4) 292 f, 304; A. Sen Inequality (Fn. 5) 85.

8 J. Rawls Restatement (Fn. 4) § 18.3 a.E.; A. Sen Inequality (Fn. 5) 148; N. Daniels Equality of What: Welfare, Resources, or Capabilities? Philosophy and Phenomenological Research, 50, Suppl. (1990) 273 (285).

${ }^{9}$ R. Dworkin (Fn. 4) 293 ff.; A. Sen Justice: Means versus Freedom, Philosophy and Public Affairs 19/2 (1990) 111 (116). 
stimmten Ort der Welt. ${ }^{10}$ Diese Ungleichheiten gelten als ungerecht und ausgleichspflichtig. ${ }^{11}$

Der Soziologie verdanken wir eine Schärfung des Begriffs der sozialen Ungleichheit. Soziale Ungleichheit bezeichnet eine gesellschaftlich bestimmte Lage, in der Menschen unterschiedliche Verfügungsgewalt über Ressourcen und Lebenschancen haben. ${ }^{12}$ Soziale Ungleichheit hat nicht von vornherein den Beigeschmack von Ungerechtigkeit. Die Ungleichheitsforschung will Ungleichheit in erster Linie beschreiben.13 Für die allgemeine Soziologie ist soziale Ungleichheit im Wesentlichen ein Merkmal (auch) der Moderne. ${ }^{14}$ Simmel erklärt sie mit einem Wesens-

10 J. Rawls Restatement (Fn. 4) § 16.1, § 21.1; R. Dworkin (Fn. 4) 306 ff; A. Sen Inequality (Fn. 5) 79 ff; Th. Nagel Equality and Partiality, 1991, 10ff.; Th. Pogge World Poverty and Human Rights, 2002.

11 Für Rawls müssen Ungleichheiten zugleich den Schlechtestgestellten nützen (Theory [Fn. 4]) § 11, § 13; Restatement [Fn. 4] § 13), für Dworkin dürfen die individuell verfügbaren Ressourcen eine Mindestschwelle nicht unterschreiten (Equality [Fn. 4] $312 \mathrm{ff}$.). Kritisch gegenüber Ansätzen, die ,equality of fortune“ in den Mittelpunkt rücken, E. S. Anderson What is the Point of Equality? Ethics 109/2 (1999) 287.

12 St. Hradil Soziale Ungleichheit in Deutschland, 8. Aufl. 2001 (Nachdruck 2005) 29: „In der soziologischen Terminologie wird immer dann von ,sozialer Ungleichheit' gesprochen, wenn als ,wertvoll' geltende ,Güter' nicht absolut gleich verteilt sind" (Hervorhebungen im Original). Menschen, die über solche wertvollen Güter verfügen, erscheinen danach im Verhältnis zu anderen, die über solche Güter nicht verfügen, als besseroder höhergestellt. Ähnlich R. Kreckel Politische Soziologie der sozialen Ungleichheit, 3. Aufl. 2004, 17: „Soziale Ungleichheit im weiteren Sinne liegt überall dort vor, wo die Möglichkeiten des Zuganges zu allgemein verfügbaren und erstrebenswerten sozialen Gütern und/oder zu sozialen Positionen, die mit ungleichen Macht- und/oder Interaktionsmöglichkeiten ausgestattet sind, dauerhafte Einschränkungen erfahren und dadurch die Lebenschancen der betroffenen Individuen, Gruppen oder Gesellschaften beeinträchtigt bzw. begünstigt werden." Ch. Tilly hat mit Nachdruck darauf aufmerksam gemacht, daß Ungleichheit Ergebnis eines dynamischen Prozesses ist; Ungleichheit wird hergestellt und aufrecht erhalten. Näher Ch. Tilly Durable Inequality, 1998 (exploitation, opportunity hoarding, emulation, adaptation) sowie Ch. Tilly Democracy, 2007, 111 (,organized differences in advantages by gender, race, nationality, ethnicity, religion, community, and similar classification systems").

13 So St. Hradil (Fn. 12) 29, der ausdrücklich festhält, daß der soziologische Begriff der „sozialen Ungleichheit“ es offen lasse, inwieweit es sich bei den bezeichneten Erscheinungen um problematische, da illegitime Ungleichheiten handele. Zum Forschungsstand s. ferner $H$. Esser Soziologie. Spezielle Grundlagen, Bd. 2: Die Konstruktion der Gesellschaft, 2000, 113 ff.; N. Burzan Soziale Ungleichheit. Eine Einführung in die zentralen Theorien, 3. Aufl. 2007; Th. Schwinn Soziale Ungleichheit, 2007.

14 Für G. Simmel Über sociale Differenzierung, in: G. Simmel Aufsätze 1887 bis 1890. Über sociale Differenzierung. Die Probleme der Geschichtsphilosophie (1892), hrsg. v.H.-J. Dahme, 1989, 109 ist soziale Ungleichheit im wesentlichen eine Folge der Herausbildung der verantwortlichen Einzelpersönlichkeit, für Parsons (Equality and Inequality in Modern Society, or Social Stratification Revisited, in: T. Parsons Social Sys- 


\section{zug der Menschen..$^{15}$ Esser hält sie für eine Universalie. ${ }^{16}$ Luhmann ord- net die Thematisierung von Ungleichheit der Entwicklung des Wohl- fahrtsstaates zu. ${ }^{17}$}

tems and the Evolution of Action Theory, 1977, 321 [326ff.]) eine Folge der gesellschaftlichen Organisation, für Luhmann und Esser eine Begleiterscheinung der funktionalen Differenzierung. Eingehend H. Esser Soziologie II (Fn. 13) 52, 123, 175, $233 \mathrm{ff} ., 244$. Luhmann selbst hat das Verhältnis von funktionaler Differenzierung und sozialer Ungleichheit zunächst offen gehalten. In einer Arbeit aus den 1980er Jahren zum Übergang von einer stratifikatorischen Gesellschaft zu einer funktional differenzierten Gesellschaft hält Luhmann lediglich fest, daß soziale Ungleichheit nicht nur ein Merkmal einer stratifikatorischen Gesellschaft sei, sondern auch ein Merkmal einer funktional differenzierten Gesellschaft, und der Klassenbegriff daher nicht obsolet werde; die ausdifferenzierte Klassengesellschaft erlaube aber größere Freiheiten bei der Rollenwahl. Näher N. Luhmann Zum Begriff der sozialen Klasse, in: N. Luhmann (Hrsg.) Soziale Differenzierung. Zur Geschichte einer Idee, 1985, $119 \mathrm{ff}$. Später beschreibt Luhmann soziale Ungleichheit über die Mechanismen von Inklusion bzw. Exklusion in Teilsysteme. Da die Teilsysteme je für sich Inklusion bzw. Exklusion regeln, können für Menschen Mehrfachabhängigkeiten entstehen, die Exklusionseffekte und Ungleichheitseffekte verstärken. Dazu N. Luhmann Inklusion und Exklusion, in: N. Luhmann Soziologische Aufklärung 6. Die Soziologie und der Mensch, 1995, $237 \mathrm{ff}$; N N Luhmann Die Gesellschaft der Gesellschaft, Bd. 2, 1997, 618ff.; N. Luhmann Die Politik der Gesellschaft, hrsg. v. A. Kieserling, 2000, 427. Weiterführend dann Stichweh in: R. Stichweh Inklusion und Exklusion. Studien zur Gesellschaftstheorie, 2005. Zum Verhältnis von Differenzierungstheorie und sozialer Ungleichheit vgl. insb. die Beiträge von Th. Schwinn, L. Leisering und R. Stichweh in: Th. Schwinn (Hrsg.) Differenzierung und soziale Ungleichheit, 2004, 9, 238, 353.

15 „Tausendfache Erfahrung“ zeige, daß „Gleichmachung“ den Wunsch der Menschen, andere zu überflügeln, nicht beseitigen könne. So G. Simmel Über sociale Differenzierung, in: G. Simmel Aufsätze 1887 bis 1890. Über sociale Differenzierung. Die Probleme der Geschichtsphilosophie (1892), hrsg. v.H.-J. Dahme, 1989, 109 (233). Skeptisch gegenüber Gleichheitssemantiken auch G. Simmel Rosen. Eine soziale Hypothese, in: G. Simmel Individualismus der modernen Zeit und andere soziologische Abhandlungen, hrsg. v. O. Rammstedt, 2008, 355.

16 H. Esser Soziologie II (Fn. 13) 214: „Es gibt keine bekannte Gesellschaft, die nicht ... mehr oder weniger deutliche Unterschiede in der vertikalen Rangordnung der Akteure kennen würde, die bestimmte Eigenschaften haben und bestimmte Arten von Ressourcen kontrollieren“. Esser spricht sozialer Ungleichheit freilich eine „Doppelnatur“ zu: Ungleichheit beruhe zum einen auf Unterdrückung und Ausbeutung, sei aber zum anderen eine wichtige Bedingung für den materiellen Wohlstand der Menschen (ebd. 221).

${ }_{17}$ Vgl. etwa N. Luhmann Politik (Fn. 14) 139: „Demokratie und Parteienkonsens führen dazu, daß immer mehr ,Notlagen' und immer mehr sinnvolle Wünsche entdeckt und formuliert werden, die dann in Forderungen an das politische System umgemünzt werden“. Oder, drastischer, ebd. 215: „In zunehmendem Umfange stellt der Wohlfahrtsstaat sich Probleme, die als zu lösende Probleme, also als lösbare Probleme vorgestellt werden ... Alles in allem gleicht der Wohlfahrtsstaat dem Versuch, die Kühe aufzublasen, um mehr Milch zu bekommen“. Daraus resultierten wirtschaftliche Folgen, insb. Probleme bei der Finanzierung, die das Teilsystem Wohlfahrtsstaat selbst nicht kontrol- 
Der Blick in die Nachbarwissenschaften erlaubt eine erste These: Soziale Gleichheit ist weder Voraussetzung noch Aufgabe einer Verfassung. ${ }^{18}$ Das gilt für die Verfassungsordnung des Grundgesetzes, für das Gemeinschaftsrecht und das Völkerrecht. Keine der Ordnungen beruht auf einem egalitären Verteilungsprinzip. Moderne Verfassungen haben gerade die Funktion einer Klammer für Differenzierungen und, darin eingeschlossen, soziale Ungleichheiten. ${ }^{19}$

lieren könne. Kritisch zur Eigendynamik des Wohlfahrtsstaates auch H. F. Zacher Das soziale Staatsziel, HStR II, 3. Aufl. 2004, § 28 Rn. 84: „Nach einer permanenten Erfahrung der Sozialpolitik läßt jede Lösung eines sozialen Problems eine Vielzahl neuer Probleme sichtbar werden“. Vgl. weiter H. F. Zacher Der Wandel der Arbeit und der sozialen Sicherheit im internationalen Vergleich, ZIAS 13/1 (1999) 1; H. F. Zacher Die Bundesrepublik Deutschland als Sozialstaat: eine Geschichte des sozialen Einschlusses im Zeichen von Nationalisierung und Internationalisierung, ZIAS 16/3 (2002) 193; H. F. Zacher Der soziale Bundesstaat, FS Schmitt Glaeser, 2003, 199.

${ }_{18}$ Der Begriff „Verfassungsvoraussetzungen“ faßt alle Bedingungen zusammen, die grundsätzlich außerhalb der Verfassung selbst liegen und das Funktionieren der Verfassung sicherstellen. Sie entstammen v. a. dem Bereich der Ökonomie, der Politik und der Kultur. Zum Begriff näher H. Krüger Verfassungsvoraussetzungen und Verfassungserwartungen, FS Scheuner, 1973, 285; J. Isensee Staat und Verfassung, HStR II, 3. Aufl. 2004, § 15 Rn. 169; E.-W. Böckenförde Demokratie als Verfassungsprinzip, HStR II, 3. Aufl. 2004, $§ 24$ Rn. 58 ff. Da Verfassungsvoraussetzungen - z.B. politische Bildung als Voraussetzung für die Demokratie - auch beeinflußt und hergestellt werden können, überlappt sich der Begriff der Verfassungsvoraussetzungen zum Teil mit dem Begriff der Staatsaufgaben, soweit damit - mit R. Herzog Allgemeine Staatslehre, 1971, 106 schlicht die „Bereiche moderner staatlicher Betätigung“ gemeint sind.

${ }^{19}$ Dies ist auch in der Rechtswissenschaft weithin unbestritten. Ungleichheiten in der Verteilung von Ressourcen und Lebenschancen gelten nicht schon per se als Verfassungsproblem. Vgl. etwa E.-W. Böckenförde (Fn. 18) Rn. 63, der nur „die Abwesenheit extremer wirtschaftlich-sozialer Gegensätze“"zu den Verfassungsvoraussetzungen zählt. Ähnlich H. Krüger (Fn. 18) 287; Ch. Starck in: H. v. Mangoldt/F. Klein/Ch. Starck (Hrsg.) GG I, 1999, Art. 3 Abs. 1 Rn. 6; A. Nußberger Voraussetzung oder Aufgabe des Staates? DVB1. 2008, 1081. Verschiedentlich wird aber angenommen, daß die Funktionsfähigkeit einer Verfassung in bestimmten Hinsichten „Gleichartigkeit“ bzw. - scheinbar synonym - „Gemeinsamkeit“ voraussetze. Für E.-W. Böckenförde ebd. Rn. 59 etwa hängt die Lebens- und Funktionsfähigkeit der Demokratie als Staats- und Regierungsform auch von bestimmten sozio-kulturellen Voraussetzungen ab. Sie betreffen die „Ordnungsstruktur und Verfaßtheit der Gesellschaft als solcher“ und die „geistig-bildungsmäßigen Gegebenheiten bei den Bürgern“. Dazu gehöre u.a. eine „relative Homogenität“, und dies wiederum sei eine „vor-rechtliche Gemeinsamkeit“" (Vorauflage: „Gleichartigkeit“) als „metarechtliche Grundlage demokratischer Gleichheit“, die sich „von dem ihr Fremden deutlich“ abgrenze (ebd. Rn. 63). Die vor-rechtliche Gleichartigkeit bzw. Gemeinsamkeit gründe sich auf ethnisch-kulturelle Eigenart und Tradition, auf gemeinsame politische Geschichte, gemeinsame Religion oder gemeinsames nationales Bekenntnis (ebd. Rn. 64). Andernfalls sei mit einem Übermaß an Dissoziationen und Antagonismen zu rechnen sowie mit Freund-Feind-Gruppierungen im Staatsin- 
Der Blick in die Nachbarwissenschaften gibt mir aber auch die Richtung für meine Untersuchung vor: Ich will wie die Soziologie an der Ungleichheit (nicht der Gleichheit) ansetzen, und ich will wie die politische Philosophie nach problematischen Fällen sozialer Ungleichheit suchen: Ich will erstens fragen, ob sozialpolitische Maßnahmen, die Ungleichheit verschärfen, grundrechtlich zu beanstanden sind. Ist dies nicht der Fall, ist die Verhinderung von sozialer Ungleichheit jedenfalls grundrechtlich nicht gefordert. Ich will zweitens fragen, ob das Gemeinschaftsrecht soziale Ungleichheit in Deutschland beeinflusst. Und ich will drittens fragen, ob die Verantwortung Deutschlands für Ungleichheit - wenn es sie gibt - auf das eigene Territorium begrenzt bleibt. Die dritte Frage bezieht sich auf völkerrechtliche Verantwortlichkeiten, die die innerstaatlichen ergänzen. So werden wir Ungleichheit auf verschiedenen Ebenen betrachten und an verschiedenen Maßstäben messen. Dabei wird sich zeigen, dass die Verbindung zwischen sozialer Ungleichheit und Recht nur lose ist. Die Frage nach der Verfassungsvoraussetzung stelle ich zurück.

nern, die ein gemeinsames Zusammenwirken verhinderten (ebd. Rn. 64). Kurz: Die Verfassung sei stabil nur bei substantieller Gleichheit der Bevölkerung. Die Anforderungen, die Habermas für den „Verfassungspatriotismus“ formuliert, zielen demgegenüber weniger auf einen inhaltlich bestimmten Wertekonsens als vielmehr auf einen Konsens über „das Verfahren legitimer Rechtsetzung und Machtausübung“. So J. Habermas Anerkennungskämpfe im demokratischen Rechtsstaat, in: Ch. Taylor Multikulturalismus und die Politik der Anerkennung, 1993, 147 (178f.). Eine „motivationale Verankerung“ der Verfassungswerte als einen „gemeinsamen Interpretationshorizont" hält jedoch auch $\mathrm{Ha}$ bermas für unabdingbar. Es ist nicht zu bestreiten, daß Antagonismen und religiös oder ethnisch definierte Freund-Feind-Gruppierungen in blutige Konflikte münden können. Die Verkoppelung von Beständigkeit der Verfassung einerseits und Gleichartigkeit der Menschen andererseits ist gleichwohl fragwürdig. Zum einen ist Systemzusammenhalt nachweisbar auch ohne Loyalität der Betroffenen möglich, ja, Integration kann sogar gegen den Willen der Betroffenen erfolgen. Integration funktioniert nämlich auch über Märkte oder Organisationen, insb. durch die Ausübung von Herrschaftsgewalt. Vgl. näher H. Esser Soziologie II (Fn. 13) 265 ff. Für das System - die Verfassung - folgt daraus nicht notwendig eine Gefahr. Illoyale Gesellschaftsmitglieder sind häufig apathisch und fragmentiert (Esser ebd. 139, 231, 254, 276, 293 ff.). Zum anderen ist der Zusammenhang zwischen Loyalität und religiöser oder ethnischer Vielfalt nicht zwingend „,negativ“. Loyalität von Einwanderern etwa kann ohne weiteres auch aus erfolgreichen Erwerbskarrieren folgen, also aus der Besetzung wichtiger Positionen in der Gesellschaft des Einwanderungslandes, z.B. im Sport, in der Kultur, der Wissenschaft oder der Wirtschaft. Anders gewendet: Loyalität ist nicht an die Überzeugung einer gemeinsamen Herkunft gebunden. Für die Systemtheorie wiederum schließt schon der Prozeß der Differenzierung die Herstellung von Einheit ein. Zur Systemdifferenzierung näher N. Luhmann Gesellschaft (Fn. 14) 595 ff. Danach ist die Integration des Systems keineswegs an Einheitsperspektiven oder Solidaritätserwartungen gebunden; Integration steht schlicht für „die Reduktion der Freiheitsgrade von Teilsystemen, die dies den Außengrenzen des Gesellschaftssystems und der damit abgegrenzten Umwelt des Systems verdanken“ (ebd. 602, 603). 


\section{Grundgesetz und soziale Ungleichheit}

\section{Beispiele}

Der Wohlfahrtsstaat gilt als zentraler Mechanismus der nationalstaatlichen Integration bzw. der Inklusion von Menschen in das politische System:20 Der Wohlfahrtsstaat war darauf angelegt, soziale Teilhabe zu sichern, wo andere Garanten nicht vorhanden waren. Insoweit war der Wohlfahrtsstaat nie neutral gegenüber sozialer Ungleichheit. Im Gegenteil, der Wohlfahrtsstaat war der Mechanismus zur Verringerung sozialer Ungleichheit. ${ }^{21}$ Umgesetzt wurde dies vor allem durch das Sozialrecht. ${ }^{22}$ Sind diese Annahmen korrekt, müssten wir an der rechtlichen

${ }^{20}$ M. Stolleis Geschichte des Sozialrechts in Deutschland, 2003; E. Eichenhofer Geschichte des Sozialstaats in Europa. Von der „sozialen Frage“ bis zur Globalisierung, 2007; F.-X. Kaufmann Varianten des Wohlfahrtsstaats. Der deutsche Sozialstaat im internationalen Vergleich, 2003; H. F. Zacher Bundesrepublik (Fn. 17); A. Hänlein/ F. Tennstedt in: B. B. v. Maydell/F. Ruland/U. Becker (Hrsg.) Sozialrechtshandbuch SRH, 2. Aufl. 2008, § 2; F.-X. Kaufmann Sozialstaatlichkeit unter den Bedingungen moderner Wirtschaft, in: F.-X. Kaufmann Sozialpolitik und Sozialstaat: Soziologische Analysen, 2002, 261. Die Systemtheorie beschreibt den Wohlfahrtsstaat als einen von zwei Modi der Inklusion von Menschen in das politische System. Näher N. Luhmann Politik (Fn. 14) $407 \mathrm{ff}$;; R. Stichweh Zur Theorie der politischen Inklusion, in: R. Stichweh (Fn. 14) $67 \mathrm{ff}$. Beide Inklusionsmodi sind danach prinzipiell auf Vollinklusion angelegt. Der eine Inklusionsmodus läuft über das Wahlrecht, der andere über die Instrumente des Wohlfahrtsstaates.

21 Zacher spricht wiederholt von der Herstellung von „mehr sozialer Gleichheit“ oder „mehr Gleichheit“. Vgl. z. B. H. F. Zacher Staatsziel (Fn. 17) Rn. 34, 86; H. F. Zacher Sozialstaat und Prosperität, FS Reiner Schmidt, 2006, 305.

22 E. Eichenhofer Sozialrecht 6. Aufl. 2007, Rn. 1: Recht zum „Schutz des Schwächeren“; H. F. Zacher Was ist Sozialrecht?, in: H. F. Zacher Abhandlungen zum Sozialrecht, hrsg. v. B. B. v. Maydell/E. Eichenhofer, 1993, 249 (251): „Recht mit gesteigerter Intensität des sozialpolitischen Gehalts“; G. Igl/F. Welti Sozialrecht. Ein Studienbuch, 8. Aufl. 2007, Rn. 1: „das der sozialen Gerechtigkeit und der sozialen Sicherheit dienende Recht“; ebenso St. Muckel Sozialrecht, 2. Aufl. 2007, Rn. 3. Der Ausbau der Wohlfahrtsstaatlichkeit nach dem Zweiten Weltkrieg führte in den 1970er Jahren zu einer Diskussion über die Angemessenheit eines (primär) liberalen Grundrechtsverständnisses. Vgl. z.B. H. F. Zacher Soziale Gleichheit. Zur Rechtsprechung des Bundesverfassungsgerichts zu Gleichheitssatz und Sozialstaatsprinzip, AöR 93 (1968) 341; K. H. Friauf Zur Rolle der Grundrechte im Interventions- und Leistungsstaat, DVB1. 1971, 674; U. Scheuner Die Funktion der Grundrechte im Sozialstaat. Die Grundrechte als Richtlinie und Rahmen der Staatstätigkeit, DÖV 1971, 505; P. Häberle Grundrechte im Leistungsstaat, VVDStRL 30 (1972) 43; W. Martens Grundrechte im Leistungsstaat, VVDStRL 30 (1972) 7; E.-W. Böckenförde Grundrechtstheorie und Grundrechtsinterpretation, NJW 1974, 1529; D. Wiegand Sozialstaatsklausel und soziale Teilhaberechte, DVB1. 1974, 657; P. Badura Das Prinzip der sozialen Grundrechte und seine Verwirklichung im Recht der Bundesrepublik Deutschland, Der Staat 14 (1975) 17; Ch. Starck 
Bearbeitung der jüngeren Reformen des Sozialrechts sehen können, inwieweit Ungleichheit als Rechtsproblem fassbar ist. Ich will dazu drei Beispiele diskutieren:

Das erste Beispiel entstammt der gesetzlichen Krankenversicherung. Das ist ein Bereich, der besonders stark vom Solidaritätsprinzip geprägt ist. ${ }^{23}$ Gleichwohl ist es nicht gelungen, die Verbindung zwischen Gesundheitszustand und sozio-ökonomischen Verhältnissen aufzubrechen: Je höher die Bildung, der Berufsstatus oder das Einkommen, umso geringer ist das Krankheitsrisiko. ${ }^{24}$ Die Verteilung von Gesundheit und Tod ist schichtspezifisch. ${ }^{25}$ Der Gesetzgeber hat die

Staatliche Organisation und staatliche Finanzierung als Hilfen zur Grundrechtsverwirklichung, in: Ch. Starck (Hrsg.) Bundesverfassungsgericht und Grundgesetz II, 1976, 480; W. Rüfner Grundrechtliche Leistungsansprüche, FS Georg Wannagat, 1981, 379.

${ }^{23}$ Das Recht der gesetzlichen Krankenversicherung richtet keine umfassende Solidargemeinschaft nach Maßgabe einer Einwohnerversicherung ein. Nicht beteiligt sind v.a. Besserverdienende, gesetzlich definiert als Beschäftigte, die die Jahresarbeitsentgeltgrenze überschreiten ( $\$ 6 \mathrm{Abs}$. 1 SGB V; derzeit: 48150 Euro im Jahr). Zudem endet die Beitragsbelastung an der Beitragsbemessungsgrenze (§ 223 Abs. 3 SGB V; derzeit: 43200 Euro im Jahr). Bei einem Beitragssatz von $14 \%$ - dies entspricht derzeit dem angenommenen durchschnittlichen Beitragssatz - liegt die (gesamte) Beitragslast höchstens bei 6048 Euro im Jahr.

${ }^{24}$ Nachweise in: Lebenslagen in Deutschland - Dritter Armuts- und Reichtumsbericht, BT-Drs. $16 / 9915$ v. 30. 6. 2008, 82ff.; European Commission Joint Report on Social Protection and Social Inclusion 2008. Social Inclusion, pensions, health care and long-term care, 2008, $67 \mathrm{ff}$;; A. Mielck Soziale Ungleichheit und Gesundheit. Einführung in die aktuelle Diskussion, 2005; M. Richter/K. Hurrelmann (Hrsg.) Gesundheitliche Ungleichheit. Grundlage, Probleme, Perspektiven, 2006; J. Siegrist/M. Marmot (Hrsg.) Soziale Ungleichheit und Gesundheit: Erklärungsansätze und gesundheitspolitische Folgerungen, 2008; J. P. Mackenbach Health Inequalities: Europe in Profile, 2006; M. Richter/K. Hurrelmann Warum die gesellschaftlichen Verhältnisse krank machen, APuZ 42/2007, 3; I. Ebsen Armut und Gesundheit, in: Armutsfestigkeit sozialer Sicherung, SDSRV 56, 2007, 133; K. A. Bender/St. Habermalz Are There Differences in the Health-Socio-economic Status Relationship over the Life Cycle? Evidence from Germany, LABOUR 22/1 (2008) 107.

${ }^{25}$ In den entwickelten Staaten ist die Lebenserwartung in den letzten 100 Jahren allgemein beträchtlich gewachsen. Der Anteil, um den die Mortalitätsrate der Schlechtergestellten die Mortalitätsrate der Bessergestellten übersteigt, war über die Zeit jedoch eher stabil, ja, das Risiko eines früheren Todes ist für Schlechtergestellte in den letzten Jahrzehnten sogar gewachsen. In den europäischen Staaten sind die Mortalitätsraten der Schlechtergestellten 1,5 bis 2,5 Mal so hoch wie die Mortalitätsraten der Bessergestellten. Die Morbiditätsrate erhöht sich für Schlechtergestellte europaweit um den Faktor 2 bis 4. Näher J. P. Mackenbach (Fn. 24) $5 \mathrm{ff}$., $20 \mathrm{ff}$. Für Deutschland stellt der letzte Armuts- und Reichstumsbericht der Bundesregierung (Fn. 24) 82 fest: „Der Anteil der Männer mit gesundheitlichen Beeinträchtigungen ist in der niedrigsten im Vergleich zur höchsten Bildungsgruppe um den Faktor 3,1 und bei Frauen um den Faktor 1,8 erhöht.“ Zu den Erklärungsansätzen näher M. Richter/K. Hurrelmann (Fn. 24) 6. 
Versicherten dennoch undifferenziert mit Zuzahlungen oder Leistungsbegrenzungen belastet. ${ }^{26}$ Davon sind seit dem 1. 1. 2004 auch Sozialhilfeempfänger nicht mehr ausgenommen. ${ }^{27}$

26 Der Gesetzgeber verfolgt seit Inkrafttreten des Sozialgesetzbuchs (SGB) Fünftes Buch (V) Gesetzliche Krankenversicherung v. 20. 12. 1988 BGB1. I S. 2477 (SGB V) verschiedene Strategien, um die Kostensteigerungen im Bereich der gesetzlichen Krankenversicherung in Grenzen bzw. die Beitragssätze stabil zu halten. Die Maßnahmen betreffen erstens das Verhältnis zwischen den Versicherten und ihren Krankenkassen (z.B. Einführung des Krankenkassenwahlrechts, Gesundheitsfonds). Hier geht es insbesondere um eine Stärkung des Wettbewerbs zwischen den einzelnen Kassen. Der Gesetzgeber versucht zweitens, vermutete Wirtschaftlichkeitsreserven zu erschließen (Einführung von Budgets, etwa im Bereich der Arzneimittel, Begrenzung der Zahl der zugelassenen Ärzte). Eine dritte Stoßrichtung der Maßnahmen ist die Leistungserbringung (z.B. Hausarztmodell, integrierte Versorgung, Zulassung von Einzelverträgen). Ein weiterer Teil der Maßnahmen zielt - viertens - auf die Leistungsansprüche der Versicherten. Hier geht es um Leistungsausschlüsse, Leistungsbegrenzungen und Zuzahlungen: Die konservativen Regierungen der 1990er Jahre konzentrierten sich auf die kieferorthopädische Behandlung (Ausschluß, wenn die Versicherten das 18. Lebensjahr vollendet haben, ausgenommen schwere Kieferanomalien) und den Zahnersatz (Ausschluß für Versicherte, die nach dem 31. 12. 1978 geboren sind; Festzuschüsse zum Zahnersatz). Die Zuzahlungen für Arznei- und Verbandmittel stiegen in den 1990er Jahren von 3, 5 und 7 DM auf 9, 11 und 13 DM. Die erste Reformmaßnahme der rotgrünen Koalition, das GKV-Solidaritätsstärkungsgesetz v. 19. 12. 1998 BGB1. I S. 3853, nahm dann wohl manche Leistungsbeschränkung zurück (kieferorthopädische Behandlung, Zahnersatz). Mit dem Gesundheits-Modernisierungsgesetz v. 14. 11. 2003 BGB1. I S. 2190 schnürte aber auch die rot-grüne Koalition ein Belastungspaket für die Versicherten: Dazu gehören etwa die Einführung einer Praxisgebühr ( $\$ 28$ Abs. 4), der Ausschluß weiterer Arzneimittel aus dem Leistungskatalog der Krankenkassen ( $\$ 34$ Abs. 1), der Ausschluß der Sehhilfen (§ 33 Abs. 1), die Abschaffung des Sterbegeldes, Leistungsbegrenzungen bei Sterilisation ( $\$ 24 \mathrm{~b}$ : durch Krankheit erforderlich) und bei medizinischen Leistungen zur Herbeiführung einer Schwangerschaft (§ 27a: Begrenzung auf drei Versuche, Begrenzung der Leistungspflicht auf 50\% der Kosten), die Beschränkungen bei den erstattungsfähigen Fahrkosten ( $\$ 60$ Abs. 1: zwingende medizinische Gründe), die Einführung eines besonderen, nur die Mitglieder treffenden Beitragssatzes für Zahnersatz (§ 58, später Gesetz zur Anpassung der Finanzierung von Zahnersatz v. 15. 12. 2004 BGBl. I S. 3445) und die Neuregelung der Pflicht zur Leistung von Zuzahlungen zu Arznei-, Heil- und Hilfsmittel (§ 61: 10 v.H. des Abgabepreises, mindestens 5 Euro, höchstens 10 Euro). Die erste Maßnahme der großen Koalition, das GKV-Wettbewerbsstärkungsgesetz v. 26. 3. 2007 BGBl. I S. 378, brachte den Versicherten einige punktuelle Leistungsverbesserungen, z. B. im Bereich der Gesundheitsförderung oder der palliativen Versorgung. Das Gesetz brachte jedoch auch Leistungsbegrenzungen, v.a. bei medizinisch nicht indizierten Maßnahmen. Viele der Reformen verwiesen die Versicherten auf ihre „Eigenverantwortung“ für die Gesundheit.

27 Das GMG 2003 regelte die für die Zuzahlungen geltende Belastungsgrenze neu. Bis dahin waren nach den Härtefallregelungen der $\S \S 61$ und 62 bestimmte Personengruppen von der Zuzahlungspflicht gänzlich befreit, z.B. Personen mit einem Bruttoein- 
Das zweite Beispiel ist der gesetzlichen Rentenversicherung entnommen. Die Rentenreform 2001 markiert den Übergang zu einer Alterssicherung auf mehreren Säulen. ${ }^{28}$ Wesentliches Element dieses Paradigmenwechsels war die Absenkung des Rentenniveaus. ${ }^{29}$ Lebens-

kommen unter 40 v.H. der monatlichen Bezugsgröße sowie Empfänger von Hilfe zum Lebensunterhalt. Teilweise befreit waren Versicherte, wenn die während eines Kalenderjahres entstandenen notwendigen Fahrkosten und die Zuzahlungen 2 v.H. der Bruttoeinnahmen zum Lebensunterhalt überstiegen. Das GMG 2003 hat die vollständige Befreiung von der Zuzahlungspflicht beseitigt. Die Belastungsgrenze liegt jetzt allgemein bei 2 v.H. der Bruttoeinnahmen zum Lebensunterhalt, für chronisch Kranke in Dauerbehandlung bei 1 v.H. Bei Empfängern von Hilfe zum Lebensunterhalt ist insoweit der Regelsatz des Haushaltsvorstandes maßgeblich. Das GKV-WSG 2007 beschränkte sodann die Privilegierung für chronisch kranke Menschen. Die niedrige Belastungsgrenze greift nur, wenn die Versicherten vor ihrer Erkrankung die einschlägigen Vorsorgeuntersuchungen regelmäßig in Anspruch genommen haben (§62 Abs. 1 S. 3 SGB V).

${ }^{28}$ Vgl. Sozialgesetzbuch (SGB) Sechstes Buch (VI) - Gesetzliche Rentenversicherung - v. 18. 12. 1989 BGBl. I S. $2261 \mathrm{idF}$ des Altersvermögensergänzungsgesetzes v. 21. 3. 2001 BGBl. I S. 403 und des Altersvermögensgesetzes v. 26. 6. 2001 BGBl. I S. 1310 („Riester-Rente“, „Eichel-Förderung“). Vgl. später auch die Änderungen durch das Alterseinkünftegesetz v. 5. 7. 2004 BGBl. I S. 1427 ( 10 Abs. 1 Nr. 2 EStG: „Rürup-Rente“), das RV-Nachhaltigkeitsgesetz v. 21. 7. 2004 BGBl. I S. 1791 und das Eigenheimrentengesetz v. 29. 7. 2008 BGBl. I S. 1509. Die Veränderungen in der Rentenpolitik werden verschieden beschrieben. Zum Teil wird der Wechsel von einer ausgabenorientierten Einnahmenpolitik zu einer einnahmenorientierten Ausgabenpolitik betont. So etwa M. G. Schmidt Rot-grüne Sozialpolitik (1998-2002), in: Ch. Egle/ T. Ostheim/R. Zohlnhöfer (Hrsg.) Das rot-grüne Projekt. Eine Bilanz der Regierung Schröder 1998-2002, 2003, 239 (248). Zum Teil wird die höhere Regulierungsverantwortung des Gesetzgebers herausgestrichen. In diesem Sinne P. Blömeke Die Regulierung nicht-staatlicher Alterssicherung. Deutschland und Großbritannien im Vergleich unter Berücksichtigung von Staatstheorie, Verfassungs- und Europarecht, 2007; L. Leisering Privatisierung der Alterssicherung als komplexe Ordnungsbildung. Zur Entstehung von Wohlfahrtsmärkten und regulativer Staatlichkeit, FS Ruland, 2007, 189; L. Leisering Soziale Regulierung privater Altersvorsorge in Deutschland. Instrumente, Normen und ordnungspolitische Herausforderungen, in: Deutsche Rentenversicherung Bund (Hrsg.) Alterssicherung im Mehr-Säulen-System: Akteure, Risiken, Regulierungen, 2008, 59.

${ }^{29}$ Die wesentliche Funktion der gesetzlichen Rente - die Lebensstandardsicherung wurde lange Zeit durch das Nettostandardrentenniveau ausgedrückt. Es lag im Jahr 2001 bei etwa $70 \%$ (F. Ruland Rentenversicherung, in: B. B. v. Maydell / F. Ruland [Hrsg.] Sozialrechtshandbuch [SRH], 3. Aufl. 2003, 958, Rn. 2, 70). Die Rentenreform 2001 und die Einführung des Nachhaltigkeitsfaktors werden das Rentenniveau bis zum Jahr 2030 deutlich absenken. Das Rentenniveau vor Steuern bzw. das steuerbereinigte Nettorentenniveau - dies ist nun der neue Maßstab - wird von derzeit 53\% auf $43 \%$ sinken (F. Ruland Die Alterssicherung als Teil der Lebensplanung, DRV 2006, 1 [4]). 
standardsicherung ist seither keine Aufgabe der gesetzlichen Rentenversicherung. ${ }^{30}$ Von Sommer 2003 bis Sommer 2007 wurden die Renten nicht erhöht. ${ }^{31}$ Über die Folgen der Änderungen wurde zunächst nur in

${ }^{30} \mathrm{Um}$ heute wenigstens eine Rente in der Höhe einer Grundsicherungsleistung (660 Euro) zu erhalten, sind etwa 25 Entgeltpunkte erforderlich. Ein Durchschnittsverdiener muß also etwas mehr als 25 Jahre in die gesetzliche Rentenversicherung einzahlen, damit die Rente das Grundsicherungsniveau erreicht. Wenn die Dämpfungsmaßnahmen der Jahre 2001 und 2004 voll wirksam sind, werden 30 Entgeltpunkte nötig sein, um einen Anspruch auf eine Rente zu erwerben, die der Grundsicherung entspricht. Zur Berechnung näher: Sachverständigenrat zur Begutachtung der gesamtwirtschaftlichen Entwicklung. Jahresgutachten 2007/08, 2007, 193f. Frauen in den alten Bundesländern erreichen armutsfeste Renten aus eigener Versicherung schon heute kaum. Der durchschnittliche Zahlbetrag der Rente liegt bei 492 Euro, die durchschnittlichen Entgeltpunkte pro Versicherungsjahr liegen bei 0,77 , und die durchschnittlichen Versicherungszeiten bei 26,3 Jahren (Deutsche Rentenversicherung Rentenversicherung in Zahlen 2008. Aktuelle Ergebnisse, Stand: 13. Juni 2008, 42f.). Wenn sich Erwerbsverhalten und Erwerbskarrieren nicht einschneidend ändern, wird der Anteil der Frauen, die von anderen Existenzsicherungen abhängig sind, in den alten Bundesländern weiter groß bleiben. Zur Einkommenssituation von Rentnern und Rentnerinnen näher U. Bieber/D. Klebula Erste Ergebnisse aus der Studie Alterssicherung in Deutschland 2003. Ergebnisse zur Vielschichtigkeit der monetären Situation im Alter, DRV 2005, 362; Th. Heien/K. Kortmann/Ch. Schatz Altersvorsorge in Deutschland 2005. Forschungsprojekt im Auftrag der Deutschen Rentenversicherung Bund und des Bundesministeriums für Arbeit und Soziales, 2007. Rechtspolitisch liegt der Rentenreform 2001 die Annahme zugrunde, daß Versicherte prinzipiell in der Lage sein werden, die Ausfälle aus der gesetzlichen Rentenversicherung über private Eigenvorsorge zu kompensieren. Zu den Kompensationsperspektiven näher A. Börsch-Suppan/T. BucherKoenen/A.Reil-Held/C. Wilke Zum künftigen Stellenwert der ersten Säule im Gesamtsystem der Alterssicherung, in: Deutsche Rentenversicherung Bund (Hrsg.) Alterssicherung im Mehr-Säulen-System: Akteure, Risiken, Regulierungen, DRV-Schriften 80 (2008) 13.

31 Vom 1. 7. 2003 bis 30. 6. 2007 lag der aktuelle Rentenwert bei 26,13 Euro (Westen) bzw. 22,97 Euro (Osten). Die zum 1. Juli eines jeden Jahres fällig werdende Anpassung wurde ausgesetzt, in den Jahren 2005 und 2006 allerdings zum Schutz der Rentner und Rentnerinnen. Die Anwendung der Formel für die Rentenanpassung hätte nämlich zu einer betragsmäßigen Verringerung der Renten geführt (Nachhaltigkeitsfaktor, Lohnentwicklung). Dies wurde 2005 durch die Schutzklausel des $\S$ 68a SGB VI verhindert, und 2006 durch das Gesetz über die Weitergeltung der aktuellen Rentenwerte ab 1. Juli 2006 v. 15. 6. 2006 BGB1. I S. 1304. Die nicht realisierten Negativanpassungen dürfen ab dem Jahr 2011 mit den zugelassenen Steigerungen verrechnet werden ( $\$ 68 \mathrm{a} \mathrm{iVm}$ § 255g SGB VI). Am 1. 7. 2007 wurde der aktuelle Rentenwert auf 26,27 Euro (Westen) bzw. 23,09 Euro (Osten) erhöht (RentenwertbestimmungsVO 2007 v. 14. 6. 2007 BGB1. I S. 1113). Seit 1. 7. 2008 liegt der aktuelle Rentenwert bei 26,56 bzw. 23,34 (vgl. Gesetz zur Rentenanpassung 2008 v. 26. 6. 2008 BGB1. I S. 1076). 
Fachkreisen gesprochen. ${ }^{32}$ Heute hat die Sorge vor einer drohenden Altersarmut auch die Öffentlichkeit erreicht. ${ }^{33}$

Das dritte Beispiel betrifft die Grundsicherung für Arbeitsuchende. Eine besondere Strenge des Gesetzes ergibt sich aus der rigiden Gestaltung der Geldleistungen. Das frühere Sozialhilferecht kombinierte bei der Hilfe zum Lebensunterhalt Regelsätze mit flexiblen einmaligen Leistungen für größere Aufwendungen. ${ }^{34}$ Das SGB II kombiniert feste Regelleistungen - für Alleinstehende heute 351 Euro - mit einem eng umgrenzten Katalog von einmaligen Leistungen. ${ }^{35}$ Die Inflexibilität führt in bestimmten Konstellationen zu Belastungen für die Betroffenen.

32 Vgl. z.B. den fünften Bericht zur Lage der älteren Generation, BT-Drs. 16/2190 v. 6. 7. 2006; W. Schmähl Die neue deutsche Alterssicherungspolitik und die Gefahr steigender Altersarmut, SozSich 55/12 (2006) 397; R. Himmelreicher/D. Frommert Gibt es Hinweise auf zunehmende Ungleichheit der Alterseinkünfte und zunehmende Altersarmut? Vierteljahrshefte zur Wirtschaftsforschung 75/1 (2006) 108; T. Mika/U. Bieber Verdeckte Armut der älteren Bevölkerung. Ausmaß von Niedrigeinkommen und Gründe der Nichtinanspruchnahme von Sozialhilfe unter Senioren, DRV 2006, 248; Ch. Wübbeke/A. Hirseland/S. Koch Das Altersarmutsrisiko von älteren Beziehern des Arbeitslosengelds II: Risikogruppen und Effekte des SGB II auf die Alterssicherung, in: Armutsfestigkeit sozialer Sicherung, SDSRV 56, 2007, 18; W. Strengmann-Kuhn Altersarmut in Deutschland - eine empirische Bestandsaufnahme und sozialpolitische Perspektiven, DRV 2008, 120. Die Bundesregierung weist den Vorwurf, daß die Rentenpolitik das Problem der Altersarmut verschärfen könnte, stets zurück (vgl. z.B. die Antworten auf die Kleinen Anfragen BT-Drs. 16/6935 v. 7. 11. 2007 und BT-Drs. $16 / 8634$ v. 19. 3. 2008).

${ }^{33} \mathrm{Vgl}$. aus der jüngsten Vergangenheit etwa: Altersarmut - in Hamburg müssen 16000 Rentner arbeiten, Die Welt, 31. 7. 2008, 30; Arbeitnehmerkammer kritisiert Rentenpolitik; Maßnahmen zur Umgestaltung des Rentensystems sollen Altersarmut verhindern und Lebensstandard sichern, taz, 1. 8. 2008, 24; Angst vor Altersarmut - Umfrage: Fast jeder Zweite erwartet Finanznöte, General-Anzeiger (Bonn), 8. 8. 2008, 16.

$34 \S \S 21,22$ Bundessozialhilfegesetz v. 30. 6. 1961 BGBl. I S. 815 (BSHG). Nach der beispielhaften Aufzählung in $\S 21$ Abs. 1a BSHG waren einmalige Leistungen insbesondere zu gewähren für die Beschaffung oder die Instandsetzung von Kleidung von nicht geringem Anschaffungspreis, für die Beschaffung von Brennmaterial, die Beschaffung von besonderen Lernmitteln für Schüler, die Instandsetzung von Hausrat, die Instandsetzung der Wohnung, die Beschaffung von Gebrauchsgütern von höherem Anschaffungswert oder für besondere Anlässe. Im übrigen war auch die Höhe der Regelsätze flexibel. In $\S 22$ Abs. 1 S. 1 BSHG hieß es wohl, daß laufende Leistungen zum Lebensunterhalt außerhalb von Anstalten nach Regelsätzen gewährt werden. § 22 Abs. 1 S. 2 BSHG ließ jedoch eine abweichende Bemessung zu.

${ }^{35}$ Näher § 23 Abs. 3 SGB II (abweichende Erbringung von Leistungen). Danach sind einmalige Leistungen nur vorgesehen für die Erstausstattung von Wohnungen, die Erstausstattung mit Bekleidung, mehrtägige Klassenfahrten. Allgemein sind die Leistungen des SGB II in § 19 umschrieben. Nach dem ersten Satz - nur so weit ist die Regelung von Interesse - erhalten erwerbsfähige Hilfebedürftige als Arbeitslosengeld II Leistungen zur Sicherung des Lebensunterhalts einschließlich der angemessenen Kosten für 
Alle Beispiele sind einschlägig. Die Sparmaßnahmen im Bereich der gesetzlichen Krankenversicherung führen wahrscheinlich zu einer Verschärfung der gesundheitlichen Ungleichheit, ${ }^{36}$ die „Versäulung“ der Alterssicherung - wegen der unterschiedlichen Deckung im Bereich der privaten Vorsorge - zu einer größeren Ungleichheit der Alterseinkommen, ${ }^{37}$ die Grundsicherung möglicherweise $\mathrm{zu}$ einer Bedarfsunterdeckung. ${ }^{38}$ Die Maßnahmen sind an verschiedenen Grundrechten zu messen: am Gleichheitssatz, an der allgemeinen Handlungsfreiheit, am Eigentumsrecht, am Recht auf ein Existenzminimum. Kommt es in den Beispielsfällen zu einer problematischen Form von Exklusion? ${ }^{39}$

Unterkunft und Heizung. Was die Regelleistung zur Sicherung des Lebensunterhalts im einzelnen umfaßt, ergibt sich aus § 20 Abs. 1 SGB II (Ernährung, Kleidung, Körperpflege, Hausrat, Haushaltsenergie ohne die auf die Heizung entfallenden Anteile, Bedarfe des täglichen Lebens sowie in vertretbarem Umfang auch Beziehungen zur Umwelt und eine Teilnahme am kulturellen Leben).

${ }^{36}$ M. Richter/K. Hurrelmann (Fn. 24) 7; Th. Gerlinger Gesundheitspolitik und die Frage der sozialen Ungleichheit in der gesundheitlichen Versorgung, in: K. Tiesmeyer/M. Brause/M. Lierse/M. Lukas-Nülle/Th. Hehlmann (Hrsg.) Der blinde Fleck. Ungleichheiten in der Gesundheitsversorgung, 2008, 39.

37 A. Börsch-Suppan/C. Wilke Zwischen Generationenvertrag und Eigenvorsorge. Wie Europa auf den demographischen Wandel reagiert, 2006, $51 \mathrm{ff}$.

${ }^{38}$ Kritisch etwa die Anträge BT-Drs. 16/696 v. 15. 2. 2006 (Bündnis 90/Die Grünen), BT-Drs. 16/2750 v. 27. 9. 2006 (Bündnis 90/Die Grünen), BT-Drs. 16/7040 v. 8. 11. 2007 (Die Linke), BT-Drs. 16/7113 v. 14. 11. 2007 (Bündnis 90/Die Grünen), BT-Drs. 16/7838 v. 23. 1. 2008 (Bündnis 90/Die Grünen), BT-Drs. 16/8761 v. 9. 4.2008 (Bündnis 90/Die Grünen).

${ }^{39}$ Der Begriff der Exklusion hat keine besonders lange Vergangenheit. Er wurde im politischen Diskurs erstmals in Frankreich verwendet (dazu im einzelnen L. Leisering Desillusionierungen des modernen Fortschrittsglaubens. „Soziale Exklusion“ als gesellschaftliche Selbstbeschreibung und soziologisches Konzept, in: Th. Schwinn [Hrsg.] Differenzierung und soziale Ungleichheit, 2004, $238 \mathrm{ff}$.). Der Begriff fand dann aber rasch Eingang in die gemeinschaftsrechtliche Sozialpolitik und ist dort gar nicht mehr wegzudenken. Er hat auf der Gemeinschaftsebene erfolgreich den Armutsbegriff verdrängt; vgl. zunächst die Entschl. des Rates v. 29. 9. 1989 über die Bekämpfung der sozialen Ausgrenzung, ABl. Nr. C 277/1, später Beschl. Nr. 50/2002/EG v. 7. 12. 2001 zur Einführung eines Aktionsprogramms der Gemeinschaft zur Förderung der Zusammenarbeit der Mitgliedstaaten bei der Bekämpfung der sozialen Ausgrenzung, ABl. 2002 Nr. L 10/1. Der Exklusionsbegriff weist eine Reihe von Unschärfen auf, die es schwierig machen, die erfaßten Phänomene genauer zu umschreiben oder Exklusion von Armut abzugrenzen. In der Soziologie wird Exklusion daher häufig näher qualifiziert. So etwa bei $R$. Stichweh Inklusion/Exklusion, funktionale Differenzierung und die Theorie der Weltgesellschaft, in: R. Stichweh (Fn. 14) 45: „Exklusion erweist sich ... als ein multidimensionaler, kumulativer und sequentiell vernetzter Vorgang eines Ausschlusses aus einer Mehrzahl von Funktionssystemen“. 


\section{Gesundheit und Ungleichheit}

Die Rechtsprechung des BSG zur gesetzlichen Krankenversicherung bietet viel Anschauungsmaterial für Versuche, soziale Ungleichheit, genauer: unterschiedliche Wirtschaftskraft, grundrechtlich zu thematisieren. $\mathrm{Zu}$ prüfen war der Ausschluss von Leistungen, etwa bei der kieferorthopädischen Behandlung oder bei Arzneimitteln. ${ }^{40}$ Auch Leistungsbegrenzungen standen auf dem Prüfstand, so etwa die Begrenzung der Kostenübernahme bei künstlichen Befruchtungen auf $50 \%{ }^{41}$ Empfänger von Sozialhilfe machten geltend, daß sie nicht zu Zuzahlungen verpflichtet werden dürften..$^{42}$

\section{a) Gleichheitsrecht}

Unter dem Gleichheitssatz stehen Klägern zwei Argumentationsstränge zur Verfügung: Sie sind entweder von einer belastenden allgemeinen Regel betroffen (alle müssen Zuzahlungen leisten, bestimmte Arzneimittel sind vom Leistungskatalog generell ausgenommen). Dann können die Kläger einwenden, für sie hätte ein Ausnahmetatbestand geschaffen werden müssen. ${ }^{43}$ Oder die Betroffenen sind schlechter gestellt als andere (als unter 18-Jährige, als diejenigen, für die eine Härteklausel

40 Zum Ausschluß der über 18-Jährigen von der kieferorthopädischen Behandlung vgl. BSG, Urteil v. 9. 12. 1997, 1 RK 11/97; zuvor auch schon BSG, Urteil v. 6. 6. 1991, 3 RK 12/90; zum Ausschluß von Arzneimitteln vgl. BSG, Urteil v. 10. 5. 2005, B 1 KR 25/03 R (Viagra); BSG, Urteil v. 18. 7. 2006, B 1 KR 10/05 R (Caverject), bestätigt durch den Beschluß des BVerfG v. 28. 2. 2008, 1 BvR 1778/05.

${ }^{41}$ BSG, Urteil v. 16. 12. 2003, B $1 \mathrm{KR}$ 12/02 R (Mutter-Kind-Kur); BSG, Urteil v. 19. 9. 2007, B 1 KR 6/07 R (künstliche Befruchtung, Begrenzung der Leistungen auf $50 \%$ der Gesamtkosten verfassungsmäßig) und dazu die Urteilsanmerkung von J. Beck $\mathrm{SGb} 2008,356$ (359). Zu § 27a SGB V vgl. auch BVerfG, Urteil v. 28. 2. 2007, 1 BvL 5/03 (Beschränkung des Anspruchs auf Personen, die miteinander verheiratet sind, verfassungsmäßig).

42 BSG, Urteil v. 12. 10. 1988, $3 / 8$ RK 15/87; BSG, Urteil v. 22. 2. 1989, 8/5a RKn 23/87; BSG, Urteil v. 29. 10. 1991, 13/5 RJ 36/90.

${ }^{43}$ Zur verfassungsrechtlichen Bedeutung von Härteklauseln vgl. z.B. BVerfGE 33, 303 (348); 37, 154 (164f.), 102, 254 (336). Daß Art. 3 Abs. 1 GG - bei wesentlich unterschiedlichen Sachverhalten - auch ein Recht auf Ungleichbehandlung vermittelt, ist weitgehend akzeptiert. Zugleich wird aber festgehalten, daß das Recht nicht jeden Unterschied abbilden müsse. Näher R. Bergmann in: D. Hömig (Hrsg.) GG, 8. Aufl. 2007, Art. 3 Rn. 5; W. Heun in: H. Dreier (Hrsg.) GG I, 2. Aufl. 2004, Art. 3 Rn. 19 und 24; H. D. Jarass in: H. D. Jarass/B. Pieroth, GG, 8. Aufl. 2006, Art. 3 Rn. 5; L. Osterloh in: M. Sachs (Hrsg.) GG, 4. Aufl. 2007, Art. 3 Rn. 83; B. Pieroth/B. Schlink Grundrechte. Staatsrecht II, 24. Aufl., 2008, Rn. 436f.; Ch. Starck (Fn. 19) Art. 3 Abs. 1 Rn. 10; R. Alexy Theorie der Grundrechte, 1985, 370. 
zum Tragen kommt). Dann können die Betroffenen behaupten, die Schlechterstellung hätte keinen sachlichen Grund.

Beide Argumentationsstränge sind vom BSG grundsätzlich akzeptiert. ${ }^{44}$ Erfolgreich sind die Einwände aber nicht. Dem Recht auf Ungleichbehandlung setzt das BSG mehreres entgegen: Der Gesetzgeber dürfe den Leistungskatalog der gesetzlichen Krankenversicherung dessen ungeachtet nach finanzwirtschaftlichen Erwägungen bestimmen. ${ }^{45}$ Oder: Der Gesetzgeber sei zu einer differenzierenden Regelung nur verpflichtet, wenn die betroffene Gruppe so groß sei, daß der Gesetzgeber keine typisierende Regelung hätte treffen dürfen. ${ }^{46}$ Oder, unlängst: Die auch die Empfänger von Arbeitslosengeld II treffende Zuzahlungspflicht ziele auf eine Verbesserung der Belastungsgerechtigkeit und eine Steuerung des Verbraucherverhaltens; dies sei verfassungsmäßig. ${ }^{47}$ Das Recht auf Gleichbehandlung wiederum läuft regelmäßig leer, weil das BSG die angegriffene Regelung mit dem Hinweis auf bestehende Unterschiede für gerechtfertigt erklärt. ${ }^{48}$

44 Zum Recht auf Gleichbehandlung z.B. BSG, Urteil v. 3. 3. 1994, 1 RK 33/93, Rn. 23 (Zuzahlungen zu Arzneimitteln). Zum Recht auf Ungleichbehandlung ausdrücklich BSG, Urteil v. 22. 2. 1989, 8/5a RKn 23/87, Rn. 13 (Zuzahlungen zur Krankenhauspflege); BSG, Urteil v. 9. 6. 1998, B 1 KR 17/96 R, Rn. 15 (Befreiung von der Zuzahlungspflicht; lebensnotwendiges Arzneimittel); BSG, Urteil v. 19. 9. 2007, B 1 KR 6/07 R, Rn. 17 (künstliche Befruchtung; Begrenzung des Leistungsanspruchs auf $50 \%$ der Kosten).

45 Vgl. BSG, Urteil v. 19. 9. 2007, B 1 KR 6/07 R, Rn. 18 (künstliche Befruchtung; Begrenzung des Leistungsanspruchs auf 50\% der Kosten) mit Hinweisen auf Entscheidungen des BVerfG, die die Zumutbarkeit von Eigenleistungen für Versicherte allerdings nicht bestätigen.

46 BSG, Urteil v. 8. 6. 1994, 3/1 RK 54/93, Rn. 19 (Leistungsausschluß von Hörgerätebatterien). Der 1921 geborene Kläger war hilfebedürftig und lebte in einem Pflegeheim. Er konnte sich die Batterien nicht selbst finanzieren. Dies spielte keine Rolle. Denn: „Die Gruppe der auf Sozialhilfe angewiesenen, insbesondere auf Kosten des Sozialhilfeträgers in Pflegeheimen untergebrachten Personen, die außerdem noch besonders hohe Kosten für Hörgerätebatterien aufzuwenden haben, ist nicht so groß und so typisch, daß sie der Gesetzgeber im Rahmen der typisierenden Regelung von Massenerscheinungen wie die Jugendlichen behandeln und von der allgemeinen Regelung ausnehmen mußte“.

47 BSG, Urteil v. 22. 4. 2008, B 1 KR 10/07 R, Rn. 27.

48 Problematisch nochmals BSG, Urteil v. 8. 6. 1994, 3/1 RK 54/93, Rn. 19 (Leistungsausschluß von Hörgerätebatterien) mit dem Hinweis darauf, daß Jugendliche in aller Regel über geringe Einkünfte verfügten. Dann fährt das BSG fort: „Demgegenüber handelt es sich bei dem typischen Hörgeräteträger um eine ältere Person mit zum Lebensunterhalt ausreichendem Einkommen, sei es auch in Form einer Rente, und lediglich einohriger Versorgung." An der Stichhaltigkeit dieser Aussage kann man zweifeln. 
Die Argumente des BSG halten sich im Rahmen der Grundrechtsdogmatik. Schon nach der alten Willkür-Formel ${ }^{49}$ hat das BVerfG nicht nur Vergleichsgruppen und Vergleichsperspektiven betrachtet (z.B. Schutzbedürftigkeit), sondern auch ein gleichheitsfremdes Argument zugelassen: Die Notwendigkeit einer typisierenden Regelung könne die Ungleichbehandlung in den Hintergrund treten lassen..$^{50}$ Härtefälle oder Unterschiede in der Wirtschaftskraft seien hinzunehmen. ${ }^{51}$ Auch nach der neuen Formel 52 ist das Feststellen einer Ungleichbehandlung nur der erste Schritt. Die Feststellung macht rasch der Frage Platz, ob die Regelung einen legitimen Grund hat, und der muss sich nicht spezifisch auf Unterschiede zwischen den Vergleichsgruppen beziehen. ${ }^{53}$ So kann die Gleichheitsprüfung von Finanzierungsinteressen, Solidaritätsaspekten oder Fragen der Belastungsgerechtigkeit dominiert werden. ${ }^{54}$ Unter

${ }^{49}$ Nach der alten Formel gebietet der allgemeine Gleichheitssatz dem Gesetzgeber, wesentlich Gleiches gleich und wesentlich Ungleiches ungleich zu behandeln (so z. B. BVerfGE 98, 365 [385]).

50 BVerfGE 23, 135 (144); 103, 271 (290f.); 103, 392 (401f.); 117, 316 (328).

51 Vgl. St. Huster Gleichheit und Verhältnismäßigkeit. Der allgemeine Gleichheitssatz als Eingriffsrecht, JZ 1994, 541, und St. Huster Rechte und Ziele. Zur Dogmatik des allgemeinen Gleichheitssatzes, 1993, 165 ff., der zwischen den Anforderungen des Gleichheitssatzes und den Zweckmäßigkeitserwägungen der „Typisierungs-Rechtsprechung“ explizit ein Spannungsverhältnis sieht.

${ }^{52}$ Nach der neuen Formel ist Ungleichbehandlung nur zulässig, wenn Unterschiede von solcher Art und solchem Gewicht bestehen, daß sie die ungleiche Behandlung rechtfertigen können (so z.B. BVerfGE 113, 167 [214f.]). Diese Gleichheit wird manchmal als verhältnismäßige Gleichheit bezeichnet.

53 BVerfGE 89, 365 (376); 113, 167 (214ff.); ferner BVerfG, Nichtannahmebeschluß v. 7. 4. 2008 , 1 BvR 1924/07 = DVB1. 2008, 847; BVerfG, Nichtannahmebeschluß v. 28.2. 2008, 1 BvR 2137/06 = DVB1. 2008, 645.

54 Genau genommen, wird die Gleichheitsprüfung in der gerichtlichen Praxis mehrfach mit Unwägbarkeiten belastet. Erstens wird wenig darüber reflektiert, mit welcher (empirischen) Verläßlichkeit die Unterschiede zwischen den Vergleichsgruppen festzustellen sind. Um noch einmal auf das Beispiel der Leistungskürzung bei den Hörgerätebatterien zurückzukommen (Fn. 46 und 48): Ist der sachliche Grund für die Begünstigung von Jugendlichen wirklich dargetan, wenn das Gericht behauptet, der typische Hörgeräteträger sei eine ältere Person mit ausreichendem Einkommen? Zweitens bleibt die Rolle der Gerechtigkeit unklar. Verschiedentlich geht das BVerfG davon aus, daß der Gleichheitssatz die Gerechtigkeit definiert (so z. B. BVerfGE 15, 126 [146]). Zumeist hat Gerechtigkeit aber die Funktion eines externen Maßstabes für die Gleichheitsprüfung (vgl. z.B. BVerfGE 115, 381 [389]). In solchen Fällen geht die Gleichheitsprüfung unvermittelt in eine Gerechtigkeitsprüfung über: Ob eine Regelung dem Gleichheitssatz entspricht, hängt dann nämlich davon $\mathrm{ab}$, ob sie gerecht ist oder nicht. Damit kann das BVerfG - und dies ist wohl kein Zufall - anknüpfen an G. Leibholz Die Gleichheit vor dem Gesetz. Eine Studie auf rechtsvergleichender und rechtsphilosophischer Grundlage, 2. Aufl. 1959. Leibholz bestimmt den materiellen Gehalt des Gleichheitssatzes in 
dem Aspekt der sozialen Ungleichheit ist ein Leistungsausschluss sogar besonders schwer anzugreifen. Die Betroffenen können nicht behaupten, ihnen gegenüber hätte der Leistungsausschluss nicht erfolgen dürfen, weil sie - anders als andere Ausgeschlossene - niedrige Einkommen hätten. Sie würden damit einen originären Leistungsanspruch geltend machen und dafür bietet Art. 3 Abs. 1 GG keine Grundlage. Die Betroffenen müssen sich mit Versicherten vergleichen, die Leistungen beziehen und behaupten, sie müssten genauso behandelt werden wie diese. Eine solche Gleichheitsprüfung kann aber schon daran scheitern, dass es keine plausible Vergleichsgruppe gibt. Im Übrigen müsste sich die Gleichheitsprüfung mit der Frage auseinandersetzen, ob die Leistung A aus der Sicht der Krankenversicherung anders behandelt werden darf als die Leistung B. Auf soziale Ungleichheit ist dabei nicht zwingend einzugehen.

Meine zweite These lautet deshalb: Im Gesundheitsbereich kann die Vergrößerung von sozialer Ungleichheit nur ausnahmsweise gleichheitsrechtlich angegriffen werden. Der Maßstab des Gleichheitssatzes ist zum Teil zu grob, zum Teil ungeeignet.

\section{b) Menschenwürde}

Erfolgversprechender scheint der Ansatz aus Art. 1 Abs. 1 GG iVm dem Sozialstaatsprinzip, auch vor dem BSG. Denn mit Blick auf Zuzahlungen betont das BSG seit langem, dass Menschen mit keinem oder wenig Einkommen nicht gezwungen werden dürften, auf eine Krankenbehandlung zu verzichten. ${ }^{55}$ Das Existenzminimum sei jedenfalls $\mathrm{zu}$

der Tat mit Hilfe des Gerechtigkeitsbegriffes (ebd. 45, 72). Er ist sich aber bewußt, daß der Gerechtigkeitsbegriff bzw. der Willkürbegriff nicht allgemeingültig definiert werden könne; maßgeblich sei daher in beiden Fällen das „Rechtsbewußtsein einer Gemeinschaft" (ebd. 60, 61). Das BVerfG dagegen hat nie offengelegt, welche Gerechtigkeitsnorm es im Sinne hat. Das macht die Gleichheitsprüfung an einer entscheidenden Stelle intransparent. Drittens bleibt unklar, nach welchen Regeln die vergleichende Betrachtung (sind A und B wesentlich gleich oder ungleich?) durch eine abwägende Betrachtung (rechtfertigt die Verfolgung des Ziels A eine sonst unzulässige Ungleichbehandlung?) ersetzt werden darf. Die in der Rechtswissenschaft angebotene Unterscheidung von „Rechten“ und „Zielen“ macht das Problem sichtbar, gibt aber keine Antwort darauf, unter welchen Voraussetzungen sich Betroffene damit zufrieden geben müssen, daß ihr Gleichheitsrecht mit Nützlichkeitsüberlegungen konfrontiert wird. Vgl. dazu insb. St. Huster (Fn. 51); St. Huster in: K. H. Friauf/W. Höfling (Hrsg.) Berliner Kommentar, 2002, Art. 3.

55 BSG, Urteil v. 12. 10. 1988, 3/8 RK 15/87, Rn. 13 (Eigenbeteiligung an Krankenhauskosten). 
wahren. ${ }^{56}$ Freilich, in der Frage, was das Existenzminimum ist, hielt sich das BSG immer bedeckt. Zunächst orientierte sich das BSG erkennbar an den Regelsätzen der Sozialhilfe. ${ }^{57}$ Im April 2008 vertrat das BSG einen neuen Ansatz: Es akzeptierte die Zuzahlungspflicht für Empfänger von Arbeitslosengeld II. ${ }^{58}$ Das BSG unterschied zwischen den „denkbar untersten“ verfassungsrechtlichen Grenzen und anderen Mindestvoraussetzungen für ein menschenwürdiges Dasein. ${ }^{59} \mathrm{Zu}$ den denkbar untersten Grenzen gehöre lediglich das zur physischen Existenz Notwendige. Da die Zuzahlungspflichten des SGB V diese Untergrenze nicht berührten, seien die Regelungen verfassungsrechtlich unbedenklich.

Die neue Position des BSG ist in mancherlei Hinsicht kritikwürdig: Das BSG argumentiert an einem entscheidenden Punkt mit einer petitio principii, ${ }^{60}$ das BSG bricht mit der Rechtsprechung des BVerwG, die für das Sozialhilferecht stets von einem sozio-kulturellen Existenzminimum ausgegangen war, ${ }^{61}$ und das BSG erweckt zu Unrecht den Eindruck, als könnte es sich auf die Rechtsprechung des BVerfG stützen. ${ }^{62}$ Dennoch:

56 BSG, Urteil v. 29. 10. 1991, 13/5 RJ 36/90, Rn. 16 (Eigenbeteiligung an Krankenhauskosten); BSG, Urteil v. 3. 3. 1994, 1 RK 33/93, Rn. 26 (Zuzahlung zu Arzneimitteln).

57 BSG, Urteil v. 29. 10. 1991, 13/5 RJ 36/90, Rn. 16 (Eigenbeteiligung an Krankenhauskosten); BSG, Urteil v. 8. 6. 1994, 3/1 RK 54/93, Rn. 18 (Leistungsausschluß von Hörgerätebatterien); BSG, Urteil v. 25. 10. 1994, 3 RK 16/94, Rn. 36 (Leistungsausschluß von Hörgerätebatterien).

58 BSG, Urteil v. 22. 4. 2008, B 1 KR 10/07 R, und Urteil v. 22. 4. 2008, B 1 KR 18/07 R. Empfänger von Arbeitslosengeld II unterliegen grundsätzlich der Versicherungspflicht nach $\S 5$ Abs. 1 Nr. 2a SGB V. Auf der Basis von derzeit 28,58 Euro kalendertäglich (vgl. § 232a Abs. 1 Nr. 2 SGB V) ist der Bund zur Tragung der Beiträge verpflichtet (§ 251 Abs. 4 SGB V).

59 BSG, Urteil v. 22. 4. 2008, B 1 KR 10/07 R, Rn. $31 \mathrm{ff}$.

${ }^{60} \mathrm{Daß}$ der Gesetzgeber mit der Regelleistung des SGB II nach $§ 20$ Abs. 2 SGB II nicht nur die physische Existenz sichern wolle, sondern mehr, schließt das BSG aus dem Umstand, daß andere Gesetze ( $\$ 26$ SGB XII, AsylbLG) niedrigere Leistungen zulassen (BSG, Urteil v. 22. 4. 2008, B 1 KR 10/07 R, Rn. 49). Dieser Hinweis übersieht zum einen, daß es nach dem verfassungsrechtlichen Maßstab nicht darauf ankommt, was der Gesetzgeber will, sondern was er tut. Zum anderen folgt aus der Absenkung von Leistungen in anderen Kontexten nicht, daß die Regelleistung des SGB II in jedem Fall existenzsichernd ist. Um diese Frage zu beantworten, benötigt man einen eigenständigen Standard, an dem dann sowohl die Leistungen des SGB II als auch die des SGB XII oder des AsylbLG zu messen sind.

61 Z.B. BVerwGE 97, 376 (378) - Klassenfahrt, m.w.N.

62 Nach BVerfGE 45, 187 (228f.) gehört zu einem menschenwürdigen Dasein auch die Aussicht darauf, nach Verbüßung einer Freiheitsstrafe wieder in Freiheit zu gelangen. BVerfGE 76, 143 (159) und BVerfGE 81, 58 (66) ordnen der Menschenwürde ein „religiöses Existenzminimum“ zu, denn maßgeblich seien die Grundbedürfnisse von Menschen als „sittliche Personen“. Von einem „sozialen Wert- und Achtungsanspruch“ 
Unvertretbar scheint mir die Position des BSG nicht. Zum einen hat das BVerfG stets vermieden, den „obersten Wert“ des Verfassungsrechts ${ }^{63}$ näher zu beschreiben. Wir wissen, dass danach die Mindestvoraussetzungen für ein menschenwürdiges Dasein zu sichern sind. ${ }^{64}$ Aber das ist nur eine Paraphrase des Art. 1 Abs. 1 S. 1 GG. Wir wissen, dass die Mindestvoraussetzungen raum- und zeitgebunden sind. ${ }^{65}$ Das ist eine Selbstverständlichkeit. Und wir wissen, dass die Regelsätze des Sozialhilferechts eine Richtschnur für die Auslegung der Menschenwürdegarantie sind. ${ }^{66}$ Dieser letzte Hinweis war methodisch immer fragwürdig. ${ }^{67}$ Zum anderen hat auch die Rechtswissenschaft kein kohärentes Verständnis entwickelt. Es besteht keine Einigkeit über den Sitz des Rechts auf Existenzminimum, und es gibt keine konsensfähige Dogmatik zum wirtschaftlichen Existenzminimum. ${ }^{68}$ Das BSG vergrößert demnach die Gruppe derer, die „Existenzminimum“ ohnedies eng verstehen.

spricht schließlich auch noch BVerfGE 117, 71 (89). Die angesprochenen Aspekte gehen über ein physisches Existenzminimum hinaus.

63 BVerfGE 27, 1 (6).

64 BVerfGE 40, 121 (133); 44, 353 (375); 82, 60 (80); 87, 153 (161); 110, 412 (445f.). In BVerfGE 1, 97 (104) hatte das BVerfG noch gemeint, Art. 1 Abs. 1 GG gebiete nicht den Schutz vor materieller Not, sondern den Schutz vor Angriffen durch Dritte (Erniedrigung, Brandmarkung, Ächtung).

65 Z.B. BVerfGE 87, 153 (172): „realitätsgerecht“.

66 Z.B. BVerfGE 99, 246 (259).

${ }^{67}$ Das einfachgesetzliche Sozialhilferecht und die auf dieser Grundlage ergangenen Verordnungen über die Regelsätze geben nur dann einen Maßstab für die verfassungsrechtliche Pflicht zur Sicherung eines menschenwürdigen Daseins ab, wenn verläßlich angenommen werden dürfte, daß dieses Regelwerk der Verfassung tatsächlich entspricht. Eine solche Annahme hat das BVerfG nie zu begründen versucht. Kritisch auch St. Huster Medizinische Versorgung im Sozialstaat: Zur Bedeutung des Sozialhilferechts für die Bestimmung einer medizinischen Mindestversorgung, in: N. Mazouz/M. H. Werner/U. Wiesing (Hrsg.) Krankheitsbegriff und Mittelverteilung, 2004, 157 (164); W. G. Leisner Existenzsicherung im öffentlichen Recht. Minimum - Grundlagen - Förderung, 2007, 132.

68 Vgl. H. Dreier in: H. Dreier (Hrsg.) GG, 2. Aufl. 2004, Art. 1 I Rn. 158; G. Dürig in: Th. Maunz/G. Dürig, GG, 1958, Art. 1 Abs. 1 Rn. 43 f. und Art. 2 Abs. 2 Rn. 27; M. Herdegen in: Th. Maunz/G. Dürig, GG, 2005, Art. 1 Abs. 1 Rn. 114; U. Di Fabio in: Th. Maunz/G. Dürig, GG, 2004, Art. 2 Abs. 2 Rn. 45; W. Höfling in: M. Sachs (Hrsg.) GG, 4. Aufl. 2007, Art. 1 Rn. 31; M. Enders in: K. H. Friauf/W. Höfling (Hrsg.) Berliner Kommentar, 2005, Art. 1 Rn. 114f.; H. D. Jarass in: H. D. Jarass/B. Pieroth, GG, 8. Aufl. 2006, Art. 1 Rn. 3, 11, 15 b und Art. 2 Rn. 94; D. Murswiek in: M. Sachs, ebd., Art. 2 Rn. 224 ff.; A. Podlech in: AK-GG I, 3. Aufl. 2001, Art. 1 Abs. 1 Rn. 25 ff., 63; Ch. Starck in: H. v. Mangoldt/F. Klein/Ch. Starck (Hrsg.) GG I, 4. Aufl. 1999, Art. 1 Abs. 1 Rn. 36, 40; H. Schulze-Fielitz in: H. Dreier, ebd., Art. 2 II Rn. 96; Pieroth/B. Schlink (Fn. 43) Rn. 361 f.; W. Cremer Freiheitsgrundrechte. Funktionen und Strukturen, 2003, $360 \mathrm{ff}$;; O. Bachof Begriff und Wesen des sozialen Rechtsstaates, VVDStRL 12 (1954) 37; 
Hinter der engen Auslegung des Art. 1 Abs. 1 GG steht zum einen der Wunsch nach Klarheit eines finanzwirksamen Leistungsanspruchs gegenüber dem Staat, zum anderen die Sorge, Banalitäten könnten zum Verfassungsstreit werden. ${ }^{69}$ Ich bin nicht sicher, dass den Bedenken nur durch eine enge Auslegung Rechnung getragen werden kann. Denn auch das weite Verständnis betont den dynamischen Charakter der Menschenwürdegarantie. ${ }^{70}$ Der Leistungsanspruch kann schmäler werden. Viel wichtiger ist, dass ein enges Verständnis nicht nur für das wirtschaftliche Existenzminimum gelten kann. Jedes Mal, wenn der Gesetzgeber Leistungen der gesetzlichen Krankenversicherung in die Eigenverantwortung der Betroffenen überträgt, kann dies zugleich bedeuten: Sie sind für Hilfebedürftige unerschwinglich, ohne dass der Sozialhilfeträger einspringen darf. ${ }^{71} \mathrm{Zu}$ fragen ist daher auch nach dem

G. Dürig Verfassung und Verwaltung im Wohlfahrtsstaat, JZ 1953, 193; H. M. Heinig Menschenwürde und Sozialstaat, in: P. Bahr/H. M. Heinig (Hrsg.) Menschenwürde in der säkularen Verfassungsordnung. Rechtswissenschaftliche und theologische Perspektiven, 2006, 251; W. G. Leisner (Fn. 67) 98ff.; E.-W. Luthe/F. Dittmar Das Existenzminimum der Gegenwart, SGb 2004, 272; V. Neumann Menschenwürde und Existenzminimum, NVwZ 1995, 426; V. Neumann Sozialstaatsprinzip und Grundrechtsdogmatik, DVBl. 1997, 92; J. M. Soria Das Recht auf Sicherung des Existenzminimums, JZ 2005, 644; T. M. Spranger Der sozialhilferechtliche Anspruch auf das Existenzminimum aus verfassungsrechtlicher Sicht, VerwRdSch 1999, 242; M. Wallerath Zur Dogmatik eines Rechts auf Sicherung des Existenzminimums, JZ 2008, 157. Cremer, Dreier, Höfling, Jarass, Pieroth/Schlink, Soria, Starck und Wallerath plädieren für eine Verankerung des Rechts auf ein Existenzminimum primär in Art. 1 Abs. 1 GG, Neumann für eine Verankerung in Art. 3 Abs. 1 GG, Bachof, Di Fabio, Dürig und Murswiek nennen Art. 2 Abs. 2 S. 1 GG, Enders, Luthe und Dittmar Art. 20 Abs. 1 GG; G. W. Leisner will sich wohl nicht festlegen. Die Wahl der Rechtsgrundlage beeinflußt zumindest in gewissem Umfang den für erforderlich erachteten Standard: Wer Art. 2 Abs. 2 S. 1 GG für einschlägig hält, plädiert eher für ein physisches Existenzminimum (Bachof, Di Fabio, Dürig; Murswiek). Im übrigen gehen die Meinungen weit auseinander: Sie reichen von der staatlichen Pflicht zur Gewährleistung eines soziokulturellen Existenzminimums, das sich nach den herrschenden Lebensgewohnheiten (Cremer) oder den unteren Lohngruppen (Neumann) bestimmen soll bis zur Beschränkung der Pflicht auf ein physisches Minimum (Enders; Heinig; Jarass; Soria; Spranger; Starck; Höfling: „Menschenwürdesockel des Leistungsrechts“; Pieroth/Schlink: „Verkommenlassen in hilfloser Lage“). Zum Teil wird die Festsetzung des Existenzminimums überhaupt dem einfachen Gesetzgeber überlassen, dem durch die Verfassung insoweit nicht die Hände gebunden seien (Wallerath).

${ }^{69}$ Vgl. Ch. Starck (Fn. 68) Rn. 36; J. M. Soria (Fn. 68) 647; W. Höfling (Fn. 68) Rn. 31.

70 W. Cremer Freiheitsgrundrechte (Fn. 68) $387 \mathrm{f}$.

${ }^{71}$ Die Hilfen zur Gesundheit nach $\S \S 47$ bis 51 Sozialgesetzbuch (SGB) Zwölftes Buch (XII) - Sozialhilfe - v. 27. 12. 2003, B GB1. I S. 3022, entsprechen genau den Leistungen der gesetzlichen Krankenversicherung. Dies ergibt sich für die Leistungserbringung durch die Krankenkassen aus $\S 264$ Abs. 4 SGB V und für die Leistungserbrin- 
„medizinischen Existenzminimum“. ${ }^{72}$ Nach der engen Auslegung dürfte sich das medizinische Existenzminimum bloß auf gerichtlich noch nicht

gung durch die Sozialhilfeträger aus $§ 52$ SGB XII. Daß der zuständige Sozialhilfeträger die im SGB V vorgesehenen Eigenleistungen übernimmt (so $\S 38$ BSHG in der bis 31. 12. 2003 geltenden Fassung), ist grundsätzlich nicht vorgesehen. Ebenso V. Schlette in: K. Hauck/W. Noftz (Hrsg.) SGB XII, § 52, 2007, Rn. 6 ff., 15 ff. m.w.N.

72 Auch das Recht auf das medizinische Existenzminimum wird zum Teil in Art. 1 Abs. 1 GG iVm dem Sozialstaatsprinzip, zum Teil in Art. 2 Ab. 2 S. 1 GG verortet. Vgl. D. Murswiek (Fn. 68) Rn. 225; Ch. Starck in: H. v. Mangoldt/F. Klein/Ch. Starck (Hrsg.) GG I, 4. Aufl. 1999, Art. 2 Abs. 2, Rn. 212; H. Schulze-Fielitz (Fn. 68) Rn. 96; U. Di Fabio (Fn. 68) Rn. 46, 94; O. Seewald Zum Verfassungsrecht auf Gesundheit, 1981, $70 \mathrm{ff}$.; I. Ebsen Verfassungsrechtliche Implikationen der Ressourcenknappheit im Gesundheitswesen, NDV 1997, 71; P. Kirchhof Gerechte Verteilung medizinischer Leistungen im Rahmen des Finanzierbaren, MMW 1998, 200; V. Neumann Der Grundrechtsschutz von Sozialleistungen in Zeiten der Finanznot, NZS 1998, 401; E. Schmidt-Aßmann Grundrechtspositionen und Legitimationsfragen im öffentlichen Gesundheitswesen, 2001, 23 ff.; M. Nettesheim Rationierung in der Gesundheitsversorgung - verfassungsrechtliche Möglichkeiten und Grenzen, VerwArch 93 (2002) 315; A. Hänlein Festlegung der Grenzen der Leistungspflicht der Krankenkassen, SGb 2003, 301; V. Neumann Das medizinische Existenzminimum, NZS 2006, 393. Das BVerfG argumentiert aus der Perspektive der Versicherten mit einer grundrechtlichen Schutzpflicht aus Art. 2 Abs. 1 GG iVm dem Sozialstaatsgebot bzw. Art. 2 Abs. 2 S. 1 GG (BVerfGE 115, 25 [41 f., 44]; vgl. davor auch schon BVerfG, NJW 1997, 3085 - Edelfosin; BVerfG, NJW 1998, 1775 - Behandlung durch Heilpraktiker; BVerfG, NJW 2004, 3100 - Blutreinigung). Die Lehre geht dagegen zu Recht durchweg von einem originären Leistungsanspruch aus. Inhaltlich wird das medizinische Existenzminimum überwiegend eng definiert (z.B. Di Fabio, Kirchhof, Murswiek, Schulze-Fielitz, Starck). Nur verschiedentlich wird der Standard höher gesetzt: Ebsen (ebd. 78) etwa meint unter Berufung auf Art. 1 Abs. 1 und Art. 20 Abs. 1 GG, daß das medizinische Existenzminimum am „Normalstandard“ für jedermann auszurichten sei - und er hat dabei sichtlich den Leistungskatalog des SGB V aus der Mitte der 1990er Jahre vor Augen (ihm folgend Neumann, Schmidt-Aßmann). Begründend verweist Ebsen darauf, daß das Sozialstaatsprinzip soziale Gleichheit verlange (und daher ein schlechterer Leistungskatalog für Sozialhilfeempfänger abzulehnen sei) und daß die Menschenwürdegarantie insb. auch Stigmatisierungen verbiete: Es dürfe nicht sein, daß man Empfänger von Sozialhilfe an ihren Zähnen erkenne. Für Neumann folgt die Unzulässigkeit der Differenzierung auch daraus, daß der Leistungskatalog des SGB V ohnehin nur das medizinisch Notwendige zusichere; dem Gesetzgeber bleibe deshalb fast kein Spielraum für Leistungsabsenkungen gegenüber Sozialhilfeempfängern. Überzeugend sind die Argumente für die Gleichbehandlung von Versicherten und Empfängern von Sozialhilfe nicht. Zum einen: $\mathrm{Daß}$ die Leistungen der Krankenversicherung grundsätzlich medizinisch notwendig sein müssen, sagt nichts über den Standard aus, an dem diese Notwendigkeit zu messen ist. Zum anderen: Die Aussage, daß das Sozialstaatsprinzip soziale Gleichheit fordere, überdehnt den Inhalt des Sozialstaatsprinzips; die Aussage, daß das Vorenthalten von Leistungen - z.B. des Zahnersatzes - Menschen unzulässig stigmatisiere, überdehnt die Menschenwürdegarantie des Art. 1 Abs. 1 GG (so auch M. Nettesheim ebd. 335, 337). Schließlich: Wenn der medizinische Leistungskatalog des Sozialhilferechts 
definierte „Kernleistungen“ des Krankenversicherungsrechts beziehen. Außerhalb der Kernleistungen des Krankenversicherungsrechts dürften etwa medizinisch empfehlenswerte Behandlungen liegen, aufschiebbare Behandlungen, der Zahnersatz, Kuren, präventive Leistungen oder Maßnahmen der medizinischen Rehabilitation.

Dies führt mich zu meiner dritten These: Das Unterschreiten des Existenzminimums ist grundrechtlich problematisch. Diese Form von sozialer Ungleichheit ist nicht hinzunehmen. Es zeichnet sich allerdings ein breiter Konsens dahin ab, den Mindeststandard deutlich unterhalb der Standards des BSHG anzusetzen. Unter Art. 1 Abs. 1 GG bezieht er sich danach primär auf die Dimensionen Nahrung, Kleidung, Obdach, unter Art. 2 Abs. 2 S. 1 GG auf eine Grundversorgung im Bereich der Gesundheit. Damit nähert sich der Standard des GG dem völkerrechtlichen Standard des Art. 11 des Sozialpakts der Vereinten Nationen. ${ }^{73}$

\section{Alterssicherung und Ungleichheit}

Die verfassungsrechtliche Kritik an der Absenkung des Niveaus der gesetzlichen Rente kann einerseits am Eigentumsrecht, andererseits an Art. 2 Abs. 1 GG ansetzen: Versicherte, die bereits Anwartschaften aus der gesetzlichen Rentenversicherung erworben haben, können sich auf Art. 14 Abs. 1 GG berufen. ${ }^{74}$ Menschen, die ihren Versichertenstatus

dem Katalog des SGB V entspricht und die Versicherten - anders als Sozialhilfeempfänger oder Empfänger von Arbeitslosengeld II - zunehmend zu Eigenleistungen verpflichtet werden, entwickeln sich die Leistungskataloge in die andere Richtung auseinander: Hilfeempfänger erhalten „mehr“ als die Versicherten. Dies ist nicht nur ein politisches Problem, sondern mit Blick auf die Beschäftigten im unteren Lohnbereich wohl auch ein Gleichheitsproblem. Wenn ihr Einkommen nur wenig über den anzuerkennenden Bedarfen des SGB II oder des SGB XII liegt, könnten die Beschäftigten nämlich einwenden, daß sie sich wirtschaftlich in einer vergleichbaren Lage befänden, die Leistungen für sie aber an eine Beitragspflicht gebunden seien. Zu Recht kritisch auch St. Huster (Fn. 67) 161.

${ }^{73}$ International Covenant on Economic, Social and Cultural Rights, General Assembly Resolution 2200 (XXI). Art. 11 Abs. 1 lautet: „The States Parties to the present Covenant recognize the right of everyone to an adequate standard of living for himself and his family, including adequate food, clothing and housing, and to the continuous improvement of living conditions. The States Parties will take appropriate steps to ensure the realization of this right, recognizing to this effect the essential importance of international co-operation based on free consent."

${ }^{74}$ Die Anpassung des aktuellen Rentenwerts genießt den Schutz aus Art. 14 Abs. 1 GG. Eingehend dazu P. Blömeke (Fn. 28) $228 \mathrm{ff}$., der sich auch mit der uneinheitlichen Literatur befaßt. Vgl. weiter BVerfG, Nichtannahmebeschluß v. 26. 7. 2007, 1 BvR $824 / 03$, Rn. 50 = DVB1. 2007, 1228 (1230), wo immer noch offen bleibt, ob die regel- 
nach Inkrafttreten der Rentenreform 2001 erlangt haben, können mit dem Äquivalenzprinzip argumentieren. In beiden Fällen bleiben die Argumente schwach.

Der Schutz aus Art. 14 Abs. 1 GG führt deshalb nicht weit, weil das BVerfG jede Schlechterstellung als Inhalts- und Schrankenbestimmung deutet, und bei der Verhältnismäßigkeitsprüfung dem Gedanken der Finanzierbarkeit der Leistungen ein überragendes Gewicht beimisst. ${ }^{75}$ So hat das BVerfG erhebliche Leistungssenkungen als unproblematisch hingenommen. ${ }^{76}$ Dass der Gesetzgeber mit seinen Maßnahmen ab 2001 diesen Spielraum überschritten hat, ist nicht zu sehen. ${ }^{77}$ Der Anspruch auf „adäquate Versicherungsleistungen“ im Versicherungsfall führt zu nichts, so lange Rentenexperten plausibel machen können, dass die gesetzlichen Renten weiterhin Renditen abwerfen. ${ }^{78}$

Meine vierte These lautet: Aus Art. 14 Abs. 1 oder Art. 2 Abs. 1 GG folgen keine schlagkräftigen Einwände gegen eine Gestaltung der Alterssicherung, die zu größeren Einkommensunterschieden führt. Die Erzeugung von sozialer Ungleichheit ist insoweit unproblematisch.

mäßige Anpassung des aktuellen Rentenwerts unter den Schutz der Eigentumsgarantie fällt.

${ }^{75}$ Näher U. Davy Pfadabhängigkeit in der sozialen Sicherheit, in: Sozialrechtsgeltung in der Zeit, SDSRV 55 (2007) 103 (123).

${ }^{76}$ BVerfGE 58, 81 (109ff.: Neubewertung von Ausbildungszeiten, Minderung der Rente um bis zu 15\%); BVerfGE 64, 87 (97ff.: Abflachung der Rentenanpassung, Minderung der Rente um 13 \%); BVerfGE 70, 101 (110ff.: Änderung der Regeln über Ersatzund Ausfallzeiten, Kürzung der Rente um 25\%); BVerfGE 116, 96 (126ff.: Einführung des Multiplikators von 0,6 im Fremdrentenrecht, Minderung der Renten um bis zu $30 \%$ ); BVerfG, Beschl. v. 27. 2. 2007, 1 BvL 10/00 (Neubewertung der ersten Berufsjahre, Minderung der Rente um $12 \%$ ).

${ }_{77}$ Zur Rentenanpassung nach Maßgabe der Inflationsrate im Jahr 2000 und zur Aussetzung der Anpassung im Jahr 2004 vgl. bereits BSG, Urteil v. 31. 7. 2002, B 4 RA $120 / 00$ R, BSG, Urteil v. 27. 3. 2007, B 13 R 37/06 R, sowie BVerfG, Nichtannahmebeschluß v. 26. 7. 2007, 1 BvR 824/03 = DVB1. 2007, 1228.

${ }^{78}$ Nach den Annahmen der Rentenexperten werden gesetzliche Renten auch nach der Abflachung des Rentenniveaus auf $43 \%$ eine Rendite abwerfen. Vgl. etwa F. Ruland Alterssicherung (Fn. 29) 4, der für Männer, die im Jahr 2030 in Rente gehen, eine Rendite von 2,6\% (nominal) berechnet. Renditeberechnungen beruhen allerdings regelmäBig auf Annahmen, die das Ergebnis „positiv“ beeinflussen. Grundsätzlich ist dabei die Summe der Einzahlungen (aufgezinst) mit der Summe der erwarteten Auszahlungen (abgezinst) zu vergleichen. Da die gesetzliche Rentenversicherung aber nicht nur Altersrenten finanziert (sondern z.B. auch Erwerbsminderungsrenten), werden nicht $100 \%$ der Einzahlungen berücksichtigt, sondern nur $80 \%$. Den Berechnungen liegt auBerdem ein Einzahlungszeitraum von 45 Jahren zugrunde, der in der Praxis kaum erreicht wird. Und die errechneten Renditen sind nicht inflationsbereinigt. Zu den prekären Annahmen solcher Rendite-Berechnungen vgl. näher auch das Gutachten des Sozialbeirats zum Rentenversicherungsbericht 2004 BT-Drs. 15/4498, $89 \mathrm{ff}$. 


\section{Grundsicherung und Ungleichheit}

Die Streitfälle im Bereich der Grundsicherung betreffen einen besonderen Aspekt der Menschenwürdegarantie: Sind die Leistungen des SGB II in ausreichendem Maß bedarfsdeckend? Kommt es zu rechtlich unerträglicher sozialer Ungleichheit?

\section{a) Streit um Bedarfe}

Die ersten Streitfälle betrafen Sozialhilfeempfänger, die schon zu Jahresbeginn die Belastungsgrenze ihrer Zuzahlungspflichten nach dem SGB V ausgeschöpft hatten. ${ }^{79}$ Sollten sie wirklich verpflichtet sein, schon im Januar ihre vollen Eigenanteile aus dem Regelsatz zu bestreiten? Die Sozialgerichte entschärften das Problem mit dem Hinweis auf die Möglichkeit der Darlehensgewährung, die sicherstellt, dass die monatliche Belastung 3,51 bzw. 7,02 Euro nicht überschreitet. ${ }^{80}$ Dann ging es um Bedarfe von jungen Müttern oder Eltern: ${ }^{81}$ Konnte es wirklich sein, dass im Fall der Geburt alle Baby-Bedarfe mit einem Schlag aus der Regelleistung zu bestreiten waren? Einige Gerichte akzeptierten waghalsig auch nach SGB II einen Anspruch auf einmalige Leistungen. ${ }^{82}$ Später wurde über die Möglichkeit gestritten, Empfängern von Arbeitslosengeld II eine Haushaltshilfe zu gewähren, ${ }^{83}$ die Aufwendungen für

${ }^{79}$ VG Berlin, Beschl. v. 2. 4. 2004, 8 A $69.04=\mathrm{ZFSH} / \mathrm{SGB}$ 2005, 278; Nds. OVG, Beschl. v. 6. 5. 2004, $4 \mathrm{ME} 88 / 04$. Zur Gewährung einer Beihilfe für die Neuanschaffung einer Brille weiter Nds. OVG, Beschl. v. 13. 8. 2004, 4 ME 224/04 = ZFSH/SGB 2004, 560; Bayer. VGH München, Beschl. v. 2. 9. 2004, 12 CE $04.979=$ ZFSH/SGB 2004, 612; Hess. VGH, Beschl. v. 22. 11. 2004, 10 TG 3128/04 = ZFSH/SGB 2005, 547.

${ }^{80}$ Für die Rechtslage unter dem SGB XII zustimmend BSG, Urteil v. 22. 4. 2008, B 1 KR 10/07 R, Rn. 30. Für die Rechtslage unter dem BSHG war die Gesetzmäßigkeit der Darlehenslösung strittig. Vgl. näher N. Schumacher Das Dilemma der Rechtsprechung zur Kostenübernahme von Gesundheitsleistungen durch die Sozialhilfe, RdL 2004, 123; P. Armbrust Urteilsanmerkung, ZFSH/SGB 2004, 549.

${ }^{81}$ SG Hamburg, Beschl. v. 23. 3. 2005, S 57 AS 125/05 ER; SG Speyer, Beschl. v. 13. 6. 2005, S 16 ER 100/05 AS; LSG Rh-Pf., Beschl. v. 12. 7. 2005, L 3 ER 45/05 AS = FEVS 57, 181; SG Berlin, Beschl. v. 20. 2. 2006, S 104 AS 1172/06 ER; LSG BerlinBrandenburg, Beschl. v. 3. 3. 2006, L 10 B 106/06 AS ER; LSG Nds.-Bremen, Beschl. v. 16. 5. 2006, L 6 AS 170/06 ER.

82 Vgl. z. B. LSG Rh-Pf., Beschl. v. 12. 7. 2005, L 3 ER 45/05 AS, Rn. 14 (Subsumtion unter „Erstausstattung der Wohnung“; Tragetuch mit Blick auf die soziokulturellen Gegebenheiten in Deutschland nicht zumutbar). Dann sprang der Gesetzgeber ein. Vgl. $\S 23$ Abs. 3 S. 1 Nr. 2 SGB II idF des Gesetzes zur Fortentwicklung der Grundsicherung für Arbeitsuchende v. 20. 7. 2006, BGBl. I S. 1706 (Art. 1 Nr. 22).

${ }_{83}$ Dies war nach $\S 11$ Abs. 3 BSHG in vielen Fällen unproblematisch zulässig. Einen Anspruch für Arbeitslosengeld II-Empfänger mangels Rechtsgrundlage ablehnend SG Hamburg, Beschl. v. 13. 6. 2005, S 51 SO 267/05 ER. Einen Anspruch auf der Grund- 
Hygienebedarfe oder Arzneimittel abzufangen, ${ }^{84}$ oder die Kosten für die Ausübung des Umgangsrechts mit Kindern zu decken, wenn die Eltern geschieden sind oder getrennt leben. ${ }^{85}$ Nach früherem Recht wäre eine Erhöhung des Regelsatzes in Betracht gekommen, das SGB II schließt dies aus. Interessant ist freilich, dass die Sozialgerichte - bei aller Bereitschaft zur kleinen Korrektur - bislang nicht versucht waren, die Angemessenheit der Regelleistung selbst in Frage zu stellen. ${ }^{86}$

lage von $\S 70$ Abs. 1 SGB XII bejahend LSG Hamburg, Beschl. v. 21. 4. 2005, L 3 B 70/05 ER SO; LSG Hamburg, Beschl. v. 5. 7. 2005, L 5 B 159/05 ER AS; LSG NRW, Beschl. v. 16. 9. 2005, L 20 B 9/05 SO ER. Einen Anspruch auf der Grundlage von $§ 61$ Abs. 1 S. 2 SGB XII bejahend: LSG Nds.-Bremen, Beschl. v. 24. 8. 2005, L 8 SO 78/05 ER; LSG BW, Beschl. v. 7. 3. 2006, L 7 SO 509/06 ER-B; LSG Berlin-Brandenburg, Beschl. v. 30. 6. 2006, L 15 B 45/06 SO ER; Hess. LSG, Beschl. v. 4. 7. 2006, L 9 SO 24/06 ER. Der letztgenannten Auffassung hat sich das BSG, gestützt auf eine verfassungskonforme Interpretation, angeschlossen (BSG, Urteil v. 11. 12. 2007, B 8/9b SO 12/06 R). Vgl. ferner S. Knickrehm Haushaltshilfe für Empfänger von Arbeitslosengeld II?, NZS 2007, 128; B. Schütze Rechtsprechung zu Mehr- und Sonderbedarfen in besonderen Not- und Sondersituationen, SozSich 2007, 113.

84 Vgl. z.B. SG Köln, Beschl. v. 5. 2. 2007, S 6 AS 4/07 ER; LSG Berlin-Brandenburg, Beschl. v. 24. 4. 2007, L 19 B 400/07 AS ER; LSG BW, Urteil v. 22. 11. 2007, L 7 SO 4180/06; LSG NRW, Beschl. v. 21. 12. 2007, L 19 B 134/07 AS ER; LSG NRW, Beschl. v. 7. 2. 2008, L 7 B 313/07 AS.

85 Vgl. zunächst BSG, Urteil v. 7. 11. 2006, B 7b AS 14/06 R = NZS 2007, 383, und danach: LSG Berlin-Brandenburg, Beschl. v. 21. 11. 2006, L 10 B 1061/06 AS ER; LSG Berlin-Brandenburg, Beschl. v. 19. 12. 2006, L 10 B 1179/06 AS PKH; LSG NRW, Beschl. v. 10. 5. 2007, L 20 B 24/07 SO ER; LSG Nds.-Bremen, Urteil v. 21. 6. 2007, L 8 AS 491/05; SG Dortmund, Urteil v. 23. 8. 2007, S 22 AS 17/06; LSG NRW, Urteil v. 6. 9. 2007, L 9 AS 80/06; LSG NRW, Urteil v. 21. 4. 2008, L 20 AS 112/06. Vgl. ferner S. Knickrehm Kosten des Umgangsrechts und Regelleistungen nach dem SGB II, Sozialrecht aktuell 2006, 159; U. Wenner Erste BSG-Urteile zu Hartz IV stärken teilweise Rechte der Betroffenen, SozSich 2006, 391; W. Schellhorn Sozialrechtliche Leistungen zur Ermöglichung des Umgangsrechts, FuR 2007, 193; M. Gerenkamp/J. Kroker Ergänzende Sozialhilfeleistungen für Leistungsempfänger nach dem SGB II am Beispiel des elterlichen Umgangsrechts, NZS 2008, 28; K. Krauß in: K. Hauck/W. Noftz (Hrsg.) SGB II, 2008, § 20 Rn. 19f. Zur verfassungsrechtlichen Dimension des Umgangsrechts BVerfG, Beschl. v. 25. 10. 1994, 1 BvR 1197/93 = NJW 1995, 1342; BVerfG, Urteil v. 1. 4. 2008, 1 BvR 1620/04 = NJW 2008, 1287.

${ }^{86}$ Das BSG und viele LSG bestätigten die Verfassungskonformität von $\S 20$ Abs. 2 SGB II. In zeitlicher Reihenfolge: LSG BW, Urteil v. 2. 9. 2005, L 8 AS 1995/05; Bayer. LSG, Urteil v. 18. 5. 2006, L 11 AS 111/05; LSG Nds.-Bremen, Urteil v. 24. 8. 2006, L 8 AS 467/05; Sächs. LSG, Urteil v. 19. 10. 2006, L 3 AS 5/05; LSG Berlin-Brandenburg, Beschl. v. 20. 11. 2006, L 10 B 1014/05 AS PKH; BSG, Urteil v. 23. 11. 2006, B 11b AS 1/06 R = FEVS 58, 353; LSG NRW, Urteil v. 8. 11. 2007, L 9 AS 62/06; BSG, Beschl. v. 27. 2. 2008, B 14 AS 160/07 B; vgl. aber auch Sächs. LSG, Urteil v. 29. 3. 2007, L 3 AS 101/06 (Kosten für Warmwasseraufbereitung nicht Teil der Regelleistung). Kritisch dagegen P. Mrozynski Grundsicherung für Arbeitsuchende, im Alter, bei voller 
Vor diesem Hintergrund formuliere ich meine fünfte These: Das SGB II hat den Menschenwürde-Standard des BSHG deutlich abgesenkt. Die Sozialgerichte sind verunsichert und bemüht, dem durch verfassungskonforme Auslegung gegenzusteuern. Sie scheuen aber davor zurück, mit dem Gesetzgeber in einem Gerichtsverfahren über den genauen Verlauf der denkbar untersten Grenze des Existenzminimums zu streiten.

\section{b) Multiple Exklusion}

Aus dogmatischer Sicht unbefriedigend sind nicht nur die unhaltbaren Versuche, für manche Bedarfe doch eine Rechtsgrundlage im SGB II oder SGB XII zu finden. Problematisch ist auch, dass der gerichtliche Rechtsschutz insgesamt hinter den Anforderungen des Art. 1 Abs. 1 GG zurückbleibt.

Die Sozialpolitik der letzten fünf Jahre konfrontiert uns mit einem neuen Phänomen, nämlich der Exklusion von Bedürfnissen aus mehreren Funktionssystemen. Das ist nicht die Vollexklusion, die die Soziologie beschäftigt, ${ }^{87}$ aber es sind spürbare Teilexklusionen, die soziale Ungleichheit verschärfen: Die Ausgrenzungen im Bereich der gesetzlichen Krankenversicherung werden weder durch die Sozialhilfe noch durch die Grundsicherung aufgefangen. Einzelne Bedarfe des Lebensunterhalts, die noch vor fünf Jahren durch Öffnungsklauseln des Sozialhilferechts gedeckt waren, sind heute aus der Regelleistung zu bestreiten. Freilich, die Rücknahme von Leistungen im Grenzbereich der Menschenwürde ist nicht schon grundsätzlich problematisch. Wenn die einfachgesetzlichen Garantien großzügiger sind als das verfassungsrecht-

Erwerbsminderung und die Sozialhilfereform, ZFSH/SGB 2004, 198; W. Däubler Das Verbot der Ausgrenzung einzelner Bevölkerungsgruppen - Existenzminimum und Arbeitslosengeld II, NZS 2005, 225; K.-J. Bieback Probleme des SGB II, NZS 2005, 337; E. Ockenga Regelleistung des SGB II und Verfassungswidrigkeit, ZFSH/SGB 2006, 143; J. Münder Das Leistungsrecht des SGB II: Erfahrungen mit pauschalierten Leistungen, NZS 2008, 169; T. Hebeler Die verfassungsrechtliche Einordnung der pauschalierenden Leistungsgestaltung im SGB II, SGb 2008, 8; K. Krauß (Fn. 85) Rn. 17 f. Differenzierend $W$. Spellbrink Gelingt durch die neuen Instrumente des SGB II die Integration der Langzeitarbeitslosen in den Arbeitsmarkt?, SGb 2008, 445 (451).

${ }^{87}$ R. Stichweh (Fn. 14); L. Leisering (Fn. 39); L. Leisering „Soziale Ausgrenzung“ Zur handlungstheoretischen Fundierung eines aktuellen sozialpolitischen Diskurses, in: St. Hradil (Hrsg.) Differenz und Integration. Die Zukunft moderner Gesellschaften. Verhandlungen des 28. Kongresses der Deutschen Gesellschaft für Soziologie in Dresden 1996, 1997, 1040; M. Kronauer Exklusion. Die Gefährdung des Sozialen im hoch entwickelten Kapitalismus, 2003, ferner die Beiträge in: H. Bude/A. Willisch (Hrsg.) Das Problem der Exklusion. Ausgegrenzte, Entbehrliche, Überflüssige, 2006; H. Bude/A. Willisch (Hrsg.) Exklusion. Die Debatte der „Überflüssigen“, 2007. 
lich Geforderte, ist Leistungsabbau unproblematisch. Problematisch ist, dass das Rechtsschutzsystem nicht garantiert, dass die denkbar unterste Grenze des Existenzminimums beachtet wird. Aus Gründen der $\mathrm{Zu}-$ ständigkeit, aber auch weil Bedarfe nicht zeitgleich entstehen, sind der Ausschluss der Sehhilfen aus dem SGB V im Verfahren A, der besondere Hygienebedarf im Verfahren B, die Kosten des Umgangsrechts im Verfahren $\mathrm{C}$ und die Notwendigkeit eines Ersatzkühlschranks im Verfahren D geltend zu machen, zum Teil vor verschiedenen Leistungsträgern. So gerät das, was die Armutsforschung „deprivation“ nennt, in seiner Gesamtheit nicht in den Blick. Im Verfahren über eine Verfassungsbeschwerde ist es schwierig, die Beschwerde umfassend darzutun. ${ }^{88}$ Ein Vorlageverfahren gegen die Höhe der Regelleistung scheitert an der mangelnden Bereitschaft der Gerichte, sich allgemein und abstrakt mit der Angemessenheit des Geldbetrages auseinanderzusetzen. ${ }^{89}$

Meine sechste These ist daher: Die Sozialpolitik der letzten Jahre führt gerade für Menschen, die Arbeitslosengeld II oder Sozialhilfe beziehen, zu multipler Exklusion. Es gibt aber kein Gerichtsverfahren, in dem diese spezifische Form der Exklusion angemessen thematisiert werden könnte. So gibt es keine Instanz, die die Einhaltung der Menschenwürde-Garantie verlässlich prüfen kann.

\footnotetext{
${ }^{88}$ Vgl. BVerfG, Nichtannahmebeschluß v. 7. 11. 2007, 1 BvR 1840/07, und dazu K.-J. Bieback Urteilsanmerkung, SGb 2008, 409 (410); U. Wenner Bundesverfassungsgericht zur Höhe der ALG-II-Regelsätze: Fast alle Fragen offen, SozSich 2008, 36.

${ }^{89}$ Mit Blick auf die universelle Tauschkraft von Geld scheint naheliegend, den Vorwurf der fehlenden Existenzsicherung auf die Regelleistung nach $§ 20$ Abs. 2 SGB II zu konzentrieren: Wäre die Regelleistung höher, wäre die Inflexibilität der Geldleistungen nicht so spürbar und gewisse zusätzliche Aufwendungen (für die Gesundheit, das Umgangsrecht oder größere Anschaffungen im Haushalt) könnten leichter finanziert werden. So könnte man argumentieren. Drei Umstände machen ein Vorlageverfahren nach Art. 100 Abs. 1 GG dennoch schwierig: Erstens muß sich der Angriff formal ausschließlich gegen § 20 Abs. 2 SGB II richten, obwohl der Gesetzgeber die Unterschreitung des Existenzminimums auch durch Änderung eines anderen Gesetzes beheben könnte (z.B. durch Änderung des SGB V). Zweitens schafft die „Segmentierung“ der Exklusion Wahrnehmungsbarrieren. Dies macht ein Vorlageverfahren faktisch unwahrscheinlich. Eine dritte Hürde liegt in der beträchtlichen Darlegungslast, die das BVerfG den Gerichten auferlegt.
} 


\section{Europäisierung der Gleichheit}

\section{Angleichung der Lebens- und Arbeitsbedingungen}

Der Gründungsvertrag der EWG fasste soziale Ungleichheit zum ersten Mal „europäisch“. Die Angleichung der Lebens- und Arbeitsbedingungen zwischen den Mitgliedstaaten gehörte von Anfang an zu den Zielen der Gemeinschaft. ${ }^{90}$ Für die 15 westeuropäischen Mitgliedstaaten - nur soweit machen Aussagen Sinn - haben sich die Relationen in der Tat verändert. Die Wirtschaften sind expandiert, die Wohlstandsunterschiede zwischen und in vielen Mitgliedstaaten sind kleiner geworden (vgl. Anhang, Tabelle 1). ${ }^{91}$

Ich will den Ursachen für diesen Erfolg nicht nachgehen. Mich interessiert der Einfluß des Gemeinschaftsrechts aus der Perspektive der Arbeitskräfte: Welchen Beitrag zur sozialen Positionierung leistet das Gemeinschaftsrecht für jene Menschen und ihre Nachkommen, die von ihrer Freizügigkeit Gebrauch machen? Die Frage hat für Deutschland einen besonderen Reiz. Die ersten Gastarbeitergenerationen kamen vor allem auch aus Italien, Griechenland, Spanien und Portugal. Die Zuwanderung erfolgte zunächst nicht auf der Grundlage des Gemeinschaftsrechts, sondern auf der Grundlage von Anwerbeabkommen. Die Anwerbung zielte bewusst auf Nicht- bzw. Geringqualifizierte, und die Einwanderung erfolgte stets von einem deutlich ärmeren Staat in einen deutlich reicheren Staat. ${ }^{92}$ Insoweit unterscheidet sich die frühe Zuwan-

${ }_{90}$ Ursprünglich Art. 117 Abs. 1 EWGV, heute Art. 2 und Art. 136 Abs. 1 EGV.

91 Der Wohlstand - gemessen in GNI per capita - hat sich in den vergangenen Jahrzehnten in allen Mitgliedstaaten vervielfacht. Für Irland etwa sind die Werte zwischen 1975 und 2005 um den Faktor 13 gewachsen. Bemerkenswert ist, daß sich die Abstände zwischen dem reichsten und dem ärmsten Mitgliedstaat in der Gruppe der EU15 in den letzten 20 Jahren verkleinert haben. Im Jahr 1985 war Luxemburg der reichste, und Portugal der ärmste Mitgliedstaat. Der Wohlstandswert für Luxemburg war um das 5,14-fache größer als der Wohlstandswert für Portugal. Im Jahr 2005 - nach wie vor war Luxemburg der reichste und Portugal der ärmste Mitgliedstaat - war die Wohlstandsdifferenz zwischen den beiden Staaten auf das 3,79-fache gesunken (s. Anhang, Tabelle 1). Die Gini-Koeffizienten - sie messen die Einkommensungleichheit in den einzelnen Mitgliedstaaten - sanken bis Mitte der 1990er Jahre in Dänemark, Griechenland, Finnland, Schweden sowie Spanien und ab 1995 in Frankreich und Irland. Zu einer Vergrößerung der Einkommensunterschiede kam es zwischen 1975 und 1985 v.a. im Vereinigten Königreich, zwischen 1985 und 1995 in Italien, und zwischen 1995 und 2005 in Finnland und in Schweden. Vgl. dazu im einzelnen European Commission The Social Situation in the European Union 2007. Social Cohesion through Equal Opportunities, 2008, $12 \mathrm{ff}$.

92 Aus historischer Perspektive K. J. Bade Einheimische Ausländer: ,Gastarbeiter ‘ Dauergäste - Einwanderer, in: K. J. Bade (Hrsg.) Deutsche im Ausland - Fremde in 
derung aus späteren EU-Mitgliedstaaten nicht von der Zuwanderung aus Jugoslawien oder der Türkei, um die beiden anderen großen Anwerbeländer zu nennen. ${ }^{93}$ Schlägt sich der Schutz durch das Gemeinschaftsrecht für Angehörige der EU-Anwerbeländer im sozialen Status nieder?

Deutschland. Migration in Geschichte und Gegenwart, 1992, $393 \mathrm{ff}$.; aus ökonomischer Perspektive G. Schiller Die Bedeutung der Ausländerbeschäftigung für die Volkswirtschaft, in: K. J. Bade (Hrsg.) Auswanderer Wanderarbeiter Gastarbeiter. Bevölkerung, Arbeitsmarkt und Wanderung in Deutschland seit der Mitte des 19. Jahrhunderts, Bd. 2, 2. Aufl. 1985, $625 \mathrm{ff}$.

${ }^{93}$ Die Angehörigen der genannten Anwerbeländer bildeten und bilden den weitaus größten Teil der ausländischen Wohnbevölkerung. Im Jahr 1982 zählten zur ausländischen Wohnbevölkerung 4,66 Millionen Menschen, darunter waren 106005 Einwanderer aus Portugal, 173526 aus Spanien, 300824 aus Griechenland, 601621 aus Italien, 631692 aus Jugoslawien und 1,58 Mio. aus der Türkei (zusammen 72,7\% der ausländischen Wohnbevölkerung). Angaben bei: J. K. Bade Die Ausländerbeschäftigung in der Bundesrepublik zwischen Arbeitswanderung und Einwanderung. Einleitung, in: K. J. Bade (Hrsg.) Auswanderer (Fn. 92) 621 (624). Im Jahr 1996 zählten zur ausländischen Wohnbevölkerung 130842 Einwanderer aus Portugal (1,8\%), 132457 aus Spanien (1,8\%), 362539 aus Griechenland (5,0\%), 599429 aus Italien (8,2\%), 754311 aus der Bundesrepublik Jugoslawien (10,3\%) und 2,05 Mio. aus der Türkei (28,0\%). Angaben aus: Bericht der Beauftragten der Bundesregierung für die Belange der Ausländer über die Lage der Ausländer in der Bundesrepublik Deutschland 1997, BT-Drs. 13/9484, 97. Die Angehörigen der genannten Anwerbeländer bildeten damit noch immer 55\% der ausländischen Wohnbevölkerung. Ab Anfang der 1990er Jahre war bereits die Zuwanderung aus Osteuropa wirksam, insbesondere aus Polen und Rumänien. Im Jahr 2006 umfaßte die ausländische Wohnbevölkerung 115028 Staatsangehörige Portugals (1,7\%), 106819 Staatsangehörige Spaniens (1,6\%), 303761 Staatsangehörige Griechenlands (4,5\%), 534657 Staatsangehörige Italiens (7,9\%), 750075 Staatsangehörige eines der Nachfolgestaaten des früheren Jugoslawien (11,8\%) und 1,74 Mio. Staatsangehörige der Türkei (25,8\%). Angaben aus: 7. Bericht der Beauftragten der Bundesregierung für Migration, Flüchtlinge und Integration über die Lage der Ausländerinnen und Ausländer in Deutschland, 2007, 209. Die innergemeinschaftliche Migration der jüngsten Vergangenheit weist andere Merkmale auf als die Migration im Wirtschaftsboom der Nachkriegszeit und im folgenden wirtschaftlichen Abschwung. Vgl. dazu insb. R. Verwiebe/K. Wunderlich/Ch. Münzing Die Einkommenssituation transnational mobiler Europäer auf dem Berliner Arbeitsmarkt. Zählt nationale Herkunft oder sozialstrukturelle Position?, Zeitschrift für Soziologie 32 (2003) 418; R. Verwiebe/M. C. Müller Gelungene Integration in den Arbeitsmarkt? Die flexiblen Biografien transnational mobiler Europäer zu Beginn des 21. Jahrhunderts, Berliner Journal für Soziologie 2006/1, 95; $R$. Verwiebe Transnationale Mobilität innerhalb Europas und soziale Ungleichheit, in: M. Heidenreich (Hrsg.) Die Europäisierung sozialer Ungleichheit. Zur transnationalen Klassen- und Sozialstrukturanalyse, 2006, 155. 


\section{Unionsbürgerschaft: Recht auf soziale Teilhabe}

Das Gemeinschaftsrecht garantiert Arbeitnehmern aus einem anderen Mitgliedstaat der EU zum einen ein relativ sicheres Aufenthaltsrecht. ${ }^{94}$ Das Gemeinschaftsrecht garantiert zum anderen rechtliche Gleichheit: Arbeitnehmer aus anderen Mitgliedstaaten haben wie Deutsche Zugang zu einer Beschäftigung ihrer Wahl. ${ }^{95}$ Sie haben ein Recht auf gleichen Zugang zu den Systemen der sozialen Sicherheit. ${ }^{96}$ Arbeitnehmer aus anderen Mitgliedstaaten haben nach Art. 7 Abs. 2 VO 1612/68 außerdem ein Recht auf gleiche soziale Vergünstigungen. ${ }^{97}$ Es war von Anfang an klar, dass das Arbeitsgenehmigungsrecht auf Arbeitskräfte aus anderen Mitgliedstaaten keine Anwendung finden durfte. Unproblematisch war auch die Eingliederung der Arbeitskräfte in die Systeme der sozialen Sicherheit. Sozialversicherungsrecht ist - wegen der Anknüpfung an die Beschäftigung - weitgehend inkludierend. Problematisch war die Reichweite des Gleichheitssatzes des Art. 7 Abs. 2 VO 1612/68. Der Gleichheitssatz bezieht sich auf steuerfinanzierte Sozialleistungen, und hier verhalten sich Staaten traditionell exkludierend.

Der EuGH hat entschlossen dafür gesorgt, dass die rechtliche Gleichheit für Arbeitskräfte aus anderen Mitgliedstaaten nicht nur eine beschäftigungsrechtliche, sondern zugleich eine umfassende sozialrechtliche Dimension hat. Anfangs sprach der EuGH bei der Auslegung des Art. 7 Abs. 2 VO 1612/68 wohl noch vage von einem Recht auf alle Vergünstigungen, die die Mobilität innerhalb der Gemeinschaft erleich-

${ }^{94}$ Vgl. Art. 39 Abs. 3 EGV und die Richtlinie 2004/38/EG über das Recht der Unionsbürger und ihrer Familienangehörigen, sich im Hoheitsgebiet der Mitgliedstaaten frei zu bewegen und aufzuhalten, zur Änderung der Verordnung (EWG) Nr. 1612/68 und zur Aufhebung der Richtlinien 64/221/EWG, 68/360/EWG, 72/194/EWG, 73/148/EWG, 75/34/EWG, 75/35/EWG, 90/364/EWG, 90/365/EWG und 93/96/ EWG v. 29. 4. 2004, ABl. Nr. L 158/77.

95 Vgl. Art. 39 Abs. 2 EGV (Abschaffung jeder auf der Staatsangehörigkeit beruhenden unterschiedlichen Behandlung der Arbeitnehmer der Mitgliedstaaten in Bezug auf Beschäftigung, Entlohnung und sonstige Arbeitsbedingungen) und Verordnung (EWG) Nr. 1612/68 über die Freizügigkeit der Arbeitnehmer innerhalb der Gemeinschaft v. 15. 10. 1968 ABl. L 257/2, zuletzt geändert durch die Richtlinie 2004/38/EG.

${ }^{96}$ Art. 3 der Verordnung (EWG) Nr. 1408/71 zur Anwendung der Systeme der sozialen Sicherheit auf Arbeitnehmer und Selbständige sowie deren Familienangehörige, die innerhalb der Gemeinschaft zu- und abwandern, v. 14. 6. 1971, ABl. Nr. L 149/2, zuletzt geändert durch Verordnung (EG) Nr. 592/2008, ABl. Nr. L 177/1; vgl. auch Art. 4 der Verordnung (EG) Nr. 883/2004 zur Koordinierung der Systeme der sozialen Sicherheit v. 29. 4. 2004, ABl. Nr. L 166/1.

${ }^{97}$ Art. 7 Abs. 2 VO 1612/68 lautet: „[Ein Arbeitnehmer, der Staatsangehöriger eines Mitgliedstaats ist,] genießt [im Hoheitsgebiet der anderen Mitgliedstaaten] die gleichen sozialen und steuerlichen Vergünstigungen wie die inländischen Arbeitnehmer“. 
terten. ${ }^{98}$ Seit Mitte der 1980er Jahre orientiert sich der EuGH an einer Begründungserwägung der Verordnung, die soziale Ungleichheit ausdrücklich anspricht: Arbeitskräfte sollen damit nämlich insbesondere die Möglichkeit zum sozialen Aufstieg erhalten. Daran anknüpfend ${ }^{99}$ deutet der EuGH praktisch jede staatliche Maßnahme, die sozial Schwache fördert, als eine soziale Vergünstigung im Sinne des Art. 7 Abs. 2 VO 1612/68. Das gilt für bedarfsunabhängige Sozialleistungen wie das Kindergeld, das Erziehungsgeld oder das Elterngeld. ${ }^{100}$ Es gilt für bedarfsabhängige Sozialleistungen wie die Grundsicherung oder die Sozialhilfe. ${ }^{101}$ Und es gilt für alle Leistungen der Ausbildungsförderung. ${ }^{102}$ Träger dieser Rechte sind nicht nur die Arbeitnehmer selbst, sondern auch die Ehepartner und Familienangehörige in auf- und absteigender Linie, solange ihnen Unterhalt gewährt wird.103 In den Händen des EuGH ist das Gleichheitsrecht außerdem eine scharfe Waffe. Das Recht kann nicht durch andere Überlegungen kompromittiert werden. ${ }^{104}$ Dass

98 EuGH, Urteil v. 31. 5. 1979, Even, EuSlg. 1979, 2019, Rn. 22; EuGH, Urteil v. 14. 1. 1982, Reina, EuSlg. 1982, 33, Rn. 12.

99 Vgl. zunächst EuGH, Urteil v. 11. 7. 1985, Mutsch, EuSlg. 1985, 2681, Rn. 15, später EuGH, Urteil v. 21. 6. 1988, Lair, EuSlg. 1988, 3161, Rn. 20; EuGH, Urteil v. 27. 9. 1988, Matteucci, EuSlg. 1988, 5589, Rn. 11.

100 Z.B. EuGH, Urteil v. 18. 7. 2007, Hartmann, EuSlg. 2007 I 6303 (Erziehungsgeld).

${ }^{101}$ EuGH, Urteil v. 12. 7. 1984, Castelli, EuSlg. 1984, 3199 (garantiertes Altersmindesteinkommen); EuGH, Urteil v. 6. 6. 1985, Frascogna, EuSlg. 1985, 1739 (allocation spéciale de vieillesse); EuGH, Urteil v. 9. 7. 1987, Frascogna, EuSlg. 1987, 3431 (allocation spéciale de vieillesse); EuGH, Urteil v. 27. 3. 1985, Hoeckx, EuSlg. 1985, 973 (Hilfe zum Lebensunterhalt); EuGH, Urteil v. 27. 3. 1985, Scrivner, EuSlg. 1985, 1027 (Hilfe zum Lebensunterhalt); EuGH, Urteil v. 20. 6. 1985, Deak, EuSlg. 1985, 1873 (Überbrückungsgeld für junge Arbeitslose); EuGH, Urteil v. 18. 6. 1987, Lebon, EuSlg. 1987, 2811 (Existenzminimum); EuGH, Urteil v. 27. 5. 1993, Hugo Schmid, EuSlg. 1993 I 3011 (Leistung für Behinderte).

102 EuGH, Urteil v. 21. 6. 1988, Lair, EuSlg. 1988, 3161; EuGH, Urteil v. 21. 6. 1988, Brown, EuSlg. 1988, 3205; EuGH, Urteil v. 15. 3. 1989, Echternach, EuSlg. 1989, 723; EuGH, Urteil v. 26. 2. 1992, Bernini, EuSlg. 1992 I 1071.

103 Z.B. EuGH, Urteil v. 12. 7. 1984, Castelli, EuSlg. 1984, 3199 (Mutter eines Arbeitnehmers); EuGH, Urteil v. 26. 2. 1992, Bernini, EuSlg. 1992, 1071 (Tochter eines Arbeitnehmers); EuGH, Urteil v. 18. 7. 2007, Hartmann, EuSlg. 2007, 6303 (Ehefrau eines Arbeitnehmers).

${ }^{104}$ Eine Erklärung für die erstaunliche Schlagkraft des Gleichheitsrechts aus Art. 7 Abs. 2 VO 1612/68 ist, daß die Verfahren bis weit in die 1990er Jahre primär unmittelbare Diskriminierungen betrafen. Hier folgt aus der Feststellung, daß eine Ungleichbehandlung nach Maßgabe der Staatsangehörigkeit vorliegt, ohne weiteres, daß die Ungleichbehandlung unzulässig ist. Aber auch in den jüngeren Fällen, die mittelbare Diskriminierungen betreffen, akzeptiert der EuGH die von den Regierungen vorgetragenen Rechtfertigungen selten. 
der EuGH heute soziale Rechte unmittelbar in der Unionsbürgerschaft verankert, sei angemerkt, aber nicht weiter verfolgt. ${ }^{105}$

Ich formuliere meine siebente These: Aus der Sicht des Gemeinschaftsrechts ist soziale Ungleichheit problematisch, wenn sie ihre Basis in rechtlicher Ungleichheit hat. Die Rechtsprechung des EuGH zu den „sozialen Vergünstigungen“ gibt der Freizügigkeit eine starke sozialrechtliche Komponente. Social citizenship ist Teil der Unionsbürgerschaft. Das hat explizit das Ziel, mehr soziale Gleichheit herzustellen.

\section{Mehr soziale Gleichheit}

Es gibt kaum Untersuchungen, die die Sozialstruktur der Angehörigen der EU-Anwerbeländer (Italien, Griechenland, Spanien, Portugal) mit der Sozialstruktur der Angehörigen der anderen großen Anwerbeländer (Türkei, früheres Jugoslawien) vergleichen. Soweit es sie gibt, deuten sie darauf hin, dass sich die Sozialstruktur unterscheidet. ${ }^{106}$ Die Daten des Sozio-ökonomischen Panels ${ }^{107}$ bekräftigen den Eindruck.

${ }^{105}$ Der EuGH stützt sich insbesondere dann auf die Unionsbürgerschaft in Verbindung mit dem allgemeinen Diskriminierungsverbot aus Art. 12 EGV, wenn die Arbeitnehmereigenschaft der Kläger im Ausgangsverfahren unklar bleibt oder feststeht, daß die Kläger die Arbeitnehmereigenschaft nicht besitzen. Vgl. insb. EuGH, Urteil v. 12. 5. 1998, Sala, EuSlg. 1998 I 2691; EuGH, Urteil v. 20. 9. 2001, Grzelczyk, EuSlg. 2001 I 6193; EuGH, Urteil v. 23. 3. 2004, Collins, EuSlg. 2004 I 2703; EuGH, Urteil v. 7. 9. 2004, Trojani, EuSlg. 2004 I 7573. Die Rechtsprechung ist nicht unumstritten. Vgl. jüngst Ch. Tomuschat Gleichheit in der Europäischen Union, ZaöRV 68/2 (2008) 327 ff., m.w.N.

${ }_{106}$ U. Mehrländer/C. Ascheberg/J. Ueltzhöffer Repräsentativuntersuchung '95: Situation der ausländischen Arbeitnehmer und ihrer Familienangehörigen in der Bundesrepublik Deutschland, 1996; I. Tucci/G. G. Wagner Above-Average Rise in Immigrant Poverty, DIW Berlin, Weekly Report No. 5/2005, 69; A. Uhlendorff/K. $F$. Zimmermann Unemployment Dynamics among Migrants and Natives, DIW Berlin, Discussion Papers No. 617, 2006; 6. Bericht der Beauftragten der Bundesregierung für Migration, Flüchtlinge und Integration über die Lage der Ausländerinnen und Ausländer in Deutschland, 2005, 104; 7. Bericht der Beauftragten der Bundesregierung für Migration, Flüchtlinge und Integration über die Lage der Ausländerinnen und Ausländer in Deutschland, 2007; Statistisches Bundesamt (Hrsg.) Bevölkerung und Erwerbstätigkeit. Bevölkerung mit Migrationshintergrund. Ergebnisse des Mikrozensus 2006, 2008.

107 Das Sozio-ökonomische Panel (SOEP) ist eine jährliche Wiederholungsbefragung privater Haushalte. Die Befragung wird vom Deutschen Institut für Wirtschaftsforschung in Kooperation mit Infratest durchgeführt. Die erste Erhebungswelle fand im Jahr 1984 statt. Das Panel erfaßt nicht nur eine Stichprobe der Haushalte deutscher Staatsangehöriger, sondern auch eine Stichprobe der ausländischen Haushalte. 
Die Ergebnisse der Erhebungswelle 1986 spiegeln noch immer wider, dass die Einwanderung aus den Anwerbeländern auf Beschäftigung und Geringqualifizierte zielte: ${ }^{108} 30$ bis $40 \%$ der Befragten aus den Anwerbeländern geben 1986 an, über keinen Schulabschluss zu verfügen, zwischen 50 und $70 \%$ verfügten über keinen Berufsabschluss. ${ }^{109}$ Der Anteil der Nicht-Erwerbstätigen ist viel kleiner als bei den Deutschen. ${ }^{110}$ In diesen frühen Daten heben sich die Angehörigen der EU-Anwerbeländer nicht positiv von den Einwanderern aus Jugoslawien oder der Türkei ab, weder in der Qualifikation noch bei den Erwerbseinkommen (Anhang, Tabelle 4). ${ }^{111}$ Im Trend der Erhebungswellen 1996 und 2006 sind wichtige ökonomische Ressourcen der Angehörigen der EU-Anwerbeländer aber durchgängig besser als die Ressourcen der Einwanderer aus der Türkei oder dem früheren Jugoslawien. ${ }^{12}$ Dies zeigt sich ers-

108 Die Erhebungswelle 1986 erfaßte 10645 Personen, darunter 1190 Angehörige der EU-Anwerbeländer, 865 Angehörige der Türkei und 459 Angehörige Jugoslawiens (insgesamt: 2514 Personen, 23,5\% der Stichprobe). Zum Erhebungszeitpunkt waren fast alle Befragten bereits länger als 10 Jahre in Deutschland (spanische Staatsangehörige: 96\%; italienische Staatsangehörige: $80 \%$; griechische Staatsangehörige: $90 \%$; Staatsangehörige aus dem früheren Jugoslawien: 90\%; Staatsangehörige der Türkei: $68 \%$ ). Die Befragten der Erhebungswelle 1986 waren $\mathrm{zu}$ einem großen Teil in den späten 1960er Jahren und frühen 1970er Jahren eingereist.

109 Der Anteil derer, die keinen Schulabschluß hatten, lag bei den spanischen Staatsangehörigen bei 33\%, bei den italienischen Staatsangehörigen bei 37\%, bei den griechischen Staatsangehörigen bei $31 \%$, bei den türkischen Staatsangehörigen bei $37 \%$ und bei den jugoslawischen Staatsangehörigen bei 29\%. Bei den deutschen Befragten lag der Anteil bei 3\%. Vgl. auch Anhang, Tabelle 2. Über keinen Berufsabschluß verfügten $56 \%$ der spanischen Staatsangehörigen, 66\% der italienischen Staatsangehörigen, 69\% der griechischen Staatsangehörigen, 69\% der türkischen Staatsangehörigen und $51 \%$ der jugoslawischen Staatsangehörigen. Bei den Deutschen lag der Anteil bei $26 \%$. Vgl. auch Anhang, Tabelle 3.

${ }^{110}$ Im Jahr 1986 waren 43,3\% der deutschen Befragten nicht erwerbstätig, in der Gruppe der Angehörigen der EU-Anwerbeländer waren es nur 28,1\%, in der Gruppe der Angehörigen Jugoslawiens 26,8\%. Der Anteil der nicht-erwerbstätigen türkischen Befragten lag relativ hoch bei $42 \%$.

111 Nach den Daten der Erhebungswelle 1986 unterscheiden sich die Brutto-Einkommen der Einwanderer aus den Anwerbeländern in den ersten drei Quintilen kaum voneinander. In diesen Quintilen bestehen auch kaum Unterschiede zu den Erwerbseinkommen der Deutschen. In der vierten und fünften Quintile sind die Einkommen der Deutschen deutlich höher. Im Verhältnis der Einwanderer aus den Anwerbeländern untereinander zeigen sich in der vierten Quintile leichte Vorteile zugunsten der Einwanderer aus dem früheren Jugoslawien, in der fünften Quintile deutlichere Vorteile zugunsten der Angehörigen der EU-Anwerbeländer.

112 Die Erhebungswelle 1996 erfaßte 13511 Personen, darunter 768 Angehörige der EU-Anwerbeländer, 784 Angehörige der Türkei und 432 Angehörige des früheren Jugoslawien (insgesamt 1984 Personen). Die Erhebungswelle 2006 erfaßte 22332 Perso- 
tens an der Qualifikation: Zwischen 1986 und 2006 nimmt der Anteil derer, die die Fachhochschul- bzw. Hochschulreife erlangen, bei den Angehörigen der EU-Anwerbeländer stärker zu als bei den Angehörigen der anderen Anwerbeländer (Anhang, Tabelle 2). ${ }^{113}$ Der Anteil derer, die über keinen Berufsabschluss verfügen, sinkt bei den EU-Angehörigen stärker als bei den Einwanderern aus der Türkei oder dem früheren Jugoslawien (Anhang, Tabelle 3). Zweitens: Die Erwerbseinkommen der Angehörigen der EU-Anwerbeländer sind in allen Einkommensgruppen höher als die der Angehörigen des früheren Jugoslawien oder der Türkei (Anhang, Tabellen 5, 6). Im Jahr 2006 entsprechen die Erwerbseinkommen der EU-Angehörigen bis in die dritte Quintile etwa den Einkommen der Deutschen. Die Angehörigen der anderen Anwerbeländer bleiben schon in der zweiten Quintile hinter den Deutschen zurück. ${ }^{114}$ Aus den Daten folgt nicht zwingend, daß der Rechtsstatus die soziale Lage bestimmt. Die Daten legen einen Zusammenhang gleichwohl nahe. Diejenigen, die in höherem Maß in die Funktionssysteme des Rechts - auch des Sozialrechts - inkludiert sind, erreichen bessere soziale Positionen. Ein erhöhtes $\mathrm{Ma}$ an Sicherheit, auch sozialer Sicherheit, kann auf der Ebene des individuellen Verhaltens sichtlich in mehr soziale Gleichheit umgemünzt werden.

nen, darunter 407 Angehörige der EU-Anwerbeländer, 479 Angehörige der Türkei und 235 Angehörige eines Nachfolgestaates des früheren Jugoslawien (insgesamt: 1121 Personen). Im Zeitpunkt der Befragung 2006 hielt sich der überwiegende Teil der Befragten bereits mehr als 30 Jahre in Deutschland auf (spanische Staatsangehörige: $88 \%$; italienische Staatsangehörige: 69\%; griechische Staatsangehörige: $78 \%$; Staatsangehörige eines Nachfolgestaates des früheren Jugoslawien: $57 \%$; Staatsangehörige der Türkei: $43 \%)$.

113 Die SOEP-Daten bestätigen damit die Ergebnisse von U. Mehrländer/C. Ascheberg/J. Ueltzhöffer Repräsentativuntersuchung '95 (Fn. 106) 29, 61.

114 Bemerkenswert ist, daß der Berufsstatus der Einwanderer aus den Anwerbeländern dem Rechtsstatus der Betroffenen nicht so stark entspricht (vgl. dazu Anhang, Tabelle 7). Der Anteil derer, die sich bei der Befragung den „Arbeitern“ zuordnen, nimmt zwar bei den Angehörigen der EU-Anwerbeländern viel stärker ab als bei den Angehörigen der Türkei; der Anteil der Angestellten nimmt stärker zu. Bei den Angehörigen aus einem der Nachfolgestaaten des früheren Jugoslawien läuft die Entwicklung aber sehr ähnlich wie bei den Angehörigen der EU-Anwerbeländern. Bei den Angehörigen des früheren Jugoslawien dürfte der günstig verlaufende Statuswechsel auf ihren frühen Statusvorsprung zurückzuführen sein. Einwanderer aus dem früheren Jugoslawien sind seit den 1960er Jahren bei den ungelernten und angelernten Arbeitern schwächer vertreten als die Vergleichsgruppen. Sie hielten stets einen großen Anteil bei den Facharbeitern, Vorarbeitern und bei den unteren Angestellten. Diese besondere Ausgangslage dürfte die spätere günstige Entwicklung maßgeblich bestimmt haben. Näher $U$. Mehrländer/C. Ascheberg/J. Ueltzhöffer Repräsentativuntersuchung '95 (Fn. 106) $71 \mathrm{ff}$. 
Meine achte These lautet: Es gibt einen plausiblen Zusammenhang zwischen der strikten Inklusionspolitik der Gemeinschaft und der Lage der Arbeitskräfte aus den EU-Anwerbeländern. Ihre Lage unterscheidet sich noch immer von der der Deutschen. Sie haben sich in ihren Ressourcen den Deutschen indes mehr angenähert als die Angehörigen der anderen Anwerbeländer. Anders gewendet: Die Aufgabe der Gemeinschaft bezieht sich auf rechtliche Gleichheit. Rechtliche Gleichheit bewirkt hier jedoch zugleich mehr soziale Gleichheit.

\section{Globale soziale Ungleichheit: Verantwortung für die Welt}

\section{Neue Semantiken}

Nach verbreiteter Auffassung bilden Staaten Solidargemeinschaften. ${ }^{115}$ Solidarität bezieht sich auf die eigenen Angehörigen und endet an den Staatsgrenzen. Das Gemeinschaftsrecht hat die so verstandene Solidarität für Freizügigkeitsberechtigte ein Stück weit aufgebrochen. Noch radikaler wird herkömmliche Solidarität auf der globalen Ebene in Frage gestellt. Der rechtliche Diskurs läuft wiederum parallel zu einem philosophischen und einem soziologischen. Der philosophische Diskurs versucht, internationale Solidarität moralisch zu begründen. ${ }^{116}$ Der soziologische beschäftigt sich mit der Überwindung des „methodischen Nationalismus“ und der Entstehung einer globalen Sozialpolitik. ${ }^{117}$ Ich will die Frage aufgreifen, ob Industriestaaten recht-

115 Aus rechtlicher Perspektive z.B. Th. Kingreen Das Sozialstaatsprinzip im europäischen Verfassungsverbund. Gemeinschaftsrechtliche Einflüsse auf das deutsche Recht der gesetzlichen Krankenversicherung, 2003, 71 ff., $244 \mathrm{ff}$.; E. Wiederin Sozialstaatlichkeit im Spannungsfeld von Eigenverantwortung und Fürsorge, VVDStRL 64 (2005) 53; J. Isensee (Fn. 18) § 15; H. Maurer Staatsrecht I. Grundlagen, Verfassungsorgane, Staatsfunktionen, 5. Aufl. 2007, § 1 Rn. 11 ff. Aus soziologischer Perspektive etwa M. Heidenreich Die Europäisierung sozialer Ungleichheiten zwischen nationaler Solidarität, europäischer Koordinierung und globalem Wettbewerb, in: M. Heidenreich (Hrsg.) Die Europäisierung sozialer Ungleichheit. Zur transnationalen Klassen- und Sozialstrukturanalyse, 2006, 17.

116 Dazu insb. Th. Nagel (Fn. 10); Th. Pogge (Fn. 10); die Beiträge in: A. Follesdal/ Th. Pogge (Hrsg.) Real World Justice. Grounds, Principles, Human Rights, and Social Institutions, 2005, und in: Th. Pogge (Hrsg.) Freedom from Poverty as a Human Right. Who owes what to the very poor?, 2007. Vgl. auch A. Sen Elements of a Theory of Human Rights, Philosophy and Public Affairs 32/4 (2004) 315; P. Vizard Poverty and Human Rights. Sen's ,Capability Perspective‘ Explored, 2006.

117 Für die Ungleichheitsforschung vgl. R. Kreckel Soziologie der sozialen Ungleichheit im globalen Kontext, in: M. Bayer/G. Mordt/S. Terpe/M. Winter (Hrsg.) Transnationale Ungleichheitsforschung. Eine neue Herausforderung für die Soziologie, 2008, 
lich verpflichtet sind, zur Bekämpfung der Armut in anderen Erdteilen beizutragen. ${ }^{118}$ Meine Frage knüpft zwar an die Vorstöße der Blockfreien aus der Mitte der 1970er Jahre an. ${ }^{119}$ Die politischen

23. Für die Sozialpolitik vgl. B. Deacon/M. Hulse/P. Stubbs Global Social Policy. International Organizations and the Future of Welfare, 1997; B. Deacon Global Social Policy \& Governance, 2007; A. Hall/J. Midgley Social Policy for Development, 2004 (reprint 2006); L. Leisering/P. Buhr/U. Traiser-Diop Soziale Grundsicherung in der Weltgesellschaft. Monetäre Mindestsicherungssysteme in den Ländern des Südens und des Nordens, 2006; L. Leisering Gibt es einen Weltwohlfahrtsstaat?, in: M. Albert/R. Stichweh (Hrsg.) Weltstaat und Weltstaatlichkeit. Beobachtungen globaler politischer Strukturbildung, 2007, 185; L. Leisering Soziale Globalisierung? Die Entstehung globaler Sozialpolitik, Aus Politik und Zeitgeschichte 21 (2008) 21; W. S. Kim Die Entstehung „neuer Wohlfahrtsstaaten“ und globale Policy-Diffusion - das Beispiel Südkorea, Zeitschrift für Soziologie 37/3 (2008) 186.

${ }_{118}$ Der rechtliche Diskurs ist breiter. Er betrifft (allgemein) das Verhältnis von Armut, Entwicklungspolitik und Menschenrechten. Aus der Perspektive der Entwicklungspolitik vgl. insb. A. Sengupta On the Theory and Practice of the Right to Development, HRQ 24 (2002) 837; St. P. Marks The Human Rights Framework for Development: Seven Approaches, 2003, abrufbar unter: www.hsph.harvard.edu/ fxbcenter/FXBC_WP18--Marks.pdf; A. Sengupta Poverty Eradication and Human Rights, 2004, abrufbar unter: http://www.hsph.harvard.edu/fxbcenter/No.20_Sengputa.pdf; M. Darrow/A. Tomas Power, Capture, and Conflict: A Call for Human Rights Accountability in Development Cooperation, HRQ 27 (2005) 471. Aus der Perspektive der Menschenrechte vgl. insb. S. I. Skogly/M. Gibney Transnational Human Rights Obligations, HRQ 24 (2002) 781; M. Nowak A Human Rights Approach to Poverty, in: M. Scheinin/M. Suksi (Hrsg.) Human Rights in Development Yearbook 2002, 2005, 15; Ph. Alston Ships Passing in the Night: The Current State of the Human Rights and Development Debate Seen Through the Lens of the Millenium Development Goals, HRQ 27 (2005) 755; M. Robinson What Rights Can Add to Good Development Practice, in: Ph. Alston/M. Robinson (Hrsg.) Human Rights and Development. Towards Mutual Reinforcement, 2005, 25; kritisch H. Dean Social Policy and Human Rights: Re-thinking the Engagement, Social Policy \& Society 7 (2008) 1; J. Donnelly Human Rights and Social Provision, Journal of Human Rights 7 (2008) 123.

119 Im Mai und Dezember 1974 setzten die Blockfreien in der Generalversammlung gegen den Widerstand der Industriestaaten mehrere Resolutionen durch, mit denen eine „Neue Weltwirtschaftsordnung“ begründet werden sollte. Vgl. Declaration on the Establishment of a New International Economic Order, General Assembly Resolution 3201 (S-VI), 1 May 1974; Programme of Action on the Establishment of a New International Economic Order, General Assembly Resolution 3202 (S-VI), 1 May 1974; Charter of Economic Rights and Duties of States, General Assembly Resolution 3281 (XXIX), 12 December 1974; Development and international economic co-operation, General Assembly Resolution 3362 (S-VII), 16 September 1975. Die „Neue Weltwirtschaftsordnung" richtete sich gegen Kolonialismus und alle Formen von Neo-Kolonialismus; sie zielte v. a. auf eine Beseitigung der bestehenden globalen Ungleichheiten, die insbesondere die Entwicklungsländer belasten würden. Die Blockfreien forderten: internationale Zusammenarbeit mit dem Ziel der Verringerung der Ungleichheit; volle Kontrolle über die natürlichen Ressourcen auf dem Staatsgebiet; ein Recht auf Entschä- 


\section{Rahmenbedingungen der Diskussion haben sich aber völlig verän- dert. ${ }^{120}$}

digung für die Ausbeutung durch andere Staaten; Verbesserung der terms of trade zugunsten der Entwicklungsländer; eine Entwicklungshilfe, die nicht an wirtschaftliche oder politische Bedingungen geknüpft ist; einen Technologietransfer von den entwickelten Staaten zu den Entwicklungsländern.

120 Die Umsetzung der „Neuen Weltwirtschaftsordnung“ scheiterte am Widerstand der Industrienationen. Im Jahr 1989 hielt der Generalsekretär der Vereinten Nationen in seinem (letzten) Implementationsbericht fest, daß die Grundsätze und Leitlinien des Jahres 1974 weitgehend unerfüllt geblieben seien (U.N. Doc A/44/266, 19 May 1989). Der Fall des Eisernen Vorhangs und das Ende des Kommunismus in Osteuropa brachten Bewegung in die festgefahrenen Diskussionen. Das Thema „Entwicklung“ war von den überkommenen ideologischen Frontstellungen befreit. Am 1. Mai 1990 nahm die Generalversammlung der Vereinten Nationen eine weitere Deklaration über die internationale Zusammenarbeit an, die richtungsweisend für das kommende Jahrzehnt sein sollte (Declaration on International Economic Cooperation, in particular the Revitalization of Economic Growth and Development of the Developing Countries, Res. S-18/3). Freilich, die für die 1990er Jahre ins Auge gefaßte Entwicklungspolitik entsprach nicht vollständig den Vorstellungen der Blockfreien aus dem Jahr 1974: Die Deklaration aus dem Jahr 1990 enthält keine Stellungnahme zum Kolonialismus bzw. NeoKolonialismus. Sie spricht nicht von einem bedingungslosen Recht auf Entwicklungshilfe. Die Verbindlichkeiten der Deklaration (commitments) greifen gleichwohl viele Inhalte der „Neuen Weltwirtschaftsordnung“ auf: So werden die Industriestaaten etwa aufgefordert, für nachhaltiges Wachstum und eine Verringerung der globalen sozialen Ungleichheit zu sorgen. Die Industrienationen werden weiter aufgefordert, die Entwicklungshilfe entsprechend ihrer Zusage auf 0,7\% des BNP anzuheben. Es wird auf die Wichtigkeit von Wissens- und Technologietransfers hingewiesen. Und es wird gefordert, für ein Welthandelssystem zu sorgen, das auf Protektionismus weitgehend verzichtet. All das sind der Sache nach Forderungen aus dem Jahr 1974. Ende des Jahres 1990 wurden auf der Basis der Deklaration sechs konkrete Entwicklungsziele formuliert (International Development Strategy for the Fourth United Nations Development Decade, General Assembly Resolution, A/RES/45/199, 21 December 1990): surge in the pace of economic growth; reduction in extreme poverty; improvements of the international systems of money, finance and trade; sound macro-economic management; strengthening of international development co-operation. Ein weiteres Jahrzehnt später konnte der Generalsekretär der Vereinten Nationen in seinem Implementationsbericht wohl einige Erfolge verzeichnen, insb. für die lateinamerikanischen Staaten und Staaten in Südost-Asien (A/55/209, 31 July 2000). Die Zahl derer, die von weniger als einem US Dollar am Tag leben müßten, liege aber immer noch bei 1,2 Mrd. Die Entwicklungshilfe sei in den 1990er Jahren stetig zurückgegangen. Der Bericht empfahl daher, für das kommende Jahrzehnt eine neue Strategie zu entwerfen. Die Deklaration zur Jahrtausendwende enthielt sodann eine Reihe neuerlicher Versprechungen, insb. auch mit Blick auf Armut: „We will spare no effort to free our fellow men, women and children from the abject and dehumanizing conditions of extreme poverty, to which more than a billion of them are currently subjected. We are committed to making the right to development a reality for everyone and to freeing the entire human race from want" (United Nations Millennium Declaration, A/RES/55/2, 18 September 2000). In den Millennium Development 
Auf der Ebene der Vereinten Nationen wurde die Verantwortung aller Staaten für die Armutsbekämpfung in der Welt erstmals in einem Expertenbericht aus dem Jahr 2004 angesprochen. ${ }^{121}$ Die Sicherheit von Menschen (human security) sei heute - so hieß es dort - nicht mehr so sehr von aggressiven Staaten bedroht. Heute sei Armut eine der zentralen Bedrohungen für die Menschenrechte. ${ }^{122}$ Und wenngleich die Staaten die Hauptverantwortung für die Sicherheit in ihren Territorien tragen, sei die Staatengemeinschaft doch subsidiär zuständig. Diese „collective responsibility to protect“" verpflichte dazu, schwachen Staaten bei der Herstellung der Fähigkeit zu helfen, mit sozialen Bedrohungen umzugehen. ${ }^{123}$ Der Weltgipfel 2005 griff das Konzept auf. ${ }^{124}$ Im

Goals scheint die Bekämpfung von weltweiter Armut als erstes und wichtigstes Ziel auf (Road map towards the implementation of the United Nations Millennium Declaration, Report of the Secretary-General, A/56/326, 6 September 2001): „Eradicate extreme poverty and hunger".

${ }^{121}$ Report of the High-level Panel on Threats, Challenges and Change, U.N. Doc. A/59/565, 2 December 2004. Die Expertengruppe unter dem Vorsitz des thailändischen Regierungschefs wurde vom Generalsekretär der Vereinten Nationen (Kofi Annan) ins Leben gerufen. Die Experten sollten sich einer Frage widmen, die nach Auffassung des Generalsekretärs bei den Staaten der Welt umstritten war. Kofi Annan sprach von „deep divisions among the Member States on the nature of the threats that we [face] and the appropriateness of the use of force to address those threats" (ebd. 1). Der Generalsekretär erhoffte sich, daß der Bericht die Diskussion strukturieren und einige konsensfähige Antworten erbringen würde, die sodann zu einer Stärkung der Vereinten Nationen führen könnten. Die Expertengruppe der Vereinten Nationen konnte ihrerseits auf einen Expertenbericht zurückgreifen: Report of the International Commission on Intervention and State Sovereignty (ICISS), The Responsibility to Protect, 2001. Der Bericht aus dem Jahr 2001 stammte von einer Gruppe, die von der kanadischen Regierung beauftragt worden war, Möglichkeiten und Grenzen einer humanitären Intervention zu untersuchen. Daß die Überlegungen letztlich auch Maßnahmen nach Kapitel VII der Satzung der Vereinten Nationen legitimieren könnten, hat das Nachdenken über „Bedrohungen“ politisch heikel gemacht.

122 Unter der Überschrift „Towards a new security consensus“ hielt die Expertengruppe (Fn. 121) 11 fest: „The United Nations was created in 1945 above all else, to save succeeding generations from the scourge of war' - to ensure that the horrors of the World Wars were never repeated. Sixty years later, we know all too well that the biggest security threats we face now, and in the decades ahead, go far beyond States waging aggressive war. They extend to poverty, infectious disease and environmental degradation; war and violence within States; the spread and possible use of nuclear, radiological, chemical and biological weapons; terrorism; and transnational organized crime. The threats are from non-State actors as well as States, and to human security as well as State security.“

${ }^{123}$ Ebd. 22.

124 World Summit Outcome, A/RES/60/1. In der Resolution der Generalversammlung zum Weltgipfel 2005 scheint die internationale Schutzverantwortung (responsibility to protect) auf bestimmte Bedrohungen verengt zu sein, nämlich auf Bedrohungen durch 
Februar 2008 bestellte der Generalsekretär der Vereinten Nationen einen eigenen Sonderbeauftragten. ${ }^{125}$ Die Semantik der internationalen Schutzverantwortung ist in mehrfacher Hinsicht neu: Neu ist, dass „freedom from want“ gleich oder höher gewichtet wird als „freedom from fear". ${ }^{26}$ Neu ist, dass der schwache Staat (nicht nur der starke Staat) als Urheber von Menschenrechtsverletzungen gilt. ${ }^{127}$ Und neu ist, dass in den Souveränitätsbegriff Verantwortlichkeiten hineingelesen werden, die - bei Versagen des Staates - auf andere Staaten übergehen. ${ }^{128}$

Völkermord, Kriegsverbrechen, ethnische Säuberungen und Verbrechen gegen die Menschlichkeit (ebd. para. 138). Die Verengung dürfte darauf zurückzuführen sein, daß nur mit Blick auf diese Bedrohungen die Bezugnahme auf Maßnahmen nach Kapitel VII der Satzung der Vereinten Nationen ausreichend konsensfähig war. Die Verantwortung der Staaten wie der Vereinten Nationen für die Armutsbekämpfung wird an anderen Stellen unterstrichen (ebd. para. 17, 19, 24, 27, 35, 38). Im Begriff der „human security“ aber bleiben beide Schutzgüter (freedom from want, freedom from fear) zusammengeführt (ebd. para. 143): „We stress the right of people to live in freedom and dignity, free from poverty and despair. We recognize that all individuals, in particular vulnerable people, are entitled to freedom from fear and freedom from want, with an equal opportunity to enjoy all their rights and fully develop their human potential“. Deshalb solle das Konzept von „human security“ in der Generalversammlung weiter behandelt werden.

${ }^{125}$ Edward Luck wurde am 21. 2. 2008 von Ban Ki-moon zum Sonderbeauftragten für die internationale Schutzverantwortung bestellt. Vgl. das Schreiben des Generalsekretärs, S/2007/721, 7 December 2007, und den Bericht des Generalsekretärs, A/HRC/7/37, 18 March 2008.

${ }^{126}$ Soweit zu sehen, war es Kofi Annan, der die Ausdrücke „freedom from want“ und „freedom from fear" wieder aufgegriffen hat. In einem Bericht, der dem MillenniumsGipfel vorangegangen war, spielen diese beiden Schutzgüter eine zentrale Rolle (Report of the Secretary-General, We the peoples: the role of the United Nations in the twenty-first century, A/54/2000, 27 March 2000). Kofi Annan konnte damit an eine Rede des amerikanischen Präsidenten Theodore Roosevelt vom 6. 1. 1941 und an die Atlantik-Charta vom 14. 8. 1941 anknüpfen, in der die Alliierten ihre Kriegsziele formulierten. Bei beiden Gelegenheiten wurde die Bedeutung von sozialer Sicherheit für den Frieden hervorgehoben. In dieser Betonung - das gilt für 1941 ebenso wie für 2000 und danach - liegt eine Absage an die Auffassung, daß soziale Rechte inferior und kein legitimer Gegenstand internationaler Verpflichtungen seien.

${ }^{127}$ Dem „schwachen Staat“ gilt heute die besondere Aufmerksamkeit der sozialwissenschaftlichen Forschung, gerade auch unter dem Blickwinkel der Einhaltung der staatlichen Verpflichtungen auf der Grundlage der Menschenrechte. Vgl. jüngst die Beiträge in: Th. Risse/U. Lehmkuhl (Hrsg.) Regieren ohne Staat? Governance in Räumen begrenzter Staatlichkeit, 2007.

${ }^{128}$ In dieser subsidiären Zuständigkeit der Staatengemeinschaft (verkörpert durch die Vereinten Nationen) und anderen Staaten liegt ein Spannungsverhältnis zum völkerrechtlichen Interventionsverbot und zum Gewaltverbot. Auf der Ebene der Vereinten Nationen gibt es offenbar Bestrebungen, die internationale Schutzverantwortung auf besonders schwere Völkerrechtsverletzungen einzuschränken (vgl. oben in Fn. 124). Da 


\section{Neue Rechtsnormen?}

Was die rechtliche Fundierung der internationalen Schutzverantwortung betrifft, verweisen die Dokumente zumeist auf eine im Entstehen begriffene Norm des Völkerrechts. ${ }^{129}$

In der Tat bieten die Regeln über die Staatenverantwortlichkeit (state responsibility) keine Grundlage: Staatenverantwortlichkeit knüpft an eine Völkerrechtsverletzung an,130 Schutzverantwortung soll schon im Vorfeld greifen. Staatenverantwortlichkeit setzt eine zurechenbare Rechtsverletzung und einen Staat voraus, der in der Lage ist, Völkerrecht zu beachten. ${ }^{131}$ Schutzverantwortung wird gerade auch dann aktiviert, wenn dies nicht der Fall ist. Staatenverantwortlichkeit nimmt die Staaten der Welt nur in die Pflicht, wenn eine zwingende Norm des Völkerrechts verletzt wurde, und zwar auf schwerwiegende Weise. ${ }^{132}$ Schutzverantwortung greift demgegenüber immer schon dann, wenn (fremde) Staatsgewalt versagt. Schließlich: Bei Verletzung von erga omnes-Pflichten sollen die Staaten den verletzenden Staat zum Einlen-

es im Kontext von „freedom from want“ primär um präventive Maßnahmen geht und nicht um Maßnahmen nach Kapitel VII der Satzung der Vereinten Nationen, will ich das Spannungsverhältnis nicht weiter verfolgen. Im übrigen ist die Literatur zur internationalen Schutzverantwortung noch spärlich. Vgl. H. Corell From Territorial Sovereignty to Human Security, 1999, abrufbar unter: http://www.un.org/law/counsel/ottawa.htm; H. Corell To intervene or not: The dilemma that will not go away, 2001, abrufbar unter: http://untreaty.un.org/OLA/legal_counsel5.aspx; R. J. Hamilton Recent Development: The Responsibility to Protect: From Document to Doctrine - But what of Implementation? Harv. Hum. Rts. J. 19 (2006) 289; A.-M. Slaughter/W. BurkeWhite The Future of International Law is Domestic (or, The European Way of Law), Harv. Int'l L.J. 47 (2006) 327; Ch. C. Joyner „The Responsibility to Protect“: Humanitarian Concern and the Lawfulness of Armed Intervention, Va. J. Int'1 L. 47 (2007) 693; C. Stahn Responsibility to Protect: Political Rhetoric or Emerging Legal Norm? AJIL 101/1 (2007) 99; Th. Rensmann Die Humanisierung des Völkerrechts durch das ius in bello - Von der Martens'schen Klausel zur „Responsibility to Protect“, ZaöRV 68 (2008) 111.

${ }^{129}$ Z.B. Expertenbericht (Fn. 121) 57: „emerging norm of a collective international responsibility to protect“".

130 Art. 2 Draft Articles on the Responsibility of States for Internationally Wrongful Acts, ILC, General Assembly Resolution A/RES/56/83, 12 December 2001, Annex (im folgenden: Draft Articles).

131 Art. 2 (,attributable to the State“), Art. 23 („force majeure“), Art. 24 („distress“), Art. 25 („necessity“) Draft Articles. Staatenverantwortlichkeit greift auch dann nicht, wenn ein Staat - mit Blick auf soziale Rechte - geltend machen kann, er verfüge nicht über die nötigen Ressourcen, um die Verpflichtung einzuhalten. Das Argument wird ausdrücklich zugelassen durch die Vorbehalte in Art. 2 Abs. 1 und Art. 11 Abs. 1 Sozialpakt der Vereinten Nationen.

132 Art. 40, 41 Draft Articles. 
ken bewegen. ${ }^{133}$ Die internationale Schutzverantwortung verlangt, dass Staaten andere Staaten zur Einhaltung von Völkerrecht befähigen.

Das bedeutet jedoch nicht, dass sich die internationale Verantwortung zur Bekämpfung von globaler Armut nicht an existierendes Völkerrecht anbinden lässt.

Erstens: Der Souveränitätsbegriff ist schon lange kein „westfälischer“ mehr. ${ }^{134}$ Die Satzung der Vereinten Nationen macht unmissverständlich klar, dass die Staaten Verantwortung übernehmen, insbesondere für die Verwirklichung der Ziele der Vereinten Nationen, und damit für die Achtung der Menschenrechte. ${ }^{135}$ Zweitens: Die Annahme einer Einstandsverantwortung der Vereinten Nationen scheint mir ebenfalls nur eine Zuspitzung der Rechtslage: Der Sicherheitsrat ist wiederholt tätig geworden, wenn die materiellen Bedürfnisse von Menschen nicht sichergestellt waren. ${ }^{136}$ Drittens: Armutsbekämpfung hat eine menschenrechtliche Grundlage. Das Recht auf einen angemessenen Lebensstandard nach Art. 11 des Sozialpakts läuft bei extremer Armut leer. Menschen haben dann regelmäßig keinen Zugang zu Nahrung, Kleidung oder Wohnen. ${ }^{137}$ Art. 11 des Sozialpaktes wiederum nimmt die

133 Art. 41, 48 Draft Articles. Der IGH erkennt erga omnes-Pflichten nur sehr zurückhaltend an. Vgl. zuletzt ICJ, advisory opinion, 9 July 2004, Legal Consequences of the Construction of a Wall in the Occupied Palestinian Territory, para. 155-160.

${ }^{134}$ So aber z.B. H. Corell Territorial Sovereignty (Fn. 128); ICISS (Fn. 121) 12; vgl auch noch den Expertenbericht (Fn. 121) 21.

${ }_{135}$ Art. 4 Abs. 1 der Satzung der Vereinten Nationen bestimmt: „Membership in the United Nations is open to all ... peace-loving states which accept the obligations contained in the present Charter and, in the judgment of the Organization, are able and willing to carry out these obligations". Nach Art. 2 Nr. 5 der Satzung sind alle Mitgliedstaaten verpflichtet, die Organisation bei der Verwirklichung ihrer Ziele zu unterstützen. Nach Art. 1 der Satzung zählt die Förderung der Menschenrechte zu den Zielen der Vereinten Nationen.

${ }^{136}$ Im Jahr 1992 ergingen die ersten Resolutionen des Sicherheitsrates zur Hilfe von Menschen in materieller Not. Die Resolutionen betrafen die Menschen in Somalia (S/RES/767, 27 July 1992; S/RES/794, 3 December 1992). Im Jahr 1996 betraf die Hilfe Menschen in der Great Lakes Region (S/RES/1078, 9 November 1996; S/RES/1080, 15 November 1996), im Jahr 2004 Menschen in Haiti (S/RES/1529, 29 February 2004; S/RES/1542, 30 April 2004) und im Sudan (S/RES/1556, 30 July 2004), im Jahr 2007 Menschen in Haiti und in der Demokratischen Republik Kongo (S/RES/1743, 15 February 2007; S/RES/1756, 15 May 2007; S/RES/1780, 15 October 2007). Alle angeführten Resolutionen gründeten sich auf Kapitel VII der Satzung der Vereinten Nationen.

137 Art. 11 Abs. 1 S. 1 Sozialpakt bestimmt: „The States Parties to the present Covenant recognize the right of everyone to an adequate standard of living for himself and his family, including adequate food, clothing and housing, and to the continuous improvement of living conditions“. Der Sozialpakt der Vereinten Nationen gehört zu den am meisten ratifizierten völkerrechtlichen Verträgen. Der Vertrag hat derzeit 159 Mitglied- 
Staaten nicht isoliert in die Pflicht, sondern verweist sie auf internationale Zusammenarbeit: Die Vertragsstaaten haben - so heißt es dort geeignete Schritte zur Verwirklichung des Rechts auf angemessenen Le-

staaten. Darunter befinden sich alle Industriestaaten der Welt, ausgenommen die USA. Die Menschenrechtsorgane der Vereinten Nationen machen im Fall von extremer Armut die Menschenrechtsverletzung nicht nur an einem einzigen Menschenrecht fest; sie bevorzugen einen holistischen Zugang (vgl. etwa Office of the United Nations High Commissioner for Human Rights Principles and Guidelines for a Human Rights Approach to Poverty Reduction Strategies, 2006, Foreword: Poverty „describes a complex of interrelated and mutually reinforcing deprivations, which impact on people's ability to claim and access their civil, cultural, economic, political and social rights. In a fundamental way, therefore, the denial of human rights forms part of the very definition of what it is to be poor"). Der breite, alle Menschenrechte umfassende Zugang ist auf den ersten Blick durchaus plausibel: In einem Land, in dem $50 \%$ der Bevölkerung oder mehr unterhalb der Armutsgrenzen von einem oder zwei US Dollar am Tag leben, sind mit groBer Wahrscheinlichkeit viele Menschenrechte nicht sichergestellt. Armut bedeutet oft, da $ß$ die Rechte auf Bildung, auf Arbeit, auf Gesundheit oder auf Beteiligung an der politischen Willensbildung nicht wahrgenommen werden können oder das Recht auf Leben nicht sichergestellt ist. Extreme Armut geht vielfach einher mit Analphabetismus, Arbeitslosigkeit, chronischen Krankheiten, frühem Tod. Es trifft weiter zu, daß die Gerichte verschiedener Länder Armut bzw. Folgen von Armut z. B. unter dem Blickwinkel des Rechts auf Leben aufgreifen (vgl. die Nachweise bei P. Vizard [Fn. 116] 176). Und richtig ist auch, daß Armut bekämpft oder vermieden werden kann, wenn - um zwei Beispiele zu nennen - das Recht auf Arbeit oder das Recht auf soziale Sicherheit gewährleistet ist. Dennoch: In seinem Kern wird Armut oft beschrieben als das Fehlen der Möglichkeit, bestimmte Grundbedürfnisse zu decken (vgl. A. Sengupta Poverty Eradication [Fn. 118] 13; Copenhagen Declaration on Social Development, in: U.N. Doc. A/CONF.166/9, 19 April 1995, Commitment 2b). Das Entwicklungsprogramm der Vereinten Nationen (UNDP) geht in eine ähnliche Richtung. UNDP setzt „freedom from want" gleich mit einem Zustand, in dem ein menschenwürdiger Lebensstandard nicht verfügbar ist, insb. mit Blick auf Nahrung, Wohnen, Zugang zu Wasser, Zugang zu sanitären Anlagen (UNDP Human Development Report 2000, Human Rights and Human Development, 2000, 1, 3, 31,33). Dies entspricht der auch im Westen verbreiteten Vorstellung, „Grundsicherung“ umfasse Bedarfsdeckung primär im Bereich der Ernährung, der Kleidung und des Wohnens. Hier setzt Hilfeleistung auch sinnvollerweise zunächst an: Hungrige Kinder werden nicht allein deshalb satt, weil sie in die Schule gehen. Als allgemeines, auf Grundsicherung zugeschnittenes Recht steht das Recht aus Art. 11 des Sozialpaktes daher im Mittelpunkt der Armutsbekämpfung. Armutsbekämpfungsmechanismen, die sich anderen Rechten des Sozialpaktes zuordnen lassen, sind demgegenüber spezieller. Rechte des internationalen Paktes über bürgerliche und zivile Rechte (International Covenant on Civil and Political Rights, General Assembly Resolution 2200 A [XXI]) wiederum können - je nach Sachlage - zusätzlich einschlägig sein (Recht auf Leben, Art. 6 Abs. 1). So verstanden, bildet Art. 11 Sozialpakt zugleich ein wesentliches Element des Rechts auf Entwicklung (right to development). Zum Recht auf Entwicklung vgl. insb. A. Sengupta Theory and Practice (Fn. 118); Ph. Alston Making Space for New Human Rights: The Case of the Right to Development, Harv. Hum. Rts. Y.B. 1 (1988) 3. 
bensstandard $\mathrm{zu}$ setzen und sie haben dabei die entscheidende Bedeutung der internationalen Zusammenarbeit zu beachten. ${ }^{138}$ Dass $\mathrm{Zu}-$ sammenarbeit zur Armutsbekämpfung nicht nur eine Sache der Nehmerländer ist, sondern vor allem auch der Geberländer, kann sich auf einen langjährigen Konsens stützen, der mittlerweile von einer Rechtsüberzeugung getragen ist. Das zeigt insbesondere die Staatenpraxis in den Bereichen Entwicklungspolitik und internationale wirtschaftliche Zusammenarbeit, die seit 2002 ein gemeinsames Dach im Monterrey Consensus gefunden haben. ${ }^{139}$

138 Art. 11 Abs. 1 S. 2 Sozialpakt bestimmt: „The States Parties will take appropriate steps to ensure the realization of this right, recognizing to this effect the essential importance of international co-operation based on free consent". Art. 11 Abs. 1 bekräftigt damit die Rolle der internationalen Zusammenarbeit für ein spezifisches soziales Menschenrecht. Allgemein werden die Staaten bereits in Art. 2 Abs. 1 Sozialpakt auf internationale Zusammenarbeit verwiesen.

139 Alle Resolutionen, die sich auf „Entwicklung“ (development) oder auf wirtschaftliche Zusammenarbeit beziehen, betonen nicht nur die Wichtigkeit der Zusammenarbeit, sie legen Wert auf den verpflichtenden Charakter der Zusammenarbeit. Dies gilt schon für die (umstrittenen) Resolutionen zur „Neuen Weltwirtschaftsordnung“ aus der Mitte der 1970er Jahre (Fn. 119). Es gilt aber - was wichtiger ist - auch für alle einschlägigen Entwicklungen ab 1990. Vgl. Declaration on International Economic Cooperation 1990 (Fn. 120), International Development Strategy for the Fourth United Nations Development Decade (Fn. 120) sowie die Resolutionen zur Implementation der Strategie (A/RES/46/144, 17 December 1991; A/RES/47/152, 18 December 1992; A/RES/48/185, 21 December 1993; A/RES/49/92, 19 December 1994; A/RES/51/ 173, 16 December 1996; A/RES/53/178, 15 December 1998; A/RES/54/206, 22 December 1999; A/RES/55/190, 20 December 2000; A/RES/56/191, 21 December 2001). Im März 2002 einigten sich die Staaten der Welt in Monterrey, Mexico, auf einen Rahmen für eine partnerschaftliche Finanzierung von „Entwicklung“ (Report of the International Conference on Financing for Development, A/CONF.198/11). Deshalb verzichtete die Generalversammlung zu Beginn des Jahrtausends auf einen weiteren Beschluß über eine mittelfristige Entwicklungsstrategie. Es wurde vielmehr beschlossen, die Bemühungen um die Formulierung neuer Entwicklungsziele mit den Follow-upVerhandlungen zum „Monterrey Consensus“ zusammenzulegen: Die Generalversammlung schuf die Grundlagen für einen „High-Level Dialogue“ mit dem Generalauftrag „The Monterrey Consensus: status of implementation and tasks ahead“ (A/RES/57/250, 20 December 2002). An der Notwendigkeit und der Verpflichtung zur internationalen Zusammenarbeit für das Ziel der „Entwicklung“ wurde in keinem dieser Kontexte gezweifelt. Im Gegenteil, es schien naheliegend, die strategischen Fragen mit den Verhandlungen über die Finanzierung zu verschränken. Auch die Resolutionen der Generalversammlung, die sich - seit 1986 - spezifisch mit Entwicklung (development) befassen, beruhen auf der Überzeugung, daß Entwicklung für Entwicklungsländer internationale Zusammenarbeit insbesondere mit willigen Geberländern voraussetzt. In der Deklaration 1986 (Declaration on the Right to Development, A/RES/41/128, 4 December 1986) heißt es in Art. 3 Abs. 3 zunächst allgemein: „States have the duty to co-operate with each other in ensuring development and eliminating 
Vor diesem Hintergrund lautet meine neunte These: Im globalen $\mathrm{Zu}-$ sammenhang wird soziale Ungleichheit zu einer Aufgabe nur dann, aber

obstacles to development“. In Art. 4 Abs. 2 wird dann konkreter festgehalten: „Sustained action is required to promote more rapid development of developing countries. As a complement to the efforts of developing countries, effective international co-operation is essential in providing these countries with appropriate means and facilities to foster their comprehensive development“. Eine ähnliche Sprache findet sich in der Copenhagen Declaration 1995 (Fn. 137; para. 10: „commitment to social development ... and ... international cooperation"), in der Millennium Declaration 2000 (Fn. 120; para. 6: „shared responsibility for ... economic and social development“) oder in der World Summit Declaration 2005 (Fn. 124; para. 20: „global partnership for development“). Die jährlichen Resolutionen zum right to development wurden vielfach ohne formale $\mathrm{Ab}-$ stimmung angenommen (so etwa A/RES/50/184, 22 December 1995; A/RES/51/99, 12 December 1996; A/RES/55/108, 4 December 2000) oder mit großer Mehrheit verabschiedet (so z.B. A/RES/57/223, 18 December 2002; A/RES/58/172, 22 December 2003). In der jüngsten Vergangenheit stießen die Beschlußvorlagen zum right to development allerdings auf einigen Widerstand (vgl. A/RES/61/169, 19 December 2006 [und dazu A/C.3/61/SR.53] sowie A/RES/62/161, 18 December 2007 [und dazu A/C.3/62/SR.54]). Der Widerstand hat einen bestimmten Grund: Seit 1998 arbeitet eine Arbeitsgruppe unter der Ägide des Human Rights Council an der Formulierung von Standards für die Bewertung der internationalen Zusammenarbeit in der Entwicklungspolitik, z.B. im Rahmen der Millennium Development Goals. Diese Arbeitsgruppe nun erwägt - mit Unterstützung des Human Rights Council (Resolution 4/4, 30 March 2007) - die Standards in eine verbindliche Rechtsform zu gießen (vgl. den letzten Bericht der Arbeitsgruppe, A/HRC/4/47, 14 March 2007). Die EU und andere europäische Staaten stehen dem Vorhaben grundsätzlich aufgeschlossen gegenüber. Die Vereinigten Staaten dagegen weisen bei jeder Gelegenheit darauf hin, daß sie einen verbindlichen Text nicht akzeptieren könnten. Resolutionen der Generalversammlung, die das Vorhaben der Arbeitsgruppe unterstützen, werden deshalb nur mehrheitlich verabschiedet, die jüngste (A/RES/62/161) mit 136 Ja-Stimmen gegen 53 Nein-Stimmen. Am überwältigenden Konsens für die Notwendigkeit einer internationalen Zusammenarbeit in der Entwicklungspolitik ändert dies m. E. nichts. Die Deklaration zum right to development wurde 1986 mit $146 \mathrm{Ja}-\mathrm{Stimmen}$ gegen eine Nein-Stimme (Vereinigte Staaten) bei 8 Enthaltungen angenommen. Die Declaration on International Economic Cooperation 1990, die Millennium Declaration 2000 oder die World Summit Declaration 2005 wurden ohne formale Abstimmung beschlossen. Rechtlich konstituieren alle erwähnten Akte aus den vergangenen 20 Jahren eine Staatenpraxis, die inhaltlich konsistent und gleichbleibend Kooperationspflichten bekräftigt, insb. zwischen Industriestaaten und Entwicklungsländern. Spätere Entscheidungen nehmen seit langem auf frühere „commitments“, ,agreements“, ,agreed goals“ oder „pledges“ Bezug. Die Dokumente belegen daher nicht nur eine konsistente Praxis, sondern auch, daß der Praxis eine opinio iuris zugrundeliegt. Die Praxis ist bei der Auslegung einschlägiger Verträge, etwa des Sozialpakts, zu beachten. Die Annahme einer Rechtspflicht auch der Industriestaaten zur Zusammenarbeit fügt sich im übrigen zwanglos in das Programm des Sozialpaktes. Im General Comment No. 3 zum Sozialpakt (nature of States parties obligations) wird festgehalten: „[I]nternational cooperation for development ... is an obligation of all States. It is particularly incumbent upon those States which are in a position to assist 
auch immer dann, wenn sie - wie im Fall von extremer Armut - mit den Menschenrechten nicht zu vereinbaren ist. Dabei handelt es sich zunächst um eine Aufgabe der Staaten, die sich auf ihr Territorium bezieht. Über die Pflicht zur internationalen Zusammenarbeit bleibt die Aufgabe allerdings nicht auf das eigene Territorium beschränkt.

\section{Pflicht zur Beseitigung globaler sozialer Ungleichheit}

Wer annimmt, dass Staaten andere Staaten bei der Erfüllung der Pflichten aus Art. 11 des Sozialpakts unterstützen müssen, steht vor einer Herausforderung: Wie ist eine rechtliche Pflicht $\mathrm{zu}$ fassen, wenn doch praktisch alles unbestimmt ist: Art. 11 des Sozialpaktes gibt ein Ziel vor, lässt die Instrumente aber offen. Die internationale Schutzverantwortung trifft pauschal alle Staaten; Einzelbeiträge werden nicht abgegrenzt. Schließlich: Wenn der von Art. 11 des Sozialpakts geforderte Zustand weiter unerreicht bleibt, wer soll dafür verantwortlich sein?

Die Herausforderung ist nicht so dramatisch, wenn man die Anwendung physischen Zwangs (Krieg, Repressalie) nicht für ein zwingendes Merkmal des Pflichtbegriffs hält - und es scheint mir für das moderne Völkerrecht angebracht, diese Annahme aufzugeben. Dann ist zum einen festzuhalten: Seit 1990 werden die Felder der internationalen Zusammenarbeit näher definiert, durch die Generalversammlung oder durch hochrangige Gipfeltreffen. ${ }^{140} \mathrm{Zu}$ den Feldern gehören vor allem

others in this regard“ (para. 14). Ahnlich General Comment No. 15 (right to water) para. 30 und General Comment No. 19 (right to social security) para. 52. Menschenrechtliche Unterstützungspflichten bejahend auch S. I. Skogly/M. Gibney (Fn. 118); A. Sengupta Poverty Eradication (Fn. 118) 19; R. Hennessy Defining States' International Legal Obligations to Cooperate for the Achievement of Human Development: One Aspect of Operationalising a Human Rights-Based Approach to Development, in: M. Scheinin/M. Suksi (Hrsg.) Human Rights Development Yearbook 2002, $2005,71$.

140 Vgl. zunächst die Declaration on International Economic Cooperation 1990 (Fn. 120), International Development Strategy for the Fourth United Nations Development Decade (Fn. 120) sowie die dazu ergangenen Implementationsberichte des Generalsekretärs der Vereinten Nationen (A/46/505, 30 September 1991; A/47/397, 11 September 1992; A/49/328, 23 August 1994; A/51/270, 8 August 1996; A/53/301, 10 September 1998; A/54/389, 22 September 1999; A/55/209, 31 July 2000; A/57/216, 16 July 2002). Nach dem Monterrey Consensus 2002 erhielten die Bemühungen um eine Konkretisierung der internationalen Zusammenarbeit einen neuen Auftrieb. Der Monterrey Consensus definierte für die finanzielle Seite der „Entwicklung“ sechs wichtige Aktionsfelder: mobilizing domestic financial resources for development; mobilizing international resources for development: foreign direct investment and other private flows; international trade as an engine for development; increasing international financial and technical cooperation for development; external debt; enhancing coherence and consistency of the international monetary, financial and trading systems in support of development. Seit- 
die Entwicklungshilfe der Industrienationen, die Schuldenpolitik, die Politik der Weltbank und die Welthandelspolitik; Armutsbekämpfung hat dabei hohe Priorität. ${ }^{141}$ Zum anderen haben sich spezifische Durchsetzungsmechanismen entwickelt, die durchaus wirksam sein können. Dazu gehören Verhandlungen über benchmarking, Kontrollberichte, Konferenzen mit Zugang für NGOs, die Gelegenheit für „,naming“ und „shaming“ bieten. ${ }^{142}$ Zuletzt werden bei der Implementation von Vorhaben Kooperationspflichten weiter heruntergebrochen, für die Nehmerländer regelmäßig mehr als für Geberländer. Wichtige Instrumente sind

her beschäftigen sich (regionale bzw. thematisch begrenzte) Nachfolge-Konferenzen und hochrangige Treffen mit Fragen der Zusammenarbeit. In diese Gespräche sind nicht nur Staatenvertreter, sondern auch die internationalen Finanzinstitutionen (Weltbank, Internationaler Währungsfonds), die OECD, die WTO und einschlägige Organe der Vereinten Nationen eingebunden.

${ }^{141}$ Das Aktionsfeld „internationale Zusammenarbeit für die Entwicklung“ bezieht sich auch, aber nicht nur, auf die Höhe der Entwicklungshilfe (official development assistance). Das Aktionsfeld bezieht sich ganz allgemein auf die Architektur der Entwicklungshilfe. Der Grundgedanke des Monterrey Consensus ist die Schaffung einer neuen „Partnerschaft" auf der Basis von „wechselseitiger Verantwortlichkeit“ (partnership; mutual accountability). Welche Inhalte diese Schlagworte in der Praxis haben können, wird seither diskutiert, im Rahmen des Monterrey Prozesses und im Rahmen der Menschenrechtsorgane der Vereinten Nationen. Vgl. dazu die Implementationsberichte des Generalsekretärs (A/58/216, 5 August 2003, para. 101; A/59/822, 1 June 2005, para. 24; A/61/253, 8 August 2006, para. 32; A/62/217, 10 August 2007, para. 76) und die Arbeiten der Arbeitsgruppe zum Recht auf Entwicklung, die sich spezifisch mit der Bewertung von Entwicklungs-Zusammenarbeit befassen (jüngst: A/HRC/4/47, 14 March 2007; E/CN.4/2006/26, 22 February 2006).

${ }_{142}$ Auf dem Gebiet der Entwicklungshilfe (official development aid) etwa beziehen sich die Verhandlungen auf einen Zeitplan für die Erhöhung der Zahlungen und die Steigerung der Effektivität von Entwicklungshilfe. Mit Blick auf Effektivitätsfragen einigten sich die Staatenvertreter auf fünf inhaltliche Anliegen (ownership of development strategies by the developing countries; alignment of donor support with these strategies; harmonization of donor actions; managing for result; mutual accountability), 50 konkrete Verpflichtungen (commitments) und 12 Indikatoren zur Überwachung. Vgl. hierzu Paris Declaration on Aid Effectiveness 2005, abrufbar unter: http://www.oecd.org/dac/effectiveness). Anfang September 2008 tagte das dritte High-Level Forum on Aid Effectiveness in Accra, Ghana, bei dem die Entwicklungspolitik der Industriestaaten zum Teil heftig kritisiert wurde, weil Richtgrößen verfehlt wurden (Zugang zu den Dokumenten über: http://www.oecd.org/dac/effectiveness/accrahlf). Die Kritik konnte sich auf den jüngsten Kontrollbericht stützen: 2008 Survey on monitoring the Paris Declaration: Effective aid by 2010? What it will take, 2008. Alle Aktivitäten im Gefolge des Monterrey Consensus 2002 werden Ende November/Anfang Dezember 2008 bei der Monterrey-Nachfolgekonferenz in Doha, Qatar erneut gebündelt. Auf diese Konferenz hat sich die Generalversammlung der Vereinten Nationen im Dezember 2007 festgelegt (A/RES/62/187, 19 December 2007). 
dabei die Poverty Reduction Strategy Papers, an denen die Weltbank ihre Mittelvergabe ausrichtet. ${ }^{143}$

Meine zehnte These ist: Die Aktivitäten auf der internationalen Ebene haben eine Dynamik entfaltet, die mögliche Einwände gegen die Annahme einer inhaltlich bestimmten Rechtspflicht für Industrienationen zur globalen Armutsbekämpfung mindestens abschwächen. Die fortdauernden Verhandlungen erzeugen einen beträchtlichen Druck, Zusagen zu machen und einzuhalten. Für Interessierte wird außerdem sichtbar, wer Einigungen blockiert.

\section{Zusammenfassung}

Soziale Ungleichheit interessiert die Medien, die Politik, die Soziologie, die politische Philosophie. Das deutsche Recht hat dafür wenig Sensorien entwickelt. Wenn soziale Ungleichheit sozialrechtlich nicht bearbeitet oder vergrößert wird, wird dies erst dann zum Problem, wenn die Menschenwürde verletzt ist oder - im Versicherungssystem - wenn die gewährten Leistungen so niedrig sind, dass sie die abverlangten Beiträge nicht rechtfertigen. Der Aspekt des Verdienstes oder des Zufalls spielt keine Rolle. Der Gleichheitssatz ist nur am Rande bedeutsam. Dass konsequent eingeforderte rechtliche Gleichheit auf soziale Ungleichheit durchschlagen kann, belegen meine Untersuchungen zum Status der Angehörigen der EU-Anwerbeländer. Die Kommission der Europäischen Gemeinschaften hatte den Mitgliedstaaten gelegentlich vorgeworfen, dass sie mit ihrer Migrationspolitik soziale Ungleichheit festigen würden. Der Vorwurf trifft die Bundesrepublik zu Recht. Dem Völkerrecht ist zu entnehmen, dass Solidarität „flüssig“ geworden ist. Es ist heute problematisch, der extremen Armut in den Entwicklungsländern keine rechtliche Bedeutung beizumessen. Aus einer etwas anderen Perspektive: Das Völkerrecht und das staatliche Recht nehmen soziale Ungleichheit in weitem Umfang in Kauf. Für das klassische Völkerrecht war dies überhaupt die Voraussetzung für die Einigung auf eine gemeinsame Ordnung. Für das deutsche Recht ist dies zum einen auf die Freiheitsrechte zurückzuführen - das Grundgesetz hat eine libertäre Ausrichtung -, zum anderen auf das Gewicht, das die Verfassung insoweit der Politik zubilligt. Hoffnung und Lethargie verleihen solchen ungleichen Ordnungen dennoch Stabilität.

143 Zur Politik der Weltbank näher: Operations Policy and Country Services, World Bank Conditionality in Development Policy Lending, 2007; World Bank Meeting the Challenges of Global Development. A Long-Term Strategic Exercise, 2007. 
Anhang: Tabellen

\begin{tabular}{|c|c|c|c|c|c|c|c|c|c|c|c|c|c|c|c|}
\hline હે̊ & 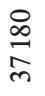 & 守 & $\begin{array}{l}\stackrel{\sim}{~} \\
\text { } \\
\infty \\
+\end{array}$ & $\begin{array}{l}\stackrel{8}{ } \\
\stackrel{n}{\infty} \\
\infty \\
m\end{array}$ & $\begin{array}{l}\text { \& } \\
\text { ஓे } \\
\text { m }\end{array}$ & $\begin{array}{l}\stackrel{\infty}{\infty} \\
\stackrel{+}{+}\end{array}$ & 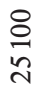 & $\begin{array}{l}\stackrel{\rho}{m} \\
\stackrel{m}{F} \\
\vec{\gamma}\end{array}$ & $\begin{array}{l}\stackrel{ }{m} \\
\stackrel{m}{m}\end{array}$ & $\frac{P}{\frac{1}{6}}$ & $\begin{array}{l}0 \\
\text { ర0 } \\
2 \\
\text { లn }\end{array}$ & 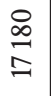 & $\begin{array}{l}8 \\
8 \\
+ \\
\sim \\
\sim\end{array}$ & $\begin{array}{l}\text { 으 } \\
\text { ò } \\
\text { f }\end{array}$ & $\underset{\substack{\infty \\
\text { d }}}{\stackrel{P}{+}}$ \\
\hline હેّ & $\begin{array}{l}\text { 응 } \\
\text { 웅 } \\
\text { N }\end{array}$ & $\begin{array}{l}8 \\
\infty \\
\tilde{n} \\
\sim\end{array}$ & $\begin{array}{l}0 \\
\infty \\
\infty \\
m\end{array}$ & 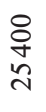 & 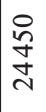 & $\begin{array}{l}0 \\
i n \\
\end{array}$ & $\begin{array}{l}\stackrel{\ominus}{\mathscr{J}} \\
\stackrel{\text { I }}{\Xi}\end{array}$ & $\begin{array}{l}\stackrel{8}{0} \\
\stackrel{\sim}{\sim}\end{array}$ & 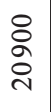 & 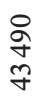 & $\begin{array}{l}8 \\
\infty \\
0 \\
0 \\
\sim \\
\sim\end{array}$ & $\begin{array}{l}8 \\
0 \\
=\end{array}$ & 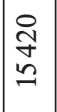 & $\begin{array}{l}\stackrel{0}{\infty} \\
\infty \\
\infty \\
\stackrel{\infty}{N}\end{array}$ & 尽 \\
\hline$\stackrel{2}{\sigma}$ & $\begin{array}{l}\stackrel{\circ}{\stackrel{2}{n}} \\
\stackrel{\sim}{N}\end{array}$ & $\begin{array}{l}8 \\
\text { ¿ } \\
\stackrel{0}{0} \\
\text { in }\end{array}$ & 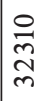 & 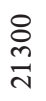 & \begin{tabular}{l}
$\curvearrowright$ \\
$\infty$ \\
\multirow{2}{n}{}
\end{tabular} & 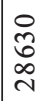 & $\begin{array}{l}8 \\
\text { ¿ } \\
\end{array}$ & 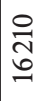 & 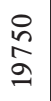 & $\begin{array}{l}\stackrel{0}{0} \\
\stackrel{0}{0} \\
m\end{array}$ & $\begin{array}{l}\stackrel{0}{0} \\
\tilde{b} \\
\sim \\
\end{array}$ & 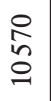 & 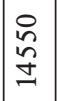 & 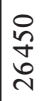 & $\begin{array}{l}\stackrel{ }{\circ} \\
\stackrel{2}{2}\end{array}$ \\
\hline જิ & $\stackrel{\circ}{\stackrel{\infty}{\circ}}$ & $\begin{array}{l}\stackrel{2}{\infty} \\
\stackrel{2}{2} \\
\infty\end{array}$ & $\frac{8}{\stackrel{一}{\sim}}$ & \begin{tabular}{l}
$\stackrel{0}{\infty}$ \\
\multirow{\sim}{\sim}{} \\
$\sim$
\end{tabular} & $\underset{\text { O্+ }}{\stackrel{\text { }}{\sim}}$ & 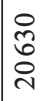 & $\begin{array}{l}\text { ㅁ } \\
\text { જ̆ }\end{array}$ & $\begin{array}{l}\stackrel{8}{0} \\
\stackrel{0}{\beth}\end{array}$ & $\begin{array}{l}\searrow \\
\searrow \\
=\end{array}$ & 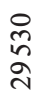 & $\begin{array}{l}\stackrel{1}{1} \\
\infty \\
\infty \\
-1\end{array}$ & ஓ & $\begin{array}{l}\stackrel{\circ}{\infty} \\
= \\
=\end{array}$ & 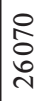 & 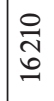 \\
\hline $\begin{array}{l}10 \\
2 \\
2\end{array}$ & $\frac{\circ}{\sigma}$ & 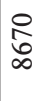 & 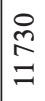 & 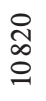 & 8̊ & 을 & 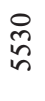 & 命 & $\stackrel{ }{\stackrel{2}{ }}$ & 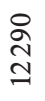 & \begin{tabular}{l}
8 \\
\multirow{2}{n}{} \\
\multirow{2}{*}{}
\end{tabular} & 요 & 导 & 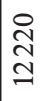 & 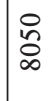 \\
\hline $\begin{array}{l}\stackrel{\circ}{\circ} \\
\stackrel{2}{2}\end{array}$ & 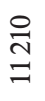 & $\begin{array}{l}\stackrel{8}{2} \\
m\end{array}$ & ৪ & $\begin{array}{l}\stackrel{\text { త }}{=} \\
=\end{array}$ & 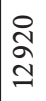 & $\begin{array}{l}\stackrel{8}{0} \\
\stackrel{0}{1}\end{array}$ & $\stackrel{ }{\stackrel{2}{ }}$ & $\frac{0}{6}$ & ஜे & 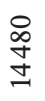 & $\begin{array}{l}0 \\
6 \\
m \\
m\end{array}$ & $\frac{\stackrel{\circ}{0}}{m}$ & $\begin{array}{l}8 \\
8 \\
0\end{array}$ & 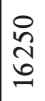 & 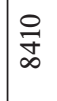 \\
\hline$\frac{1}{2}$ & $\underset{n}{\stackrel{f}{f}}$ & 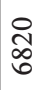 & ণิ & $\frac{\text { O }}{6}$ & $\begin{array}{l}8 \\
\infty \\
0 \\
0\end{array}$ & 음 & 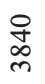 & $\begin{array}{l}\stackrel{8}{0} \\
\text { m }\end{array}$ & $\stackrel{\circ}{\mathscr{n}}$ & $\underset{ }{\stackrel{ }{ }}$ & $\frac{2}{2}$ & $\begin{array}{l}\stackrel{2}{\circ} \\
\stackrel{\sim}{\sim} \\
\end{array}$ & $\frac{\infty}{m}$ & 을 & $\underset{\text { }}{\stackrel{ }{f}}$ \\
\hline 옹 & 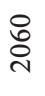 & $\stackrel{\infty}{\stackrel{\infty}{N}}$ & : & 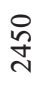 & 을 & : & 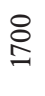 & 욤 & ণ্ণి & 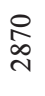 & $\frac{8}{\stackrel{8}{N}}$ & $\stackrel{8}{\infty}$ & $\underset{8}{\stackrel{0}{0}}$ & 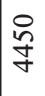 & : \\
\hline ஜ̊ & 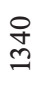 & $\stackrel{0}{\stackrel{\infty}{-1}}$ & : & $\stackrel{\circ}{\infty}$ & ஜ̊ & : & 옹 & ○ & 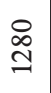 & 음 & $\stackrel{ }{\equiv}$ & 음 & 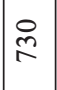 & 으 & : \\
\hline 导 & $\stackrel{2}{2}$ & $\stackrel{\infty}{2}$ & $\stackrel{m}{2}$ & 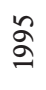 & $\stackrel{\infty}{2}$ & $\begin{array}{l}\infty \\
\stackrel{2}{2}\end{array}$ & $\begin{array}{l}\bar{\infty} \\
\stackrel{2}{2}\end{array}$ & $\stackrel{m}{\stackrel{2}{a}}$ & 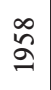 & $\stackrel{\infty}{\curvearrowleft}$ & $\begin{array}{l}\infty \\
2 \\
2\end{array}$ & \begin{tabular}{l}
$\circ$ \\
$\infty$ \\
\hdashline
\end{tabular} & $\begin{array}{l}0 \\
\infty \\
2\end{array}$ & ฉ̊ & $\frac{m}{a}$ \\
\hline 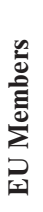 & 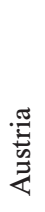 & 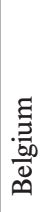 & 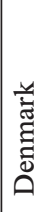 & 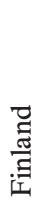 & 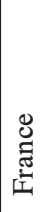 & 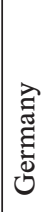 & 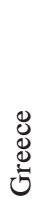 & 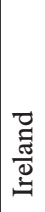 & 齐 & 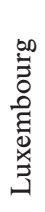 & 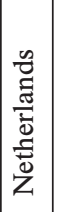 & 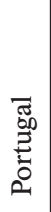 & 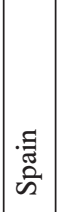 & 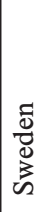 & $\stackrel{凶}{\supset}$ \\
\hline
\end{tabular}


Tabelle 2: Schulabschlïsse der Einwanderer 1986-2006 (Vergleichsgruppe: Deutsche)

\begin{tabular}{|l|r|r|r|r|r|r|r|r|r|}
\hline \multirow{2}{*}{$\begin{array}{l}\text { Staatsange- } \\
\text { hörigkeit }\end{array}$} & \multicolumn{3}{|c|}{ Realschule } & \multicolumn{3}{|c|}{ Fach/Hochschulreife } & \multicolumn{3}{|c|}{ Kein Abschluß } \\
\cline { 2 - 10 } & $\mathbf{1 9 8 6}$ & $\mathbf{2 0 0 6}$ & $+/-$ & $\mathbf{1 9 8 6}$ & $\mathbf{2 0 0 6}$ & $+/-$ & $\mathbf{1 9 8 6}$ & $\mathbf{2 0 0 6}$ & $+/-$ \\
\hline Deutsche & 22,2 & 30,2 & $+8,0$ & 15,6 & 26,2 & $+10,6$ & 3,3 & 1,1 & $-2,2$ \\
\hline $\begin{array}{l}\text { Anwerbe- } \\
\text { länder EU }\end{array}$ & 4,1 & 11,5 & $+7,4$ & 2,6 & 10,1 & $+7,5$ & 34,2 & 12,5 & $-21,7$ \\
\hline $\begin{array}{l}\text { Ex-Jugos- } \\
\text { lawien }\end{array}$ & 2,4 & 9,8 & $+7,4$ & 1,8 & 5,5 & $+3,7$ & 28,7 & 13,6 & $-15,1$ \\
\hline Türkei & 2,4 & 7,5 & $+5,1$ & 1,1 & 5,2 & $+4,1$ & 37,3 & 17,5 & $-19,2$ \\
\hline
\end{tabular}

Quelle: SOEP, eigene Berechnung (Angaben in Prozent der jeweiligen Teilgruppe; Änderung in Prozentpunkten)

Tabelle 3: Fehlen einer beruflichen Ausbildung 1986-2006 (Vergleichsgruppe: Deutsche)

\begin{tabular}{|l|c|c|c|}
\hline \multirow{2}{*}{ Staatsangehörigkeit } & \multicolumn{3}{|c|}{ Kein AbschluB } \\
\cline { 2 - 4 } & $\mathbf{1 9 8 6}$ & $\mathbf{2 0 0 6}$ & $+/-$ \\
\hline Deutsche & 25,6 & 14,9 & $-10,7$ \\
\hline Anwerbeländer EU & 64,7 & 49,4 & $-15,3$ \\
\hline Ex-Jugoslawien & 50,8 & 41,3 & $-9,5$ \\
\hline Türkei & 69,0 & 62,0 & $-7,0$ \\
\hline
\end{tabular}

Quelle: SOEP, eigene Berechnung (Angaben in Prozent der jeweiligen Teilgruppe; Änderung in Prozentpunkten) 
Tabelle 4: Brutto-Erwerbseinkommen, Durchschnittseinkommen 1986

\begin{tabular}{|l|r|r|r|r|}
\hline Staatsangehörigkeit & \multirow{2}{*}{ DEU } & EU-A & Ex-JUG & TUR \\
\cline { 1 - 4 } Quintile & 690 & 869 & 920 & 869 \\
\hline 20 & 1176 & 1176 & 1176 & 1125 \\
\hline 60 & 1483 & 1329 & 1432 & 1329 \\
\hline 80 & 1954 & 1537 & 1694 & 1585 \\
\hline 100 & 16569 & 6678 & 4910 & 4602 \\
\hline Durchschnitt & 1440 & 1245 & 1315 & 1238 \\
\hline
\end{tabular}

Quelle: SOEP, eigene Berechnung (Angaben in Euro); DEU = Deutschland; $\mathrm{EU}-\mathrm{A}=$ Anwerbeländer EU; Ex-JUG = Ex-Jugoslawien; TUR = Türkei

Tabelle 5: Brutto-Erwerbseinkommen, Durchschnittseinkommen 1996

\begin{tabular}{|l|c|c|c|c|}
\cline { 1 - 2 } Staatsangehörigkeit & \multirow{2}{*}{ DEU } & EU-A & Ex-JUG & TUR \\
\cline { 1 - 3 } Quintile & 1023 & 1192 & 1023 & 1023 \\
\hline 20 & 1534 & 1636 & 1483 & 1554 \\
\hline 60 & 1951 & 1943 & 1841 & 1843 \\
\hline 80 & 2556 & 2301 & 2199 & 2282 \\
\hline 100 & 12782 & 7158 & 6198 & 7669 \\
\hline Durchschnitt & 1895 & 1817 & 1680 & 1690 \\
\hline
\end{tabular}

Quelle: SOEP, eigene Berechnung (Angaben in Euro); DEU = Deutschland; $\mathrm{EU}-\mathrm{A}=$ Anwerbeländer EU; Ex-JUG = Ex-Jugoslawien; TUR = Türkei 
Tabelle 6: Brutto-Erwerbseinkommen, Durchschnittseinkommen 2006

\begin{tabular}{|l|r|r|r|r|}
\hline Staatsangehörigkeit & \multirow{2}{*}{ DEU } & EU-A & Ex-JUG & TUR \\
\cline { 1 - 4 } Quintile & 926 & 1000 & 934 & 666 \\
\hline 20 & 1700 & 1800 & 1524 & 1600 \\
\hline 40 & 2400 & 2400 & 2200 & 2200 \\
\hline 80 & 3300 & 3050 & 2683 & 2800 \\
\hline 100 & 20000 & 10278 & 8800 & 7500 \\
\hline Durchschnitt & 2312 & 2199 & 1972 & 1897 \\
\hline
\end{tabular}

Quelle: SOEP, eigene Berechnung (Angaben in Euro); DEU = Deutschland; $\mathrm{EU}-\mathrm{A}=$ Anwerbeländer EU; Ex-JUG = Ex-Jugoslawien; TUR = Türkei

Tabelle 7: Anteil der Arbeiter bzw. der Angestellten an den Erwerbstätigen 1986-2006

(Vergleichsgruppe: Deutsche)

\begin{tabular}{|l|r|r|r|r|r|c|}
\hline \multirow{2}{*}{ Staatsangehörigkeit } & \multicolumn{3}{|c|}{ Anteil der Arbeiter } & \multicolumn{3}{c|}{ Anteil der Angestellten } \\
\cline { 2 - 7 } & $\mathbf{1 9 8 6}$ & $\mathbf{2 0 0 6}$ & \multicolumn{1}{|c|}{+ - } & $\mathbf{1 9 8 6}$ & $\mathbf{2 0 0 6}$ & $+/-$ \\
\hline Deutsche & 31,8 & 24,5 & $-7,3$ & 40,2 & 48,7 & $+8,5$ \\
\hline Anwerbeländer EU & 78,0 & 54,2 & $-23,8$ & 4,0 & 31,0 & $+22,4$ \\
\hline Ex-Jugoslawien & 81,0 & 54,7 & $-26,3$ & 10,0 & 36,0 & $+26,0$ \\
\hline Türkei & 88,1 & 71,0 & $-17,1$ & 0,4 & 15,8 & $+11,8$ \\
\hline
\end{tabular}

Quelle: SOEP, eigene Berechnung (Angaben in Prozent der jeweiligen Teilgruppe; Änderung in Prozentpunkten) 
Leitsätze der 1. Berichterstatterin über:

\section{Soziale Gleichheit: Voraussetzung oder Aufgabe der Verfassung?}

\section{Warum Gleichheit?}

1. Soziale Gleichheit ist weder Voraussetzung noch Aufgabe einer Verfassung. Das gilt für die Verfassungsordnung des Grundgesetzes, für das Gemeinschaftsrecht und das Völkerrecht. Keine der Ordnungen beruht auf einem egalitären Verteilungsprinzip. Moderne Verfassungen haben gerade die Funktion einer Klammer für Differenzierungen und, darin eingeschlossen, soziale Ungleichheiten.

\section{Soziale Ungleichheit und Grundgesetz}

Gesundheit und Ungleichheit

2. Im Gesundheitsbereich kann die Vergrößerung von sozialer Ungleichheit nur ausnahmsweise gleichheitsrechtlich angegriffen werden. Der Maßstab des Gleichheitssatzes ist zum Teil zu grob, zum Teil ungeeignet.

3. Das Unterschreiten des Existenzminimums ist grundrechtlich problematisch. Diese Form von sozialer Ungleichheit ist nicht hinzunehmen. Es zeichnet sich allerdings ein breiter Konsens dahin ab, den Mindeststandard deutlich unterhalb der Standards des BSHG anzusetzen. Unter Art. 1 Abs. 1 $G G$ bezieht er sich danach primär auf die Dimensionen Nahrung, Kleidung, Obdach, unter Art. 2 Abs. 2 S. 1 GG auf eine Grundversorgung im Bereich der Gesundheit. Damit nähert sich der Standard des GG dem völkerrechtlichen Standard des Art. 11 des Sozialpakts der Vereinten Nationen.

\section{Alterssicherung und Ungleichheit}

4. Aus Art. 14 Abs. 1 oder Art. 2 Abs. 1 GG folgen keine schlagkräftigen Einwände gegen eine Gestaltung der Alterssicherung, die zu größeren Einkommensunterschieden führt. Die Erzeugung von sozialer Ungleichheit ist insoweit unproblematisch. 


\section{Grundsicherung und Ungleichheit}

5. Das SGB II hat den Menschenwürde-Standard des BSHG deutlich abgesenkt. Die Sozialgerichte sind verunsichert und bemüht, dem durch verfassungskonforme Auslegung gegenzusteuern. Sie scheuen aber davor zurück, mit dem Gesetzgeber in einem Gerichtsverfahren über den genauen Verlauf der denkbar untersten Grenze des Existenzminimums zu streiten.

6. Die Sozialpolitik der letzten Jahre führt gerade für Menschen, die Arbeitslosengeld II oder Sozialhilfe beziehen, zu multipler Exklusion. Es gibt kein Gerichtsverfahren, in dem diese spezifische Form der Exklusion angemessen thematisiert werden könnte. Es gibt deshalb keine Instanz, die die Einhaltung der Menschenwürde-Garantie verlässlich prüfen kann.

\section{Europäisierung der Gleichheit}

7. Aus der Sicht des Gemeinschaftsrechts ist soziale Ungleichheit problematisch, wenn sie ihre Basis in rechtlicher Ungleichheit hat. Die Rechtsprechung des EuGH zu den „sozialen Vergünstigungen“ gibt der Freizügigkeit eine starke sozialrechtliche Komponente. Social citizenship ist Teil der Unionsbürgerschaft. Das hat explizit das Ziel, mehr soziale Gleichheit herzustellen.

8. Es gibt einen plausiblen Zusammenhang zwischen der strikten Inklusionspolitik der Gemeinschaft und der Lage der Arbeitskräfte aus den EUAnwerbeländern. Ihre Lage unterscheidet sich noch immer von der der Deutschen. Sie haben sich in ihren Ressourcen den Deutschen indes mehr angenähert als die Angehörigen der anderen Anwerbeländer. Anders gewendet: Die Aufgabe der Gemeinschaft bezieht sich auf rechtliche Gleichheit. Rechtliche Gleichheit bewirkt hier jedoch zugleich mehr soziale Gleichheit.

\section{Globale soziale Ungleichheit: Verantwortung für die Welt}

9. Im globalen Zusammenhang wird soziale Ungleichheit zu einer Aufgabe nur dann, aber auch immer dann, wenn sie - wie im Fall von extremer Armut - mit den Menschenrechten nicht zu vereinbaren ist. Dabei handelt es sich zunächst um eine Aufgabe der Staaten, die sich auf ihr Territorium bezieht. Über die Pflicht zur internationalen Zusammenarbeit bleibt die Aufgabe allerdings nicht auf das eigene Territorium beschränkt.

10. Die Aktivitäten auf der internationalen Ebene haben eine Dynamik entfaltet, die mögliche Einwände gegen die Annahme einer inhaltlich bestimmten Rechtspflicht für Industrienationen zur globalen Armutsbekämpfung mindestens abschwächen. Die fortdauernden Verhandlungen erzeugen einen beträchtlichen Druck, Zusagen zu machen und einzuhalten. Für Interessierte wird außerdem sichtbar, wer Einigungen blockiert. 
Zweiter Beratungsgegenstand:

\section{Soziale Gleichheit - Voraussetzung oder Aufgabe der Verfassung?}

2. Bericht von Professor Dr. Peter Axer, Trier

Inhalt

Seite

I. Soziale Ungleichheit als Problem . . . . . . . . . . . . . 178

1. Formen und Felder sozialer Ungleichheit . . . . . . . . 180

2. Abbau sozialer Ungleichheiten und Herstellung sozialer

Gleichheit: der ökonomische Bezug des Sozialen . . . . 183

II. Soziale Gleichheit als Aufgabe von Staat und Gesellschaft 185

III. Sinn und Zweck sozialer Gleichheit . . . . . . . . . . . . 186

1. Soziale Gleichheit als eine Voraussetzung staatlicher Einheit . . . . . . . . . . . . . 186

2. Soziale Gleichheit als eine Voraussetzung der

Grundrechtsausübung . . . . . . . . . . . . . 190

IV. Inhalt und Umfang sozialer Gleichheit . . . . . . . . . . . 192

1. Soziale Gleichheit und Rechtsstaatsprinzip . . . . . . . 193

2. Soziale Gleichheit als Thema des sozialen Staatsziels . . 194

3. Soziale Gleichheit durch soziale Sicherheit . . . . . . 195

a) Gewährleistung des Existenzminimums . . . . . . 196

b) Soziale Vorsorge . . . . . . . . . . . . 202

4. Soziale Gleichheit durch sozialen Ausgleich . . . . . . 207

5. Soziale Gleichheit als Chancengleichheit . . . . . . . 208

6. Soziale Gleichheit und Rechtsgleichheit . . . . . . . . . 211

7. Soziale Gleichheit und demokratischer Prozess . . . . . 214

V. Soziale Gleichheit als Thema der Verfassung . . . . . . . 215 


\section{Soziale Ungleichheit als Problem}

„Weil Du arm bist, musst Du früher sterben“, lautet eine alte volkstümliche Redewendung. ${ }^{1}$ Diese besitzt trotz einer auch in Deutschland insgesamt gestiegenen Lebenserwartung nach wie vor Aktualität und Berechtigung. ${ }^{2}$ Immer noch beeinflussen die jeweiligen Lebensumstände Morbidität und Mortalität: Je höher das Einkommen, desto eher besteht Aussicht auf ein langes und gesundes Leben. ${ }^{3}$

Armut dagegen vermindert und zerstört in der statistisch gesehen ${ }^{4}$ ohnehin schon kürzeren Lebenszeit reale Freiheit. ${ }^{5}$ Wer arm ist, kann nur schwerlich - wenn überhaupt - Eigentum bilden, Unternehmen gründen oder am kulturellen Leben teilnehmen. Da die Zahl der Menschen, die nach dem relativen Armutsbegriff in Armut leben oder von ihr bedroht sind, nach wie vor hoch ist, ${ }^{6}$ sich die Schere zwischen arm

${ }^{1} \mathrm{Zu}$ Herkunft und Bedeutung der Redewendung, die auch den Titel eines verfilmten Romans von Hans G. Kernmayr bildet: H. Schmitthenner/H.-J.Urban Globaler Markt und sozialer Staat - ein unauflösbarer Gegensatz?, in: C. Butterwegge/M. Kutscha/ S. Berghahn (Hrsg.) Herrschaft des Marktes - Abschied vom Staat, 1999, 45 (61); s. auch: A. Oppolzer Wenn Du arm bist, musst Du früher sterben, 1986.

2 I. Ebsen Armut und Gesundheit, SDSRV 56 (2007), 133 (135ff.); Th. Lampert/ L. E. Kroll/A. Dunkelberg Soziale Ungleichheiten der Lebenserwartung in Deutschland, APuZ 2007, Heft 42, 11 ff.; Th. Lampert/A. Mielck Gesundheit und soziale Ungleichheit, G+G Wissenschaft, 2/2008, 7 ff.; Th. Lampert Soziale Ungleichheiten der Gesundheitschancen und Krankheitsrisiken, KV 2007, 23 ff.; Lebenslagen in Deutschland - Dritter Armuts- und Reichtumsbericht, BT-Drucks. 16/9915, $82 \mathrm{ff}$.

${ }^{3}$ Lampert/Kroll/Dunkelberg Soziale Ungleichheiten (Fn. 2), 11 (12ff.). - Zur Bedeutung der Arbeitsbedingungen und Lebensumstände für das Krankheitsrisiko allgemein: G. Bäcker/G. Naegele/R. Bispinck/K. Hofemann/J. Neubauer Sozialpolitik und soziale Lage in Deutschland, Bd. 2, 4. Aufl. 2008, 43 ff., $91 \mathrm{ff}$.

${ }_{4}$ Nach dem dritten Armuts- und Reichtumsbericht (Fn. 2), 83, halbiert monetäre Armut in etwa die Chance auf einen sehr guten oder guten Gesundheitszustand; s. auch Lampert/Mielck Gesundheit (Fn. 2), $10 \mathrm{f}$., wonach die mittlere Lebenserwartung von Männern, die der niedrigsten Einkommensgruppe zugehören, 70,1 Jahre beträgt, die von Männern, die der höchsten Einkommensgruppe zugehören, 80,9 Jahre; ein entsprechendes Bild zeigt sich bei Frauen (76,9 Jahre bzw. 85,3 Jahre).

${ }^{5}$ Vgl. P. Kirchhof Armut und Freiheit, FS Zacher, 1998, 323. - Zur Bedeutung und zum Verhältnis von realer und rechtlicher Freiheit allgemein: W. Krebs Rechtliche und reale Freiheit, in: D. Merten/H.-J. Papier (Hrsg.) Handbuch der Grundrechte, Bd. II, 2006, § 31 Rn. $1 \mathrm{ff}$.

6 Zum Begriff „Armut“ und dessen Bestimmung vgl. etwa: G. Bäcker Altersarmut als soziales Problem der Zukunft?, DRV 2008, 357 ff.; R. Hauser Das Maß der Armut: Armutsgrenzen im sozialstaatlichen Kontext. Der sozialstaatliche Diskurs, in: E.-U. Huster/J. Boeckh/H. Mogge-Grotjahn (Hrsg.) Handbuch Armut und soziale Ausgrenzung, 2008, 94 ff. - Für die Bestimmung, wann Armut vorliegt, wird zumeist ein relativer Armutsbegriff aufgrund einer Armutsrisikoquote zugrunde gelegt. Danach gilt als arm, 
und reich, trotz geringerer Lohnspreizung im Jahre 2006, ${ }^{7}$ seit Mitte der neunziger Jahre weit geöffnet hat ${ }^{8}$ und die Mittelschicht schrumpft ${ }^{9}$, driften die Lebensverhältnisse auseinander ${ }^{10}$ und macht sich Verunsicherung gerade in der Mitte der Gesellschaft breit.1 ${ }^{11}$ Soziale Ungleichheit wird in immer stärkerem Maße wahrgenommen ${ }^{12}$ und als nicht mehr hinnehmbar empfunden: Nicht einmal ein Fünftel der Deutschen glaubt heute, dass es in der Volkswirtschaft gerecht zugehe;13 knapp $70 \%$ der Deutschen sind der Ansicht, dass in den letzten Jahren soziale Gerechtigkeit abgenommen habe. ${ }^{14}$ Vor diesem Hintergrund besteht gerade in einer Gesellschaft wie der deutschen, in der der Sozialstaat und der Gedanke sozialer Gerechtigkeit tief verwurzelt sind und geradezu

wer weniger als $60 \%$ des Mittelwertes aller Einkommen zur Verfügung hat. Absolute oder primäre Armut liegt dagegen vor, wenn nicht genügend Mittel zum physischen Überleben vorhanden sind. Dazu und zum tatsächlichen Umfang der Armut bis zum Jahre 2005 vgl. den dritten Armuts- und Reichtumsbericht (Fn. 2), 16f., 37 ff. - Nach neueren Berechnungen soll sich die Zahl der Armutsgefährdeten im Jahre 2006 verringert haben, allerdings waren immer noch $16,5 \%$ der Bevölkerung armutsgefährdet (F.A.Z. vom 30. 7. 2008, 10).

${ }^{7}$ Handelsblatt v. 17. 9. 2008, 3; genaue Analyse bei K. Brenke Jahrzehntelanger Trend zunehmender Lohnspreizung gestoppt, DIW Wochenbericht 2008, $567 \mathrm{ff}$.

8 Vgl. dazu den dritten Armuts- und Reichtumsbericht (Fn. 2), 36; G. Bosch/T. Kalina/C. Weinkopf Niedriglohnbeschäftigte auf der Verliererseite, WSI Mitteilungen 8/2008, 423 ff.; B. Taffertshöfer Die Kluft wird größer, SZ v. 27. 8. 2008, 26; P. Krohn Wer gewinnt den Umverteilungskampf, F.A.Z. v. 20. 8. 2008, 16; s. auch: O. Groh-Samberg Die Armut der deutschen Klassengesellschaft, KV 2007, 18 ff.; Lampert Soziale Ungleichheiten (Fn. 2), 23f.; H.-J. Marcus Armut in Deutschland, SDSRV 56 (2007), 7 ff.; G. Trabert Armut und Gesundheit, KV 2007, 12 (12f.).

9 P. Krohn Die Mittelschicht schrumpft, F.A.Z. vom 26. 7. 2008, 10; s. auch: N. Burzan Die Absteiger: Angst und Verunsicherung in der Mitte der Gesellschaft, ApuZ 2008, Heft 33-34, 6ff.; M. M. Grabka/J. R. Frick Schrumpfende Mittelschicht - Anzeichen einer dauerhaften Polarisierung der verfügbaren Einkommen?, DIW Wochenbericht 2008, $101 \mathrm{ff}$., wonach die Mittelschicht in den Jahren 2000 bis 2006 von $62 \%$ auf $54 \%$ der Bevölkerung geschrumpft ist.

${ }^{10} \mathrm{Vgl}$. dazu S. Hradil Warum werden die meisten entwickelten Gesellschaften wieder ungleicher?, in: P. Windolf (Hrsg.) Finanzmarktkapitalismus, 2005, $460 \mathrm{ff}$.

${ }^{11}$ Dazu etwa: $K$. Dörre Armut, Abstieg, Unsicherheit: Die soziale Frage am Beginn des 21. Jahrhunderts, ApuZ 2008, Heft 33-34, 3 ff.; Burzan Angst und Verunsicherung in der Mitte (Fn. 9), $6 \mathrm{ff.}$; B. Vogel Die Staatsbedürftigkeit der Gesellschaft, 2007, $71 \mathrm{ff}$.

${ }^{12}$ Zur Wahrnehmung von Armut und Reichtum jüngst: P. Sachweh Sind Armut und Reichtum ein Problem?, SF 2008, $214 \mathrm{ff}$.

${ }^{13} \mathrm{Vgl}$. U. J. Heuser Ungleichheit ist nicht immer schlecht ..., Die Zeit vom 24. 1. 2008, 23.

${ }^{14}$ T. Petersen Die gefühlte Ungerechtigkeit, F.A.Z. vom 23. 7. $2008,5$. 
mythischen Charakter besitzen, ${ }^{15}$ die Gefahr dauerhafter, den sozialen Frieden gefährdender Spannungen und Spaltung. ${ }^{16}$

\section{Formen und Felder sozialer Ungleichheit}

„Kinderarmut“, „Altersarmut“, „Zwei-Drittel-Gesellschaft“, „ZweiKlassen-Medizin“, „Hartz IV“, „Rente mit 67“ oder „unterschiedliche Lebensverhältnisse in Ost und West" sind nur einige der aktuellen Begriffe und Themen, an denen sich die Diskussion über soziale Ungleichheit verbunden mit der Forderung nach sozialer Gerechtigkeit entzündet. „Soziale Gerechtigkeit"17 erscheint inzwischen im politischen Sprachgebrauch als Gerechtigkeit schlechthin ${ }^{18}$ und wird eingefordert, um als ungerecht empfundene Ungleichheiten zu korrigieren, gilt doch die gleichmäßige Verteilung von Chancen und materiellen Gütern häufig als das wesentliche Gebot sozialer Gerechtigkeit. Obwohl der inflationär verwendete, schillernde und sinnvariable Begriff nicht notwendig

${ }^{15}$ Vgl. S. Kott Der Sozialstaat, in: E. François/H. Schulze (Hrsg.) Deutsche Erinnerungsorte II, 2001, 485 (486f., 501); s. auch: H. Butzer Die Sozialstaatsentwicklung unter dem Grundgesetz, 2006, $50 \mathrm{ff}$; U. Steiner Der deutsche Sozialstaat auf dem Weg nach Europa - Sind christliche Sozialvorstellungen dabei?, Kirche\&Recht 2005, 133 (135, 139); U. Di Fabio Soziale Gerechtigkeit und Verfassung, Politische Studien, Heft 406 , 2006, 51 (53) spricht von einem Kennzeichen der Nationalkultur. - Zum Wunsch der deutschen Gesellschaft nach Verringerung der sozialen Unterschiede und zum Bedürfnis nach sozialer Sicherheit: R. Pitschas Frei - sozial - auch sicher?, FS Siedentopf, 2008, 285 (296f.).

16 Zur Bedeutung des sozialen Ausgleichs für den inneren Frieden: C. Starck Soziale Rechte in Verträgen, Verfassungen und Gesetzen, GS Tettinger, 2007, 761 (766ff.); ders. Der demokratische Verfassungsstaat, 1995, $235 \mathrm{ff}$. - Zu sozialen Differenzierungs-, Spannungs- und Konfliktlinien in der deutschen Gesellschaft: S. Lessenich/F. Nullmeier (Hrsg.) Deutschland - eine gespaltene Gesellschaft, 2006.

17 Zum Begriff der sozialen Gerechtigkeit und seinem Verhältnis zum Begriff der sozialen Gleichheit: J. Isensee Gerechtigkeit - zeitlose Idee im Verfassungsstaat der Gegenwart, in: H. Schmidinger (Hrsg.) Gerechtigkeit heute: Anspruch und Wirklichkeit, 2000, 253 (278ff.); s. auch: E. Eichenhofer Sozialrecht und soziale Gerechtigkeit, JZ 2005, 209 ff.; Petersen Gefühlte Ungerechtigkeit (Fn. 14), 5; G. Roellecke Gerechtigkeit und soziale Gerechtigkeit, ZRph 2004, $17 \mathrm{ff}$;; aus philosophischer und soziologischer Sicht statt Vieler mwN: S. Empter/R. B. Vehrkamp (Hrsg.) Soziale Gerechtigkeit - eine Bestandsaufnahme, 2007; S. Gosepath Gleiche Gerechtigkeit, 2004, $29 \mathrm{ff}$; ders. Soziale Gerechtigkeit - philosophische Grundlagen der Sozialstaatsbegründung, Soziale Sicherheit (CHSS) 2005, $190 \mathrm{ff}$; R. Kramer Soziale Gerechtigkeit - Inhalt und Grenzen, 1992; W. Kersting (Hrsg.) Politische Philosophie des Sozialstaats, 2000; ders. Kritik der Gleichheit, 2002; ders. Facetten der Gerechtigkeit, Leviathan 2007, $193 \mathrm{ff}$.

18 Zum politischen Gebrauch des Begriffs: H. M. Heinig Der Sozialstaat im Dienst der Freiheit, 2008, $134 \mathrm{ff}$. 
dem der sozialen Gleichheit entspricht, sondern über diesen hinausreichend auch weiteren Inhalten offen steht, ${ }^{19}$ wird er heute maßgeblich vom Gleichheitsgedanken her bestimmt ${ }^{20}$ und zielt zentral auf soziale Gleichheit.

Die Forderung nach sozialer Gleichheit richtet sich auf die Herstellung und Hebung sozialer Normalität ${ }^{21}$ und bezieht sich in erster Linie noch immer auf den Nationalstaat. Ungeachtet der Bedeutung der europäischen Integration und des Gemeinschaftsrechts für das Soziale, hingewiesen sei nur auf die Einwirkungen der Grundfreiheiten, der Diskriminierungsverbote oder auf das zukunftsoffene Potential der Unionsbürgerschaft und der sozialen Grundrechte, ${ }^{22}$ ist dieser immer noch - auch aufgrund unterschiedlicher sozialpolitischer Traditionen in den Mitgliedsstaaten - die wichtigste Ebene zur Gewährleistung von sozialer Sicherheit und sozialem Ausgleich. ${ }^{23}$ Doch ist nicht zu über-

19 Eichenhofer Sozialrecht und soziale Gerechtigkeit (Fn. 17), $210 \mathrm{ff}$.

20 Vgl. etwa: R. Herzog, in: T. Maunz/G. Dürig, Grundgesetz, Stand des Gesamtwerkes: 2008, Art. 20 VIII Rn. 36: „starke Gleichheitskomponente“; s. auch W. Leisner Der Gleichheitsstaat, 1980, 116.

${ }^{21}$ Zur sozialen Normalität als Ziel und den Schwierigkeiten, diese zu bestimmen: H. F. Zacher Das soziale Staatsziel, HStR II, 3. Aufl. 2004, § 28 Rn. 68 ff.; s. auch: R. Gröschner, in: H. Dreier (Hrsg.) Grundgesetz, Bd. I, 2. Aufl. 2004, Art. 20 (Sozialstaat) Rn. 37.

22 Vgl. dazu allgemein: $U$. Becker Schutz und Implementierung von EU-Sozialstandards, in: ders./B. Baron v. Maydell/A. Nußberger, Die Implementierung internationaler Sozialstandards, 2006, $139 \mathrm{ff}$; E. Eichenhofer Sozialrecht der Europäischen Union, 3. Aufl., 2006; ders. Geschichte des Sozialstaats in Europa, 2007, 68 ff.; W. Kahl Freiheitsprinzip und Sozialprinzip in der Europäischen Union, FS R. Schmidt, 2006, 75 ff.; T. Kingreen Das Sozialstaatsprinzip im europäischen Verfassungsverbund, 2003, 283 ff.; R. Schlegel Gesetzliche Krankenversicherung im Europäischen Kontext, SGb 2007, $700 \mathrm{ff}$; M. Wallerath Der Sozialstaat in der Krise, JZ 2004, 949 (950ff.); Zacher Staatsziel (Fn. 21), § 28 Rn. $145 \mathrm{ff}$; zu den Auswirkungen des Vertrages von Lissabon auf das "Soziale“: C. Schnell/J. Wesenberg Der Reformvertrag von Lissabon und seine sozialpolitischen Auswirkungen - eine Bestandsaufnahme, DRV 2008, $275 \mathrm{ff}$; zu den Sozialstandards der EMRK: C. Grabenwarter Sozialstandards in der Europäischen Menschenrechtskonvention, in: U. Becker/B. Baron v. Maydell/A. Nußberger, Die Implementierung internationaler Sozialstandards, 2006, $83 \mathrm{ff}$. - Aus soziologischer Sicht zur Europäisierung sozialer Ungleichheiten: M. Heidenreich (Hrsg.) Die Europäisierung sozialer Ungleichheiten, 2006.

${ }^{23}$ Zur grundgesetzlichen Sozialstaatlichkeit als nationale Sozialstaatlichkeit D. Merten Grenzen des Sozialstaates, VSSR 1995, 155 (167f.); s. auch: Heinig Sozialstaat (Fn. 18), $408 \mathrm{ff}$., der darauf hinweist, dass soziale Hilfen für Ausländer im Ausland verfassungssystematisch nicht dem Art. 20 Abs. 1 GG zugeordnet sind, sondern ihre verfassungsrechtlichen Grundlagen im Normkomplex des offenen Verfassungsstaats finden (ebd., 410). 
sehen, dass gerade auf der Grundlage universeller Sozialstandards ${ }^{24}$ und der mit der Globalisierung ${ }^{25}$ verbundenen $\mathrm{Ab}$ - und Zuwanderung von Menschen und Kapital die Forderung nach sozialer Gleichheit über die jeweiligen Staaten hinausreicht und europa- oder weltweit erhoben werden kann und wird. Unabhängig von ihrem Bezugspunkt zielt die Forderung nach sozialer Gleichheit immer auf die jeweilige soziale Mitte, verstanden als der gesellschaftliche Standard, der allerdings einem stetigen Wandel unterliegt. ${ }^{26}$ Orientiert sich die Forderung nach sozialer Gleichheit damit an einer relativen Größe, so bleibt ihre generelle Zielrichtung davon unberührt: gegen Subnormalität, auf Zugang zur und Verbleib in der Normalität sowie auf Hebung der Normalität. ${ }^{27}$

Bezog sich die soziale Frage des 19. Jahrhunderts insbesondere auf die Arbeiterschaft als benachteiligte Gruppe, so wurden in der Folgezeit immer neue Konstellationen individueller oder gruppenhafter sozialer Schlechterstellungen identifiziert, ${ }^{28}$ etwa in Bezug auf Alleinerziehende, Personen mit Migrationshintergrund, Mieter oder Verbraucher. Soziale Ungleichheiten werden wahrgenommen im Hinblick auf die individuelle Lebenssituation (krank, alt oder behindert), auf den Lebensraum (StadtLand, alte-neue Bundesländer) oder hinsichtlich einzelner Sachbereiche, etwa bei der Bildung oder in Bezug auf Freizeit- und Umweltbedingungen. Heute gelten Prekarität und Prekariat als Signalwörter neuer sozialer Ungleichheiten. ${ }^{29}$

Mit der zunehmenden Pluralisierung von Milieus und Lebensstilen scheinen die relevanten Vergleichsgruppen und Sachverhalte sozialer Ungleichheit unbegrenzt vermehrbar und nahezu unendlich zu sein. Selbst wenn fundamentale Ungleichheitsrelationen die Peripheren in

\footnotetext{
${ }^{24} \mathrm{Zu}$ den Quellen und zur Bedeutung internationaler Sozialstandards grundlegend: A. Nußberger, Sozialstandards im Völkerrecht, 2005; s. auch: U. Becker/B. Baron v. Maydell/A. Nußberger Die Implementierung internationaler Sozialstandards, 2006; E. Eichenhofer Menschenrecht auf soziale Sicherheit, VSSR 2007, $87 \mathrm{ff}$.; M. Kotzur Soziales Völkerrecht für eine solidarische Völkergemeinschaft?, JZ 2008, 265 ff.

25 Allgemein zu den Aus- und Einwirkungen der Globalisierung auf den nationalen Sozialstaat $H$. Bauer Die Verfassungsentwicklung des wiedervereinten Deutschlands, HStR I, 3. Aufl. 2003, § 14 Rn. 104.

${ }^{26}$ Gröschner, in: Dreier (Fn. 21), Art. 20 (Sozialstaat) Rn. 37.

27 Zacher Staatsziel (Fn. 21), § 28 Rn. 71.

28 S. dazu Zacher Staatsziel (Fn. 21), § 28 Rn. 35. - Analyse sozialer Ungleichheiten aus soziologischer Sicht bei S. Hradil Soziale Ungleichheit in Deutschland, 8. Aufl. 2001, $147 \mathrm{ff}$.

${ }^{29}$ B. Vogel Prekarität und Prekariat - Signalwörter neuer sozialer Ungleichheiten, APuZ 2008, Heft 33-34, 12 ff.; ders. Staatsbedürftigkeit der Gesellschaft (Fn. 11), 81 ff.
} 
den Hintergrund treten lassen, ${ }^{30}$ öffnet der Ausgleich einer anerkannten sozialen Ungleichheit den Blick auf neue Ungleichheiten, die ihrerseits nach Kompensation rufen. ${ }^{31}$ Der Horizont sozialer Gleichheit ist in stetiger Bewegung. ${ }^{32}$

\section{Abbau sozialer Ungleichheiten und Herstellung sozialer Gleichheit: der ökonomische Bezug des Sozialen}

Die staatlicherseits zum Abbau sozialer Ungleichheit und zur Herstellung sozialer Gleichheit eingesetzten und diskutierten Instrumente sind zahlreich und vielfältig: ${ }^{33}$ Steuertarife, die eine stärkere Umverteilung zwischen reich und arm bewirken sollen, ${ }^{34}$ Sozialversicherungsreformen, die die Finanzierungsgrundlagen erweitern, Leistungen neu justieren oder den Versichertenkreis ausdehnen, die Einführung von Mindestlöhnen oder Reformen im Bildungswesen in Folge von Pisastudien. Trotz der unterschiedlichen Ansätze und Wege ist allerdings nicht zu übersehen, dass zumeist ökonomische bzw. ökonomisch wirkende Mittel und Instrumente vorgeschlagen und eingesetzt werden.

Obwohl der Begriff des Sozialen weit und offen ist, ${ }^{35}$ es sich um ein „Wiesel-Wort“ par excellence handelt, ${ }^{36}$ gelten im juristischen Kontext

${ }^{30}$ Dazu: O. Depenheuer Das soziale Staatsziel und die Angleichung der Lebensverhältnisse in Ost und West, HStR IX, 1997, § 204 Rn. 63.

${ }_{31}$ Zacher Staatsziel (Fn. 21), § 28 Rn. 39; s. auch: Depenheuer Angleichung der Lebensverhältnisse (Fn. 30), § 204 Rn. 63; M. Stolleis Historische und ideengeschichtliche Entwicklung des Gleichheitssatzes, in: R. Wolfrum (Hrsg.) Gleichheit und Nichtdiskriminierung im nationalen und internationalen Menschenrechtsschutz, 2003, 7 (9f.).

32 Bezogen auf soziale Gerechtigkeit: J. Isensee Verfassung ohne soziale Grundrechte, Der Staat 19 (1980), 367 (375).

${ }^{33}$ Vgl. Zacher Staatsziel (Fn. 21), § 28 Rn. 36.

${ }^{34} \mathrm{Zu}$ den tatsächlichen Auswirkungen der Steuerprogression vgl. den dritten Armuts- und Reichtumsbericht (Fn. 2), 34f. Danach tragen die obersten $10 \%$ der Einkommensteuerpflichtigen zu $52 \%$ des gesamten Einkommensteueraufkommens bei, die untersten $50 \%$ zu gut $6 \%$. - Zum Leistungsfähigkeitsprinzip als Grundlage der Einkommensteuererhebung mit jeweils weiteren Nachweisen: D. Birk Das Leistungsfähigkeitsprinzip als Maßstab der Steuernormen, 1983; P. Kirchhof Die Steuern, HStR V, 3. Aufl. 2007, § 118 Rn. 182 ff.; L. Lammers Die Steuerprogression im System der Ertragsteuern und ihr verfassungsrechtlicher Hintergrund, 2007, 69ff.; J. Lang, in: K. Tipke/J. Lang, Steuerrecht, 19. Aufl. 2008, § 4 Rn. 81 ff.; C. Waldhoff Grundzüge des Finanzrechts des Grundgesetzes, HStR V, 3. Aufl. 2007, § 116 Rn. $100 \mathrm{ff}$.

${ }^{35}$ Zum Begriff des „Sozialen“ grundlegend: H. F. Zacher Der europäische Sozialstaat, SZS/RSAS 2008, 1 (2ff.); ders. Das „Soziale“ als Begriff des deutschen und europäischen Rechts, in: Deutsche Rentenversicherung Bund (Hrsg.) Das Soziale in der Alterssicherung, 2005, $11 \mathrm{ff}$.

${ }^{36}$ F. A. von Hayek Wissenschaft und Sozialismus, 1979, 3 (16). 
vor allem solche sozialen Ungleichheiten als problematisch, die in ökonomischen Ungleichheiten ihre Ursache oder ihre Wirkung haben. ${ }^{37}$ Schon die soziale Frage des 19. Jahrhunderts ${ }^{38}$ wies einen starken ökonomischen Bezug auf und zielte zentral auf die Verbesserung der ökonomischen Verhältnisse. ${ }^{39}$ Selbst zu Beginn des 21. Jahrhunderts bezieht sich staatliche Sozialgestaltung im Wesentlichen noch immer auf die ökonomische Basis der Gesellschaft, ${ }^{40}$ wiewohl unbestritten ist, dass sich das Soziale nicht auf das Ökonomische reduzieren lässt und nichtökonomische Interventionen für das Soziale ebenso relevant wie notwendig sind, ${ }^{41}$ denn soziale Bedürftigkeit ist nicht immer als ökonomische Insuffizienz bezifferbar und einem ökonomischen Ausgleich zugänglich. ${ }^{42}$ Finanzielle Hilfen können im Gegenteil sogar zu dauerhafter Abhängigkeit und Verfestigung von Armut führen, so dass es ebenfalls wirksamer, allerdings wiederum kostenintensiver Aktivierungsprogramme bedarf, um vom Bezug von Transferleistungen unabhängig zu werden.

In einer marktwirtschaftlich organisierten arbeitsteiligen Gesellschaft eröffnet ein ausreichendes Einkommen den Zugang zu den meisten Gütern der Gesellschaft; es schafft Sicherheit und erweitert Handlungsspielräume. Solange Erwerbsarbeit für die meisten Menschen den bei weitem wichtigsten Zugang zur Erzielung von Einkommen und zur Teilhabe am gesellschaftlichen Leben bedeutet, ${ }^{43}$ zielt die Forderung nach sozialer Gleichheit auch auf den erleichterten Zugang zu Arbeit, insbesondere über den Ausbau von Bildungs- und Weiterbildungsmöglich-

37 Zacher Staatsziel (Fn. 21), § 28 Rn. 35.

38 Zur sozialen Frage und zur Entwicklung der Sozialpolitik und des Sozialrechts im 19. Jahrhundert: T. Nipperdey Deutsche Geschichte 1866-1918, Bd. I, 1990, 335 ff.; M. Stolleis Geschichte des Sozialrechts in Deutschland, 2003, $23 \mathrm{ff}$.; s. auch: R. Breuer Grundlagen des Sozialstaates, GS Heinze, 2005, 81 (83ff.).

${ }^{39}$ Dies gilt insbesondere im Hinblick auf die Arbeitsbedingungen sowie die Absicherung des Einkommensausfalls durch Krankengeld oder Rente. Allgemein dazu: Zacher Staatsziel (Fn. 21), § 28 Rn. $34 \mathrm{f}$.

${ }^{40}$ K.-J. Bieback Sozialstaatsprinzip und Grundrechte, EuGRZ 1985, 657 (659).

41 Vgl. dazu Zacher Staatsziel (Fn. 21), § 28 Rn. $72 \mathrm{f}$.

42 H. F. Zacher Soziale Gleichheit, AöR 93 (1968), 341 (343).

43 Zur Bedeutung der Arbeit und den Veränderungen des Arbeitsmarktes: M. Wallerath Arbeitsmarkt, HStR IV, 3. Aufl. 2006, § 94 Rn. 1 ff.; J. Wieland Arbeitsmarkt und staatliche Lenkung, VVDStRL 59 (2000), 13 (16ff.); C. Engel Arbeitsmarkt und staatliche Lenkung, ebd., 56 (58ff.); aus rechtsvergleichender und europarechtlicher Sicht: T. von Danwitz Arbeitsmarkt und staatliche Lenkung, ebd., 99 (100ff.). - Statistisches Material zur Entwicklung auf dem Arbeitsmarkt im dritten Armuts- und Reichtumsbericht (Fn. 2), $65 \mathrm{ff}$. 
keiten. ${ }^{44}$ Doch verursacht die Vermittlung von mehr Bildung grundsätzlich auch mehr Kosten, was zugleich wiederum den ökonomischen Bezug sozialer Gleichheit verdeutlicht. Weil Arbeit, Einkommen und Vermögen noch immer wesentlich über die Handlungsoptionen des Einzelnen in der Gesellschaft entscheiden, richtet sich die Forderung nach sozialer Gleichheit im Kern gegen unangemessene Ungleichheit in den wirtschaftlichen Lebensbedingungen und verlangt deren Angleichung und Anhebung.

\section{Soziale Gleichheit als Aufgabe von Staat und Gesellschaft}

In der freiheitlichen demokratischen Wirtschafts- und Sozialordnung des Grundgesetzes sind die Angleichung und Verbesserung der Lebensverhältnisse weder alleinige noch primäre Aufgaben des Staates. Das grundgesetzliche Sozialstaatsprinzip normiert zwar ein Staatsziel, jedoch keinen Staatsvorbehalt für das Soziale. ${ }^{45}$ Im Gegenteil: Sozialstaatliche Aktivität reibt sich an den grundrechtlichen Vorbehalten zugunsten privater Tätigkeit zur Steigerung und Angleichung der materiellen Lebensbedingungen. In den Grundrechten kommt gerade der Vorrang der gesellschaftlichen Realisation, etwa in der Familie, durch Kirchen oder durch sonstiges bürgerschaftliches Engagement ${ }^{46}$, gegenüber staatlicher Intervention zur Herstellung sozialer Gleichheit zum Ausdruck. ${ }^{47}$

Staatliche Aktivitäten können vor dem Hintergrund des Subsidiaritätsprinzips ${ }^{48}$ erst dann Raum greifen, wenn vorrangige private Eigenvorsorge ${ }^{49}$ und die grundrechtlich geschützte gesellschaftliche Selbstregulierung den Erfordernissen sozialer Gleichheit nicht mehr genügt und die Resultate des Marktprozesses der Korrektur bedürfen. Der Abbau sozialer Ungleichheit ist zwar eine Staatsaufgabe, doch ist diese dem Staat nur im Sinne einer Letzt- und Gesamtverantwortung zugewiesen.

\footnotetext{
${ }^{44}$ Vgl. dazu den dritten Armuts- und Reichtumsbericht (Fn. 2), $58 \mathrm{ff}$.

45 J. Isensee Staatsaufgaben, HStR IV, 3. Aufl. 2006, § 73 Rn. 68.

46 Dazu R. Pitschas Soziale Sicherungssysteme im „europäisierten“ Sozialstaat, FS 50 Jahre Bundesverfassungsgericht, Bd. II, 2001, 827 (844).

47 A. Nußberger Soziale Gleichheit - Voraussetzung oder Aufgabe des Staates, DVB1. 2008, 1081 (1087f.). - Zur Frage, inwieweit steuerrechtliche Anreize durch das Gemeinnützigkeitsrecht für gemeinwohlorientiertes Engagement Privater möglich und zulässig sind: $M$. Heintzen Steuerliche Anreize für gemeinwohlorientiertes Engagement Privater, FR 2008, $737 \mathrm{ff}$.

${ }^{48}$ Zum Subsidiaritätsprinzip grundlegend: J. Isensee Subsidiaritätsprinzip und Verfassungsrecht, 2. Aufl. 2001.

${ }^{49}$ Zur sozialen Eigenvorsorge im subsidiären Sozialstaat Pitschas Sicherungssysteme (Fn. 46), $828 \mathrm{ff}$.
} 
Die wirtschaftlichen Lebensbedingungen unterliegen in einer europäisierten Marktwirtschaft nicht umfassender staatlicher Befehls-, Planungs- und Verfügungsmacht, sondern entspringen grundrechtlicher Freiheit. ${ }^{50}$ Durch Recht allein lässt sich Wohlstand nicht verordnen und erzielen. Die ökonomischen Mittel, die der Staat einsetzt und einsetzen muss, um soziale Gleichheit über Umverteilung herzustellen, müssen als solche erst in und von der Gesellschaft erwirtschaftet werden, denn die zur Verteilung notwendigen Mittel schöpft der Staat aus der Erhebung von Steuern und sonstigen Abgaben, damit aus der Teilhabe am wirtschaftlichen Erfolg seiner Bürger. ${ }^{51}$ Wirtschaftliche Produktivität und Prosperität sind somit Voraussetzungen, um soziale Gleichheit durch staatliche Maßnahmen herzustellen. ${ }^{52}$ Der Staat kann und muss daher, um soziale Gleichheit zu erreichen, auch Rahmenbedingungen zur Ermöglichung wirtschaftlicher Prosperität - wozu auch Kontrollen und Regulierungen zählen - schaffen, die er selbst als solche nicht unmittelbar und exklusiv herstellen kann. ${ }^{53}$

\section{Sinn und Zweck sozialer Gleichheit}

\section{Soziale Gleichheit als eine Voraussetzung staatlicher Einheit}

Übermäßige soziale Spannungen bergen die Gefahr in sich, dass Demokratien zerbrechen. ${ }^{54}$ "Ein bestimmtes Maß sozialer Homogenität“ so Hermann Heller $1928^{55}$ - „muss gegeben sein, damit politische Einheitsbildung überhaupt möglich sein soll“. Herbert Krüger nennt es eine

50 J. Isensee Der Sozialstaat in der Wirtschaftskrise, FS Broermann, 1982, 365 (368).

51 H.-J. Papier Zur Zukunft des Sozialstaates, ZFSH/SGB 2006, 3 (4); s. dazu auch: E. Benda Der soziale Rechtsstaat, in: ders./W. Maihofer/H.-J. Vogel (Hrsg.) Handbuch des Verfassungsrechts, 2. Aufl. 1995, § $17 \mathrm{Rn}$. $175 \mathrm{ff}$.

52 Zum Verhältnis von Sozialstaat und Prosperität grundlegend: H. F. Zacher Sozialstaat und Prosperität, FS R. Schmidt, 2006, $305 \mathrm{ff}$.

53 Vgl. O. Depenheuer Setzt Demokratie Wohlstand voraus?, Der Staat 33 (1994), 329 (346).

54 W. Rüfner, in: Bonner Kommentar, Grundgesetz, Stand des Gesamtwerkes: 2008, Art. 3 Abs. 1 Rn. 55. - B.-O. Bryde Steuerverweigerung und Sozialstaat, FS v. Zezschwitz, 2005, 321 (332), weist daraufhin, dass die Verhinderung zu exzessiver Ungleichheit der Vermögensverteilung im Interesse funktionierender Demokratie liege, die erfahrungsgemäß in Ländern mit extremen Vermögensdisparitäten Schwierigkeiten habe.

55 Politische Demokratie und soziale Homogenität, in: ders., Gesammelte Schriften, Bd. II, 1971, 421 (427). - Zu Person und Werk von H. Heller: G. Robbers H. Heller: Staat und Kultur, 1983. 
„alte These“, dass politische Demokratie nicht möglich sei, „wenn allzu große Vermögensunterschiede vorwalteten" und spricht insoweit von einer negativen Verfassungsvoraussetzung. ${ }^{56} \mathrm{Um}$ seiner selbst willen muss der demokratische Verfassungsstaat des Grundgesetzes zum Erhalt seiner Stabilität auf ein Mindestmaß an sozialer Gleichheit achten, diese wahren und verhindern, dass die sozialen Unterschiede unangemessen groß werden. ${ }^{57}$ Ausdrücklich normiert auch der Staatenverbund „Europäische Union“ in den Verträgen die Stärkung des wirtschaftlichen und sozialen Zusammenhalts als ein Ziel, 58 „um eine harmonische Entwicklung "59 $\mathrm{zu}$ fördern und den dauerhaften Erfolg der Union zu sichern. Ein Mindeststandard sozialer Homogenität ${ }^{60}$ wird damit auch für die Europäische Union als eine Funktionsbedingung angesehen. ${ }^{61}$

Selbst wenn im sozialen Bundesstaat ${ }^{62}$ des Grundgesetzes die unitarisierend wirkende Forderung nach sozialer Gleichheit föderal gebrochen ist, weil der Bundesstaat Vielfalt und Unterschiede vorsieht und fordert, damit auch regionale soziale Ungleichheiten zulässt, bedarf es „gleichwertiger Lebensverhältnisse" 63 im Bundesgebiet, um erhebliche Wande-

56 Verfassungsvoraussetzungen und Verfassungserwartungen, FS Scheuner, 1973, 285 (287).

57 Zum sozialen Ausgleich als Staatsziel: C. Starck Verfassungsstaat (Fn. 16), 237.

58 Art. 2 Abs. 1, 1. Spstr. EU, Art. 2 EG, Art. 3 lit. k EG, Art. 158ff. EG; s. auch im Vertrag von Lissabon: Art. 3 Abs. 3, UA 2, 3; Art. 209; Art. 220; zur Kohäsion als Politikziel der Union und Gemeinschaft: P. Gussone Das Solidaritätsprinzip in der Europäischen Union und seine Grenzen, 2006, $87 \mathrm{ff}$.; A. Puttler, in: C. Calliess/M. Ruffert (Hrsg.) EUV/EGV, 3. Aufl. 2007, Art. 158 EG Rn. 1 ff.

59 Art. 158 Abs. 1 EG.

${ }^{60}$ Zum Begriff der Homogenität allgemein: O. Depenheuer Solidarität im Verfassungsstaat, Typoskript, 1992, $268 \mathrm{ff}$; H. Dreier, in: ders. (Hrsg.) Grundgesetz, Bd. II, 2. Aufl. 2006, Art. 28 Rn. 3; F. Schorkopf Homogenität in der europäischen Union Ausgestaltung und Gewährleistung durch Art. 6 Abs. 1 und Art. 7 EUV, 2000, $28 \mathrm{ff}$.

${ }^{6} 1$ Vgl. J. Isensee Soziale Sicherheit im europäischen Markt, VSSR 1996, 169 (179f.); s. auch: ders. Nachwort, in: ders. (Hrsg.) Europa als politische Idee und als rechtliche Form, 2. Aufl. 1994, 103 (122 ff.).

62 Zur Problematik des sozialen Bundesstaates zum Spannungsfeld zwischen Bundesstaatsprinzip und Sozialstaatsprinzip: S. Boysen Gleichheit im Bundesstaat, 2005; Depenheuer Angleichung der Lebensverhältnisse (Fn. 30), § 204 Rn. 109ff.; N. Engels Chancengleichheit und Bundesstaatsprinzip, 2001; Zacher Staatsziel (Fn. 21), § 28 Rn. $97 \mathrm{ff}$.

${ }^{63} \mathrm{Zu}$ Inhalt und Bedeutung des Begriffs „gleichwertiger Lebensverhältnisse“ in Art. 72 Abs. 2 GG, der die ursprüngliche Formulierung „Einheitlichkeit der Lebensverhältnisse“ ersetzte: BVerfGE 106, 62 (142ff.); 112, 226 (253); S. Oeter, in: H. v. Mangoldt/F. Klein/C. Starck (Hrsg.) Grundgesetz, Bd. II, 5. Aufl. 2005, Art. 72 Rn. 90 ff.; R. Sannwald, in: B. Schmidt-Bleibtreu/H. Hofmann/A. Hopfauf (Hrsg.) Grundgesetz, 11. Aufl. 2008, Art. 72 Rn. 49 ff. 
rungsbewegungen von Menschen, Arbeit oder Kapital zu verhindern, die das „bundesstaatliche Sozialgefüge" 64 beeinträchtigen und die Einheit des Bundesstaates gefährden könnten. ${ }^{65}$ Zwar lassen sich weder der Kompetenzbestimmung des Art. 72 Abs. 2 GG noch dem Bundesstaatsprinzip konkrete verfassungsunmittelbare Ansprüche entnehmen, ${ }^{66}$ doch ist ein Mindestmaß an Gleichwertigkeit in den Lebensverhältnissen, ${ }^{67}$ damit auch an sozialer Gleichheit, eine sozialstaatliche Verfassungsvoraussetzung. ${ }^{68}$ Wie wirkmächtig gerade die Forderung nach sozialer Gleichheit gegenüber dem Verlangen nach föderativer Vielfalt geworden ist, wie stark die zentripetalen Kräfte des Sozialen wirken und zu einem „Abstieg des Föderalismus im Sozialstaat“69 geführt haben,

64 BVerfGE 106, 62 (144); 111, 226 (253).

65 Depenheuer Angleichung der Lebensverhältnisse (Fn. 30), § 204 Rn. 112.

66 Zur Diskussion, ob aus Art. 72 Abs. 2 GG oder dem Bundesstaats- bzw. Sozialstaatsprinzip eine Rechtsverpflichtung des Bundes im Sinne eines Verfassungsgebots folgt mwN: H.-W. Arndt Zur verfassungsrechtlichen Problematik der Herstellung einheitlicher Lebensverhältnisse in der Bundesrepublik Deutschland, JuS 1993, $360 \mathrm{ff}$; H. Hohmann Der Verfassungsgrundsatz der Herstellung einheitlicher Lebensverhältnisse im Bundesgebiet, DÖV 1991, 191 (192ff.); S. Oeter Integration und Subsidiarität im deutschen Bundesstaatsrecht, 1998, 532 ff.; ders., in: v. Mangoldt/Klein/Starck (Fn. 63), Art. 72 Rn. 97; zu der Diskussion insbesondere aus dem Blickwinkel der Regelungen in der Finanzverfassung: W. Heun, in: H. Dreier (Hrsg.) Grundgesetz, Bd. III, 2. Aufl. 2008, Art. 107 Rn. 10; P. Selmer Grundsätze der Finanzverfassung des vereinten Deutschlands, VVDStRL 52 (1953), 10 (19ff.); F. Kirchhof Grundsätze der Finanzverfassung des vereinten Deutschlands, ebd., 69 (83f.); S. Korioth Die Finanzausstattung der neuen Bundesländer, DVB1. 1991, 1048 (1054ff.); R. Wendt Neuorientierung der Aufgaben- und Lastenverteilung im „sozialen Bundesstaat“, Staatswissenschaft und Staatspraxis 1993, 56 (59f.).

${ }^{67}$ Das Mindestmaß ist keine statische, abstrakt feststehende Größe, sondern hängt in seinem Umfang von der jeweiligen Wirtschaftslage und den jeweiligen sozialen Verhältnissen ab; vgl. Kirchhof Grundsätze der Finanzverfassung (Fn. 66), 84.

${ }^{68} \mathrm{Vgl}$. C. Waldhoff Verfassungsrechtliche Vorgaben für die Steuergesetzgebung im Vergleich Deutschland - Schweiz, 1997, $89 \mathrm{ff}$; ders. Finanzautonomie und Finanzverflechtung in gestuften Rechtsordnungen, VVDStRL 66 (2006), 216 (248ff.); s. auch Depenheuer Angleichung der Lebensverhältnisse (Fn. 30), § 204 Rn. 45 f.; Arndt Herstellung einheitlicher Lebensverhältnisse (Fn. 66), 362, spricht von einem restriktiv auszulegenden Verfassungsauftrag; ablehnend mit grundsätzlicher Kritik an der Lehre von den Verfassungsvoraussetzungen: C. Möllers Staat als Argument, 2000, $257 \mathrm{ff}$. $\mathrm{Zu}$ Bedeutung, Inhalt und Funktion der Verfassungsvoraussetzungen grundlegend: $J$. Isensee Grundrechtsvoraussetzungen und Verfassungserwartungen an die Grundrechtsausübung, HStR V, 2. Aufl. 2000, § 115 Rn. 1ff.; Krüger Verfassungsvoraussetzungen (Fn. 56), $285 \mathrm{ff}$.

69 W. Schmitt Glaeser Rechtspolitik unter dem Grundgesetz, AöR 107 (1982), 337 (355). 
zeigt sich gerade in der Realität des deutschen Bundesstaats, der durch sozialstaatlichen Unitarismus in Vollzug und Leistungen geprägt ist. ${ }^{70}$

In viel höherem Grade als jede andere politische Form ist die Demokratie - so Hermann Heller - in ihrer „Existenz von dem Dasein einer sozialen Angeglichenheit abhängig “71. In einer freiheitlichen Demokratie wächst die staatliche Einheit vor allem aus der Integrationsbereitschaft und -fähigkeit der Bürger, ${ }^{72}$ die ihrerseits auch darauf beruht, dass der soziale Status des Einzelnen bereits eine gewisse Angleichung und Verdichtung erfahren hat ${ }^{73}$ und ein „Wir-Bewusstsein und -Gefühl“"74 entstehen lässt. ${ }^{75}$ Dies verlangt nicht die Schaffung strikt gleicher, gesellschaftliche Pluralität und Verschiedenheit einebnender Lebensbedingungen. Vielmehr erforderlich ist der Ausgleich grober, als nicht hinnehmbar geltender sozialer Ungleichheiten, welche die Dazugehörigkeit zur jeweiligen Rechts-, Wirtschafts- und Kulturgemeinschaft gefährden

${ }^{70}$ Dazu J. Isensee Idee und Gestalt des Föderalismus im Grundgesetz, HStR IV, 2. Aufl. 1999, § 98 Rn. 249 ff.; ders. Der Föderalismus und der Verfassungsstaat der Gegenwart, AöR 115 (1990), 248 (255f.); R. Scholz Zum Verfassungsprinzip des „sozialen Bundesstaates“, FS Mußgnug, 2005, 19 ff.; Zacher Staatsziel (Fn. 21), § 28 Rn. 98; ders. Der soziale Bundesstaat, FS Schmitt Glaeser, 2003, 199 ff.; s. auch H. Hofmann Die Entwicklung des Grundgesetzes von 1949 bis 1990, HStR I, 3. Aufl. 2003, § 9 Rn. 72: „Egalisierung verlangt Zentralisierung“; V. Mehde Wettbewerb zwischen Staaten, 2005, $147 \mathrm{ff}$.

71 Heller Politische Demokratie und soziale Homogenität (Fn. 55), 429.

72 Depenheuer Angleichung der Lebensverhältnisse (Fn. 30), § 204 Rn. 129. - Zur Bedeutung der inneren Integration: A. Uhle Innere Integration, HStR IV, 3. Aufl. 2006, $\S 82 \mathrm{Rn} .1 \mathrm{ff}$.

${ }^{73}$ Zur Bedeutung des „Sozialen“ für den Zusammenhalt der Gesellschaft: Zacher Sozialstaat und Prosperität (Fn. 52), 327 f.; s. auch: U. Steiner Sozialer Konflikt und sozialer Ausgleich - Zur Rolle der Verfassungsgerichtsbarkeit, FS Schmitt Glaeser, 2003, 335 (340).

${ }^{74}$ Heller Politische Demokratie und soziale Homogenität (Fn. 55), 428: „Soziale Homogenität ist immer ein sozial-psychologischer Zustand, in welchem die stets vorhandenen Gegensätzlichkeiten und Interessenkämpfe gebunden erscheinen durch ein WirBewußtsein und -Gefühl, durch einen sich aktualisierenden Gemeinschaftswillen.“.

${ }^{7}$ R. H. Herzog Demokratie und Gleichheit heute, DVB1 1970, 713 (715), spricht von „einem Minimum an sozialer Gerechtigkeit“. - Zur Bedeutung eines Mindestmaßes an sozialem Ausgleich für die Demokratie: Depenheuer Setzt Demokratie Wohlstand voraus? (Fn. 53), 332 ff.; s. auch: H. Hofmann Vielfalt, Sicherheit und Solidarität statt Freiheit, Gleichheit, Brüderlichkeit?, in: J. Bizer/H.-J. Koch (Hrsg.), Sicherheit, Vielfalt, Solidarität, 1998, 101 (110); offener dagegen: U. K. Preuß Solidarität unter den Bedingungen von Vielfalt, Anmerkungen zu einem neuen Paradigma, ebd., $125 \mathrm{ff}$.; zum Einfluss ethischer Homogenität auf die Sozialstaatlichkeit: E. Wiederin Sozialstaatlichkeit im Spannungsfeld von Eigenverantwortung und Fürsorge, VVDStRL 64 (2005), 53 (81f.); zu den Voraussetzungen der Demokratie als Staats- und Regierungsform allgemein: E.-W. Böckenförde Demokratie als Verfassungsprinzip, HStR II, 3. Aufl. 2004, § 24 Rn. $58 \mathrm{ff}$. 
können. ${ }^{76}$ Welche Ungleichheiten dies sind, lässt sich nicht abstrakt für alle Zeit bestimmen, sondern hängt ab von der Bewertung durch die jeweilige Gesellschaft, die darüber entscheidet, was als sozial ungleich gilt und ob sie sozialen Ungleichheiten ablehnend, gleichgültig oder sogar wohlwollend gegenübersteht. Bei der Beurteilung können zwischen objektiver Datenlage und subjektiver Wahrnehmung durchaus erhebliche Differenzen existieren, weil die gefühlte soziale Ungleichheit von der statistisch belegten abweichen kann. ${ }^{77}$ So sank zwar 2006 das Armutsrisiko und verbesserte sich die konjunkturelle Lage, insbesondere reduzierte sich die Arbeitslosenzahl, doch führte dies zu keinen nennenswerten Veränderungen im gesellschaftlichen Bewusstsein, im Gegenteil: die soziale Sensibilität stieg weiter an und die Zustimmung zur sozialen Marktwirtschaft sank. ${ }^{78}$ Die amerikanische - und inzwischen weltweite Bankenkrise wird insoweit - auch jenseits ihrer weit reichenden tatsächlichen Folgen - weitere Zweifel an der Marktwirtschaft nähren und die Stimmung weiter verschlechtern. Auf Empfindungen und Einstellungen kann der freiheitliche Staat jedoch allenfalls beschränkt und mittelbar einwirken, denn deren Änderung ist kein Gegenstand unmittelbarer verfassungsrechtlicher Direktiven. ${ }^{79}$

\section{Soziale Gleichheit als eine Voraussetzung der Grundrechtsausübung}

Gleiche rechtliche Freiheit führt angesichts der Verschiedenartigkeit der Menschen notwendig zu unterschiedlichen Ergebnissen, ${ }^{80}$ die sich aufgrund des rechtlichen Schutzes des Erworbenen durch Eigentumsoder Erbrechtsgarantie noch potenzieren und verfestigen können. ${ }^{81}$ Gleiche rechtliche Freiheit erzeugt soziale Ungleichheit und ist deren permanente Quelle, denn die liberalen Freiheitsrechte des Grundgesetzes bieten jedermann die gleiche rechtliche Freiheit an ohne Ansehen

\footnotetext{
${ }^{76} \mathrm{Zu}$ der Funktion des Sozialstaates, die Dazugehörigkeit zu sichern: P. Kirchhof Der allgemeine Gleichheitssatz, HStR V, 2. Aufl. 2000, § 124 Rn. 151.

77 Dazu Petersen Gefühlte Ungerechtigkeit (Fn. 14), 5.

78 Zur sinkenden Zustimmung zur sozialen Marktwirtschaft: Bertelsmann Stiftung u.a. Bürgerprogramm soziale Marktwirtschaft, 2008, $8 \mathrm{ff}$.

79 Depenheuer Angleichung der Lebensverhältnisse (Fn. 30), § 204 Rn. 41; s. dazu auch Nußberger Soziale Gleichheit (Fn. 47), 1082.

${ }^{80}$ E.-W. Böckenförde Freiheitssicherung gegenüber gesellschaftlicher Macht, in: ders., Staat, Gesellschaft, Freiheit, 1976, 336 (338); ders. Die sozialen Grundrechte im Verfassungsgefüge, in: ders/J. Jekewitz/T. Ramm (Hrsg.) Soziale Grundrechte, 1981, 7 (8f.); s. auch D. Murswiek Grundrechte als Teilhaberechte, soziale Grundrechte, HStR V, 2. Aufl. 2000, § 112 Rn. 30.

${ }^{81}$ Böckenförde, Freiheitssicherung gegenüber gesellschaftlicher Macht (Fn. 80), 338; s. auch: K. Hesse Der Gleichheitssatz im Staatsrecht, AöR 77 (1951/1952), 167 (179ff.).
} 
seiner Person, seiner Bedürfnisse, seiner Fähigkeiten oder Verdienste. ${ }^{82}$ Aus der grundrechtlichen Garantie gleicher rechtlicher Freiheit folgt die Freiheit, anders sein zu dürfen als andere; ${ }^{83}$ zur Freiheit gehört Ungleichheit. ${ }^{84}$

Die Freiheit - so bereits Lorenz von Stein - ist jedoch erst „eine wirkliche in dem, der die Bedingungen derselben, die materiellen und geistigen Güter als die Voraussetzung der Selbstbestimmung besitzt" ${ }^{\text {"85. }}$. Fehlt es an den jeweils erforderlichen Freiheitsvoraussetzungen, bleibt die Garantie der Menschenwürde unerfüllt und laufen die Freiheitsrechte leer. ${ }^{86}$ Das „Freiheitsrecht wäre ohne die tatsächliche Voraussetzung, es in Anspruch nehmen zu können, wertlos“, hieß es bereits in der - insgesamt zwar in unterschiedliche Richtungen weisenden ${ }^{87}$ - numerus-clausus Entscheidung. ${ }^{88}$ Selbst wenn Freiheit schon ein Wert an sich ist, ${ }^{89}$ bedarf es, wenn vorrangige gesellschaftliche Realisation misslingt, staatlicher Maßnahmen, damit sich Freiheit auch tatsächlich ausüben lässt. Um gleiche Freiheit für alle zur Entstehung zu bringen und um die Zugehörigkeit des Einzelnen zu einer Rechtsgemeinschaft zu gewährleisten, in der alle Menschen in ihrer Würde gleich sind und sie deshalb auch am rechtlichen, ökonomischen und kulturellen Standard dieser Gemeinschaft in gleicher Weise teilhaben sollen können, ${ }^{90}$ hat der Staat die Aufgabe, die realen Voraussetzungen der Grundrechtsausübung zu sichern und den Freiheitsrechten damit gesellschaftliche Effektivität und Breitenwirkung zu geben. ${ }^{91}$ Wären die sozialen Defizite in

82 Depenheuer Angleichung der Lebensverhältnisse (Fn. 30), § 204 Rn. 133 ff.

${ }^{83}$ P. Kirchhof Der demokratische Rechtsstaat - die Staatsform der Zugehörigen, HStR IX, 1997, § 221 Rn. 139.

${ }^{84}$ Vgl. Bundespräsident H. Köhler Zur Freiheit gehört Ungleichheit, F.A.Z. vom 29. 12. 2007, 3.

85 L. von Stein Geschichte der sozialen Bewegung in Frankreich von 1789 bis auf unsere Tage (1850), Bd. 3, Nachdruck der von G. Salomon hrsg. Ausgabe von 1921, 1972, 104.

86 Vgl. Kirchhof Armut und Freiheit (Fn. 5), 329; ders. Der demokratische Rechtsstaat (Fn. 83), § 221 Rn. 147; s. auch: ders. Grundrechtsinhalte und Grundrechtsvoraussetzungen, in: D. Merten/H.-J. Papier (Hrsg.) Handbuch der Grundrechte, Bd. I, 2004, $\S 21 \mathrm{Rn} .7 \mathrm{ff}$.

${ }^{87}$ Dazu Isensee Verfassung ohne soziale Grundrechte (Fn. 32), 372.

88 BVerfGE 33, 303 (331).

${ }^{89}$ Dazu und zur Kritik an der numerus-clausus Entscheidung: Depenheuer Setzt Demokratie Wohlstand voraus? (Fn. 53), $341 \mathrm{ff}$.

90 Kirchhof Der demokratische Rechtsstaat (Fn. 83), § 221 Rn. 139.

91 Vgl. E.-W. Böckenförde Grundrechtstheorie und Grundrechtsinterpretation, NJW 1974, 1529 (1538). 
einer Gesellschaft so groß, dass praktisch niemand mehr ein bestimmtes Grundrecht ausüben könnte, entfiele auch seine effektive Geltung. ${ }^{92}$

Soziale Gleichheit ist somit das Korrektiv zur Realität der freien Gesellschaft mit ihrer Vielfalt von Daseinsumständen und ihrer tatsächlichen Ungleichheit. Grundrechtlich geforderte soziale Gleichheit zielt auf die Gewährleistung und Sicherung der Voraussetzungen für ein selbstbestimmtes Leben des Einzelnen in der Gesellschaft. Die Grundrechte fordern damit keine selbstzweckhafte Mindestegalisierung, sondern die Gewährleistung eines freiheitsfunktional zu bemessenden sozialen Minimums an effektiven und gleichen Grundchancen zur freien Entfaltung der Persönlichkeit, um in Selbstbestimmung Grundrechte ausüben zu können. ${ }^{93}$ Soziale Gleichheit ist insoweit „eine Form der Freiheit" ${ }^{* 94}$.

\section{Inhalt und Umfang sozialer Gleichheit}

Das Grundgesetz selbst enthält, auch mangels eines umfassenden Katalogs sozialer Grundrechte, ${ }^{95}$ die auf soziale Gleichheit drängen können, keine ausdrückliche Verpflichtung zur Herstellung sozialer Gleichheit. Allerdings liegt das Ziel sozialer Gleichheit durchaus in seinem normativen Blickfeld, wenn es den Staat als sozialen Rechtsstaat konzipiert, Gleichbehandlung vorschreibt, die Herstellung gleichwertiger Lebensverhältnisse als Kompetenzausübungsvoraussetzung normiert ${ }^{96}$

92 Isensee Grundrechtsvoraussetzungen (Fn. 68), § 115 Rn. 18.

93 Grundlegend $\mathrm{zu}$ einem freiheitsfunktionalen Verständnis des Sozialstaats Heinig Sozialstaat (Fn. 18).

94 P. Häberle Grundrechte im Leistungsstaat, VVDStRL 30 (1972), 44 (96f.).

95 Zur Diskussion um das Für und Wider sozialer Grundrechte: E.-W. Böckenförde/ J. Jekewitz/T. Ramm (Hrsg.) Soziale Grundrechte, 1981; J. Lücke Soziale Grundrechte als Staatszielbestimmungen und Gesetzgebungsaufträge, AöR 107 (1982), 15 ff.; G. Lübbe-Wolff Justiziabilität sozialer Grundrechte und Verfassungsaufträge, JöR 53 (2005), 1 ff.; Murswiek Grundrechte als Teilhaberechte (Fn. 80), § 112 Rn. 40 ff.; W. Rüfner Leistungsrechte, in: Handbuch der Grundrechte, Bd. II (Fn. 5), § 40 Rn. 9 ff.; F. E. Schnapp Soziale Grundrechte aus verfassungsrechtlicher Sicht, in: B. Baron v. Maydell (Hrsg.) Soziale Rechte in der EG, 1990, 5 ff.; aus schweizerischer Sicht grundlegend: J. P. Müller Soziale Grundrechte in der Verfassung?, 2. Aufl. 1981. - Speziell zu den sozialen Grundrechten in den deutschen Landesverfassungen: A. Brenne Soziale Grundrechte in den Landesverfassungen, 2003; Zacher Staatsziel (Fn. 21), § 28 Rn. 12f., 19; zu den sozialen Grundrechten in der Europäischen Grundrechtecharta: T. Winner Die europäische Grundrechtecharta und ihre soziale Dimension, 2004.

96 Art. 72 Abs. 2 GG; s. dazu bereits oben bei Fn. 63 ff. 
oder in der Finanzverfassung einen Ausgleich zwischen den Ländern vorsieht ${ }^{97}$.

\section{Soziale Gleichheit und Rechtsstaatsprinzip}

Soziale Gleichheit bedarf des Rechtsstaats. Der Rechtsstaat sichert die ökonomischen Voraussetzungen individueller Freiheit durch die allgemeine und gleiche Gewähr der Grundrechte für Jedermann unabhängig von Stand, Vermögen und Einkommen.98 Das Rechtsstaatsprinzip leitet und begrenzt nicht nur staatliche Maßnahmen zur Herstellung sozialer Gleichheit, dadurch dass diese im Verfahren und in den Formen des Rechtsstaats erfolgen müssen, sondern es bildet vielmehr auch den Grund, auf dem sich soziale Gleichheit erst entfalten kann und steht schon deshalb nicht dem sozialen Staatsziel unvereinbar gegenüber. ${ }^{99}$ Rechtsstaat und Sozialstaat sind kompatibel und aufeinander angewiesen. Der Rechtsstaat ist notwendige Voraussetzung und Bedingung sozialer Gleichheit: ohne rechtliche Gleichheit keine soziale Gleichheit.

${ }_{97}$ Art. 104b Abs. 1 Nr. 2 GG (Ausgleich unterschiedlicher Wirtschaftskraft); Art. 107 Abs. 2 GG (Finanzausgleich); s. auch: Art. 106 Abs. 3 S. 4 Nr. 2 GG (Verteilung der Umsatzsteuer unter Wahrung der „Einheitlichkeit der Lebensverhältnisse im Bundesgebiet"). - Zu diesen finanzverfassungsrechtlichen Vorschriften: Arndt Herstellung einheitlicher Lebensverhältnisse (Fn. 66), $362 \mathrm{ff}$; K. Vogel/C. Waldhoff, in: Bonner Kommentar (Fn. 54), Vorbem. z. Art. 104a-115 Rn. 81 ff.

${ }_{98}$ Kirchhof Armut und Freiheit (Fn. 5), 325.

${ }^{99}$ Das Verhältnis von Rechtsstaat und Sozialstaat gehört spätestens seit dem Referat von E. Forsthoff „Begriff und Wesen des sozialen Rechtsstaates“ auf der Staatsrechtslehrertagung 1954 (VVDStRL 12 (1954), 8 ff.) zu den Dauerthemen juristischer Befassung mit dem Sozialstaatsprinzip und hat zu grundsätzlichen Kontroversen geführt. Inzwischen setzte sich immer stärker die Auffassung durch, dass zwischen beiden verfassungsrechtlichen Prinzipien kein unversöhnliches Gegenüber besteht, sondern beide Prinzipien gleichen Rang besitzen, sie miteinander verbunden und verschwistert sind. Vgl. dazu und zur Rezeption der "Inkommensurabilitätsthese“ von Forsthoff: Heinig Sozialstaat (Fn. 18), 22 ff.; Herzog, in: Maunz/Dürig (Fn. 20), Art. 20 VIII Rn. 30 ff.; F. E. Schnapp Die Sozialstaatsklausel - Beschwörungsformel oder Rechtsprinzip?, SGb 2000, 341 ff.; D. Suhr Rechtsstaatlichkeit und Sozialstaatlichkeit, Der Staat 9 (1970), 67 ff.; Zacher Staatsziel (Fn. 21), § 28 Rn. 109 ff. sowie den von E. Forsthoff herausgegebenen Sammelband „Rechtsstaatlichkeit und Sozialstaatlichkeit“", 1968. 


\section{Soziale Gleichheit als Thema des sozialen Staatsziels}

Da rechtliche Gleichheit aber auch gerade soziale Ungleichheit erzeugt, bedarf es, um soziale Gleichheit zu erreichen, weiterer Maßnahmen. Angesprochen ist insoweit das von Art. 79 Abs. 3 GG erfasste ${ }^{100}$ soziale Staatsziel ${ }^{101}$. Dieses bezieht sich mit den zentralen und zeitlosen Leitideen „soziale Sicherheit“ und „sozialer Ausgleich“102 auf Freiheitsvoraussetzungen, ${ }^{103}$ indem es auf die ungleichen tatsächlichen Lebensverhältnisse und die realen Bedürfnisse und Fähigkeiten des Einzelnen blickt. ${ }^{104}$ Mit dem sozialen Staatsziel thematisiert das Grundgesetz Freiheitsvoraussetzungen ${ }^{105}$ und weist zugleich dem Staat die Aufgabe zu, die sozialen Voraussetzungen der Ausübung grundrechtlicher Freiheit zu gewährleisten. ${ }^{106}$ Das soziale Staatsziel ist darauf gerichtet, für jedermann die tatsächlichen Voraussetzungen der Freiheit zu schaffen; es dient der Schaffung materieller Verfassungsvoraussetzungen. ${ }^{107}$ Der grundrechtlichen Freiheit soll ein Mindestmaß an sozialer Sicherheit und der grundrechtlichen Gleichheit ein Mindestmaß an sozialer Gleichheit korrespondieren. ${ }^{108}$

100 Der Schutz des Sozialstaatsprinzips durch Art. 79 Abs. 3 GG entspricht nahezu einhelliger Ansicht; statt Vieler: Heinig Sozialstaat (Fn. 18), 12 m. Fn. 29; H.-J. Papier Grundrechte und Sozialordnung, in: Handbuch der Grundrechte, Bd. II (Fn. 5), § 30 Rn. 5. - M. Jestaedt Bundesstaat als Verfassungsprinzip, HStR II, 3. Aufl. 2004, § 29 Rn. 55 mit Fn. 294, sieht dagegen das sozialstaatliche Prinzip „zumindest auf der Grundlage einer am Willen des Verfassung(sgesetz)gebers orientierten Verfassungsauslegung“ nicht als gemäß Art. 79 Abs. 3, 3. Alt GG revisionsfest an; nach Wiederin Sozialstaatlichkeit (Fn. 75), 74f., zählt die Sozialstaatlichkeit „nicht zum ewigen Verfassungskern“. - Kritik an dieser Auffassung etwa bei: H. F. Zacher Diskussionsbeitrag, VVDStRL 64 (2005), 89; R. Gröschner Diskussionsbeitrag, ebd., $175 \mathrm{f}$.

101 Zum Charakter des Sozialstaatsprinzips als Staatszielbestimmung statt Vieler: P. Badura Der Sozialstaat, DÖV 1989, 491 (493f.); Papier Grundrechte und Sozialordnung (Fn. 100), § 30 Rn. 1 ff.

102 S. dazu: E. Denninger Das soziale Staatsziel - zwischen Recht und Politik, FS H.-P. Schneider, 2008, 57 (63 ff.); K.-P. Sommermann, in: v. Mangoldt/Klein/Starck (Fn. 63), Art. 20 Rn. 104.

103 Kirchhof Der demokratische Rechtsstaat (Fn. 83), § 221 Rn. 148; s. auch: Kingreen Sozialstaatsprinzip (Fn. 22), 133.

104 Isensee Grundrechtsvoraussetzungen (Fn. 68), § 115 Rn. 158.

105 Isensee Grundrechtsvoraussetzungen (Fn. 68), § 115 Rn. 158. - Zur Problematik der Abgrenzung von grundrechtlicher Schutzpflicht und Grundrechtsvoraussetzung: Heinig Sozialstaat (Fn. 18), 388ff.; Kingreen Sozialstaatsprinzip (Fn. 22), $131 \mathrm{ff}$.

106 J. Isensee Vertragsfreiheit im Griff der Grundrechte, FS Großfeld, 1999, 485 (512).

107 Häberle Grundrechte im Leistungsstaat (Fn. 94), 95.

108 Isensee Grundrechtsvoraussetzungen (Fn. 68), § 115 Rn. 158. 


\section{Soziale Gleichheit durch soziale Sicherheit}

Die fundamentalen Ziele des neuzeitlichen und bürgerlichen Rechtsstaats, dem Einzelnen physische Sicherheit zu bieten und ihn vor Eingriffen in Freiheit und Eigentum zu schützen, werden im Sozialstaat ergänzt und erweitert um das Ziel der sozialen Sicherheit ${ }^{109}$ gegenüber den wirtschaftlichen Risiken des gesellschaftlichen Daseins in einer freiheitlichen Wettbewerbsgesellschaft. ${ }^{110}$ Als Sozialstaat gewährleistet der Staat des Grundgesetzes durch soziale Sicherheit als Komplementärprinzip zur Marktwirtschaft Schutz vor den Risiken des Marktes und ermöglicht die effektive Ausübung der Freiheit unter den Bedingungen ungleicher gesellschaftlicher Machtverhältnisse. ${ }^{111}$

Angesichts der sich rasant wandelnden gesellschaftlichen Verhältnisse, von der demographischen Entwicklung über den technologischen Fortschritt bis hin zur Globalisierung, ${ }^{112}$ unterliegen die Systeme sozialer Sicherheit einem ständigen Veränderungs- und Anpassungsdruck. Das soziale Staatsziel wirkt insoweit als permanenter Konkretisierungsauftrag, ${ }^{113}$ der - selbst wenn der Gesetzgeber regelmäßig historisch bedingten Entwicklungspfaden ${ }^{114}$ folgt und folgen muss - durch Offenheit und Prozesshaftigkeit geprägt ist. ${ }^{115}$ War der Sozialstaat in den Anfangstagen auf den Schutz der Schwachen, historisch vor allem der Industriearbeiterschaft, gerichtet, so weitete sich sein Blickfeld in der Folgezeit aus auf nahezu sämtliche Gruppen der Gesellschaft. Heute steht nicht

${ }^{109}$ Zum Verfassungsprinzip „Sicherheit“ im Hinblick auf die soziale Sicherheit: R. Pitschas Die Zukunft der sozialen Sicherungssysteme, VVDStRL 64 (2005), 109 (113ff.); zur Entwicklung im 19. Jahrhundert: D. Grimm Die sozialgeschichtliche und verfassungsrechtliche Entwicklung zum Sozialstaat, in: P. Koslowski/P. Kreuzer/ R. Löw (Hrsg.) Chancen und Grenzen des Sozialstaats, 1983, 41 (44ff.).

${ }_{110}$ Vgl. J. Isensee Die alte Frage nach der Rechtfertigung des Staates, JZ 1999, 265 (271 f.).

111 Vgl. J. Isensee Die Grundrechte als Abwehrrecht und als staatliche Schutzpflicht, HStR V, 2. Aufl. 2000, § 111 Rn. 132.

112 Zur Bedeutung und zu den Folgen der Globalisierung für den Sozialstaat: Zacher Staatsziel (Fn. 21), § 28 Rn. 158 ff. - Allgemein zur demographischen, gesellschaftlichen und wirtschaftlichen Entwicklung und deren Bedeutung für die soziale Sicherheit: $R$. Hauser Zukunft des Sozialstaats, in: B. Baron v. Maydell/F. Ruland/U. Becker (Hrsg.) Sozialrechtshandbuch (SRH), 4. Aufl. 2008, § 5 Rn. $28 \mathrm{ff}$.

${ }_{113}$ R. Pitschas Die Gesundheitsreform 2007, GesR 2008, 64 (65); vgl. auch: R. Scholz/R. Pitschas Sozialstaat und Gleichheit, FS 25 Jahre Bundessozialgericht, Bd. 2, 1979, 627 (628).

${ }^{114}$ Zur Pfadabhängigkeit: U. Davy Pfadabhängigkeit in der sozialen Sicherheit, in: SDSRV 55 (2007), $103 \mathrm{ff}$.

${ }_{115}$ Zacher Staatsziel (Fn. 21), § 28 Rn. 83 ff. - Zur Offenheit des Sozialstaatsprinzips vgl. auch G. Haverkate Rechtsfragen des Leistungsstaats, 1983, $56 \mathrm{ff}$. 
mehr allein die Abwehr von Notlagen im Mittelpunkt, sondern zunehmend die Sicherstellung des Normalniveaus. Soziale Sicherheit bezieht sich auch und besonders auf die Vorsorge in den allen drohenden Wechselfällen des Lebens, nicht nur auf die soziale Entschädigung Einzelner ${ }^{116}$ oder auf die Fürsorge bei Hilfebedürftigkeit, selbst wenn der Gewährleistung des Existenzminimums nach wie vor besondere Bedeutung, gerade auch für die soziale Gleichheit, zukommt.

\section{a) Gewährleistung des Existenzminimums}

Die Pflicht zur Gewährleistung des Existenzminimums als Mindestinhalt sozialer Gleichheit folgt aus der Menschenwürdegarantie des Art. 1 Abs. 1 GG in Verbindung mit dem Sozialstaatsprinzip, ${ }^{117}$ wobei insbesondere das Grundrecht auf Leben und das Grundrecht der allgemeinen Handlungsfreiheit die Pflicht verstärken und ihr Richtung geben. Der Pflicht korrespondiert ein, für die Schweiz ${ }^{118}$ hinsichtlich der unerlässlichen Mittel zur Hilfe in Notlagen sogar ausdrücklich formuliertes, verfassungsrechtliches Recht des Bedürftigen. Dieses hat seine grundgesetzliche Geltungsbasis jedoch nicht primär in dem in erster Linie auf Ausgestaltung durch den Gesetzgeber gerichteten sozialen Staatsziel, sondern in den Grundrechten. ${ }^{119}$

${ }^{116}$ Zur sozialen Entschädigung: F. Hase Soziales Entschädigungsrecht, in: Sozialrechtshandbuch (Fn. 112), § 26 Rn. 1 ff.; Zacher Staatsziel (Fn. 21), § 28 Rn. 44 f. - Zum Umfang des Schadensausgleichs bei der Wiedergutmachung von Vermögensschäden durch die Bodenreformen auf dem Gebiet der ehemaligen DDR zwischen 1945 und 1949: BVerfGE 102, 254 (297ff.).

117 Vgl. etwa: BVerfGE 82, 60 (85); 113, 88 (108f.); H. Dreier, in: ders. (Fn. 21), Art. 1 Rn. 158; M. Herdegen, in: Maunz/Dürig (Fn. 20), Art. 1 Rn. 114; W. Höfling, in: M. Sachs (Hrsg.) Grundgesetz, 4. Aufl. 2007, Art. 1 Rn. 31 f.; V. Neumann Diskussionsbeitrag, VVDStRL 64 (2005), $101 \mathrm{f}$; G. Robbers, in: D. Umbach/ T. Clemens (Hrsg.) Grundgesetz, Bd. I, 2002, Art. 1 Rn. 58f.; zum steuerfrei zu belassenden Existenzminimum bei der Einkommensbesteuerung vgl. nur: BVerfG, NJW 2008, 1868 (1871f.) mwN. - Allein auf das Sozialstaatsprinzip abstellend dagegen: C. Enders, in: K. H. Friauf/W. Höfling (Hrsg.) Berliner Kommentar zum Grundgesetz, Stand des Gesamtwerks: 2008, Art. 1 Rn. 114ff.; ders. Sozialstaatlichkeit im Spannungsfeld von Eigenverantwortung und Fürsorge, VVDStRL 64 (2005), 7 (39f.).

$118 \mathrm{Zu}$ Art. 12 der schweizerischen Bundesverfassung: M. Bigler-Eggenberger, in: B. Ehrenzeller/P. Mastronardi/R. J. Schweizer/K. A. Vallender (Hrsg.) Die schweizerische Bundesverfassung, 2. Aufl. 2008, Art. 12 Rn. 1 ff.; A. Epiney/B. Waldmann Soziale Grundrechte und soziale Zielsetzungen, in: D. Merten/H.-J. Papier (Hrsg.) Handbuch der Grundrechte, Bd. VII/2, 2007, § 224 Rn. 9 ff. - Überblick über den verfassungsrechtlichen Schutz des Existenzminimums in einzelnen europäischen Staaten bei R. Hofmann/P. Holländer/F. Merli/E. Wiederin (Hrsg.) Armut und Verfassung, 1998.

119 Isensee Grundrechtsvoraussetzungen (Fn. 68), § 115 Rn. 160. 
Originäre Ansprüche ${ }^{120}$ auf bestimmte Maßnahmen zur Herstellung sozialer Gleichheit lassen sich, auch jenseits des Existenzminimums, grundsätzlich nicht allein aus dem Sozialstaatsprinzip oder in Verbindung mit dem allgemeinen Gleichheitssatz ${ }^{121}$ - anders als derivative Ansprüche ${ }^{122}$ - begründen. ${ }^{123}$ Vielmehr können diese allenfalls von den jeweiligen Grundrechten her entwickelt werden ${ }^{124}$ und zielen dann regelmäßig auch nur auf die (gesetzliche ${ }^{125}$ ) Gewährleistung von Mindeststandards. ${ }^{126}$ Im sog. Nikolaus-Beschluss hat das Bundesverfassungsgericht ${ }^{127}$ für den Fall einer lebensbedrohlichen oder regelmäßig tödlichen

${ }^{120}$ Zur Terminologie: Murswiek Grundrechte als Teilhaberechte (Fn. 80), § 112 Rn. 5 ff. - Grundlegend zu der Frage von Grundrechten als Leistungsrechten die Referate von $W$. Martens und $P$. Häberle, Grundrechte im Leistungsstaat, auf der Staatsrechtslehrertagung von 1971 (VVDStRL 30 (1972), 7 ff., $44 \mathrm{ff}$.).

121 Vgl. W. Heun Freiheit und Gleichheit, in: Handbuch der Grundrechte, Bd. II (Fn. 5), § 34 Rn. 49; S. Muckel Sozialrecht, 2. Aufl. 2007, § 6 Rn. 18; Rüfner Bonner Kommentar (Fn. 54), Art. 3 Abs. 1 Rn. 65; R. Zippelius Der Gleichheitssatz, VVDStRL 47 (1989), 7 (15). - Allgemein zum Ausschluss originärer Leistungsrechte und zur Möglichkeit derivativer Teilhaberechte aus Art. 3 GG: Murswiek Grundrechte als Teilhaberechte (Fn. 80), § 112 Rn. 68 ff.; s. auch: H. A. Haller Die Verrechnung von Vor- und Nachteilen im Rahmen von Art. 3 Abs. 1 GG, 2007, 153 ff., 290.

122 Vgl. S. Huster, in: Berliner Kommentar (Fn. 117), Art. 3 Rn. 108; V. Neumann Sozialstaatsprinzip und Grundrechtsdogmatik, DVB1. 1997, 92 (97).

123 Vgl. statt Vieler: E. Grabitz Freiheit und Verfassungsrecht, 1976, 42ff.; M. Sachs, in: ders. (Fn. 117), Art. 20 Rn. 50. - Die schweizerische Bundesverfassung schließt in Art. 41 Abs. 4 ausdrücklich unmittelbare Ansprüche auf staatliche Leistungen aus den Sozialzielen aus; s. dazu: Bigler-Eggenberger (Fn. 118), Art. 41 Rn. 94 ff.; Epiney/Waldmann Soziale Grundrechte (Fn. 118), § 224 Rn. 60.

124 Zur Problematik der Begründung von Leistungsansprüchen aus Grundrechten aus neuerer Zeit mwN auf die reichhaltige Literatur zu diesem Thema: Heinig Sozialstaat (Fn. 18), $359 \mathrm{ff}$; R R̈fner Leistungsrechte (Fn. 95), § 40 Rn. $42 \mathrm{ff}$.

125 Zur Funktion, Bedeutung und Notwendigkeit gesetzlicher Regelungen in diesem Kontext und dem weiten Spielraum des Gesetzgebers: Rüfner Leistungsrechte (Fn. 95), $\S 40 \mathrm{Rn} .53 \mathrm{f}$.

126 S. dazu: R. Breuer Grundrechte als Anspruchsnormen, FG 25 Jahre Bundesverwaltungsgericht, 1978, 89 ff.; Rüfner Leistungsrechte (Fn. 95), § 40 Rn. 21.

127 Beschluss vom 6. Dezember 2005: BVerfGE 115, 25 ff. - Zu dieser Entscheidung: BSG, Breithaupt 2007, 366ff.; P. Axer Kontinuität durch Konsequenz in der Sozialversicherung, FS Isensee, 2007, 965 (972ff.); H.-U. Dettling Grundrechte, neue Behandlungsmethoden und Grenzen der Rationierung in der GKV, GesR 2006, $97 \mathrm{ff}$.; R. Francke/D. Hart Die Leistungspflicht der gesetzlichen Krankenversicherung für Heilversuche, MedR 2006, 131 ff.; H. M. Heinig Hüter der Wohltaten?, NVwZ 2006, 771 ff.; S. Huster Urteilsanmerkung, JZ 2006, 466 ff.; T. Kingreen Verfassungsrechtliche Grenzen der Rechtsetzungsbefugnis des Gemeinsamen Bundesausschusses im Gesundheitsrecht, NJW 2006, 877 ff.; M. von Wulffen Rechtsprechung des Bundessozialgerichts zu noch nicht anerkannten Behandlungsmethoden, GesR 2006, $385 \mathrm{ff}$. 
Krankheit im Wege einer „grundrechtsorientierten Auslegung“128 krankenversicherungsrechtlicher Vorschriften - jedoch unter konstruktiv nicht überzeugender Heranziehung des Schutzpflichtengedankens ${ }^{129}$ aus Art. 2 Abs. 1 GG iVm dem Sozialstaatsprinzip und Art. 2 Abs. 2 GG letztlich allerdings einen sehr weitgehenden verfassungsunmittelbaren Anspruch auf vom Gemeinsamen Bundesausschuss nicht zugelassene Untersuchungs- und Behandlungsmethoden kreiert. ${ }^{130}$ Dessen Konkretisierung und Umsetzung beschäftigt die Sozialgerichtsbarkeit etwa anhand von Elektroakupunktur oder natriumarmem Mineralwasser bis heute ${ }^{131}$ und illustriert zugleich die weit reichenden Folgen gerichtlich geschaffener verfassungsunmittelbarer Ansprüche, selbst wenn sich diese nur auf soziale Mindeststandards beziehen.

Ist die staatliche Pflicht zur Gewährleistung des Existenzminimums inzwischen allgemein anerkannt, so bereitet die Bestimmung von Inhalt und Umfang im Einzelnen nach wie vor Schwierigkeiten. ${ }^{132}$ Aus der grundgesetzlichen Garantie der Menschenwürde folgt die Pflicht, die Lebensumstände so zu sichern, dass ein autonomes und selbstbestimmtes Leben noch möglich ist. ${ }^{133} \mathrm{Zu}$ gewährleisten ist damit das für die physische Existenz und die soziokulturelle Entfaltung Notwendige. ${ }^{134}$

\footnotetext{
128 BVerfGE 115, 25 (45).

${ }^{129}$ Kritik an der Heranziehung der Schutzpflichtenkonstruktion etwa bei: Heinig Hüter der Wohltaten (Fn. 127), 773.

130 Axer Kontinuität durch Konsequenz (Fn. 127), 974f.; Huster Urteilsanmerkung (Fn. 127), 466 (468); ders. Soziale Sicherung als Zukunftsbewältigung, SDSRV 35 (2007), 15 (21 ff.); aA R. Schmidt-De Caluwe Urteilsanmerkung, SGb 2006, 619 (620f.). Nach Ansicht des Bundessozialgerichts (BSGE 97, 190 (196)) verlangt Art. 2 GG „eine verfassungskonforme Auslegung nur derjenigen Normen des SGB V, die einem verfassungsrechtlich begründeten Anspruch auf eine bestimmte Versorgung der Versicherten entgegenstehen".

131 S. etwa: BSGE 97, $190 \mathrm{ff}$; E. Hauck Gestaltung des Leistungsrechts der gesetzlichen Krankenversicherung durch das Grundgesetz?, NJW 2007, $1320 \mathrm{ff}$; U. Wenner Grenzen der Leistungspflicht der Kassen für nicht anerkannte Behandlungsverfahren und nicht zugelassene Arzneimittel, SozSich 2007, 75 ff., jeweils mwN. - Zur Elektroakupunktur: BSG, B. v. 9. 11. 2006, B 10 KR 3/06 B; zum natriumarmen Mineralwasser: BSG, B. v. 19. 6. 2006, B 1 KR 18/06 B.

${ }^{132}$ Dazu, jeweils mwN: W. G. Leisner Existenzsicherung im Öffentlichen Recht, 2007, $98 \mathrm{ff} ., 218 \mathrm{ff}$; J. Martínez Soria Das Recht auf Sicherung des Existenzminimums, JZ 1995, $644 \mathrm{ff}$.; V. Neumann Menschenwürde und Existenzminimum, NVwZ 1995, $426 \mathrm{ff}$; M. Wallerath Zur Dogmatik eines Rechts auf Sicherung des Existenzminimums, JZ 2008, $157 \mathrm{ff}$.

133 Heinig Sozialstaat (Fn. 18), 348.

${ }^{134}$ Zum soziokulturellen Existenzminimum mit weiteren Nachweisen: W. Spellbrink, in: W. Eicher/W. Spellbrink (Hrsg.) SGB II, Grundsicherung für Arbeitssuchende, 2. Aufl. 2008, § 20 Rn. 48.
} 
Was dazu zählt, ergibt sich aus einem Vergleich, der sich an den allgemeinen Lebensbedingungen des gesellschaftlichen Umfelds orientiert, damit räumlich-zeitlich relativ ${ }^{135}$ ist, und von dem Zweck geleitet wird, Ausgrenzungen und Stigmatisierungen zu vermeiden. ${ }^{136}$ Letztlich hängt damit die Leistungshöhe von dem in der jeweiligen Gesellschaft für erforderlich angesehenen Mindestbedarf ${ }^{137} \mathrm{ab} .{ }^{138}$

Das Existenzminimum gewährt nur eine begrenzte Gleichstellung und ermöglicht nicht jede beliebige Grundrechtsausübung. ${ }^{139}$ Auf die jeweilige Person bezogen, soll es den individuellen Verlust an Autonomie kompensieren und - auch durch Fordern ${ }^{140}$ - Autonomie wiederherstellen. ${ }^{141} \mathrm{Maßnahmen}$ zur Sicherung des Existenzminimums zielen damit nicht auf selbstzweckhafte Egalität im Minimum, sondern sind individuell und bedarfsgerecht ${ }^{142}$ festzusetzen, selbst wenn Pauschalierungen schon aus Gründen des Gesetzesvollzugs geboten sind. Allerdings hat das Recht der Grundsicherung für Arbeitssuchende im SGB II die Pau-

${ }^{135}$ Zur Relativität von Sozialhilfeleistungen sowie anderen staatlichen Leistungen, etwa den Renten: $U$. Häde Zum verfassungsrechtlichen Anspruch auf Erhöhung öffentlich-rechtlicher Geldleistungen, DÖV 2008, 613 (619).

136 BSGE 97, 265 (276); Wallerath Sicherung des Existenzminimums (Fn. 132), 165.

137 Zur Abhängigkeit der Mindestbedarfe von den jeweiligen „Gewohnheiten des Landes": A. Smith Der Wohlstand der Nationen (1776), deutsche Ausgabe hrsg. v. H. C. Recktenwald, 1974, Fünftes Buch, Kapitel 2, 4. Abschnitt, 747.

138 Diesen hat der Gesetzgeber entweder selbst oder die Verwaltung aufgrund hinreichend bestimmter gesetzlicher Vorgaben realitätsgerecht zu ermitteln. Zur verfassungsrechtlichen Zulässigkeit der Festsetzung des Regelsatzes nach $§ 20$ Abs. 2 SGB II vgl. etwa: BSGE 97, 265 (276ff.) mwN sowie die Entscheidung der 3. Kammer des 1. Senats des Bundesverfassungsgerichts (SGb 2008, 409f.), durch die eine Verfassungsbeschwerde wegen das Existenzminimum unterschreitender Gewährung von Arbeitslosengeld II mangels hinreichender Substantiierung für unzulässig erklärt wurde; kritisch zu dieser Entscheidung: K.-J. Bieback Entscheidungsanmerkung, SGb 2008, $410 \mathrm{ff}$. Zur realitätsgerechten Bemessung des tatsächlichen Bedarfs bei der Bestimmung der Steuerfreiheit des Existenzminimums: BVerfG NJW 2008, 1868 (1871 ff.).

139 S. dazu: Heinig Sozialstaat (Fn. 18), 394 ff.; Rüfner Leistungsrechte (Fn. 95), § 40 Rn. $66 \mathrm{ff}$.

140 Zum Grundsatz des Forderns und Förderns im SGB II und dessen Umsetzung jüngst mwN: W. Spellbrink Gelingt durch die neuen Instrumente des SGB II die Integration der Langzeitarbeitslosen in den Arbeitsmarkt?, SGb 2008, $445 \mathrm{ff}$.

141 Zum Ziel der Sozialstaatlichkeit, tatsächliche Autonomie herzustellen und zu sichern, allgemein: P. Unruh Kant - Menschenwürde - Sozialstaat, FS Starck, 2007, 133 (144ff.).

${ }^{142} \mathrm{Zu}$ den Inhalten und zur Bedeutung von Bedarfsgerechtigkeit im Sozialrecht H. F. Zacher Verfassung und Sozialrecht, FS Dürig, 1990, 67 (78ff.), dort auch zur Leistungsgerechtigkeit und zur Besitzstandsgerechtigkeit. 
schalierung im Vergleich zum Sozialhilferecht des SGB XII sehr und letztlich auch zu weit getrieben. ${ }^{143}$

In Zusammenhang mit der Sicherung des Existenzminimums steht die Diskussion um gesetzliche Mindestlöhne, die schon aufgrund ihrer tatsächlichen Auswirkungen auf Wachstum und Beschäftigung höchst umstritten sind. ${ }^{144}$ Doch dienen Mindestlöhne nicht nur der Existenzsicherung, wenn sie, in ihrer Höhe das Existenzminimum übersteigend, ${ }^{145}$ eher eine angemessene Teilhabe am gesellschaftlichen Leben bezwecken ${ }^{146}$ oder zur Vermeidung von Lohndumping selbst gegenüber Tariflöhnen eine Untergrenze festsetzen bzw. angesichts sinkender Tarifbindung das Tarifvertragssystem insgesamt stärken und schützen sollen. Staatliche Mindestlöhne, die je nach Höhe und Ausgestaltung, etwa wenn konkurrierende Tarifverträge verdrängt werden, mit dem Grundrecht der Koalitionsfreiheit ${ }^{147}$ und überdies mit der durch Art. 12 GG geschützten Arbeitsvertragsfreiheit ${ }^{148}$ in Konflikt geraten können, sind

143 Zu dieser Problematik: Spellbrink, in: Eicher/Spellbrink (Fn. 134), § 20 Rn. 35 ff., 44 f.; S. Knickrehm ebd., § 5 Rn. 16ff.; s. auch BSGE 97, 242 (248ff.) zur Gewährung der Kosten, die erforderlich sind, um einem geschiedenen Elternteil den Umgang mit seinen minderjährigen Kindern zu ermöglichen.

144 Vgl. etwa die Nachweise auf den Streitstand bei M. Fischer Gesetzlicher Mindestlohn, sozialrechtlich garantiertes Mindesteinkommen und Grundgesetz, ZG 2008, 32 (41); C. Schäfer Entwicklungsland Deutschland: Niedriglohnregulierung ohne Mindestlohn, in: K.-J. Bieback/T. Dieterich/P. Hanau/E. Kocher/C. Schäfer, Tarifgestützte Mindestlöhne, 2008, 9ff.; U. van Suntum/S. Gundel „Ein Arbeiter ist seines Lohnes wert“, 2007, $10 \mathrm{ff}$., sowie die Diskussion „Pro \& Contra“ zwischen dem Bundesminister für Arbeit und Soziales O. Scholz und dem Arbeitgeberpräsidenten D. Hundt in ZRP 2008, 167. - Begründung und Rechtfertigung eines Mindestlohnes in den Gesetzesentwürfen zum Ersten Gesetz zur Änderung des Gesetzes über die Festsetzung von Mindestarbeitsbedingungen (BR-Drucks. 541/08) sowie zum Arbeitnehmer-Entsendegesetz (BRDrucks. 542/08).

145 Nach Fischer (Gesetzlicher Mindestlohn (Fn. 144), 32 ff.) würde bei einem Alleinstehenden ein Mindestlohn von 4,80 Euro umgerechnet dem Niveau der Leistungen nach dem SGB II entsprechen, gefordert wird aber zumeist ein Mindestlohn von 7,50 Euro und höher.

146 Der Gesetzesantrag des Landes Rheinland-Pfalz auf Erlass eines Mindestlohngesetzes (BR-Drucks. 622/07) sieht nach $\S 1$ des Entwurfs die Funktion des Mindestlohns darin, ein die Existenz sicherndes Einkommen zu gewährleisten und eine angemessene Teilhabe am gesellschaftlichen Leben zu ermöglichen.

147 Wesentlicher Inhalt der Koalitionsfreiheit ist gerade die Festsetzung des Arbeitsentgelts durch die Tarifvertragsparteien; s. nur BVerfGE 116, 202 (219).

148 Vgl. dazu: Fischer Gesetzlicher Mindestlohn (Fn. 144), 38 ff.; ders. Gesetzlicher Mindestlohn - Verstoß gegen die Koalitionsfreiheit? ZRP 2007, 20 ff.; verfassungsrechtliche Zulässigkeit dagegen bejahend bzw. entsprechende Wege aufzeigend: Entwurf eines Gesetzes über die Festlegung des Mindestlohns, BR-Drucks. 622/07; K.-J. Bieback Rechtliche Probleme von Mindestlöhnen, insbesondere nach dem Arbeitnehmer- 
nicht durch das soziale Staatsziel, die Menschenwürdegarantie ${ }^{149}$ oder die Berufsfreiheit ${ }^{150}$ verfassungsrechtlich geboten, wenn der Staat das Existenzminimum anderweitig sichert, etwa durch Aufstocken der Löhne, ${ }^{151}$ und ein auffälliges Missverhältnis von Leistung und Gegenleistung mit Hilfe straf- und zivilrechtlicher Instrumente, etwa als Lohnwucher über $\S 138$ BGB, 152 verhindert werden kann. ${ }^{153}$ Bevor durch flächendeckende staatliche Mindestlöhne das mitgliederbezogene Tarifvertragssystem von seinen Grundpfeilern gelöst und der Arbeitgeber zu existenzsichernden Leistungen gegenüber seinen Arbeitnehmern herangezogen wird, erscheint es angemessener, ein auffälliges Missverhältnis zwischen Arbeitsleistung und Entgelt, das es auch jenseits von

Entsendegesetz, RdA 2000, 207 ff.; E. Kocher Mindestlöhne und Tarifautonomie, NZA 2007, $600 \mathrm{ff}$;; zur Gesamtproblematik s. auch: K.-J. Bieback/T. Dieterich/P. Hanau/ E. Kocher/C. Schäfer Tarifgestützte Mindestlöhne, 2008; A. Engels Verfassungsrechtliche Determinanten staatlicher Lohnpolitik, JZ 2008, $490 \mathrm{ff}$;; G. Peter Gesetzlicher Mindestlohn, 1995; speziell zur Festsetzung von Mindestentgelttarifverträgen im Bereich der Briefdienstleistungen: VG Berlin, NVwZ 2008, 804 ff.; K.-J. Bieback Die Wirkung von Mindestentgelttarifverträgen gegenüber konkurrierenden Tarifverträgen, AuR 2008, 234 ff.; J. A. Kämmerer/G. Thüsing Tariferstreckung in der Postdienstleistungsbranche, 2007; U. Preis/S. Greiner Rechtsgutachten zur Allgemeinverbindlichkeitserklärung ( 55 TVG) oder Geltungserstreckung ( 1 Abs. 3a AEntG) eines Mindestlohn-Tarifvertrags in der Postdienstleistungsbranche, 2007.

${ }^{149}$ Nach K. Kruis Sondervotum zum Urteil zur Pflichtarbeit im Strafvollzug, BVerfGE 98, 217, wird der Mensch in seiner existentiellen Befindlichkeit in Frage gestellt, wenn er einer Ordnung ausgesetzt ist, in der für ihn der Zusammenhang zwischen abverlangter Arbeit und angemessenem (gerechtem) Lohn prinzipiell aufgehoben ist.

150 Von einer Schutzpflicht aus Art. 12 GG im Kontext der Mindestlohnproblematik spricht T. Dieterich Verfassungsmäßigkeit tarifgestützter Mindestlöhne bei Zeitarbeit, in: Tarifgestützte Mindestlöhne (Fn. 144), 103 (117f.). - Allgemein zur Frage grundrechtlicher Schutzpflichten im Hinblick auf privatrechtliche Verträge mwN: Isensee Vertragsfreiheit (Fn. 106), $497 \mathrm{f}$., $500 \mathrm{ff}$.

${ }^{151}$ Zur Regelung in $\S 30$ SGB II, die dem Grundsatz Rechnung tragen soll, dass der Erwerbstätige mehr Geld zur Verfügung haben soll als derjenige, der trotz Erwerbsfähigkeit nicht arbeitet: C. Mecke, in: Eicher/Spellbrink (Fn. 134), § 30 Rn. 4 ff.; Spellbrink Integration der Langzeitarbeitslosen (Fn. 140), 451.

${ }_{152}$ Zum Lohnwucher und dessen Behandlung nach § 138 B GB: K. Bepler Problematische Arbeitsverhältnisse und Mindestlohn, FS Richardi, 2007, 189 (191 ff.); M. Henssler/U. Sittard Flexibler Mindestlohn durch Konkretisierung des Sittenwidrigkeitstatbestands, RdA 2007, 159 ff.; H. Heinrichs, in: Palandt BGB, 67. Aufl. 2008, §138 BGB Rn. 79; zur strafrechtlichen Sanktionierung nach § 291 StGB: H. Tröndle/T. Fischer Strafgesetzbuch, 55. Aufl. 2008, § 291 StGB Rn. 7.

${ }^{153}$ Eine andere Frage ist es, ob und inwieweit niedrige Löhne Frauen gegenüber Männern im Niedriglohnsektor benachteiligen und sich als Verstoß gegen Art. 3 Abs. 2 S. 2 GG erweisen. Zu diesem Problemkreis: Bieback Probleme von Mindestlöhnen (Fn. 148), 209; Peter Gesetzlicher Mindestlohn (Fn. 148), $278 \mathrm{ff.}$ 
Hungerlöhnen geben kann, individualarbeitsrechtlich - ggf. durch eine entsprechende gesetzliche Konkretisierung des Lohnwuchers - zu sanktionieren und die Existenzsicherung über staatliche Zuschüsse zu gewährleisten. ${ }^{154}$ Der Staat gerät in verfassungsrechtliche Schwierigkeiten, wenn er flächendeckend marktwirtschaftlich vereinbarte Entgelte korrigieren und den „wahren Wert" einer Arbeitsleistung festsetzen will. Dies gilt auch dann, wenn er hohe Managergehälter - warum dann nicht auch die Gehälter von Fußballspielern? - deckeln will.

\section{b) Soziale Vorsorge}

Soziale Sicherheit wird für weite Teile der Bevölkerung durch die Sozialversicherung verwirklicht. ${ }^{155}$ Aufgrund zahlreicher Leistungseinschränkungen tendiert die Sozialversicherung in einzelnen Zweigen allerdings immer stärker zu einer Art Grund- und Mindestsicherung, wenn etwa Leistungen in der gesetzlichen Krankenversicherung auf das medizinisch Notwendige und Wirtschaftliche begrenzt sind und es einer Zulassung durch den Gemeinsamen Bundesausschuss bedarf. Selbst wenn das Bundesverfassungsgericht zu Recht betont, dass die Sozialversicherung im Grundsatz darauf ziele, einen über dem Sozialhilfeniveau liegenden Lebensstandard zu sichern, ${ }^{156}$ lässt sich in der Kranken- oder Pflegeversicherung ein höheres Leistungsniveau in der Regelversorgung kaum noch feststellen. ${ }^{157}$

Allerdings ist auch eine gegenläufige Entwicklung hin zu mehr Ausdifferenzierung und damit Ungleichheit zu beobachten. In der gesetzlichen Krankenversicherung wurden etwa durch das GKV-Wettbewerbsstärkungsgesetz 2007 in verfassungs- und europarechtlich bedenklicher

\footnotetext{
154 Vgl. dazu Henssler/Sittard Flexibler Mindestlohn (Fn. 152), 163 ff., die auf einen Vorschlag für eine gesetzliche Regelung hinweisen, nach dem bei einem auffälligen Wertmissverhältnis zwischen der Arbeitsleistung und dem vereinbarten Entgelt unter Berücksichtigung der sonstigen Vertragsbedingungen die Entgeltvereinbarung unwirksam sein soll. Ein auffälliges Wertmissverhältnis ist danach im Zweifel dann anzunehmen, wenn die Vergütung nicht mindestens zwei Drittel des am (regelmäßigen) Arbeitsort für die Arbeitsleistung üblichen Entgelts erreicht.

155 Nach BVerfGE 113, 167 (215) ist das Sozialversicherungsrecht eines der wichtigsten Instrumente staatlicher Sozialpolitik und gerade der Schutz in Fällen von Krankheit eine der Grundaufgaben des Staates.

156 BVerfG NJW 2008, 1868 (1872); zu dieser Entscheidung vgl. U. Wenner Beiträge zur Kranken- und Pflegeversicherung müssen besser von der Steuer absetzbar sein, SozSich 2008, $149 \mathrm{ff}$.

157 V. Neumann Das medizinische Existenzminimum, NZS 2006, 393 (394ff.) - Unterschiedliche Leistungen sind etwa beim Krankengeld vorgesehen.
} 
Weise ${ }^{158}$ Wahlleistungen ermöglicht. In der Rentenversicherung setzt sich die Ungleichheit des Arbeitseinkommens traditionell in den Leistungen - obwohl auch hier Einebnungstendenzen erkennbar sind - aufgrund der zu den Konstitutionsprinzipien der Rentenversicherung gehörenden Äquivalenz fort: Wer viel und lange eingezahlt hat, soll höhere Leistungen erhalten. ${ }^{159}$ Der den Ansprüchen und Anwartschaften gerade in der Rentenversicherung zukommende, durch die Gebote der Folgerichtigkeit und Konsequenz $z^{160}$ verstärkte Eigentumsschutz ${ }^{161}$ fordert grundsätzlich eine leistungsgerechte Differenzierung bei gleichzeitiger Absage an eine Bedarfsnivellierung ${ }^{162}$. Selbst wenn eine allgemeine Absenkung der Renten, sei es durch eine veränderte Rentenformel oder verlängerte Lebensarbeitszeiten, verfassungsrechtlich möglich ist und sich das Bundesverfassungsgericht bislang überhaupt zurückhaltend gezeigt hat, dem Reformgesetzgeber strenge Grenzen zu ziehen, ${ }^{163}$ schützen die Grundrechte des Versicherten vor einer mit dem Gedanken sozialer Gleichheit begründeten Einebnung des Rentenniveaus und der Einführung einer pauschalen Einheitsrente. ${ }^{164}$ Es wäre verfassungswid-

$158 \S 53$ SGB V. Verfassungs- und europarechtliche Kritik etwa bei: J. Isensee Wahltarif „Krankenhauskomfort“, NZS 2007, 449 ff.; P. M. Huber/S. Storr Die Wahltarife im SGB V, 2008.

159 P. Kirchhof Gerechtigkeit im sozialen Rechtsstaat, Zur Debatte 2000, 1 (5).

160 S. dazu, jeweils mwN: Axer Kontinuität durch Konsequenz (Fn. 127), 972 ff.; Papier Grundrechte und Sozialordnung (Fn. 100), § 30 Rn. $59 \mathrm{ff}$.

161 Zum Eigentumsschutz sozialversicherungsrechtlicher Anwartschaften und Ansprüche: E. Gurlit Die Reform der Rentenversicherung im Lichte der Eigentumsgarantie des Artikels 14 Grundgesetz, VSSR 2005, 45 (52ff.); A. Lenze Staatsbürgerversicherung und Verfassung, 2005, $41 \mathrm{ff}$; Muckel Sozialrecht (Fn. 121), § 6 Rn. 12ff.; V. Neumann Der Grundrechtsschutz von Sozialleistungen in Zeiten der Finanznot, NZS 1998, 401 ff.; P. Sonnevend Eigentumsschutz und Sozialversicherung, 2008; H.-J. Papier Der Einfluss des Verfassungsrechts auf das Sozialrecht, in: Sozialrechtshandbuch (Fn. 112), § 3 Rn. 41 ff.; Kritik an der verfassungsgerichtlichen Rechtsprechung zum Eigentumsschutz etwa bei: $O$. Depenheuer Wie sicher ist verfassungsrechtlich die Rente?, AöR 120 (1995), $417 \mathrm{ff}$.

162 Isensee Sozialstaat in der Wirtschaftskrise (Fn. 50), 380.

163 S. dazu: R. Pitschas Verfassungsvoraussetzungen für die Entstaatlichung der gesetzlichen Rentenversicherung in Deutschland, FS Ruland, 2007, 99 (114ff.).

164 Vgl. Papier Zukunft des Sozialstaates (Fn. 51), 5, wonach dauerhafte „Null-Renditen“ oder gar Minuswerte, dergestalt, dass die Rentenzahlungen bei weitem nicht mehr ausreichen, um das vom betroffenen Beitragszahler „,investierte Kapital“ zu verbrauchen, die Frage aufwerfen könnten, ob nicht die Grenze der verfassungsrechtlich unzulässigen, evidenten Disproportionalität von Leistung und Gegenleistung irgendwann erreicht bzw. überschritten wird. Skeptischer dagegen Pitschas Entstaatlichung der Rentenversicherung (Fn. 163), S. 114ff., der allerdings aus dem berufsgrundrechtlichen Schutz gesetzlicher Rentenansprüche ein Absinken des Renteneinkommens unter den Sozialhilfesatz als unzulässig ansieht. 
rig, wenn der Gesetzgeber die durch Zwangsbeiträge entstandenen Anwartschaften einheitlich etwa auf Sozialhilfeniveau absenken würde ${ }^{165}$ oder bedarfsabhängig ${ }^{166}$ zuteilte.

Unterschiedliche Leistungen kennzeichnen auch die Arbeitslosenversicherung aufgrund der Funktion des Arbeitslosengeldes als kurzfristige, am jeweiligen Arbeitsentgelt pauschaliert orientierte Entgeltersatzleistung. ${ }^{167}$ Als Risikoversicherung konzipiert, die allerdings das individuelle Arbeitsplatzrisiko bei der Beitragsbemessung gerade nicht berücksichtigt, 168 verlangt sie in ihrer jetzigen Form von Verfassungs wegen jedoch nicht, dass ein über Jahrzehnte einzahlender älterer Versicherter auch länger zum Arbeitslosengeldbezug berechtigt sein muss, ${ }^{169}$ gleichsam, als ob die Arbeitslosenversicherung eine Art Sparvertrag sei und das Risiko sich mindestens einmal verwirklicht haben müsse, damit sich die Beiträge rentierten. ${ }^{170}$

Soziale Ungleichheit entsteht auch aufgrund unterschiedlicher Vorsorgebiographien, weil der Sozialversicherung kein Vorsorgemonopol zukommt. So kann sich die Alterssicherung aus Leistungen berufsständischer Versorgungswerke ${ }^{171}$, aus der Beamtenversorgung, aus Betriebsrenten oder aus privater Vorsorge speisen und damit eine erhebliche Rang- und Statusvariierung bewirken. ${ }^{172}$ Die gesetzliche Krankenversi-

165 Vgl. dazu U. Wenner Rentenniveau und Grundgesetz, FS 50 Jahre BSG, 2004, $625 \mathrm{ff}$.

166 Vgl. BVerfGE 100, 138 (182). Danach muss in der gesetzlichen Rentenversicherung jedenfalls ein Leistungsrest erhalten bleiben, der den Zweck einer bedürftigkeitsunabhängigen Sicherung nach einem vollen Versicherungsleben erfüllt.

${ }^{167}$ Dazu: BVerfGE 102, 127 (143ff.); F. Hase Versicherungsprinzip und sozialer Ausgleich, 2000, 124ff. - Zu den Voraussetzungen und zur Berechnung des Arbeitslosengeldes nach den $\S \S 129 \mathrm{ff}$. SGB III vgl. statt Vieler: K.-J. Bieback Arbeitsförderung, in: Sozialrechtshandbuch (Fn. 112), § $21 \mathrm{Rn} .54 \mathrm{ff} . \mathrm{mwN}$.

168 BVerfGE 51, 115 (124f.).

${ }^{169} \mathrm{Zu}$ den unterschiedlichen Regelungen im Hinblick auf die Bezugsdauer: P. Coseriu/H. C. Jakob, in: B. Mutschler/R. Bartz/R. Schmidt-De Caluwe (Hrsg.) Sozialgesetzbuch III, 3. Aufl. 2008, § 127 SGB III Rn. 2 ff., 33 ff.

170 Vgl.: E. Eichenhofer Sozialreformen zwischen Vision und Wirklichkeit, NZS 2007, 57 (58 f); Heinig Sozialstaat (Fn. 18), 567 mit Fn. 80. - Verfassungsrechtliche Bedenken gegen die Kürzung der Leistungsbezugsdauer im Rahmen der Hartz III und Hartz IVReformen: W. Spellbrink Ist die Beitragspflicht in der gesetzlichen Arbeitslosenversicherung verfassungsrechtlich noch zu rechtfertigen? JZ 2004, $538 \mathrm{ff}$.; s. auch $U$. R. Mayer Geplante Verkürzung ist verfassungsrechtlich bedenklich, SozSich 2003, $239 \mathrm{ff}$.

$171 \mathrm{Zu}$ diesen $W$. Boecken Berufsständische Versorgungswerke, in: Sozialrechtshandbuch (Fn. 112), § 22 Rn. $1 \mathrm{ff}$.

172 Vgl. Eichenhofer Sozialreformen (Fn. 170), NZS 2007, 58, 60; zur Bedeutung staatlich geförderter privater Altersvorsorge und den damit zusammenhängenden verfassungsrechtlichen Fragen: Pitschas Entstaatlichung der Rentenversicherung (Fn. 163), 119. 
cherung umfasst trotz Einführung einer allgemeinen Versicherungspflicht durch das GKV-Wettbewerbsstärkungsgesetz bislang noch nicht alle Bevölkerungsschichten, so dass das Krankheitsrisiko auch privat und über die Beamtenbeihilfe abgesichert wird. Selbst wenn die private Krankenversicherung mit dem Basistarif ${ }^{173}$ und die Beamtenbeihilfe durch wirkungsgleiche Übertragung sozialversicherungsrechtlicher Reformen ${ }^{174}$ in Struktur und Inhalt der gesetzlichen Krankenversicherung immer stärker angeglichen werden und sich zudem vehement Forderungen nach einer allumfassenden, letztlich auf verfassungsrechtliche Widerstände stoßenden, staatlich organisierten und durchgeführten Bürgerversicherung ${ }^{175}$ erheben, unterscheiden sich die Leistungen der verschiedenen Vorsorgesysteme nach wie vor.

Gesetzliche Versuche, die Unterschiede zu beseitigen, müssen sich vor den das private Vermögen und die private Dispositionsfreiheit schützenden Grundrechten rechtfertigen lassen und mit der Garantie der hergebrachten Grundsätze des Berufsbeamtentums in Art. 33 Abs. 5 GG vereinbar sein. Eine auf den Gedanken sozialer Gleichheit gestützte Abschaffung der unterschiedlichen Systeme zugunsten einer staatlichen Einheitszwangsversicherung wäre angesichts des langen Zeitraums, in denen Anwartschaften entstehen, aus denen sich dann zum Teil jahrzehntelange Ansprüche ergeben, - wenn überhaupt ${ }^{176}$ - nur mit langen Übergangsfristen verfassungsrechtlich möglich. ${ }^{177}$

173 S. dazu: P. Axer Einbeziehung der PKV in die GKV, GesR 2008, 482 ff.; R. Pitschas Sozialrechtliche Regulierung des privatrechtlichen Versicherungswesens, FS Stober, 2008, 295 ff.; H. Sodan Verpflichtende Basistarife in der privaten Krankenversicherung als Verfassungsproblem, FS Isensee, 2007, $983 \mathrm{ff}$.; ders. Private Krankenversicherung und Gesundheitsreform 2007, 2. Aufl. 2007, 74ff.; A. Wallrabenstein/K. Bourcarde Verfassungsrechtliche Fragen an die Reform der PKV, MedR 2008, $415 \mathrm{ff}$.

174 Dazu: P. Axer Beihilfe unter dem Regime der Sozialversicherung, DVB1. 1997, $698 \mathrm{ff}$.

175 Verfassungsrechtliche Kritik etwa bei: P. Axer Verfassungsrechtliche Fragen einer Bürgerversicherung, GS Heinze, 2005, 1 ff.; F. Kirchhof Verfassungsrechtliche Probleme einer umfassenden Kranken- und Renten-,Bürgerversicherung“, NZS 2004, $1 \mathrm{ff}$;; J. Isensee „Bürgerversicherung“ im Koordinatenkreuz der Verfassung, NZS 2004, $393 \mathrm{ff}$.; dagegen ihre Zulässigkeit grundsätzlich bejahend etwa: K.-J. Bieback Sozial- und verfassungsrechtliche Aspekte der Bürgerversicherung, 2005.

176 Isensee Sozialstaat in der Wirtschaftskrise (Fn. 50), $382 \mathrm{f}$. Nicht ausgeschlossen ist es allerdings, zusätzliche private Sicherungssysteme zu errichten und diese bei der Festlegung von Inhalt und Umfang staatlicher Sicherungssysteme zu berücksichtigen, wie für die Rente geschehen; vgl. dazu Pitschas Entstaatlichung der Rentenversicherung (Fn. 163), $109 \mathrm{ff}$.

$177 \mathrm{Zu}$ den Grundsätzen und Inhalten sozialrechtlichen Übergangsrechts: J. Joussen Grundsätze sozialrechtlichen Übergangsrechts, SDSRV 55 (2007), 59ff.; s. auch: R. Giesen Vergangenheitsbezug sozialrechtlicher Normen, ebd., 33 ff. - Unabhängig von 
Ist die Forderung nach sozialer Gleichheit regelmäßig mit dem Wunsch nach sozialpolitischer Aktivität des Gesetzgebers verbunden, so zielt sie gerade auf dem Feld der sozialen Sicherheit auch auf Stetigkeit, Verlässlichkeit und Berechenbarkeit. Selbst wenn keine verfassungsrechtliche Systemgarantie für die jeweilige Organisation und Durchführung sozialer Leistungen existiert, ${ }^{178}$ streiten die Grundrechte sowie das Rechtsstaats- und das Sozialstaatsprinzip gegen einen abrupten und grundlegenden Systemwechsel, ${ }^{179}$ so dass die Einführung eines bedingungslosen Grundeinkommens oder eines solidarischen Bürgergeldes auch schon deshalb auf erhebliche rechtliche Schwierigkeiten stieße. ${ }^{180}$ Soziale Sicherheit braucht Kontinuität wie soziale Gleichheit nach einem schlüssigen und transparenten Konzept sozialer Sicherheit, nach Beständigkeit, auch unter Wahrung von Besitzstandsgerechtigkeit, ${ }^{181}$ verlangt. ${ }^{182}$ Doch zerstört gerade die augenblickliche Praxis mit ihren punktuellen, situationsbedingten und oftmals abrupten Änderungen $^{183}$ oder mit systembrechenden Vorschlägen diese Voraussetzungen. Die Pluralität und Komplexität sozialrechtlicher Leistungen, die unterschiedlichen Zuständigkeiten im Sozialen und das Gewirr von

den rechtlichen Schwierigkeiten stellt sich die Frage, ob eine auf Grundsicherung beschränkte Zwangsversicherung überhaupt mehr soziale Gleichheit bewirken würde, denn weitergehende Leistungen könnten und müssten durch private Zusatzversicherungen abgesichert werden, was angesichts unterschiedlicher finanzieller Möglichkeiten dazu wiederum zu sozialer Ungleichheit führen würde. S. dazu S. Huster Soziale Sicherung als Zukunftsbewältigung und -gestaltung, SDSRV 55 (2007), 15 (24ff.); zu den Problemen der Privatisierung von Sicherheit unter dem Gesichtspunkt der Gleichheit: Pitschas Frei - sozial - auch sicher? (Fn. 15), $288 \mathrm{ff}$.

178 So das Bundesverfassungsgericht in ständiger Rechtsprechung, vgl. nur: BVerfGE 113, 167 (219, 223).

179 Dazu: P. Axer Kontinuität durch Konsequenz (Fn. 127), 965 ff.; Pitschas Gesundheitsreform 2007 (Fn. 113), 68 ff.; zum rechts- und sozialstaatlichen Vertrauensschutz: Gröschner, in: Dreier (Fn. 21), Art. 20 (Sozialstaat) Rn. 44.

180 Zur Idee eines bedingungslosen Grundeinkommens oder solidarischen Bürgergeldes: D. Althaus Solidarisches Bürgergeld stärkt soziale Marktwirtschaft, KV 2008, 36 ff.; M. Borchard (Hrsg.) Das solidarische Bürgergeld - Analysen einer Reformidee, 2007; E. Eichenhofer Sozialversicherung und Grundeinkommen, APuZ 2007, Heft 51-52, $19 \mathrm{ff}$; I. Hobenleitner/T. Straubhaar Grundeinkommen und soziale Marktwirtschaft, ebd., $11 \mathrm{ff}$.

181 Zur Bedeutung der Besitzstandsgerechtigkeit im Sozialstaat: Zacher Verfassung und Sozialrecht (Fn. 142), 84.

182 Allgemein zur Bedeutung des Vertrauens in die Gewährleistung sozialer Sicherheit: Pitschas Entstaatlichung der Rentenversicherung (Fn. 163), $116 \mathrm{ff}$.

${ }^{183}$ Beispiele intransparenter Gesetzgebung bei $S$. Wolf Kuriositäten in der sozialversicherungsrechtlichen Gesetzgebung, SGb 2008, $516 \mathrm{ff}$. 
Ansprüchen in einem diffusen Geflecht von Besitzstands-, Bedarfs- und Leistungsgerechtigkeit überfordern die Betroffenen und erzeugen Unübersichtlichkeit, letztlich damit auch Ungleichheit.

\section{Soziale Gleichheit durch sozialen Ausgleich}

Soziale Gleichheit fordert neben sozialer Sicherheit einen sozialen Ausgleich, der in den Systemen sozialer Sicherheit selbst stattfindet, etwa im Wege der Umverteilung aufgrund nicht risiko-, sondern einkommensbezogener Krankenversicherungsbeiträge, durch einen trägerübergreifenden Risikostrukturausgleich ${ }^{184}$ oder die Möglichkeit der Familienversicherung. ${ }^{185}$ Der sozialstaatliche Handlungs- und Gestaltungsauftrag zum sozialen Ausgleich verpflichtet nach Ansicht des Bundesverfassungsgerichts den Gesetzgeber, für einen Ausgleich sozialer Gegensätze und für eine gerechte Sozialordnung zu sorgen. ${ }^{186}$ Die verfassungsgerichtliche Verbindung des Ziels einer gerechten Sozialordnung ${ }^{187}$ mit dem Gedanken des sozialen Ausgleichs ${ }^{188}$ verdeutlicht, dass sich auch verfassungsrechtlich soziale Gerechtigkeit ${ }^{189}$ wesentlich auf den Abbau sozialer Ungleichheit bezieht ${ }^{190}$ und die Herstellung sozialer Gleichheit fordert.

Grundsätzlich erfolgt der soziale Ausgleich auf zwei Wegen: Zum einen durch eine entsprechende Abgabenerhebung, etwa durch einen

${ }^{184}$ Zum Risikostrukturausgleich in der gesetzlichen Krankenversicherung, jeweils mwN: BVerfGE 113, 167 ff.; P. Axer Gesundheitswesen, HStR IV, 3. Aufl. 2006, § 95 Rn 15.

${ }^{185} \mathrm{Zu}$ Inhalt und Bedeutung des sozialen Ausgleichs jeweils mwN: BVerfGE 113, 167 (216ff.); H. Butzer Fremdlasten in der Sozialversicherung, 2001, $219 \mathrm{ff}$.; Hase Versicherungsprinzip und sozialer Ausgleich (Fn. 167), 254ff.; J. Isensee Umverteilung durch Sozialversicherungsbeiträge, 1973, $13 \mathrm{ff}$.

186 BVerfGE 5, 85 (198); 22, 180 (204); 35, 202 (235f.); 59, 231 (263); 69, 272 (314).

${ }^{187}$ Kritik an der Verwendung des Begriffs „gerechte Sozialordnung“ bei G. Roellecke, in: Umbach/Clemens (Fn. 117), Art. 20 Rn. 186.

${ }^{188} \mathrm{Zu}$ dieser Verbindung Gröschner, in: Dreier (Fn. 21), Art. 20 (Sozialstaat) Rn. 52.

${ }^{189}$ Nach Zacher Staatsziel (Fn. 21), § 28 Rn. 111, ist soziale Gerechtigkeit ein Ziel und eine Erfahrung, aber keine anwendbare Rechtsnorm. So wesentlich soziale Gerechtigkeit als letzter Grund des sozialen Rechtsstaats sei, so wenig sei sie ein verfassungsrechtlicher Tatbestand. - Zu den Problemen und Schwierigkeiten, mit Hilfe des Begriffs „soziale Gerechtigkeit“ den materiellen Gehalt des Sozialstaatsprinzips zu bestimmen: Sommermann, in: v. Mangoldt/Klein/Starck (Fn. 63), Art. 20 Rn. 104.

190 Zacher Staatsziel (Fn. 21), § 28 Rn. 53, weist zutreffend darauf hin, dass der gemeinsame Nenner der sozialen Bewegung primär auf Verbesserung der Lebensverhältnisse der „unteren Schichten“ gerichtet war, nicht dagegen auf Verschlechterung der Lebensverhältnisse der „oberen Schichten“. 
progressiven Einkommensteuertarif, ${ }^{191}$ wobei die progressive Ausgestaltung weder sozialstaatlich noch sonst verfassungsrechtlich zwingend ist, ${ }^{192}$ zum anderen durch die Gewährung von Leistungen. Beide Wege des sozialen Ausgleichs sind nicht beliebig begehbar und austauschbar, denn staatliches Nehmen und Geben sind schon als Eingriff und Leistungsgewährung in Voraussetzungen und Wirkung nicht gleichwertig. So kommt etwa im Hinblick auf das Familienexistenzminimum der Steuerverschonung Vorrang zu gegenüber sozialrechtlichen Leistungen, ${ }^{193}$ denn eine freiheitliche Rechtsordnung verlangt, zum Schutz des Familienexistenzminimums schon auf den Steuereingriff zu verzichten, anstatt zuerst abzuschöpfen, um dann umzuverteilen und wieder zuzuteilen. Zu Recht hat das Bundesverfassungsgericht jüngst auf einen Grundgedanken der Subsidiarität hingewiesen, dass nämlich der Eigenversorgung Vorrang vor staatlicher Fürsorge zukomme. 194

\section{Soziale Gleichheit als Chancengleichheit}

Dem freiheitlichen Sozialstaat ${ }^{195}$ des Grundgesetzes entspricht es, soziale Gleichheit durch Herstellung von Chancengleichheit ${ }^{196}$ im Sinne der Ermöglichung gleicher Startbedingungen und gleicher Entwicklungschancen aller zur Teilhabe am wirtschaftlichen und gesellschaftlichen Leben zu verwirklichen. Würden dagegen soziale Ungleichheiten durch permanente Leistungen und Zuteilungen zum Zwecke gleicher

${ }^{191}$ Kritik an der derzeitigen Ausgestaltung des progressiven Einkommensteuertarifs mwN: Kirchhof Steuern (Fn. 34) § 118 Rn. 271 ff.; allgemein zu Grund und Grenzen der Steuerprogression Lammers Steuerprogression (Fn. 34), $22 \mathrm{ff}$.

192 BVerfGE 115, 97 (117); anders noch BVerfGE 8, 51 (68f.). - S. dazu: Waldhoff Grundzüge des Finanzrechts (Fn. 34), § 116 Rn. 108.

${ }^{193}$ Vgl. P. Axer Die Familie zwischen Privatrecht, Sozialrecht und Steuerrecht, in: R. Mellinghoff (Hrsg.) Steuern im Sozialstaat, 2006, 175 (187f.).

194 BVerfG, NJW 2008, 1868 (1872).

195 Zum Begriff: Herzog, in: Maunz/Dürig (Fn. 20), Art. 20 VIII Rn. 34 ff.; Gröschner, in: Dreier (Fn. 21), Art. 20 (Sozialstaat) Rn. 1; Kingreen Sozialstaatsprinzip (Fn. 22), $121 \mathrm{ff}$; Zacher Staatsziel (Fn. 21), § 28 Rn. $25 \mathrm{ff}$.

196 Zum Begriff: Engels Chancengleichheit und Bundesstaatsprinzip (Fn. 62), $20 \mathrm{ff}$; aus schweizerischer Sicht: Müller Soziale Grundrechte (Fn. 95), $223 \mathrm{ff}$; B. Weber-Dürler Chancengleichheit und Rechtsgleichheit, FS Häfelin, 1989, 205 ff.; dies. Gleichheit, in: Handbuch der Grundrechte, Bd. VII/2 (Fn. 118), § 210 Rn. 49. - Kritisch zum Begriff im Rahmen des Gleichbehandlungsgrundsatzes: Herzog, in: Maunz/Dürig (Fn. 20), Art. 20 VIII Rn. 37 ff.; C. Starck, in: H. v. Mangoldt/F. Klein/C. Starck (Hrsg.) Grundgesetz, Bd. 1, 5. Aufl. 2005, Art. 3 Rn. 33; zu Inhalt und Bedeutung von Chancengleichheit im Prüfungsrecht, Parteienrecht, Prozessrecht und im Wirtschaftsrecht: Engels Chancengleichheit und Bundesstaatsprinzip (Fn. 62), $28 \mathrm{ff}$. 
Erfolgsverwirklichung ausgeglichen, führte die damit bewirkte Ergebnisgleichheit zu einer egalitären Gesellschaft, welche die individuelle und eigenverantwortliche Lebensgestaltung einebnete, damit Freiheit in ihr Gegenteil verkehrte. ${ }^{197}$ Dagegen wird durch die Gewährung von Chancengleichheit im Sinne gleicher Startbedingungen Freiheit vermittelt. ${ }^{198}$ Im Prinzip der Chancengleichheit verbinden sich Freiheit, Eigenverantwortung und Sozialstaatlichkeit, denn es geht von der freien Entfaltung der Bürger aus und akzeptiert Unterschiede im Erfolg, erkennt aber auch an, dass Unterschiede im Erfolg nicht nur auf Unterschieden in Leistung und Einsatzbereitschaft, sondern auch aus unterschiedlichen Ausgangsbedingungen resultieren können. ${ }^{199}$ Chancengleichheit verlangt zum einen nach Sicherstellung der rechtlichen Gleichheit der Chancen, damit nach Rechtsgleichheit, zum anderen nach Schaffung tatsächlicher Chancengleichheit als Voraussetzung individueller und gleicher Freiheitsausübung und ist damit weniger Forderung des Gleichheitssatzes als des sozialen Staatsziels und der Freiheitsrechte. ${ }^{200}$ Sozialstaatlich und freiheitsrechtlich gefordert sind gleiche reale Chancen zur freien Entfaltung der Persönlichkeit, etwa durch Bildungsförderung201, aber auch durch Präventionsmaßnahmen „zur Verminderung sozial bedingter Ungleichheit von Gesundheitschancen“202 als einen „Beitrag zu mehr Chancengleichheit bei der Gesundheit“"203.

${ }_{197}$ Vgl. Starck, in: v. Mangoldt/Klein/Starck (Fn. 196), Art. 3 Rn. 4, 35; s. auch: Di Fabio Soziale Gerechtigkeit (Fn. 15), 56f.; W. Schmitt Glaeser Der freiheitliche Staat des Grundgesetzes, 2008, 228; F. Schoch Der Gleichheitssatz, DVBl. 1988, 863 (880); K. Stern Das Staatsrecht der Bundesrepublik Deutschland, Bd. I, 2. Aufl. 1984, 930.

198 H. Maurer Staatsrecht I, 5. Aufl. 2007, § 6 Rn. 78.

199 Papier Zukunft des Sozialstaates (Fn. 51), 7.

${ }^{200} \mathrm{Zu}$ den verfassungsrechtlichen Grundlagen: Boysen Gleichheit im Bundesstaat (Fn. 62), $175 \mathrm{ff}$; Engels Chancengleichheit und Bundesstaatsprinzip (Fn. 62), 26ff.; Starck, in: v. Mangoldt/Klein/Starck (Fn. 196), Art. 3 Rn. 33; s. auch: Gröschner, in: Dreier (Fn. 21), Art. 20 (Sozialstaat) Rn. 40, zur Chancengleichheit als einem Aspekt sozialstaatlicher Gleichheit.

${ }^{201}$ Zur Bedeutung der Bildungsgleichheit als Voraussetzung für die Wahrnehmung der staatsbürgerlichen Rechte und der Freiheitsrechte: Kirchhof Gleichheitssatz (Fn. 76), § 124 Rn. 114ff. - Zur Bedeutung der Kindergartenerziehung: BVerfGE 97, 332 (347f.); zur Ausbildungsförderung als Maßnahme zur Herstellung von Chancengleichheit: I. Richter Ausbildungsförderung, in: Sozialrechtshandbuch (Fn. 112), § 21 Rn. 2 f., $63 \mathrm{ff}$.

$202 \S 20$ Abs. 1 S. 2 SGB V; zur Bedeutung der sozialen Gesundheitsfaktoren für den Gesundheitszustand: S. Huster Gesundheitsgerechtigkeit: Public Health im Sozialstaat, JZ 2008, 859 ff.

${ }^{203}$ BT-Drucks. 14/1977, 160. 
Doch selbst wenn die Verfassung - in den Worten Günther Dürigs „den Sockel der Ausgangschancen“ nach oben anzuheben hat ${ }^{204}$ oder wie es im Zweckartikel der Schweizerischen Bundesverfassung heißt ${ }^{205}$ die Eidgenossenschaft „für eine möglichst grosse Chancengleichheit“ zu sorgen hat, vermag der Staat gleiche Ausgangsbedingungen nicht allumfassend herzustellen, weil zu viele unterschiedliche Faktoren, die in einer freien Gesellschaft dem staatlichen Einfluss entzogen und Teil der Persönlichkeit sind, ${ }^{206}$ auf diese einwirken; Glück lässt sich nicht als Sozialleistung organisieren. ${ }^{207}$ Absolute, vollumfängliche Gleichheit in den Ausgangsbedingungen würde im Ergebnis wiederum zu strikter Egalität führen, die die liberale Verfassungssubstanz des freiheitlichen Sozialstaats und damit das Differenzierungspotential der Gesellschaft vernichten würde. ${ }^{208}$

Stößt die umfassende Herstellung von Chancengleichheit auf praktische wie verfassungsrechtliche Grenzen, so fordert der freiheitliche Sozialstaat allerdings, Chancengleichheit zumindest in dem Maße zu verwirklichen, dass die soziale Durchlässigkeit in der Gesellschaft gewährleistet und ein sozialer Aufstieg möglich ist. ${ }^{209}$ In der Aufstiegsfrage kommt gerade auch die Integration und Kohäsion des Sozialen zum Ausdruck. ${ }^{210}$ Ein Aufstieg ist allerdings nur dann möglich, wenn die Gesellschaft nach oben hin offen ist, so dass es nicht Sinn und Zweck sozialer Gleichheit sein kann, durch Deckelung und Nivellierung der Lebensverhältnisse nach unten, „Bewegung“ nach oben und Dynamik, die gerade wesentliche Elemente freiheitlicher Gesellschaften sind ${ }^{211}$, zu un-

204 Maunz/Dürig (Fn. 20), Art. 3 Abs. 1 Rn. 113, 140.

205 Art. 2 Abs. 3 BV. - Zu dieser Vorschrift: B. Ehrenzeller, in: Die schweizerische Bundesverfassung (Fn. 118), Art. 2 Rn. 1 ff. Danach sind dem Zweckartikel weder Handlungskompetenzen oder Gesetzgebungsaufträge noch verfassungsmäßige Rechte zu entnehmen, doch ist die Zweckbestimmung als rechtlich verbindliche Handlungsrichtlinie zu beachten; sie dient auf jeden Fall als Auslegungshilfe und ist bei der Konkretisierung offener Verfassungsnormen in Rechtsetzung und Rechtsanwendung heranzuziehen (ebd., Rn. 11).

206 Starck, in: v. Mangoldt/Klein/Starck (Fn. 196), Art. 3 Rn. 33.

207 Vgl. Bundespräsident Köhler Zur Freiheit gehört Ungleichheit (Fn. 84), 3.

208 Rüfner, in: Bonner Kommentar (Fn. 54), Art. 3 Abs. 1 Rn. 58; vgl. dazu auch: Gröschner, in: Dreier (Fn. 21), Art. 20 (Sozialstaat) Rn. 40; R. Stettner Der Gleichheitssatz, BayVB1. 1988, 545 (552). - Deutlich auf die Gefahr einer extensiv verwirklichten Chancengleichheit hinweisend: W. Leisner Chancengleichheit als Form der Nivellierung, FS Klecatsky, 1. Teilbd., 1980, $535 \mathrm{ff}$.

209 Vgl. Rüfner, in: Bonner Kommentar (Fn. 54), Art. 3 Abs. 1 Rn. 63, der insoweit von einer Pflicht des demokratischen Sozialstaats spricht.

210 Vogel Prekarität und Prekariat (Fn. 29), 18.

211 Zur „Bewegung“ als wesentliches Element freiheitlicher Gesellschaften Pitschas Frei - sozial - auch sicher? (Fn. 15), 297. 
terbinden. Soziale Ungleichheiten sind insoweit auch notwendig und förderlich, wenn und solange sie noch eine dynamische Kraft besitzen, von ihr motivierende Impulse ausgehen, sie durch eigenes Engagement überwunden werden können und schöpferische Kräfte freisetzen. Allerdings scheint hierin zur Zeit gerade ein großes Problem zu liegen, wenn nicht einmal die Hälfte der Bevölkerung glaubt, dass sich Leistung lohne und mit sozialem Aufstieg belohnt werde, sich stattdessen Statusfatalismus breit macht ${ }^{212}$ und sich die Zone sozialer Verwundbarkeit angesichts zunehmender Prekarisierung ausweitet. ${ }^{213}$

\section{Soziale Gleichheit und Rechtsgleichheit}

Jenseits spezieller grundgesetzlicher Vorschriften, etwa der Gleichstellung nichtehelicher Kinder 214 oder der besonderen Gleichheitssätze ${ }^{215}$, die vor Benachteiligung aufgrund bestimmter, oftmals gerade auch soziale Ungleichheit begründender Eigenschaften schützen und zudem - wie insbesondere das Verbot der Benachteiligung Behinderter zeigt $^{216}$ - auch konkrete Impulse und Aufträge zur Kompensation sozialer Nachteile geben können, ${ }^{217}$ lassen sich gesetzliche Maßnahmen zur Herstellung sozialer Gleichheit vor dem allgemeinen Gleichheitssatz aus dem sozialen Staatsziel rechtfertigen. Obwohl auf Rechtsgleichheit zielend, kann und darf der allgemeine Gleichheitssatz soziale Ungleichheit nicht unberücksichtigt lassen, ${ }^{218}$ sondern muss diese aufnehmen und

${ }^{212}$ S. dazu Heuser Ungleichheit ist nicht immer schlecht (Fn. 13), 23. - Nach Auffassung von H. Krüger (Allgemeine Staatslehre, 2. Aufl. 1966, 811) kann ein Staat, in dem es nicht möglich ist durch echte Leistung aufzusteigen, in einer Zeit, in der Aufstieg ein soziales Ideal ist, nicht mehr bestehen.

213 Vogel Staatsbedürftigkeit der Gesellschaft (Fn. 11), $78 \mathrm{ff}$.

214 Art. 6 Abs. 5 GG. - S. dazu: P. Badura, in: Maunz/Dürig (Fn. 20), Art. 6 Rn. 175 ff.; G. Robbers, in: v. Mangoldt/Klein/Starck (Fn. 196), Art. 6 Rn. 305 ff.; Rüfner Leistungsrechte (Fn. 95), § 40 Rn. 25.

${ }^{215} \mathrm{Zu}$ dem Problem, ob und in welcher Weise die unterschiedlichen besonderen Gleichheitssätze im Einzelnen als Gebot sozialer Gleichheit wirken: F. Welti Behinderung und Rehabilitation im sozialen Rechtsstaat, 2005, $454 \mathrm{ff}$.

${ }^{216}$ Dazu grundlegend und umfassend: Welti Behinderung und Rehabilitation (Fn. 215); s. auch: U. Davy Das Verbot der Diskriminierung wegen einer Behinderung im deutschen Verfassungsrecht und im Gemeinschaftsrecht, SDSRV 49 (2002), 7 ff.; Pitschas Sicherungssysteme (Fn. 46), 840.

217 Vgl. Zacher Staatsziel (Fn. 21), § 28 Rn. 37. - Nach BVerfGE 113, 1 (15) bietet Art. 3 Abs. 2 GG im Hinblick auf die Gleichberechtigung der Geschlechter auch Schutz vor faktischen Benachteiligungen und zielt auf die Angleichung der Lebensverhältnisse.

218 Zacher Staatsziel (Fn. 21), § 28 Rn. 37. - Zur historischen Entwicklung des Verhältnisses von Rechtsgleichheit und sozialer Gleichheit: R. Zippelius Der Gleichheitssatz, VVDStRL 47 (1989), 7 (13 ff.). 
verarbeiten. Rechtliche und soziale Gleichheit ${ }^{219}$ stehen nicht in einem scharfen Gegensatz ${ }^{220}$ und beziehungslos nebeneinander, ${ }^{221}$ denn soziale Gleichheit bildet bereits eine Voraussetzung rechtlicher Gleichheit und dient dieser.

Im Rahmen des allgemeinen Gleichheitssatzes lässt sich zudem die Frage, ob eine nicht zu rechtfertigende rechtliche Ungleich- oder Gleichbehandlung vorliegt, nicht losgelöst von den realen Auswirkungen und tatsächlichen Folgen der Norm in der von ihr geprägten sozialen Wirklichkeit beantworten.222 Der Gesetzgeber hat die Realität wirklichkeitsgetreu aufzunehmen, die tatsächlichen Ungleichheiten des zu ordnenden Lebenssachverhalts zu berücksichtigen ${ }^{223}$ und den Regelungsgegenstand realitätsgerecht $\mathrm{zu}$ bewerten. ${ }^{24}$ Gleichheitsgerechtes Recht ist realitätsverpflichtet. ${ }^{225}$

${ }^{219} \mathrm{Im}$ Kontext des Art. 3 GG wird oftmals auch von realer, faktischer oder materieller Gleichheit gesprochen; zur unterschiedlichen Terminologie: Welti Behinderung und Rehabilitation (Fn. 215), 430. - Kritisch zum Begriff der faktischen Gleichheit: C. Starck Diskussionsbeitrag, VVDStRL 47 (1989), 78 (80).

220 Eine Trennung dagegen befürwortend: Starck, in: v. Mangoldt/Klein/Starck (Fn. 196), Art. 3 Rn. 4 ff., der faktische Gleichheit als Kontrastbegriff bezeichnet (aaO, Rn. 4); ders. Die Anwendung des Gleichheitssatzes, in: C. Link (Hrsg.) Der Gleichheitssatz im modernen Verfassungsstaat, 1982, 51 (55ff., $67 \mathrm{ff}$.); anders dagegen etwa: R. Alexy Theorie der Grundrechte, 1985, $380 \mathrm{ff}$.; M. Borowski Grundrechte als Prinzipien, 2. Aufl. 2007, 395 ff.; Hesse Gleichheitssatz im Staatsrecht (Fn. 81), 178 ff., 207 ff.; Heun, in: Dreier (Fn. 21), Art. 3 Rn. 66; ders. Gleichheit (Fn. 121), § 34 Rn. 50; Osterloh, in: Sachs (Fn. 117), Art. 3 Rn. 44; Welti Behinderung und Rehabilitation (Fn. 215), 430 ff.; Zippelius Gleichheitssatz (Fn. 218), 15 ff. - Zu Recht weist G. Müller Der Gleichheitssatz, VVDStRL 47 (1989), 37 (54), darauf hin, dass es im Ergebnis nicht so wesentlich zu sein scheint, ob ein Egalisierungsgebot als Ausfluss des Gleichheitssatzes oder des Sozialstaatsprinzips oder einer Verbindung beider Normen betrachtet wird. Nach Ansicht von W. Rüfner Der Gleichheitssatz im Sozialrecht und die Aufgabe der Verfassungsrechtsprechung, SGb 1984, 147 (148, Fn. 31), handelt es sich nur um eine Frage der Konstruktion, nicht des Ergebnisses.

221 Haller Verrechnung von Vor- und Nachteilen (Fn. 121), 293 f.; S. Huster Rechte und Ziele, 1993, 408 ff.; G. Robbers Der Gleichheitssatz, DÖV 1988, 749 (757); Rüfner Gleichheitssatz im Sozialrecht (Fn. 220), 148. - Nach Schoch Gleichheitssatz (Fn. 197), 869 , sind Gleichheitssatz und Sozialstaatsprinzip einander verhältnismäßig zugeordnet.

222 Vgl. dazu: U. Kischel in: V. Epping/C. Hillgruber (Hrsg.) Beck'scher Online Kommentar zum Grundgesetz, Stand des Gesamtwerks: 2008, Art. 3 Rn. 87. Huster Rechte und Ziele (Fn. 221), 409 ff., weist zu Recht darauf hin, dass bereits der Vergleichsmaßstab von Wertungen bestimmt wird, die in den tatsächlichen Verhältnissen gründen.

$223 \mathrm{Vgl}$. BVerfGE 118, 1 (27).

${ }^{224}$ P. Kirchhof Grundrechtsinhalte und Grundrechtsvoraussetzungen, in: Handbuch der Grundrechte, Bd. I (Fn. 86), § 21 Rn. 22.

225 Kirchhof Gleichheitssatz (Fn. 76), § 124 Rn. 215. 
Doch selbst in Verbindung mit dem allgemeinen Gleichheitssatz ist das soziale Staatsziel kein andere Verfassungsbestimmungen überspielendes Egalisierungsgebot, um Wohlstandsdifferenzen zu beseitigen.226 Rechtliche Ungleichbehandlungen zum Zwecke des sozialen Ausgleichs sind vor Art. 3 Abs. 1 GG rechtfertigungsbedürftig, ${ }^{227}$ allerdings auch rechtfertigungsfähig. ${ }^{228}$

Besondere Bedeutung bei der verfassungsrechtlichen Beurteilung von Maßnahmen zur Herstellung sozialer Gleichheit kommt ihren Kosten $\mathrm{zu}$, beruht doch das sozialstaatliche Geben regelmäßig auf einem Nehmen durch Steuern ${ }^{229}$ oder Sozialversicherungsbeiträge. ${ }^{230}$ Staatliches Nehmen muss sich am rechtsstaatlichen und grundrechtlichen Schutz der bestehenden Güterzuordnung messen lassen sowie mit den finanzverfassungsrechtlichen Vorgaben und den gleichheitsrechtlichen Anforderungen der Verfassung, insbesondere dem Gebot der Belastungsgleichheit ${ }^{231}$, vereinbar sein. So hat etwa der Sozialversicherungsbeitrag als nichtsteuerliche Abgabe strukturelle Distanz zur Steuer zu halten und muss sich in Tatbestand und Legitimation von ihr unterscheiden. ${ }^{232}$ Seine Erhebung bedarf der Rechtfertigung aus dem Gedanken der sozialen Schutzbedürftigkeit des einbezogenen Personenkreises, der fehlenden Möglichkeit zu ausreichender eigenverantwortlicher Vorsorge

${ }^{226}$ Rüfner Gleichheitssatz im Sozialrecht (Fn. 220) 149. - Nach BVerfGE 12, 354 (367) ermächtigt das Sozialstaatsprinzip nicht zu beliebiger Sozialgestaltung, die das Gebot der Gleichheit auflösen würde.

${ }^{227}$ BVerfGE 99, 367 (395). Maßstab ist dabei in der Regel weniger das reine Willkürverbot, sondern das Verhältnismäßigkeitsprinzip auf der Grundlage der neuen Formel, weil zumeist Personen bzw. Personengruppen verschieden behandelt werden; zu den verfassungsgerichtlichen Rechtfertigungsmaßstäben bei der Gleichheitsprüfung vgl. nur mwN BVerfG, NJW 2008, 2409 (2417f.). Bei der Rechtfertigung sind etwa die Schwere und Folgen sozialer Ungleichheit für den Einzelnen und die Gesellschaft, die Möglichkeit zur eigenverantwortlichen Überwindung und der Grad der Beeinträchtigung rechtlicher Gleichheit zu berücksichtigen.

${ }^{228}$ Heun Gleichheit (Fn. 121), § 34 Rn. 50; Rüfner, in: Bonner Kommentar (Fn. 54), Art. 3 Abs. 1 Rn. 56, spricht davon, dass derjenige, der die rechtliche Gleichheit durchbrechen möchte, dafür einen zureichenden Grund haben müsse und ihn insoweit die Argumentationslast treffe.

${ }^{229}$ Zur Bedeutung des Steuerrechts für den sozialen Ausgleich deutlich das Sondervotum von E.-W. Böckenförde zum Vermögensteuerbeschluss (BVerfGE 93, 149 (163)); s. auch: Bryde Steuerverweigerung und Sozialstaat (Fn. 54), $326 \mathrm{ff}$.

230 S. dazu mwN Heinig Sozialstaat (Fn. 18), $555 \mathrm{ff}$.

${ }^{231}$ Zum Gebot der Belastungsgleichheit vgl. nur mwN: Kirchhof Steuern (Fn. 34), $\S 118$ Rn. $168 \mathrm{ff}$; speziell im Hinblick auf soziale Sicherungssysteme: Pitschas Sicherungssysteme (Fn. 46), 829, $842 \mathrm{f}$.

232 Dazu J. Isensee Finanzverfassung und Sozialrecht, SDSRV 35 (1992), 7 (17ff.). 
sowie einem angemessenen Verhältnis ${ }^{233} \mathrm{zu}$ den jeweiligen Leistungen. ${ }^{234}$ Verbesserung der Einnahmen allein oder als der wesentliche Grund rechtfertigen dagegen nicht schon die Einbeziehung weiterer Personen, allerdings hat sich das Bundesverfassungsgericht bislang unter Hinweis auf die finanzielle Stabilität der Sozialversicherung und den weiten sozialpolitischen Gestaltungsspielraum des Gesetzgebers insoweit eher großzügig gezeigt. ${ }^{235}$

\section{Soziale Gleichheit und demokratischer Prozess}

Das Grundgesetz sieht zwar in der Herstellung sozialer Gleichheit im Rahmen der grundrechtlichen und rechtsstaatlichen Vorgaben ein Ziel staatlichen Handelns, doch schweigt es über das exakte Maß der konkret in der jeweiligen Situation anzustrebenden Gleichheit ${ }^{236}$ und den genauen Weg, auf dem das Ziel zu erreichen ist. ${ }^{237}$ Da zudem Inhalt und Umfang sozialer Bedürfnisse zeit- und situationsabhängig sind, ihre Erfüllung dem „Vorbehalt des Möglichen“238 unterliegt und sich die Leistungskapazität des Gemeinwesens umfassender grundgesetzlicher Regelung entzieht, würde es die Verfassung überfordern, wenn sie vollzugsfertige Ansprüche auf soziale Leistungen zur Herstellung sozialer Gleichheit gewähren würde ${ }^{239}$ und das Maß sozialer Gleichheit exakt festschriebe. Über das rechte Maß sozialer Gleichheit in der jeweiligen Situation und die dafür konkret erforderlichen Maßnahmen hat im sozialen Rechtsstaat des Grundgesetzes der Gesetzgeber im demokratischen Prozess zu entscheiden. ${ }^{240}$ Dabei kommt ihm aufgrund der Weite

${ }^{233}$ Zum Zusammenhang von Zwangsversicherung mit Beitragspflicht und Leistungsumfang deutlich BVerfGE 115, 25 (42ff.).

234 Zum Freiheitsschutz gegenüber der Erhebung von Sozialversicherungsbeiträgen jüngst Heinig Sozialstaat (Fn. 18), 565 ff. mwN.

235 Vgl. nur: BVerfG, NZS 2005, 479 ff. - Kritisch dazu: H. Bethge/C. von Coelln Die gesetzliche Erhöhung der Versicherungspflichtgrenze in der Gesetzlichen Krankenversicherung als möglicher Verstoß gegen Grundrechte privater Versicherungsunternehmen, VSSR 2004, 199 ff.; allgemein: F. Hufen Grundrechtsschutz der Leistungserbringer und privaten Versicherer in Zeiten der Gesundheitsreform, NJW 2004, 14 (16ff.).

236 Rüfner Gleichheitssatz im Sozialrecht (Fn. 220), 149.

237 BVerfGE 59, 231 (263). - S. dazu auch: Papier Grundrechte und Sozialordnung (Fn. 100), § 30 Rn. 8.

238 Zum „Vorbehalt des Möglichen“: BVerfGE 33, 302 (333ff.); 82, 60 (82); Heinig Sozialstaat (Fn. 18), 383 ff.; P. Kirchhof Mittel staatlichen Handelns, HStR V, 3. Aufl. 2007, § 99 Rn. $108 \mathrm{ff}$.

239 Vgl. Isensee Grundrechtsvoraussetzungen (Fn. 68), § 115 Rn. 149.

240 Zur Harmonie und zu den Spannungen zwischen „sozial“ und „Demokratie“: Pitschas Sicherungssysteme (Fn. 46), 834f.; Zacher Staatsziel (Fn. 21), § 28 Rn. 101 ff.; 
und Unbestimmtheit der sozialstaatlichen Ermächtigung ${ }^{241}$ ein weiter Spielraum zu, ${ }^{242}$ so dass es ihm etwa auch möglich ist, soziale Leistungen zu kürzen, da das Grundgesetz kein generelles soziales Rückschrittsverbot kennt. ${ }^{243}$ Doch wird die demokratische, auf Wiederwahl hoffende Mehrheitsherrschaft schon aufgrund der tatsächlichen Eigengesetzlichkeiten und der oftmals auch wirkmächtigen und öffentlichkeitswirksam erhobenen Forderungen tatsächlich oder vermeintlich Benachteiligter durch ein Mehr an Leistungen hin zu einer weiteren Angleichung der realen Lebensverhältnisse und zu mehr Chancengleichheit für alle zur Teilhabe an höherem Wohlstand tendieren, ${ }^{244} \mathrm{da}-$ mit nach einem Mehr an sozialer Gleichheit ${ }^{245}$ streben.

\section{Soziale Gleichheit als Thema der Verfassung}

Soziale Gleichheit, die auf umfassende Egalisierung, Standardisierung und Konfektionierung zielt, ist eine Utopie, die weder Voraussetzung noch Aufgabe der Verfassung ist und als solche auch nicht erodieren konnte. Wird soziale Gleichheit dagegen auf die Angleichung und Hebung der Lebensverhältnisse durch Schaffung von Chancengleichheit im Sinne der Herstellung gleicher Startbedingungen zur Teilhabe aller an mehr Wohlstand bezogen, so ist sie sowohl Aufgabe als auch Voraussetzung des freiheitlichen Sozialstaates.

allgemein zum Verhältnis von Demokratie und Sozialstaat s. auch: Böckenförde Demokratie (Fn. 75), § 24 Rn. 97 ff.

${ }^{241}$ Vgl. BVerfGE 94, 214 (263); Butzer Sozialstaatsentwicklung (Fn. 15), 57 ff.; Haller Verrechnung von Vor- und Nachteilen (Fn. 121), 291; Neumann Sozialstaatsprinzip (Fn. 122), 92.

${ }^{242}$ BVerfGE 97, 169 (185); 103, 271 (288); sehr deutlich und weitgehend BVerfGE 103, 293 (307): „Auf dem Gebiet der Arbeitsmarkt-, Sozial- und Wirtschaftsordnung gebührt dem Gesetzgeber ein besonders weitgehender Einschätzungs- und Prognosevorrang".

${ }^{243}$ Isensee Grundrechtsvoraussetzungen (Fn. 68), § 115 Rn. 161; ders. Verwaltung des Mangels im Gesundheitswesen - verfassungsrechtliche Maßstäbe der Kontingentierung, GS Heinze, 2005, 417 (431); Neumann Sozialstaatsprinzip (Fn. 122), 97f.; zur Frage eines sozialen Rückschrittsverbots umfassend: R.-U. Schlenker Soziales Rückschrittsverbot und Grundgesetz, 1986.

${ }^{244}$ S. dazu: Rüfner, in: Bonner Kommentar (Fn. 54), Art. 3 Abs. 1 Rn. 57; Zippelius Gleichheitssatz (Fn. 218), $15 \mathrm{f}$.

${ }^{245}$ Grundlegend zu dem Ziel und dem gemeinsamen Nenner aller Erscheinungsformen des Sozialen „Mehr Gleichheit“: Zacher Sozialstaat (Fn. 21), § 28 Rn. 39 ff., 53 ff., $86 \mathrm{ff}$; ders. Der europäische Sozialstaat (Fn. 35), 4. 
Leitsätze des 2. Berichterstatters über:

\section{Soziale Gleichheit - Voraussetzung oder Aufgabe der Verfassung?}

\section{Soziale Ungleichheit als Problem}

1) Soziale Ungleichheit wird in Deutschland in immer stärkerem Maße wahrgenommen und von weiten Teilen der Bevölkerung als nicht mehr hinnehmbar empfunden. Vor diesem Hintergrund besteht gerade in einer Gesellschaft wie der deutschen, in der der Sozialstaat und der Gedanke der sozialen Gerechtigkeit tief verwurzelt sind, die Gefahr dauerhafter, den sozialen Frieden bedrohender Spannungen und Spaltung.

2) Die Forderungen nach Abbau und Beseitigung sozialer Ungleichheit zielen auf die Herstellung und Hebung sozialer Normalität.

3) Adressat der Forderungen ist noch immer in erster Linie der Nationalstaat, weil dieser, ungeachtet der Globalisierung und der Bedeutung der europäischen Integration für das Soziale, die wichtigste Ebene für die Gewährleistung von sozialer Sicherheit und sozialem Ausgleich ist.

4) Bezog sich die soziale Frage des 19. Jahrhunderts insbesondere auf die Arbeiterschaft als benachteiligte Gruppe, so wurden in der Folgezeit immer neue Konstellationen individueller oder gruppenhafter sozialer Schlechterstellungen wahrgenommen. Heute gelten insbesondere Prekarität und Prekariat als Signalwörter neuer sozialer Ungleichheit.

5) Selbst wenn fundamentale Ungleichheitsrelationen die Peripheren in den Hintergrund treten lassen, öffnet der Ausgleich einer anerkannten sozialen Ungleichheit den Blick auf neue Ungleichheiten, die ihrerseits nach Kompensation rufen. Der Horizont sozialer Gleichheit ist in stetiger Bewegung.

6) Obwohl der Begriff des „Sozialen“ weit und offen ist, gelten vor allem solche Ungleichheiten als problematisch, die ihre Ursache oder ihre Wirkung in ökonomischen Ungleichheiten haben. Selbst wenn nichtökonomische Interventionen für das "Soziale“ ebenso relevant wie notwendig sind, konzentrieren sich die Forderungen nach sozialer Gleichheit auf ökonomisch wirkende oder relevante Maßnahmen zur Verbesserung der Lebensverhältnisse. 


\section{Soziale Gleichheit als Aufgabe von Staat und Gesellschaft}

7) In der freiheitlichen demokratischen Wirtschafts- und Sozialordnung des Grundgesetzes sind die Angleichung und Verbesserung der Lebensverhältnisse weder alleinige noch primäre Aufgaben des Staates. Das grundgesetzliche Sozialstaatsprinzip normiert zwar ein Staatsziel, jedoch keinen Staatsvorbehalt für das Soziale. Der Abbau sozialer Ungleichheit ist zwar eine Staatsaufgabe, doch ist diese dem Staat nur im Sinne einer Letzt- und Gesamtverantwortung zugewiesen.

\section{Sinn und Zweck sozialer Gleichheit}

8) Selbst wenn im demokratischen und sozialen Bundesstaat die unitarisierend wirkende Forderung nach sozialer Gleichheit föderal gebrochen ist, weil er Vielfalt und Unterschiede vorsieht und fordert, damit auch regionale soziale Ungleichheit zulässt, ist er um seiner Stabilität willen auf ein Mindestmaß an sozialer Gleichheit angewiesen und hat darauf zu achten, dass die sozialen Unterschiede im Bundesgebiet nicht stetig wachsen und das bundesstaatliche Sozialgefüge beeinträchtigen.

9) Gleiche rechtliche Freiheit erzeugt soziale Ungleichheit und ist deren permanente Quelle, denn die liberalen Freiheitsrechte des Grundgesetzes bieten jedermann die gleiche rechtliche Freiheit an ohne Ansehen seiner Person, seiner Bedürfnisse, seiner Fähigkeiten oder Verdienste.

10) Der Staat hat die Aufgabe, die realen Voraussetzungen der Grundrechtsausübung zu sichern, den Freiheitsrechten damit gesellschaftliche Effektivität und Breitenwirkung zu geben und so dem Einzelnen ein selbstbestimmtes Leben in der Gesellschaft zu ermöglichen.

\section{Inhalt und Umfang sozialer Gleichheit}

11) Das Rechtsstaatsprinzip leitet und begrenzt nicht nur staatliche Maßnahmen zur Herstellung sozialer Gleichheit, dadurch dass diese im Verfahren und in den Formen des Rechtsstaats erfolgen müssen, sondern es bildet auch den Grund, auf dem sich soziale Gleichheit erst entfalten kann und steht schon deshalb nicht dem sozialen Staatsziel unvereinbar gegenüber.

12) Das soziale Staatsziel bezieht sich auf Freiheitsvoraussetzungen, indem es auf die ungleichen tatsächlichen Lebensverhältnisse und die realen Bedürfnisse und Fähigkeiten des Einzelnen blickt. Der grundrechtlichen Freiheit soll ein Mindestmaß an sozialer Sicherheit und der grundrechtlichen Gleichheit ein Mindestmaß an sozialer Gleichheit korrespondieren. 
13) Originäre Ansprüche auf bestimmte Maßnahmen zur Herstellung sozialer Gleichheit lassen sich grundsätzlich nicht aus dem sozialen Staatsziel, selbst in Verbindung mit dem allgemeinen Gleichheitssatz, begründen. Doch können sich im Einzelfall von den jeweiligen Grundrechten her Ansprüche auf eine Mindestsicherung ergeben, etwa auf die Gewährleistung des Existenzminimums.

14) Ist die Forderung nach sozialer Gleichheit regelmäßig mit dem Wunsch nach sozialpolitischer Aktivität verbunden, so zielt sie gerade auf dem Feld der sozialen Sicherheit auch auf Stetigkeit, Verlässlichkeit, Berechenbarkeit, auf ein in sich stimmiges und transparentes Konzept. Die Hektik ständiger Änderungen, die Pluralität und Komplexität sozialrechtlicher Leistungen sowie die unterschiedlichen Zuständigkeiten im Sozialen überfordern die Betroffenen und erzeugen Unübersichtlichkeit.

15) Im freiheitlichen Sozialstaat des Grundgesetzes zielt die Forderung nach sozialer Gleich heit auf die Herstellung von Chancengleichheit im Sinne der Gewährleistung gleicher Startbedingungen und gleicher Entwicklungschancen zur Teilhabe am wirtschaftichen und gesellschaftichen Leben, nicht dagegen auf Ergebnisgleichheit.

16) Sozialstaatliches Geben zur Herstellung sozialer Gleichheit beruht regelmäßig auf einem Nehmen durch Steuer oder Sozialversicherungsbeitrag, das wiederum dem rechtsstaatlichen und freiheitsrechtlichen Schutz der bestehenden Güterzuordnung und den gleichheitsrechtlichen Vorgaben der Verfassung genügen muss.

17) Das Grundgesetz sieht zwar in der Herstellung sozialer Gleichheit im Rahmen der grundrechtlichen und rechtsstaatlichen Vorgaben ein Ziel staatlichen Handelns, doch schweigt es über das exakte Maß der konkret in der jeweiligen Situation anzustrebenden sozialen Gleichheit und den genauen Weg, auf dem das Ziel zu erreichen ist. Darüber ist im demokratischen Prozess zu entscheiden.

\section{Soziale Gleichheit als Thema der Verfassung}

18) Soziale Gleichheit im Sinne umfassender und völliger Egalität ist eine Utopie, die weder Voraussetzung noch Aufgabe der Verfassung ist. Wird soziale Gleichheit dagegen auf die Angleichung und Hebung der Lebensverhältnisse durch Schaffung von Chancengleichheit im Sinne gleicher Startbedingungen zur Teilhabe aller an mehr Wohlstand bezogen, so ist sie sowohl Voraussetzung als auch Aufgabe des freiheitlichen Sozialstaats des Grundgesetzes. 


\section{Aussprache und Schlussworte}

\section{Soziale Gleichheit - Voraussetzung oder Aufgabe der Verfassung?}

Morlok: Soziale Gleichheit - ein großes Wort, wer's denn recht verstünde. Ich glaube, man kann das Thema in mehreren Aspekten behandeln - mehrere Aspekte, die auch immer als Gegensatzpaare dargestellt werden können. Also etwa Freiheit und Gleichheit; oder rechtliche Freiheit auf der einen Seite, tatsächliche Gleichheit auf der anderen Seite; oder ein drittes Gegensatzpaar: Chancengleichheit und Ergebnisgleichheit. Beide Referenten haben sich mit diesen Aspekten lebhaft auseinander gesetzt und ich glaube, ich muss das hier jetzt nicht wiederholen. Klar ist, um zum ersten zurückzukommen, Ungleichheit ist eben auch die Frucht von Gleichheit, oder umgekehrt: Gleichheit wird nur ermöglicht durch Einschränkung der Freiheit. Lassen Sie mich aber diesen beiden Gegensätzen noch zwei weitere hinzufügen.

Der eine ist folgender: Mitleid kommt immer zu spät, Kompensation bringt meistens zu wenig, soziale Ungleichheit ist ein Produkt des gesellschaftlichen Prozesses. Sehr viel effektiver, als hinterher Salbe aufzustreichen, ist es, die Produktion sozialer Ungleichheit zu unterbinden, wenn man nach sozialer Gleichheit strebt. Das klang im Referat an, in beiden Referaten, wenn ich das recht im Ohr habe, etwa im Hinblick auf die Bildungspolitik. Ich sehe eben Herrn Huster vor mir sitzen, er hat gerade in einem schönen JZ-Aufsatz herausgearbeitet, dass die Behandlung von Krankheiten eben im Zweifelsfall zu spät kommt. Die Prävention ist wichtiger. Dass ich eben dafür sorge, dass Leute nicht an Lärmschäden leiden, sondern dass rechtzeitig Lärmschutz gemacht wird.

Für unser Thema bedeutet das die Frage - und ich fand sie in den Referaten ein bisschen unterbelichtet -: Was ist dem Gesetzgeber erlaubt an Freiheitseinschränkungen unter dem Ziel der Beförderung der sozialen Gleichheit? Im zweiten Referat bei Herrn Axer tauchte das sehr wohl auf, aber immer nicht als direktes Ziel des Gesetzgebers, sondern immer nur in Gestalt der Schrankendiskussion bei den Freiheitsrechten. Jetzt würde mich interessieren, den Akzent auf die andere Seite zu setzen: Was ist zur Erreichung dieses Ziels zulässig?

Der zweite Aspekt betrifft die Frage, welcher Faktor befördert unter den Bedingungen unserer Demokratie die soziale Gleichheit - sofern sie 
mit rechtlichen Mitteln herstellbar ist - am stärksten? Das ist das allgemeine Wahlrecht. Das kam in den Referaten leider nicht vor. Dabei um den alten Marx zu variieren: Das Wahlrecht, das gleiche Wahlrecht, das ist der große Leveller, also der Gleichmacher. Auch wenn wir im Referat das Homogenitäts-Theorem gehört haben, dass die Demokratie ein Mindestmaß an sozialer Homogenität braucht, so könnte man ja fast von der List des demokratischen Sozialstaats sprechen, dass eben die Demokratie mit ihrem gleichen Wahlrecht und den ihnen eingebauten Mechanismen dafür sorgt, dass die sozialen Diskrepanzen nicht zu groß werden. Dankeschön.

Meyer: Das Thema lautet „Soziale Gleichheit: Voraussetzung oder Aufgabe der Verfassung?“. Wenn beide Referenten es ernst genommen hätten, hätten sie mit einem „Nein“ auf die Frage ihre Referate beenden können. Tatsächlich haben sie auch nicht behauptet, dass soziale Gleichheit Voraussetzung oder Aufgabe des Staates ist. Die unsichere Fragestellung hat aber dazu geführt, dass es zu unsauberen Argumentationen gekommen ist. Ich will das an dem Beispiel von Herrn Axer darlegen. Wenn Sie dessen These 7 nehmen, dann steht dort, dass die Angleichung der Lebensverhältnisse zwar weder alleinige noch primäre, aber eben doch Aufgabe des Staates ist, während er zwei Sätze später sehr viel richtiger sagt, der Abbau sozialer Ungleichheit gehöre zu den Aufgaben des Staates. Das sind aber zwei Paar Schuhe. Wenn Sie, Herr Axer, in These 8 sagen, dass der Staat um seiner Stabilität willen darauf $\mathrm{zu}$ achten habe, dass die sozialen Unterschiede im Bundesgebiet nicht stetig wachsen, so wird er das nicht machen können. Denn jede Wohlstandserhöhung führt automatisch zu Unterschieden, vor allen Dingen zu regionalen Unterschieden, und ich wüsste nicht, woraus Sie schließen könnten, dass der Staat von Verfassungs wegen gezwungen wäre, diese auszugleichen, und er tut es auch nicht. In der These 10 steht, der Staat habe die Aufgabe, die realen Voraussetzungen der Grundrechtsausübung zu sichern. Wenn Sie das ernst nehmen, dann würde ich als Banker eine Privatbank aufmachen und dem Staat sagen: Nun gib mal die Grundfinanzierung für eine solche Bankeröffnung. Das sind große Worte, die praktisch nicht realisiert werden können und auch nicht realisiert werden. In These 12 sagen Sie, dass der grundrechtlichen Freiheit ein Mindestmaß an sozialer Sicherheit und der grundrechtlichen Gleichheit ein Mindestmaß an sozialer Gleichheit korrespondieren solle. Wenn das ernsthaft gemeint wäre, würde der Staat völlig überfordert sein, und deshalb denke ich, es ist nicht unsere Aufgabe, dem Staat eine Aufgabe zuzuweisen, die er nicht erfüllen kann. In der These 18 sagen Sie „Wird soziale Gleichheit dagegen auf die Angleichung und Heben der Lebens- 
verhältnisse zur Schaffung von Chancengleichheit im Sinne gleicher Startbedingungen ... bezogen“. Es ist ja ganz richtig, dass der Staat das macht, aber das führt automatisch zur Schaffung von sozialer Ungleichheit. Gleiche Startbedingungen führen, weil die Menschen eben ungleich sind, ungleich in ihrer Arbeit und in deren Auswirkungen, zur sozialen Ungleichheit. In Wirklichkeit kommen Sie also dazu zu sagen, das Thema ist falsch gestellt und worum es eigentlich nur geht, ist, wie viel Ungleichheit der Staat tolerieren darf oder sollte oder wann und wozu er gezwungen ist einzugreifen, wenn die Ungleichheit zu stark wird. Alles andere ist verfassungsrechtliche Poesie.

Nußberger: Unser Ausgangspunkt ist doch, dass der Begriff „soziale Gleichheit" nicht im Grundgesetz steht. Auf der einen Seite haben wir den Gleichheitssatz und auf der anderen Seite das Sozialstaatsprinzip, aber den Grundsatz der sozialen Gleichheit gibt es gerade nicht. Deshalb ist meine Frage an die beiden Referenten: Worum geht es eigentlich - um Verfassungsrecht oder um Verfassungspolitik? Und wie gehen wir dogmatisch vor? Nehmen wir den Gleichheitssatz, argumentieren, Gleiches müsse gleich, Ungleiches ungleich behandelt werden und integrieren die soziale Dimension in die Interpretation von Art. 3 GG, indem wir den Gleichheitssatz gewissermaßen sozial anreichern? Oder nehmen wir die soziale Dimension ganz aus dem Gleichheitssatz heraus und stützen uns auf das Sozialstaatsprinzip? Diese Frage würde ich gerne beantwortet haben. Die Frage nach einer Abgrenzung zwischen Verfassungsrecht und Verfassungspolitik richtet sich auch konkret an Sie, Frau Davy, und ist auch auf Ihre dritte Ebene, die internationale Ebene, bezogen. Sprechen Sie in diesem Zusammenhang von Völkerrecht und aus dem Völkerrecht ableitbaren Forderungen - etwa beim „benchmarking“ - oder geht es auch hier letztlich um Völkerrechtspolitik?

Ebsen: Zunächst eine Vorbemerkung: Die Komplexität der Frage, ob soziale Gleichheit Voraussetzung oder Aufgabe der Verfassung sei, zeigt sich - insofern habe ich die Referenten etwas anders verstanden als Herr Meyer - in der deutlichen, wenn auch vordergründig gegensätzlichen Weise ihrer Antworten (Frau Davy letztlich: weder noch; Herr Axer letztlich: sowohl als auch), wobei ich beiden zustimmen kann. Es kommt eben darauf an, was man unter sozialer Gleichheit, Verfassungsvoraussetzung und Verfassungsaufgabe versteht.

Beim Thema der Verfassungsvoraussetzung geht es in der Tat um das von Herrn Axer angesprochene Mindestmaß an sozialer Homogenität. Dies hat allerdings mehr Facetten als diejenige der sozialen Gleichheit/ 
Ungleichheit im Sinne eines Unten und Oben, also der Schichtendifferenzierung der Gesellschaft. Und bezogen auf soziale Gleichheit müssen wir, wenn wir uns in der Welt umschauen, konstatieren, mit welchem großen Maß an sozialer Ungleichheit funktionierende Staatswesen möglich sind. Probleme sozialer Homogenität können in jeweiligen historischen Konstellationen auch unabhängig von Schichten- oder Klassenaspekten erwachsen - etwa auch in Kontexten, die zum Thema von heute morgen gehören. Fazit also: In einem sehr generalisierenden Sinne ist ein gewisses $\mathrm{Ma} ß$ an sozialer Homogenität Verfassungsvoraussetzung. Wenn ich das Ziel sozialer Gleichheit aber konkret auf den Abbau oder auch nur die Milderung sozialer Schichtung beziehe, muss die gestellte Frage wohl verneint werden.

Zum Thema der sozialen Gleichheit als Verfassungsaufgabe sieht es ähnlich aus. Einerseits geht es um die Sozialstaatlichkeit als Staatsziel, also eine durch die Verfassung vorgegebene Aufgabe, soziale Homogenität und Integration zu fördern. Andererseits lassen sich daraus aber kaum konkrete rechtliche Antworten im Themenfeld sozialer Gleichheit gewinnen. Beim Existenzminimum zum Lebensunterhalt und auch beim gesundheitlichen Existenzminimum steht die Verfassungsgarantie der Menschenwürde im Mittelpunkt, allerdings wohl eher deren objektiv-rechtliche Dimension: Ein Gemeinwesen, in welchem behandlungsbedürftige Kranke mangels finanzieller Mittel vor die Tür eines Krankenhauses gesetzt werden dürfen oder in welchem aus Armut gehungert wird, kann sich kaum rühmen, die gleiche Würde aller Menschen zu seinem obersten Prinzip gemacht zu haben. Ganz schwierig ist es bei einem vergleichbar wichtigen Thema: der Chancengleichheit der Jugend im Hinblick auf ihre Lebensperspektiven. Das betrifft vor allem die Bildungschancen, aber auch in deren Vorfeld liegende Erziehungschancen. Abgesehen von Extremfällen, in denen das Wächteramt des Staates gefordert ist, kann der Verfassungsauftrag für soziale Gleichheit keine griffigen Kriterien liefern. Auch hier wäre zu fragen: Sind es nicht eher die objektiv-rechtlichen Dimensionen von Grundrechten, die hier weiterhelfen? Wäre unser Staatwesen auf die gleiche Würde für alle ausgerichtet, wenn die individuellen Entwicklungschancen von Kindern dadurch determiniert würden, wohin sie geboren wurden? Verlangt nicht das Recht auf freie Entfaltung der Persönlichkeit gegebenenfalls stützende staatliche Aktivitäten, um dieses Recht zu einem gleichen Recht aller zu machen?

Pitschas: Offenbar waren nicht alle der hier Mitdiskutierenden im Saal, als die Vorträge gehalten worden sind, denn man muss doch feststellen, dass beide Referenten - Herr Ebsen wies eben darauf hin - also sowohl 
die Referentin als auch der Referent, die Themenfrage beantwortet haben, ja mehr noch eigentlich, die Validität der Themenfrage bejahten. Wendet man sich auf der Grundlage des Themas zunächst dem Erstreferat $\mathrm{zu}$, dann finde ich ganz hervorragend, dass auch die internationalen Bezüge sozialer Gleichheit hervorgehoben worden sind und Frau Davy darüber hinaus noch hätte weiter ausgreifen können, etwa auf die soziale Dimension der WTO-Diskussion und dort den Aspekt der Forderung nach sozialer Gleichheit hätte bestätigen können. Auch die Darlegungen zum Europäischen Sozialrecht fand ich sehr erhellend, insbesondere, dass die Konzeption der „social citizenship“ erwähnt worden ist. Man könnte vielleicht hinzufügen, dass es ja zwei Konzepte gibt, nämlich daneben das Konzept der Antidiskriminierung, das in den Vorstellungen der Kommission nunmehr und im Zusammenhang mit ihrer sozialen Agenda im Juli 2008 zu dem Entwurf einer dritten Antidiskriminierungsrichtlinie geführt hat. Dabei wurde die Frage der „social citizenship“ mit Absicht beiseite gerückt, weil daraus gewisse aktive Förderungspflichten gegenüber sozialer Ungleichheit entstehen, die Frau Davy auch angesprochen hatte.

Ich möchte allerdings drei Punkte hinzufügen, die mir nicht so unkritisch erscheinen wie der Referentin. Da ist zunächst die Ausgangsfrage: Ist soziale Gleichheit eine Voraussetzung der Verfassung? Ich würde diese Frage bejahen. Jede moderne Verfassung muss auf wohlfahrtsstaatliche Aspekte Wert legen, um ganz im Sinne Hermann Hellers eine gewisse Mindestintegrationskraft zu entfalten. Geschieht dies nicht, ist die Verfassung nicht das Papier wert, auf dem sie geschrieben steht. Und schon Herr Häberle hat viele Jahre stets darauf hingewiesen, dass die Verfassungen der Entwicklungsstaaten aus diesem Grund immer wieder die „soziale Gleichheit“ thematisiert haben, allerdings - da hat Frau Nußberger Recht - ausgehend von dem Aspekt des Sozialstaates als Staatsziel. Wenn man die Gleichheit i. S. sozialer Gleichheit als Verfassungsvoraussetzung bejaht, dann entbindet das - Frau Davy, Sie entschuldigen bitte, dass ich darauf eingehe - bei den drei Beispielsfällen nicht von der Nachfrage, ob es eine grundrechtsdogmatische Verbindung etwa von Art. 12 zu Art. 3 Abs. 1 GG gibt und sich daraus soziale Gleichheit speist. Oder steht Art. 2 Abs. 1 GG im Fall Gesundheit in Verbindung mit Art. 2 Abs. 2 Satz 1 GG, so dass wir, glaube ich, immer sehen müssen, dass bei komplexen Lagen die grundrechtlichen Freiheitsrechte in einer engen Grundrechtsverbindung wirken? So gibt es denn auch Rechtsprechung, die etwa die Geltung von Art. 3 Abs. 3 GG im Recht der behinderten Menschen mit anderen Grundrechtsaussagen wie z.B. zu Art. 6 Abs. 1 GG unterfüttert. Herr Axer hat im Übrigen in seinen Ausführungen - und damit komme ich ja auch schon zum 
Schluss - in ganz besonders hervorragender Weise, wie ich meine, gezeigt, dass in jedem Satz wohlabgewogen die Grundrechtsdiskussion um soziale Gleichheit aufgenommen wird. Das hat mir sehr gut gefallen.

Zacher: Ich möchte zunächst aus vollem Herzen dem Vorstand danken, dass er dieses Thema auf die Tagesordnung gesetzt hat. Denn es ist in der Tat für alle unsere Gesellschaften ein ganz zentrales Thema und auch für unser Verfassungsleben. Ich habe mich sehr gefreut darüber, dass das jetzt hier behandelt worden ist. Und ich danke natürlich auch den beiden Referenten. Ich möchte mich nun nicht in Einzelkritik vertiefen, sondern ein paar Punkte zur Sprache bringen, von denen ich glaube, dass sie in den beiden Referaten durchaus angeklungen sind, dass sie aber noch schärfer hätten artikuliert werden können.

Der erste Punkt ist der, dass alle Gesellschaften dieser Welt heute in sich damit ringen, dass es Kräfte gibt, die radikal soziale Gleichheit wollen, dass es aber auch Kräfte gibt, die, selbst wenn sie Gleichheit wollen, auch Ungleichheit bejahen - vor allem die „glückliche Ungleichheit“ durch Leistung und Glück -, und dass es schließlich Kräfte gibt, die keine Gleichheit wollen. Und noch unendlich mehr gehen die Standpunkte darüber auseinander, was das konkret bedeutet. Die Gesellschaften haben es schwer, hier Konsense zu finden. Ja, es ist fast immer ausgeschlossen, Konsense zu finden. Somit liegt eine ganz zentrale Aufgabe des Staates darin, eine Linie zu finden zwischen all den Dissensen, zwischen einem Grundkonsens, vielleicht auch einer Grundgegebenheit, dass es Gleichheit geben soll, aber auch Ungleichheit geben soll. Der Staat muss die möglichen Kompromisslinien suchen und finden. Das ist, glaube ich, eine zentrale Legitimationsfrage für den Staat. Von daher gibt es eine klare Antwort auf die Frage des Vorstands nach dem Ort der Gleichheitsfrage: Voraussetzung oder Auftrag? Indem dem Staat die Antwort auf die Gleichheitsfrage gelingt, integriert er die Gesellschaft und legitimiert er sich der Gesellschaft gegenüber. Indem ihm die Antwort misslingt, delegitimiert sich der Staat. Wir wissen: Auf Fragen der Legitimation und Legitimation von Verfassung und von Regierung gibt es keine positiv-rechtliche Antwort. Aber wir erleben alle Tage, wie kritisch die Situation werden kann, wenn die Legitimation misslingt. Diese Kompromisslinie zwischen Gleichheit und Ungleichheit zu finden, ist eine zentrale Aufgabe der modernen Staaten, so auch der Bundesrepublik; und im Verfassungsstaat ist es somit auch eine Herausforderung an die Verfassung. Sie hat den Staat so einzurichten, dass er diese Aufgabe erfüllen kann.

Das zweite, was ich sagen wollte, ist, dass diese Linie immer nur auf Zeit gezogen werden kann; denn sie wird nie endgültige Zufriedenheit 
auslösen. Es wird immer, früher oder später, Menschen geben, die sagen: Diese Kompromisslinie, so wie sie aktuell gesetzt ist, ist ungerecht; da ist Gleichheit vernachlässigt; da ist Ungleichheit nicht ausgeglichen usw. Das wird es immer geben. Die Aufgabe, die Kompromisslinie zu ziehen, wird sich nie endgültig lösen lassen. Damit komme ich gleich zum dritten. Weil das so ist, wird es auch keine definitive Antwort, keine normative Antwort in der Verfassung oder aus der Verfassung geben können. Und das ist das, was ich bei allen heute vermisst habe und was ich für so wichtig halte: Den Staat zu befähigen, die Kompromisslinie immer neu zu setzen, ist eine verfassungspolitische Aufgabe. In der Diskussion sind die Dinge angeklungen: Wahlrecht usw. Da wäre noch mehr zu sagen. Jedenfalls, die Organisation der Politik durch die Verfassung muss dieser Aufgabe gerecht werden. Roman Herzog hat vor kurzem mit Recht das Mehrheitswahlrecht ins Spiel gebracht. Das ist das Muster, von dem her die Frage, die der Vorstand gestellt hat, diskutiert werden muss.

Holoubek: Wir kommen jetzt zu Herrn Häberle, der eine verfassungsvergleichende Umschau angekündigt und gleichzeitig versprochen hat, nicht mehr als vier Minuten dafür zu benötigen.

Häberle: Erlauben Sie eine kleine verfassungsvergleichende Umschau, die die eindrucksvollen Referate nur unterfüttern und unterstützen soll, nicht mehr. Ein Blick auf vier Länder: Erstens, angedeutet von Herrn Axer, aus dem ungeschriebenen Recht auf wirtschaftliches Existenzminimum des Schweizer Bundesgerichts in Lausanne hat die Bundesverfassung von 1999 ein geschriebenes Verfassungsrecht gemacht, und viele vortreffliche totalrevidierte Kantonsverfassungen in der halben Schweiz sind alldem schon vorausgegangen oder später nachgefolgt. Ich erwähne erneut preisend mit viel hohen Tönen die „Werkstatt Schweiz“, nämlich deren Verfassungstexte zur Solidarität mit den Hilfsbedürftigen in der Welt: etwa in der vortrefflichen Berner Verfassung von 1993 aus der Feder von Jörg Paul Müller und vielen anderen, und auch in der Bundesverfassung, jeweils Art. 54 Absatz 2.

Zweitens: Ein Blick auf Italien und Spanien. Die Italiener sind stolz darauf - es ist wohl Art. 3 Satz 2 ihrer Verfassung von 1947 -, dass sie den Leitsatz 15 von Herrn Axer vorweg genommen haben, denn dort ist in etwa gesagt: „Alle genießen dieselbe soziale Achtung, der Staat soll die Hindernisse wirtschaftlicher und gesellschaftlicher Art beseitigen, die die Freiheit und Gleichheit der Bürger tatsächlich einschränken und die wirksame Teilnahme aller an der Gestaltung des Landes verhindern.“ Dies hat Spanien in seiner Verfassung von 1978 in Art. 9 Abs. 2 einfach 
rezipiert: Freiheit und Gleichheit der einzelnen Gruppen sollen „real und wirksam“ sein, Hindernisse für ihre volle Entfaltung sollen beseitigt werden, um Teilnahmechancen zu eröffnen.

Drittens: Ein Blick auf die Schwellen- und Entwicklungsländer - Herr Pitschas hat soeben darauf hingewiesen. Wenn man sich einige neuere Verfassungen und Verfassungsentwürfe vergegenwärtigt, mir sind die alte Verfassung von Peru von 1979 und der neue dortige Verfassungsentwurf, der bislang steckengeblieben ist (nach der Zwischenverfassung von Fujimori) präsent, auch die Guatemala-Verfassung von 1985, die ich vor zehn Jahren schon einmal zur großen Heiterkeit unseres Plenums zitiert habe. In diesen Textstufen wird deutlich zum Ausdruck gebracht, dass die Schaffung der sozialen Gleichheit dazu dient, soziale Integration zu ermöglichen, um das Ganze zusammenzuhalten. Es finden sich etwa soziale Mindestrechte, auch in Sachen Kultur, Bildung, Gesundheit und Arbeit. Im Grunde geht es um den Gedanken von Hermann Heller und das, was Herr Zacher soeben geäußert hat. All diese Textstufen empfinden die Entwicklungsländer und Schwellenländer sowohl in der Verfassungsrechtswissenschaft als auch im Alltag, sowohl in der Politik als auch in der Verfassungspolitik, als zentral für die Versöhnung von Freiheit und Gleichheit. Dies ist ein unendlicher Prozess, freilich auch mit vielen Rückschlägen, wie man beim Studieren von Argentinien, Peru und Brasilien beispielhaft erleben kann. Herr Vorsitzender, ich stelle fest: Bei „Ampelgelb“ höre ich auf. Vielen Dank.

Holoubek: Herr Häberle, es ist faszinierend, eine Weltreise in drei Minuten; wir danken ganz herzlich.

Tomuschat: Einige Redner haben schon darauf hingewiesen, dass das Thema in einem größeren Kontext gesehen werden muss, und einer dieser Faktoren ist das gegenseitige Verhältnis von liberalen Rechten zu sozialen Rechten. Ich finde, dass in den Vorträgen davon nicht ausreichend die Rede war. Meines Erachtens setzt das Grundgesetz ganz eindeutige Akzente in den Art. 1, 2 Abs. 2, 9, 12 und 14 GG: Der Mensch soll in erster Linie für seine eigene Existenz sorgen. Herr Zacher hat zu Recht gesagt, dass der Staat sich auch durch Wohlfahrtsleistungen legitimieren muss, aber der Staat muss seine Hand dem Schwachen hinhalten, er soll nicht die Mehrheit der Bevölkerung oder einen Großteil der Bevölkerung in die Unmündigkeit führen. Niemand sollte sein Leben in Hartz IV einrichten. Es kann nicht der Sinn sozialer Gleichheit sein, dass man Menschen mit irgendwelchen sozialen Leistungen abspeist. Es gilt vielmehr, strukturelle Reformen in die Wege zu leiten. Herr Morlok hat dies auch schon gesagt, ebenso Herr Ebsen. Nach meiner Auffas- 
sung sind vor allem Erziehung und Bildung das Instrument, mit dem die bestehenden strukturellen Ungleichheiten überwunden werden können, und zwar durch die Anstrengungen der Menschen selbst. Diese brauchen nicht staatliche Hilfsleistungen ex post, sondern man muss ihnen rechtzeitig Chancen geben. Herr Axer hat das alles durchaus zutreffend ausgeführt, aber in der Fülle seiner Erwägungen sind diese Zentralpunkte etwas untergegangen. Ich möchte nachdrücklich der These 9 widersprechen, wo gesagt wird, gleiche rechtliche Freiheit erzeuge soziale Ungleichheit. Sie erzeugt in erster Linie soziale Gleichheit, weil der Art. 3 GG nämlich das Vehikel ist, um sozialen Aufstieg zu sichern, soziale Mobilität. Meines Erachtens ist das, was dort gesagt wird - und ich möchte auch Herrn Meyer widersprechen, vehement sogar widersprechen - völlig ahistorisch. Denken Sie an Leibeigenschaft und denken Sie an Sklaverei, alle diese Dinge einer gar nicht so entfernten Vergangenheit. Diese Missstände sind beseitigt worden durch gleiche Rechte, durch die Gleichheit, die sich sukzessive durchgesetzt hat. Abermals betont sei aber, dass es die Kräfte des Einzelnen selbst sind, die ihn in die Lage versetzen, seine Situation zu verbessern. Er braucht nicht die meist geringen sozialen Leistungen des Staates. Wenn er freilich in Armut fällt, wenn er nicht selbst für seine Situation sorgen kann, dann natürlich soll der Staat ihm unter die Arme greifen. Der Staat soll aber nicht - ich wiederhole es - einen Großteil der Bevölkerung in die Unmündigkeit führen.

Hufen: Die Regie fügt es sehr passend, dass ich an Herrn Tomuschat und teilweise auch an Herrn Ebsen und Herrn Morlok anknüpfen kann und auf die Brücken trete, die der Vorstand mit dem schönen Thema zwischen den unterschiedlichen Aspekten von Kultur und sozialer Gerechtigkeit errichtet hat. Das dahinterstehende Thema lautet ja immer: Welche Voraussetzungen muss der freiheitliche Verfassungsstaat in der sozialen und kulturellen Wirklichkeit schaffen, damit er nicht erodiert? Gewiss gehört zu diesen Voraussetzungen ein bestimmtes Maß an sozialer Chancengleichheit und die ist ja bekanntlich nicht identisch mit Verteilungs- oder gar Ergebnisgleichheit. Wir haben jetzt gerade wieder von Herrn Tomuschat sehr nachdrücklich gehört, dass die Methode der Gießkanne, die Methode der Umverteilung, die Methode der zwangsweisen Schließung von sozialen und wirtschaftlichen Scheren und Ähnliches in die Irre führt und stets neue Ungleichheit und Unmut erzeugt. Der wirkliche Weg zu mehr Gerechtigkeit, um den es hier geht, muss ganz früh ansetzen, und ganz früh heißt: Schule, heißt: Erziehung. Das hat Herr Axer in einer These ganz leicht anklingen lassen - aber im Übrigen schien mir dieser Aspekt sozialer Gleichheit doch in beiden Refe- 
raten zu wenig beleuchtet. Auch in der Schule gibt es eine Methode der schematischen Umverteilung, die kein Königsweg sein kann: Chancenvermehrung der Einen durch Chancenminderung der anderen. Ich will mal ganz plakativ sagen, spätestens bei sechs gemeinsamen Grundschuljahren hört die Verfassungsmäßigkeit der Gleichstellung hier auf. Eine obligatorische Gesamtschule ohne Leistungsdifferenzierung und ähnliches kann also nicht die richtige Antwort sein. Der Blick muss viel früher ansetzen, zwangsläufig auf die ganz frühe Phase fallen, und das heißt eben Kinderbetreuung, das heißt Ganztagsvorschule, Ganztagsgrundschule, die Ermöglichung von Kompensation im frühen Stadium, damit wir eben hier Chancenungleichheiten der sprachlich und sozial benachteiligten Kinder beseitigen und die bereits mehrere Generationen umfassenden Sozialhilfe- und Hartz IV-Karrieren an der Stelle unterbrechen, wo es vielleicht noch möglich ist. Es gibt auch in diesem Kreis Kolleginnen und Kollegen, die immer noch sagen, eine Ganztagsschule im Grundschulbereich oder gar eine obligatorische Vorschule nach dem Modell der erfolgreichen französischen „école maternelle“ wären sogar verfassungsrechtlich bedenklich. Die Sorgen möchte ich haben! Ich habe vielmehr die Sorge, möglichst viele benachteiligte Kinder möglichst früh in die Schule zu bekommen. Vielen Dank.

Welti: Die Referate und auch die Diskussionen haben bereits gut herausgearbeitet, dass das Thema nicht soziale Gleichheit im Sinne von Gleichverteilung ist, sondern dass es jene Voraussetzungen des Staates auszuloten gilt, die ein Mindestmaß ermöglichen: Ein Mindestmaß an sozialer Teilhabe - wenn man es individualrechtlich sieht, an Integration - staatsrechtlich gedacht, an Inklusion - soziologisch oder an sozialer Kohärenz - europarechtlich ausgedrückt. Und sozial in diesem Sinne ist eben nicht nur Wieselwort, sondern es verweist auch auf das, worum es zunächst geht: nämlich auf die Gesellschaft und darauf, dass einem funktionierenden Staat eine funktionierende Gesellschaft gegenüber zu stehen hat, in der die Menschen als gleichberechtigte Staatsbürger Demokratie ausüben können und sich als gleichberechtigte Vertragspartner gegenüberstehend einen Rechtsstaat konstituieren können. Das ist das Maß an sozialer Gleichheit, das hier gesucht wird und das übrigens auch nicht in Chancengleichheit aufzulösen ist oder Bildungsaufstieg. Man muss auch in Würde am Aufstieg scheitern können. Die Frage, welche Schwerpunkte der Staat hier zu setzen hat, was seine Aufgaben hier sind, ist seit 1994 in Deutschland verfassungsrechtlich besser akzentuiert worden mit den besonderen Gleichheitssätzen. Der Staat hat in die gesellschaftliche Wirklichkeit hinein die Gleichstellung der Geschlechter und auch die Gleichheit der behinderten Menschen ins 
Auge zu nehmen, d.h. die besonderen Gleichheitssätze setzen einen verfassungsrechtlichen Haltepunkt dafür, wo der Staat gefordert ist, auch soziale Voraussetzungen zu schaffen. Niemand darf wegen seiner Behinderung benachteiligt werden. Hier ist eine Gruppe von Millionen Menschen in unserer Gesellschaft bezeichnet, die tatsächlich soziale Unterstützung benötigt, nicht nur durch Sozialleistungen, auch durch Anti-Diskriminierungs-Gesetzgebung, um dann tatsächlich die Grundrechtsvoraussetzungen gesichert zu bekommen. Als Banker hat und bekommt man ohnehin große Mengen Geldes, um seine Grundrechtsvoraussetzungen gestützt zu bekommen, nein, es geht um diese Menschen, die tatsächlich die Unterstützung des Gemeinwesens benötigen.

Konkrete Frage an Frau Davy: Sie hatten die Probleme der sozialen Gleichheit im Zusammenhang mit dem pauschalierten Existenzminimum benannt, auch bei den Gesundheitsleistungen. Die soziale Gleichheit behinderter Menschen ist ein gutes Beispiel, wie man auch grundrechtsgestützt mit Hilfe der besonderen Gleichheitssätze feststellen kann, dass Gleichheit der sozialen Teilhabe auch bedeutet, dass der Staat die Ungleichheit der Voraussetzungen anerkennen muss. Wenn ich ein gleiches Existenzminimum bei Gesundheitsleistungen oder auch bei Rentenleistungen auf diejenigen anwende, deren Voraussetzungen eben andere sind, die ich nicht auf die Selbsthilfe verweisen kann beim Kauf einer Sehhilfe oder auch beim Erwerb von Rentenansprüchen, dann muss ich anders mit ihnen umgehen, und hier ist dann die Pauschalierung im SGB II oder auch SGB XII verfassungsrechtlich bedenklich.

Fehling: Ich möchte einige kurze Anmerkungen zum Gestaltungsspielraum des Gesetzgebers machen, also zum Spielraum bei der Frage, wie viel Ungleichheit noch hinnehmbar ist. Dieser politische Spielraum hat nämlich - und das scheint mir wichtig - zwei Richtungen. Die eine Richtung stand in den Referaten - besonders, glaube ich, bei Frau Davy, aber auch bei Herrn Axer - sehr stark im Vordergrund, wohl nicht zuletzt wegen der politischen Entwicklungen der letzten Jahre: Wie weit kann man soziale Standards absenken, ohne in die Zone der Verfassungswidrigkeit abzurutschen? Hier stimme ich im Grundsatz mit den Referenten überein, dass insoweit der Gesetzgeber - gerade weil es keinen politischen Konsens in der Gesellschaft über das erträgliche $\mathrm{Ma} ß$ sozialer Ungleichheit gibt - einen sehr breiten Spielraum besitzen muss. Ich bin aber nicht ganz sicher, ob die Referenten, vor allem Frau Davy bei ihrer These 6 , hier nicht doch etwas übertrieben haben. Wenn es multiple soziale Exklusion gibt, dann ist doch noch einmal nachzufragen: Müssen wir nicht vor der Menschenwürde das Existenzminimum auch als soziales und nicht nur als Überlebensminimum physischer Art 
definieren und muss es dann nicht auch ein Verfahren geben, um gegebenenfalls den Ball an den Gesetzgeber zurückzuspielen? Es geht ja nicht darum, dass ein Gericht Vorgaben macht, wie im einzelnen soziale Exklusion zu verhindern ist. Aber ein Gericht muss beanstanden können, dass das soziale Netz so eben nicht ausreicht, um Mindeststandards an gesellschaftlicher Teilhabe zu wahren.

Dies ist die eine Seite, aber die andere Seite ist mir eigentlich noch wichtiger. Ein wenig klingt mir dies schon bei dem an, was Herr Morlok gesagt hat: Wie steht es mit dem Spielraum des Gesetzgebers, bei vielleicht anderen politischen Mehrheiten das Ruder herumzureißen, also aktiv daran zu arbeiten, soziale Ungleichheiten abzubauen? Das kann durch die viel gescholtene Umverteilung geschehen, aber natürlich auch durch das Bildungssystem, gegebenenfalls sogar durch Änderungen im Wirtschaftssystem. Letztendlich ist es eben auch eine politische Frage: Was will man und was will man nicht? Je nach Standpunkt kann ein geringeres oder ein höheres Maß an sozialer Gleichheit als Grundrechtsvoraussetzung und auch als Verfassungsvoraussetzung - Hermann Heller wurde ja mehrfach genannt - geboten erscheinen. Wenn man auf der einen Seite den Gestaltungsspielraum des Gesetzgebers massiv betont, nämlich bei der Möglichkeit, Standards abzusenken, dann muss man umgekehrt auch darüber nachdenken, ob der Gesetzgeber nicht ebenso breite Gestaltungsspielräume beanspruchen kann, um bei anderen Mehrheiten auch anders reagieren zu können. Insofern sind vielleicht im Steuerrecht und anderen Rechtsgebieten bisher die Freiheitsrechte doch zu stark gemacht worden, um einen Wall gegen entsprechendes Umsteuern zu errichten. Das ergäbe dann aus meiner Sicht eine bedenkliche Asymmetrie.

Iliopoulos-Strangas: Ich möchte der ersten These von Frau Davy, dass nämlich soziale Gleichheit keine Aufgabe einer Verfassung sei, entschieden widersprechen. Richtig ist vielmehr: Was Aufgabe einer Verfassung sein soll, ist von der jeweiligen Verfassung selbst zu bestimmen. Diese Aufgabenzuweisung braucht nicht ausdrücklich zu erfolgen. So kann etwa in Rechtsordnungen wie derjenigen des deutschen Grundgesetzes, welche die Sozialstaatsklausel und den Gleichheitssatz verfassungsrechtlich garantieren, ohne Weiteres aus solchen Verfassungsbestimmungen geschlossen werden, dass hier soziale Gleichheit zur Verfassungsaufgabe gemacht wurde. Ergänzend zu Herrn Häberle möchte ich die Rechtsvergleichung heranziehen, um weitere Beispiele von Verfassungen oder auch Verfassungsrechtsprechung anzuführen, die meine Position bestätigen. So geht die griechische Verfassung weiter als das Grundgesetz, indem sie neben dem Gleichheitssatz und der Sozial- 
staatsklausel eine Reihe weiterer Bestimmungen enthält, aus denen sich folgern lässt, dass soziale Gleichheit Gegenstand einer wie auch immer zu erfüllenden Verfassungsaufgabe sein kann. In der Tat wird in Artikel 25 Abs. 2 der griechischen Verfassung proklamiert, dass die Anerkennung und der Schutz der grundlegenden und immerwährenden Menschenrechte durch den Staat auf die Verwirklichung des gesellschaftlichen Fortschritts in Freiheit und Gerechtigkeit gerichtet ist; des Weiteren wird im vierten Absatz derselben Verfassungsbestimmung festgelegt, dass der Staat berechtigt ist, von allen Bürgern die Erfüllung ihrer Pflicht zu gesellschaftlicher und nationaler Solidarität $\mathrm{zu}$ fordern. Ferner könnte man auf die Regelung im zweiten Absatz der obigen Verfassungsbestimmung hinweisen, wonach die Grund- und Menschenrechte auch in den Beziehungen zwischen Privaten gelten, soweit sie sich dafür eignen (verfassungsrechtliche Absicherung der Drittwirkung). Deuten alle diese Bestimmungen in Verbindung mit dem Gleichheitssatz (Art. 4 gr. Verf.) und der Sozialstaatsklausel (Art. 25 Abs. 1 gr. Verf.) nicht darauf hin, dass die soziale Gleichheit zu den Aufgaben der griechischen Verfassung zählt?

Für die These, dass soziale Gleichheit Aufgabe einer Verfassung sein kann, sprechen außerdem zwei weitere Argumente. Zum einen weisen in diese Richtung auch die in einer Reihe von Verfassungen enthaltenen Bestimmungen, welche die so genannte affirmative action (positive Diskriminierung) institutionalisieren und damit dem Staat mittelbar die verfassungsrechtliche Aufgabe auferlegen, (zumindest) in begrenzten Bereichen für soziale Gleichheit zu sorgen. Zum anderen ist jene Rechtsprechung von Verfassungsgerichten und sonstigen Höchstgerichten zu nennen, die den Gleichheitssatz als Vehikel benutzt, um soziale Gleichheit herzustellen: sei es durch die Ableitung von derivativen sozialen Grundrechten aus dem Gleichheitssatz bzw. dem Diskriminierungsverbot (so etwa der österreichische Verfassungsgerichtshof), sei es - im Falle der Feststellung eines Verstoßes gegen den Gleichheitssatz - durch Erstreckung begünstigender Maßnahmen bzw. sozialer Leistungen, die vom Gesetzgeber nur einem bestimmten Personenkreis gewährt wurden, auf nicht begünstigte, insbesondere willkürlich ausgeschlossene Personengruppen (so etwa die Verfassungsrechtsprechung in Belgien, in Griechenland, in Italien, in den Niederlanden, in Österreich und in Spanien). Man kann sich schwerlich der Erkenntnis verschließen, dass in den obigen Fällen die soziale Gleichheit als Aufgabe der Verfassung verstanden wird. Kurz gesagt, sehe ich nicht ein, wie die soziale Gleichheit jedenfalls in Verfassungen, die die Sozialstaatsklausel und den Gleichheitssatz garantieren, nicht als Aufgabe der Verfassung angesehen werden könnte. 
Ich komme jetzt zu der These Nr. 13 von Herrn Axer. Unbeschadet der von mir bereits erwähnten Rechtsprechung staatlicher Gerichte zu den Rechtsfolgen eines Verstoßes gegen den Gleichheitssatz stimme ich Ihnen, Herr Kollege Axer, zu, dass sich originäre Ansprüche auf bestimmte Maßnahmen zur Herstellung sozialer Gleichheit grundsätzlich nicht aus dem sozialen Staatsziel - selbst in Verbindung mit dem allgemeinen Gleichheitssatz - herleiten lassen und dass sich stattdessen im Einzelfall aus den Grundrechten Ansprüche auf eine Mindestsicherung ergeben können, etwa auf die Gewährleistung des Existenzminimums. Ich frage mich allerdings, ob solche Ansprüche auf Mindestsicherung und Existenzminimum jeweils ausschließlich auf einzelne Grundrechte zu stützen sind. Könnte gegebenenfalls nicht auch die Menschenwürde eine gewisse Rolle in dieser Frage spielen, wie es manche meiner Vorredner bereits angedeutet haben? Darüber hinaus halte ich es für dogmatisch überzeugender, in Rechtsordnungen, die eine grundrechtliche Wesensgehaltsgarantie kennen, verfassungsrechtliche Ansprüche auf Mindestsicherung nicht allein den Einzelgrundrechten zu entnehmen, sondern sie zugleich in der Wesensgehaltsgarantie zu verorten. Die Wesensgehaltsgarantie als Schranken-Schranke könnte nämlich in ähnlicher Weise wie die einzelnen Abwehrrechte nicht nur negativ, sondern auch in dem Sinne positiv gedeutet werden, dass der Staat verpflichtet ist, durch aktives Tun einen Mindestschutz des jeweiligen Grundrechts sicherzustellen. In diesem Sinne erscheint es mir vorzugswürdig, als verfassungsrechtliche Grundlage für Ansprüche auf eine Mindestsicherung im deutschen und spanischen Recht die - wohl im Unterschied zu den meisten anderen Staatsverfassungen - im deutschen Grundgesetz (Art. 19 Abs. 2) und in der spanischen Verfassung (Art. 53 Abs. 1) ausdrücklich enthaltene Wesensgehaltsgarantie in Verbindung mit dem jeweiligen Grundrecht heranzuziehen.

Emmerich-Fritsche: Sie sagen, Frau Davy, soziale Gleichheit ist weder Voraussetzung noch Aufgabe einer Verfassung. Mein erstes Problem ist: Was ist soziale Gleichheit? Es gibt offensichtlich eine rechtliche Dimension dieses Begriffs und diese ist wohl doch Aufgabe der Verfassung. Sie haben das gezeigt am Beispiel der Menschenwürde. Es wurden weiterhin angeführt das Recht auf Bildung, das Diskriminierungsverbot, das Sozialstaatsgebot, zumindest Chancengleichheit wurde ergänzend erwähnt, und man könnte vielleicht auch die soziale Dimension der Grundrechte hinzunehmen sowie die Schutzpflichtlehre.

Mich interessiert vor allem die europäische und die internationale Dimension. Zu Recht haben Sie den Begriff der Verfassung als mehrgliedrigen Begriff aufgefasst. Sie sind also vom Grundgesetz über die euro- 
päische Verfassung bis hin zu einer internationalen oder globalen Verfassung gegangen - wobei in Bezug auf letzteres das Wort Verfassung vielleicht noch mit einem Fragezeichen zu versehen ist. Es ist interessant zu sehen, dass die Arbeitnehmerfreizügigkeit, auch die Unionsbürgerschaft diesen Begriff der sozialen Ungleichheit nicht in erster Linie zu dem Zweck von mehr Selbstbestimmung, sondern eigentlich zu dem Zweck der Integration verrechtlicht haben. Inhalt der Grundfreiheiten ist ja nicht nur ein Diskriminierungsverbot, sondern jede faktische Beschränkung, und dazu gehören auch ungleiche soziale Rahmenbedingungen. Über die Grundfreiheiten wird der Begriff der sozialen Gleichheit europarechtlich weiter verrechtlicht, als dies nach dem Grundgesetz der Fall ist, aber zu einem anderen Zweck, nämlich zum Zweck der Harmonisierung der Wettbewerbsbedingungen und zur Verwirklichung des Binnenmarktes.

Ein anderer Aspekt, der noch als großes Leerfeld erscheint, ist die globale soziale Ungleichheit. Hier verwenden wir viele weiche Begriffe und stoßen auf Unklarheiten. Das liegt auch daran, dass der Begriff soziale Gleichheit als Rechtsbegriff mehr Bedeutung bekommt, je enger und homogener die Rechtsgemeinschaft ist. Auf der globalen Verfassungsebene gibt es diese Homogenität nicht, und deswegen haben wir hier kaum Möglichkeiten, eine rechtliche Grundlage für soziale Gleichheit zu finden. Ihr Ansatzpunkt social security oder responsibility to protect sind weite Modelle mit noch wenig rechtlich ausgefülltem Hintergrund. Auf Weltebene gibt es nur die Solidarität zwischen Menschen. Insoweit bietet sich also die Menschenwürde als rechtlicher Grund sozialer Gerechtigkeit an. Meine Frage: Ist die Menschenwürde aus Ihrer Sicht für die Weltgemeinschaft eine Aufgabe oder ein taugliches subjektives Recht, ein Gewohnheitsrecht oder allgemeiner Rechtsgrundsatz, der zum jus cogens zählt? Könnte man die Menschenwürde als obersten Verfassungsgrundsatz dieser globalen Verfassung ansehen und damit die responsibility to protect stärken? Erlauben Sie mir noch eine zweite Bemerkung: Die WTO zeigt unter dem verfassungsrechtlichen Aspekt eine extreme Asymmetrie. Verrechtlicht ist nur die Waren- und Dienstleistungsfreiheit, nicht die Arbeitnehmerfreizügigkeit. Und eine soziale Dimension fehlt im WTO-Recht völlig.

Holoubek: Wir sind damit zum Abschluss der Diskussion bei Wortmeldungen angelangt, die verstärkt den Blickwinkel auf europäische und internationale Aspekte werfen wollen.

Groß: Ich möchte nachhaken bei dem Thema der Existenzsicherung, das in beiden Referaten angesprochen worden ist, und zwei konkrete 
Gruppen ansprechen, wo sich, glaube ich, konkret die Frage stellt, ob das Existenzminimum rechtlich gesichert ist, und daraus eine allgemeine Schlussfolgerung ziehen. Wenn ich mir überlege, bei welchen Gruppen die auch rechtlich abgesicherte soziale Sicherung am geringsten ist, dann lande ich bei zwei Ausländergruppen. Das eine sind die Asylbewerber, die unter das Asylbewerberleistungsgesetz fallen, was seit ungefähr 15 Jahren einen gegenüber dem damaligen Bundessozialhilfegesetz und jetzigen SGB II noch mal deutlich abgesenkten Regelsatz vorsieht. Noch problematischer ist, dass dieser Regelsatz seit etwa 15 Jahren nie angehoben worden ist, so dass sich hier noch deutlicher die Frage stellt, wo eigentlich die Untergrenze einer sozialen Unterstützung ist.

Die zweite Gruppe ist die Gruppe der illegal in Deutschland Lebenden, deren Zahl auf mehrere Hunderttausend geschätzt wird von Leuten, die in dem Bereich arbeiten. Sie bekommen natürlich keinerlei rechtlich abgesicherte Unterstützung. Dort stellt sich aber besonders brennend die Frage der gesundheitlichen Mindestversorgung. Aufgrund der Meldepflichten, die bestehen, müssen sie nämlich aufenthaltsbeendende Maßnahmen fürchten, wenn sie sich an irgendeine staatliche Stelle, damit auch an viele Krankenhäuser wenden, was dazu führt, dass sie sich meistens nur bei Lebensgefahr überhaupt in ärztliche Behandlung begeben, abgesehen von ein paar freiwillig und völlig ohne staatliche Unterstützung in dem Bereich tätigen Ärzten, die sich dieser Gruppe widmen. Auch dort stellt sich die Frage: Gibt es im gesundheitlichen Bereich einen Bereich des Existenzminimums, der jedenfalls nicht einer so hohen faktischen Hürde unterworfen werden darf, wie sie durch das Aufenthaltsrecht geschaffen wird?

Wenn man sich diesen konkreten Problemen stellt, kommt man zur Frage: Wo liegt eigentlich die rechtliche Basis und warum haben wir so große Scheu, das Existenzminimum konkret zu definieren? Wir haben gestern im Gesprächskreis Verwaltung darüber diskutiert, dass Verwaltungsgerichte inzwischen auf Euro und Cent ausrechnen, welche amtsangemessene Alimentation sich für Beamte mit drei oder mehr Kindern aus Art. 33 Abs. 5 GG ergibt, und zwar ohne gesetzliche Grundlage. Ist es da nicht merkwürdig, dass wir uns bei der vielleicht doch etwas existenzielleren Frage des schieren Existenzminimums so sehr scheuen, sehr konkret zu werden, und insbesondere die Gerichte sich so zurückhalten? Diese Frage knüpft daran an, dass wir das Problem in Art. 1 Abs. 1 GG verankern, denn dann ist natürlich sofort die Befürchtung, dass das über Art. 79 Abs. 3 GG absolut versteinert und der Gestaltungsspielraum des Gesetzgebers, der auch schon von Herrn Fehling angesprochen worden ist, völlig verschwindet. Wäre es nicht logischer, 
Ansatzpunkte hat auch Frau Davy geliefert, den gesundheitlichen Schutz des Existenzminimums in Art. 2 Abs. 2 GG zu verankern und vielleicht auch den angesprochenen sozio-kulturellen Gehalt des Existenzminimums in Art. 2 Abs. 1 GG und daraus abzuleitenden Schutzpflichten für die freie Entfaltung der Persönlichkeit? Dann hätte man nicht gleich an das höchste Rechtsgut angeknüpft, sondern in Verbindung mit dem Sozialstaat an vielleicht auch etwas flexiblere und damit auch für die $\mathrm{Ge}-$ richte handhabbarere Instrumente, ohne dass ich damit den Gestaltungsspielraum des Gesetzgebers ganz aufgeben will.

Peters: Ich möchte an beide Referenten eine Frage aus völkerrechtlicher und verfassungstheoretischer Perspektive stellen. Frau Davy hat in ihrem Referat in den Teilen 3 und 4 von Europäisierung und Internationalisierung der Gleichheit gesprochen. Herr Axer hat in seinem Referat aber in These 3 hervorgehoben, dass der Staat der primäre Adressat der Forderungen nach sozialem Ausgleich sei. Frau Davy hat auch - meiner Ansicht nach völlig zu Recht - betont, dass in Bezug auf das Thema globale Gerechtigkeit und Schutzverantwortung die philosophischen und juristischen Diskurse vollkommen unverbunden nebeneinander stehen. Ich finde, dass dies für die wissenschaftliche Durchdringung des Themas sehr schade und nachteilig ist. Es ist aus juristischer Sicht sicher geboten und von den Tagungsorganisatoren ja auch gewollt, dass wir die vorrechtlichen Voraussetzungen von Verfassungen in den Blick nehmen. Dazu gehört sicher auch der moralische und soziologische Befund. Deshalb meine Frage an beide: Für wie tragfähig halten Sie eine europäische oder gar globale Solidarität als vorrechtliche Voraussetzung für eine europäische oder globale Verfassung? Das Thema wurde ja in dieser Vereinigung schon mal unter der Überschrift „Europäische Identität“ in St. Gallen diskutiert. Können Sie das Problem vielleicht auch zur Frage des demokratischen Prozesses in Beziehung setzen? Herr Axer hat betont, dass, wenn überhaupt, Umverteilungsentscheidungen in einem demokratischen Prozess getroffen werden müssten. Dieser ist bekanntlich auf europäischer Ebene und erst recht auf globaler Ebene defizitär.

Bausback: Im Hinblick auf das Spannungsverhältnis der Freiheit zur Gleichheit haben Sie, Frau Davy, für die internationale Ebene auf das gewandelte Souveränitätsverständnis hingewiesen und weiter versucht, eine „responsibility to protect“ zu begründen. Aber wie weit geht eine solche „responsibility“, eine solche Pflicht, und wem gegenüber gilt sie? Welche konkreten normativen Grundlagen sind auf der internationalen Ebene gegeben? Soziale Menschenrechte, Art. 56 der UN-Charta, soft law - Sie haben einiges genannt. 
Im modernen Völkerrecht ist die Frage nach dem Spannungsverhältnis zwischen Gleichheit und Freiheit vielleicht deshalb besonders interessant, weil zu diesem Spannungsverhältnis das weitere Spannungsfeld zwischen den Staaten als den originären Völkerrechtssubjekten und den Individuen als den Völkerrechtssubjekten sui generis hinzukommt. Wenn Sie in die Völkerrechtsgeschichte schauen, dann ist die Fragestellung einer Solidaritätspflicht ja schon bei Francisco de Vitoria zu finden. In seinem Werk „De Indis recenter inventis relectio prior“ leitet er aus einer solchen Solidaritätspflicht aller Menschen ein ius commercii ab. Aus der Verletzung dieses vermeintlichen Rechts auf Handel und Austausch durch die Eingeborenen rechtfertigt er die spanischen Eroberungen in Amerika. De Vitoria begründet die Solidaritätspflicht noch in erster Linie aus der Gemeinschaft aller menschlichen Individuen. In der weiteren Entwicklung des Völkerrechts tritt dann die bei de Vitoria noch zu findende Bezugnahme auf die Individuen gänzlich zurück. Das Souveränitätsverständnis tritt in den Vordergrund.

Aber was gilt heute, in einem Zeitpunkt, in dem der Einzelne als Träger von unmittelbaren Rechten (Menschenrechten) und Pflichten (z.B. Völkerstrafrecht) Subjekt sui generis des Völkerrechts geworden ist? Auf wen bezöge sich die Solidaritätspflicht, die Sie versucht haben zu begründen? Ist es das Individuum oder ist es der Staat als orginäres Völkerrechtssubjekt? Die Beantwortung dieser Frage hat durchaus praktische Konsequenzen: Sie haben die Weltbank angesprochen. Gibt es eine Berechtigung, die Hilfe für Individuen zu verweigern, weil politische Forderungen nach „good governance“ durch einen Staat nicht erfüllt werden? Wenn Sie die Frage auf ein konkretes Beispiel bezogen haben wollen: Hätte man den Menschen in Simbabwe, die drohten, an Hunger zu sterben, die internationale Hilfe deshalb verweigern dürfen, weil der Diktator Mugabe politische Reformen in dem von ihm regierten Staat verhinderte?

Aber nicht nur die Pflichtenbeziehung ist - wie das Beispiel zeigt heute ungeklärt. Auch stellt sich die Frage, inwieweit eine wirkliche Rechtsgrundlage für eine konkrete Solidaritätspflicht vorhanden ist. Ich bin der Ansicht, dass letztlich die Frage der sozialen Verantwortung, die Sie zurecht anschneiden, auf der internationalen Ebene eben kaum in der rechtlichen, sondern viel mehr in der internationalen politischen Sphäre zu suchen und auch zu lösen ist. Wenn Sie nämlich eine internationale Solidaritätspflicht auf der rechtlichen Ebene ausloten und begründen wollen, kommen Sie in Untiefen, die letztlich nicht zu bewältigen sind. Es würde sich z.B., wenn Sie von einem Recht der in einem Staat lebenden Individuen auf internationale Solidarität ausgehen, auch die Frage stellen, ob es eine humanitäre Intervention im Hinblick auf ex- 
treme soziale Schieflagen geben kann. Konkret: Darf in Simbabwe, Nord-Korea oder einem anderen Staat die internationale Gemeinschaft, die USA oder auch die Europäische Union militärisch intervenieren, wenn die Versorgungslage der Bevölkerung nicht mehr gesichert ist? Soll das universelle Gewaltverbot relativiert werden, wenn soziale Not dies rechtfertigt? Ich denke, gerade die ansonsten anstehende Frage nach einer humanitären Intervention aus sozialer Verantwortung ist ein starkes Argument dafür, eine konkrete Solidaritätspflicht auf der politischen Ebene zu verorten und nicht zu versuchen, eine solche rechtlich im Sinne eines konkreten Anspruchs zu begründen.

Holoubek: Ich darf mich bei allen Diskutantinnen und Diskutanten für die sehr kollegiale Zeitdisziplin bedanken. Wir sind damit hervorragend durch und haben ausreichend Zeit für das Schlusswort der Referentin und des Referenten und beginnen in umgekehrter Reihenfolge.

Axer: Ich möchte mich zuerst herzlich für die Beiträge, Anmerkungen, Anregungen und auch für die vehemente Kritik von Herrn Meyer bedanken. Zugleich bitte ich um Nachsicht, dass ich nicht auf jeden einzelnen Beitrag eingehen kann, werde aber versuchen, soweit wie möglich, zu allen Beiträgen Stellung zu nehmen.

Herr Morlok, Herr Fehling, Herr Groß und Frau Peters thematisierten die Kompetenz des demokratischen Gesetzgebers. Welchen Spielraum besitzt der Gesetzgeber „positiv“ für Maßnahmen zur Herstellung sozialer Gleichheit? Die Schwierigkeiten, den Spielraum „positiv“ zu bestimmen, liegen darin, dass die Sachverhalte, die als Gründe für soziale Ungleichheiten gelten, und das Empfinden sozialer Ungleichheiten im Laufe der Zeit wechseln und changieren können. Inhalt und Gegenstand der Forderungen nach sozialer Gleichheit sind nicht feststehend, sondern wandeln sich; insoweit kann ich auch an zahlreiche Arbeiten von Herrn Zacher anknüpfen. Welche Sachverhalte in einer Gesellschaft zu einem bestimmten Zeitpunkt als nicht mehr hinnehmbare soziale Ungleichheit gelten und Forderungen nach gesetzgeberischem Handeln zum Abbau nach sich ziehen, ist auch zeit- und situationsabhängig und wurde vor zehn Jahren anders gesehen, als es vielleicht in zehn Jahren der Fall sein wird. Dem demokratisch legitimierten Gesetzgeber kommt insoweit die Aufgabe zu, darauf jeweils zu reagieren. Ein Patentrezept, in welcher Weise soziale Ungleichheiten, etwa nur über das Steuerrecht oder nur über das Sozialrecht, zu beheben sind, gibt es nicht.

In Bezug auf hohe Managergehälter stellt sich vor allem die Frage, ob eine Änderung in der Besteuerung oder die Deckelung der Gehälter 
durch Festlegung einer Obergrenze der richtige Weg ist. Der Gesetzgeber kann, wenn er insoweit meint reagieren zu müssen, im Rahmen der grundgesetzlichen Grenzen über das jeweilige Mittel und den konkreten Umfang entscheiden. Nur selten wird allerdings das Grundgesetz einen einzigen bestimmten Weg als den allein verfassungsrechtlich gangbaren zum Abbau sozialer Ungleichheit ausweisen. Bei der Entscheidung über den richtigen Weg ist zudem zu berücksichtigen, dass soziale Gleichheit und Ungleichheit nicht immer Ergebnisse eines statistischen Befundes sind, sondern sie in erheblichem Maße ebenfalls durch die gesellschaftliche Wahrnehmung bestimmt werden. So soll etwa die Armutsquote in den Jahren 2006/2007 leicht zurückgegangen sein, doch führte dies zu keinen spürbaren Veränderungen im Empfinden sozialer Ungleichheit, das nach wie vor sehr stark ist. Der Gesetzgeber hat somit nicht nur auf Statistiken zu reagieren, sondern auch auf die Wahrnehmung sozialer Ungleichheit durch die Gesellschaft. Der ihm dabei zukommende Gestaltungsspielraum ist auch deshalb groß, weil die Herstellung sozialer Gleichheit auf vielfältige Weise erfolgen kann und zahlreiche Probleme aufwirft.

Ein wichtiges Thema für die Herstellung sozialer Gleichheit ist die Bildung, die Herr Hufen und Herr Tomuschat angesprochen haben. Zum Thema Bildung und Chancengleichheit hätte schon allein ein eigenständiges Referat von einer dreiviertel Stunde gehalten werden können. Dass der Bildungsbereich im Vortrag nicht noch öfters angesprochen wurde, lag nicht daran, dass ihm für die Frage der sozialen Gleichheit keine maßgebende Bedeutung zugemessen wurde, sondern daran, dass die Redezeit begrenzt war und das Thema der sozialen Gleichheit sehr weit und vielschichtig ist.

Soweit Herr Tomuschat These neun widerspricht, weil gleiche rechtliche Freiheit in erster Linie soziale Gleichheit erzeuge, hoffe ich in meinem Referat deutlich gemacht zu haben, dass auch meiner Ansicht nach gleiche rechtliche Freiheit eine wichtige Voraussetzung für soziale Gleichheit ist. Allerdings führt rechtliche Gleichheit auch zu sozialer Ungleichheit, wie in These neun formuliert.

Thesen sieben und acht sollen nach der Kritik von Herrn Meyer nicht miteinander vereinbar sein. Dass die Herstellung sozialer Gleichheit eine subsidiäre Staatsaufgabe ist, die im Augenblick auch vom Staat wahrgenommen wird, steht meines Erachtens nicht in Widerspruch zu den bundesstaatlichen Ausführungen in These acht. Zwar lässt der grundgesetzliche Bundesstaat regionale Ungleichheiten $\mathrm{zu}$ und fordert keine umfassende Gleichheit - insoweit glaube ich, dass Übereinstimmung besteht -, doch bleibt die Frage, wie viel soziale Ungleichheit der Bundesstaat verträgt. Dies lässt sich nicht messerscharf bestimmen. Zu- 
dem betrifft soziale Gleichheit, wenn sie in einem ökonomischen Sinne verstanden wird, nur einen Aspekt sozialer Homogenität. Herr Ebsen hat zu Recht darauf hingewiesen, dass soziale Homogenität, wie sie etwa von Hermann Heller verstanden wird, weiter reicht. Im Hinblick auf These 10 - Herr Meyer spricht von großen Worten, die praktisch nicht realisiert werden - ging es mir nicht darum, einen Anspruch auf die notwendigen Voraussetzungen zur Gründung einer Bank oder eines Presseunternehmens zu schaffen, sondern darauf hinzuweisen, dass bestimmte existentielle Voraussetzungen gegeben sein müssen, um Grundrechte ausüben zu können. Soweit Herr Meyer darauf hinweist, dass gleiche Startbedingungen zu sozialer Ungleichheit führen, sehe ich keinen Dissens zu meinem Vortrag, und ich denke mit These 18 auch die mit dem Thema gestellte Frage beantwortet zu haben.

$\mathrm{Ob}$ über die Schutzpflichtenlehre ein grundrechtlicher Anspruch auf bestimmte Maßnahmen zur Herstellung sozialer Gleichheit begründet werden kann, erscheint mir zweifelhaft, wenn die Schutzpflichtenlehre auf den Eingriff privater Dritter beschränkt wird. Das Existenzminimum wird grundsätzlich nicht durch Eingriffe privater Dritter gefährdet, so dass die Gewährleistung des Existenzminimums insoweit kein Fall der Schutzpflichtenlehre ist. Davon unabhängig ist aber nicht zu übersehen - und ich hoffe, dies auch in meinem Referat zum Ausdruck gebracht zu haben -, dass sich gerade aus den Grundrechten Impulse zur Begründung von Ansprüchen ergeben können.

Im Hinblick auf die Frage von Frau Nußberger zur Fundierung und Begründung sozialer Gleichheit aus Art. 3 GG oder dem Sozialstaatsprinzip scheint mir zwischen beiden Grundrechtsbestimmungen kein so großer Gegensatz zu bestehen, wie er zum Teil angenommen wird. Beide Bestimmungen sind vielmehr miteinander verbunden. Selbst wenn der Gleichheitssatz auf rechtliche Gleichheit zielt, muss er auf die soziale Realität blicken und diese aufnehmen. Andererseits kann das Sozialstaatsprinzip zum Zwecke der Herstellung sozialer Gleichheit rechtliche Ungleichheit rechtfertigen. In meinem Vortrag ging es mir darum, anstelle eines scharfen Gegensatzes zwischen Art. 3 GG und dem Sozialstaatsprinzip im Sinne eines „Entweder/Oder“ die Bedeutung beider Vorschriften für die soziale Gleichheit zu betonen.

Herr Welti hat auf die besonderen Gleichheitssätze hingewiesen. Diese konnten angesichts des knappen Zeitbudgets nur kurz angesprochen werden, ohne dass damit die Bedeutung der Regelungen zur Gleichbehandlung der Geschlechter oder der Gleichbehandlung Behinderter für das Thema der sozialen Gleichheit in Abrede gestellt werden sollte.

Warum ist das Existenzminimum nicht gesetzlich definiert? Mit dem SGB II hat der Gesetzgeber zumindest für einen Teil der Leistungen 
eine konkrete, auch die augenblickliche Diskussion bestimmende Summe genannt. Das Problem, das Existenzminimum exakt zu bestimmen, mag auch darin seinen Grund haben, dass sich dessen konkrete Höhe letztlich nur aus einem Vergleich heraus bestimmen lässt und sich zugleich der Bezugspunkt für den Vergleich ständig verändert. Was zudem abstrakt vom Existenzminimum erfasst wird, kann eng definiert und auf das zur Hilfe in Notlagen unbedingt Notwendige beschränkt werden, wie es die Schweizer Bundesverfassung - die auch von Herrn Häberle angesprochen wurde - in Art. 12 GG wohl für die Hilfe in Notlagen vorsieht, oder gerade auch im Hinblick auf ein gewisses Minimum an soziokulturellen Teilhabemöglichkeiten weiter reichen, wie es das Grundgesetz verlangt. Nochmals herzlichen Dank für ihre Beiträge; ich hoffe, dass Meiste beantwortet zu haben.

Davy: Sehr geehrte Damen und Herren! Sie haben mir viele gute Gedanken mit auf den Weg nach Hause gegeben und dafür bin ich dankbar. Ich bin nicht sicher, ob ich im Augenblick angemessen auf Ihre Nachfragen antworten kann. Für drei Fragen will ich gerne eine Antwort versuchen. Erstens: Was war mein Zugang zu ,sozialer Gleichheit“? Zweitens: Warum habe ich mich nicht mit der Frage beschäftigt, ob „soziale Gleichheit" eine Verfassungsvoraussetzung ist? Drittens: Gibt es so etwas wie ,internationale Solidarität“?

Die erste Frage knüpft insbesondere an die Wortmeldung von Herrn Zacher an. Herr Zacher, Sie haben gesagt, dass eine Linie gefunden und gezogen werden müsste zwischen sozialer Ungleichheit, die suspekt ist, und sozialer Ungleichheit, die nicht suspekt ist. Lieber Herr Zacher, ich habe dazu mit großem Gewinn zwei Ihrer Arbeiten zu „Gleichheit“ gelesen. Eine stammt aus dem Jahr 1968 (AöR 93 [1968] 341), die andere aus der Reiner-Schmidt-Festschrift aus dem Jahr 2006. Ich will Ihnen mit Blick auf Ihre '68er-Arbeit selbstverständlich keine Vorhaltungen machen. Es ist aber nicht zu übersehen, dass sich die beiden Arbeiten beträchtlich unterscheiden. In der Arbeit aus dem Jahr 1968 ist soziale Ungleichheit im wesentlichen ,negativ“ besetzt, soziale Ungleichheit erscheint per se als etwas Unerwünschtes. In ihrem Beitrag zur ReinerSchmidt-Festschrift können Sie sozialer Ungleichheit auch etwas Positives abgewinnen. In der Tat ist mir wichtig festzuhalten - darauf habe ich in meinem Bericht Wert gelegt -, dass soziale Ungleichheit nicht in jedem Fall ein Verfassungsproblem ist.

Aus verfassungsrechtlicher Sicht besteht die Aufgabe tatsächlich darin, eine Grenzlinie zu finden, nämlich die Grenzlinie zur verfassungsrechtlich nicht akzeptablen sozialen Ungleichheit. Bei dieser Aufgabe hilft uns allerdings das Sozialstaatsprinzip nicht weiter. Herr Häberle, 
ich habe hierzu vor allem auch Ihren Bericht auf der Jahrestagung der Staatsrechtslehrer 1971 aufmerksam gelesen. Was wir aus dem Sozialstaatsprinzip des Grundgesetzes gewinnen können, hat Herr Axer in seinem Bericht meiner Meinung nach korrekt und an vielen Beispielen sehr einfühlsam beschrieben: Nach dem Gestaltungsauftrag, der im Sozialstaatsprinzip niedergelegt ist, ist es letztlich Aufgabe des einfachen Gesetzgebers, die Grenze zwischen politisch akzeptierter und politisch nicht akzeptierter sozialer Ungleichheit zu ziehen. Freilich, mit diesem Ergebnis wollte ich nicht vor Sie hintreten. Das Ergebnis ist nämlich unter dem Blickwinkel der Rechtsdogmatik wenig befriedigend. Es verweist uns einfach auf die im politischen Raum möglichen Kompromisse. „Dogmatik“ findet hier rasch ein Ende.

Deshalb habe ich mich um einen anderen Zugang zu meinem Thema bemüht. Ich fand Hilfestellung in der Art und Weise, wie die politische Philosophie mit sozialer Ungleichheit umgeht. Das hatte ich im Bericht auch offengelegt: Ich interessiere mich nicht dafür, wie der Gesetzgeber heute mit dem Auftrag umgeht, einen „sozialen Ausgleich“ zu suchen (der Gesetzgeber könnte es ja auch ganz anders machen, und wie er es macht, hängt davon ab, welche Parteien politisches Gewicht besitzen). Aus dogmatischer Sicht befriedigender schien mir die Frage: Kann ich verfassungsrechtlich problematische Fälle von Ungleichheit finden? Solchen Fällen bin ich vor allem im deutschen Teil meines Berichts an verschiedenen Beispielen nachgegangen. Ich habe mich dabei an Fragestellungen orientiert, die die Sozialgerichte konkret beschäftigen - den Gerichten also aktuell unter den Nägeln brennen. Mein Ziel war es, die Rechtsprechung „dogmatisch“ zu kommentieren. Die einzig „harte“ Grenze, die ich aus dogmatischer Perspektive ausfindig machen konnte, ist insoweit das Recht auf Existenzminimum. Damit ist auch der erste Teil der Nachfrage von Frau Nußberger beantwortet. Meine Ausführungen zu den verfassungsrechtlichen Grenzen für soziale Ungleichheit verstehen sich als verfassungsrechtliche, nicht als verfassungspolitische.

Ich wechsle das Thema und komme zu meiner zweiten Frage: Wie halte ich es mit sozialer Gleichheit als Verfassungsvoraussetzung? Darauf bezogen sich mehrere Diskussionsbeiträge. Ich hatte gleich in den ersten Minuten meines Vortrags angekündigt, die Frage nach der Verfassungsvoraussetzung zurückzustellen. Sie können sicher sein, dass dies keine leichtfertige Entscheidung war. Niemand stellt einen Teil der Fragestellung des Vorstands zurück, ohne sich dies gründlich zu überlegen. Ich habe es getan, weil ich mit meinen Überlegungen nicht zu Ende gekommen bin. Aber ich bin damit ein Stück weit gekommen. Und Ihre Nachfragen geben mir Gelegenheit, Ihnen zu zeigen, wie weit ich damit gekommen bin. 
In vielen rechtswissenschaftlichen Arbeiten finden wir den Hinweis, dass zu große ökonomische Differenzen - allgemeiner: zu große soziale Ungleichheiten - für die Demokratie oder für den Bestand des Staates von Übel seien. Ich verweise auf den heute schon mehrfach erwähnten Hermann Heller. Zu nennen wären aber auch Ernst-Wolfgang Böckenförde oder Herbert Krüger. Nun, die Sorge, dass es „zuviel“ soziale Ungleichheit geben könnte, ist gerade in Deutschland außerordentlich plausibel. Zum einen kann man daran erinnern, dass die Sozialreformen des ausgehenden 19. Jahrhunderts zumindest auch von der Sorge getragen waren, die Arbeiterschaft und ihre Parteien könnten das herrschende politische System ernsthaft gefährden. Bismarcks Hauptanliegen war es, die Arbeiter mit dem Reich zu versöhnen. Die Einführung der Sozialversicherung sollte signalisieren, dass der Staat bereit war, soziale Gegensätze nicht zu schroff werden zu lassen. Zum anderen hat die marxistische Kritik rechtsstaatlich-liberale Verfassungen über Jahrzehnte dafür gegeißelt, dass sie lediglich rechtliche Gleichheit, nicht aber soziale Gleichheit garantierten. In der marxistischen Kritik wurde auch nach Einführung der Sozialreformen politische Sprengkraft gesehen. Schließlich gibt es eine Standarderzählung zu den politischen Verhältnissen der Weimarer Republik, die das Mächtigwerden der Nationalsozialisten mit der wirtschaftlichen Not der 20er und 30er Jahre verbindet. Die Behauptung ist, dass nicht so viele Menschen zu den Nazis übergelaufen wären, hätte es nicht eine so weit verbreitete Armut gegeben. All das legt nahe, dass es zwischen Staatsuntergang und sozialer Ungleichheit eine enge Verbindung gibt.

Die Plausibilität der Sorge vor „zuviel“ sozialer Ungleichheit entbindet uns aber nicht von weiterem Nachdenken. Im Gegenteil, das Nachdenken muss gerade an dieser Stelle einsetzen. Denn es muss uns auffallen, dass die Sorge vor „zuviel“ sozialer Ungleichheit in der rechtswissenschaftlichen Forschung regelmäßig nicht empirisch unterfüttert ist. Wenden wir uns an der Stelle hilfesuchend an andere Wissenschaften, finden wir - zugegeben recht abstrakte - Aussagen, die uns zumindest stutzig machen müssen. Soziologische Arbeiten machen uns erstens auf das verbreitete Phänomen der Lethargie aufmerksam. Viele Menschen nehmen die Zustände hin, unter denen sie leben; sie revoltieren dagegen nicht, jedenfalls nicht ohne weiteres. Resignation und das Gefühl, dass Widerstand keinen Zweck hat, bestimmen ihr Leben. Zweitens verweisen uns soziologische Arbeiten auf die stabilisierende Kraft der Hoffnung: Es gibt Staaten, die trotz großer sozialer Ungleichheit stabil sind, weil die Menschen fest daran glauben, ihre Lage eines Tages verbessern zu können und dann zu den Glücklichen zu gehören. Drittens erfahren wir, dass Menschen die Grundlagen von sozialer Un- 
gleichheit, z.B. eine bestimmte Rangordnung, als richtig und angemessen akzeptieren. Davon berichtet v.a. Amartya Sen in verschiedenen seiner Arbeiten. Staatswesen auf der Grundlage eines Kastensystems sind eben nicht in Gefahr, wenn die Menschen Kasten in ihr Wertesystem einbauen. Die Staaten sind durchaus stabil.

Soweit ich sehen kann, gibt es nur eine Person, die der Frage nach dem Verhältnis von sozialer Ungleichheit und der Stabilität von Staaten empirisch nachgegangen ist: Das ist Charles Tilly, der über einen Zeitraum von mehreren hundert Jahren hinweg europäische Staaten untersucht hat, ihren Aufstieg und ihren Untergang. Charles Tilly besitzt außerdem eingehende Kenntnisse über außereuropäische Staaten. Von Charles Tilly lernen wir zweierlei: Es ist danach wohl anzunehmen, dass es vielfach eine Verbindung zwischen (blutigen) Konflikten und Ungerechtigkeit gibt. Menschen können sichtlich vor allem dann mobilisiert und auf die Straße gebracht werden, wenn es gelingt, die herrschenden Zustände als ungerecht zu beschreiben. Allerdings: Was in Konflikten als "Ungerechtigkeit“" thematisiert wird, hat nicht notwendigerweise einen Bezug zu sozialer Ungleichheit. Ich könnte viele Konflikte nacherzählen, die blutig waren und zum Untergang von Staaten geführt haben, die sich aber nicht an sozialer Ungleichheit entzündet haben. Das gilt übrigens gerade auch für die jüngere deutsche Geschichte. Wir lernen von Charles Tilly außerdem - jetzt kann ich an die Wortmeldungen von Herrn Ebsen und Herrn Morlok anschließen -, dass es zwischen demokratischen Systemen und mehr sozialer Gleichheit eine hohe Korrelation gibt: Demokratische Systeme dulden im allgemeinen weniger soziale Ungleichheit als autoritäre Systeme. Folgt man Tilly, kann man daraus aber nicht den Schluss ziehen, dass soziale Gleichheit zumindest für demokratische Systeme eine Verfassungsvoraussetzung ist. Es scheint vielmehr umgekehrt zu sein. Das haben Herr Ebsen und Herr Morlok bereits angedeutet: Demokratische Systeme sind die Voraussetzung für mehr soziale Gleichheit, und zwar aus verschiedenen Gründen. Den wichtigsten Grund will ich kurz erwähnen: Demokratische Systeme sind offen für Lobby-Gruppen; sie lassen es zu, dass Interessengruppen im Parlament vertreten sind. Die Interessengruppen können bei entsprechender Mehrheit - ihre Anliegen durchsetzen, darunter auch das Anliegen, mehr soziale Gleichheit (für sie) herzustellen. Herr Zacher hat die Wechselwirkungen zwischen Sozialstaat und Demokratie oft beschrieben.

Führt man alle diese Arbeiten zusammen, würde ich vorsichtig sagen: Nein, mehr soziale Gleichheit ist nicht zwingend eine Verfassungsvoraussetzung. Es gibt Staaten, die ungeachtet großer sozialer Ungleichheit gut funktionieren und in einem globalen Kontext sogar „demokra- 
tisch“ genannt werden können. Botswana ist dafür ein auffälliges Beispiel. In Botswana liegt die Lebenserwartung nach den Berichten des U.S. Department of State bei 34 Jahren. Nach den Veröffentlichungen der Weltbank gehört Botswana zu den fünf Staaten mit dem höchsten Gini-Koeffizienten (er liegt bei 60,5). Und dennoch: Nach einem auf globale Verhältnisse zugeschnittenen niedrigschwelligen Demokratiebegriff ist Botswana eine stabile Demokratie, und das seit vielen Jahrzehnten.

Damit bin ich bei der dritten und letzten Frage, auf die ich eingehen will: Gibt es so etwas wie internationale Solidarität? Ich will hier zunächst an den Bericht von Ewald Wiederin bei der Jahrestagung der Vereinigung im Jahr 2004 in Jena anknüpfen. Lieber Ewald, ich tue das, weil Du einen Gedanken, den man auch in anderen Arbeiten finden kann, sehr schön zugespitzt hast: Nach einer verbreiteten Überzeugung findet man Solidarität zuerst in der Familie, dann in der Nation bzw. im Staat, und das - so hast Du gemeint - vor allem dann, wenn der Staat homogen ist. Ich akzeptiere das für einen Moment und schließe daraus für ,internationale“ Solidarität: Internationale Solidarität ist ein Unding, entweder, weil die nötige Homogenität fehlt oder aus anderen nicht offengelegten Gründen.

Ich bezweifle die Richtigkeit dieser These. Es lässt sich nämlich ziemlich präzise feststellen, wann Solidarität mit den „Armen“ der Nation historisch entstanden ist. Solidarität mit den „Armen“ der Nation entstand, als sich eine völkergewohnheitsrechtliche Norm entwickelte, die es den europäischen Staaten untersagte, verarmte Staatsangehörige aus dem Lande zu schaffen. Diese völkergewohnheitsrechtliche Norm entstand in der Mitte des 19. Jahrhunderts. Bis dahin war es den Staaten erlaubt, mittellose Menschen - selbst wenn sie eigene Angehörige waren aus dem Staatsgebiet zu vertreiben. Europäische Staaten haben davon auch reichlich Gebrauch gemacht. Noch zu Beginn des 19. Jahrhunderts gab es also keine Solidarität innerhalb der Nation. Diese Solidarität hat sich unter bestimmten politischen Bedingungen entwickelt. Man kann sogar sagen, dass die europäischen Staaten die Verantwortung für „ihre“ Armen nur zähneknirschend akzeptiert haben (die Alternative schien ein noch größeres Übel zu sein). Auf diese Weise entstanden Fürsorgesysteme, die von bestimmten Menschen - den Staatsangehörigen - in Anspruch genommen werden konnten, ohne dass diese fürchten mussten, ihr Aufenthaltsrecht zu verlieren. Die staatliche Fürsorge des ausgehenden 19. Jahrhunderts war im übrigen alles andere als Ausdruck einer besonderen Brüderlichkeit. Wer die Fürsorge in Anspruch nahm, verlor die politischen Rechte. Arme waren keinesfalls Gleiche.

Aber wie dem auch unter dem Aspekt der Brüderlichkeit sei, wichtig ist: Nationale Solidarität entstand in einer spezifischen historischen Si- 
tuation. Sie ist ein politisches Konstrukt. Daran schließt sich ein weiteres Argument: Wenn nationale Solidarität ein Konstrukt ist, warum soll es nicht möglich sein, Solidarität zu konstruieren, die über die nationalen Grenzen hinaus geht? In der Tat können wir für eine derart erweiterte Solidarität eine Reihe von Anhaltspunkten finden. Wir finden Anhaltspunkte für grenzüberschreitende Solidarität in Europa. Der europäische Sozialfonds ist wahrscheinlich ein herausragendes Beispiel für europäische Solidarität. Aber es gibt auch andere, v.a. im Kontext der Freizügigkeit. Einige habe ich in meinem Bericht angesprochen. Und wenn wir bereit sind, danach zu suchen, dann finden wir darüber hinaus Anhaltspunkte für eine internationale Solidarität. Auf globaler Ebene sind Strukturen entstanden, wie die Vereinten Nationen, NGO's oder weltweit operierende Unternehmen, die Solidarität zum Gegenstand ihres Handelns machen. Es gibt heute außerdem so etwas wie eine globale Öffentlichkeit, die Solidarität zu einem politischen Thema macht. Wer das möchte, kann über das internet ohne weiteres an dieser globalen Öffentlichkeit teilhaben. Die großen Konferenzen auf dem Gebiet der Entwicklungspolitik oder der Welthandelspolitik werden heute stets weltöffentlich begleitet: Sie finden websites, auf denen Sie sich nicht nur über die Standpunkte der einzelnen Staaten, sondern auch über die Beiträge von internationalen Organisationen und NGO's informieren können. Wenn Sie wollen, können Sie sich auch aktiv an den politischen Diskussionen beteiligen. Der Nationalstaat ist also keineswegs der einzige und möglicherweise bald auch nicht mehr der einzig selbstverständliche Bezugspunkt unserer Solidarität.

Ich habe Ihre Aufmerksamkeit über Gebühr beansprucht. Ich danke Ihnen. 